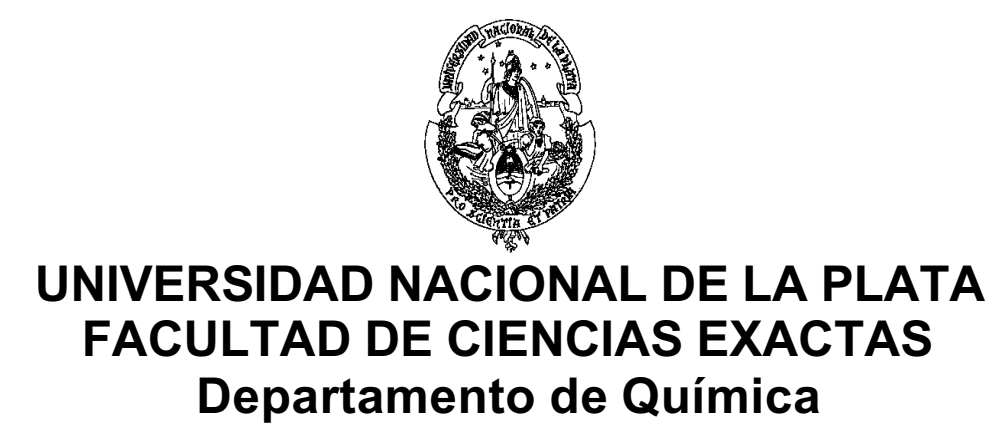

\title{
SÍNTESIS Y ESTUDIOS FOTOQUÍMICOS Y FOTOFÍSICOS DE COMPLEJOS TRICARBONÍLICOS DE Re'
}

\author{
GUSTAVO TEODOSIO RUIZ
}

Lugar de trabajo: Sección Cinética y Fotoquímica en Solución, Instituto de Investigaciones Fisicoquímicas Teóricas y Aplicadas, INIFTA.

Director de Tesis: Dr. Mario R. Féliz Co-Director de Tesis: Dr. Guillermo Ferraudi

Trabajo de Tesis presentado para optar al título de

Doctor de la Facultad de Ciencias Exactas 
A mi esposa V iviana

A mamá y papá

A toda mi familia 
Deseo expresar mi agradecimiento a todas las personas que de una manera $\mathrm{u}$ otra contribuyeron para que este trabajo de Tesis pudiera ser llevado a cabo, especialmente:

A vos Mario, no solo por brindarme toda tu experiencia profesional, dirigiendo este trabajo de Tesis y alentándome constantemente, sino también por estar ahí cada vez que te necesité, ya sea para medir, diseñar experimentos, analizar los datos ó sentarnos a leer juntos algo que no entendía. También, por darme tu confianza y libertad para desarrollar mi trabajo. Además, por supuesto, por las incontables e interesantes charlas y discusiones, almuerzo ó café de por medio.

A usted Alberto, por darme la posibilidad de iniciarme en la investigación científica, convirtiéndose en el principal "culpable" de mi actividad actual y, espero, futura. Además, por todo el apoyo que siempre me ha dispensado.

A vos Guillermo, por brindarme tu enorme conocimiento y apoyo constante, co-dirigiendo este trabajo de Tesis y ayudándome en la interpretación de los resultados. Además, por haberme recibido en tu laboratorio del Radiation Laboratory, en la Universidad de Notre Dame, USA, donde tuve la oportunidad de realizar experimentos de láser flash fotólisis y ganar experiencia profesional.

A vos Vivi, simplemente por todo, ya que sin tu amor, comprensión, aliento, consejos, alegría, apoyo, confianza, sensibilidad y mucha, mucha, paciencia, no me hubiera sido posible llevar a cabo este trabajo doctoral.

A vos Paula, por ayudarme en el trabajo experimental y por tu incondicional apoyo, aliento, confianza cariño, compañerismo y permanente predisposición para ayudarme siempre, pero, fundamentalmente, por ser mi amiga. 
A vos Ezequiel, por haber colaborado conmigo en la realización de casi todas las medidas experimentales y análisis de datos, y por brindarme tu ayuda y compañerismo a lo largo de más de 11 años de trabajo en conjunto. También, por asesorarme permanentemente en el uso del láser flash fotólisis acá y en Estados Unidos. Además, por los momentos compartidos en los viajes que nos tocó hacer juntos.

Al Consejo Nacional de Investigaciones Científicas y Técnicas (CONICET), por la beca de Formación de Posgrado otorgada para realizar el Doctorado.

A la Fundación Antorchas, por la beca otorgada para realizar una pasantía laboral en el Radiation Laboratory, University of Notre Dame, Indiana, USA.

A las autoridades de la Facultad de Ciencias Exactas y, en particular, a las del Instituto de Investigaciones Fisicoquímicas Teóricas y Aplicadas (INIFTA), por haber accedido a que realizara mis actividades en esta institución.

A todos mis compañeros de trabajo del primer piso del INIFTA, Mario, Paula, Ezequiel, Alberto, Laurita, Andrés, Seba, Caro, Franco, Jorge, Daniel, Mónica, Janina, Carlos, Adela, M. Eugenia, Norma, Juanita, Paula B., junto con los más nuevos, Paula C., Gabriela, Laura, por compartir todas las vicisitudes de nuestra actividad, por todo el afecto y compañerismo recibido y por todos los gratos momentos compartidos.

Al Dr. Reymaldo Lezna, por poner a disposición todo su equipamiento y conocimiento en las medidas electroquímicas llevadas a cabo en su laboratorio y en la interpretación de los resultados. 
A Juan Guerrero, de la Universidad de Santiago de Chile, por ayudarme en la interpretación de los espectros de ${ }^{1} \mathrm{H}-\mathrm{RMN}$; a Romina Gómez, por haber colaborado conmigo en la síntesis de la fendiona y a Eliana Juliarena por su ayuda desinteresada en la confección de algunos gráficos del trabajo.

A vos Cachito, por estar siempre cerca a pesar de la distancia.

Al Dr. Sicre y al Dr. Castellano, por sus interesantes charlas de café y por compartir la misma pasión gimnasista y a vos, Tori, por todas las alegres discusiones futboleras, a pesar de que nunca compartirás mi sentimiento tripero.

A todo el personal científico, técnico y administrativo del INIFTA, por su cercanía y asistencia permanente.

A toda mi familia, en especial a los más chiquitos y a los que fueron y siguen siendo chicos para mí, por darme la fuerza y vitalidad necesarias para seguir adelante y superar algunos momentos difíciles que tuvimos que afrontar.

A mis amigos de toda la vida, por darme la confianza y seguridad de que siempre van a estar conmigo. 


\section{PARTE I: PARTE EXPERIMENTAL}

Introducción

CAPITULO 1: Descripción de las técnicas utilizadas

Procesos fotofísicos y fotoquímicos en complejos de metales de transición 8

Descripción de los orbitales moleculares $\quad 8$

Fotofísica 11

i. Decaimiento radiativo 14

ii. Efectos del solvente $\quad 15$

iii. Emisión $^{3}\left(\pi^{*} \rightarrow \pi\right) \quad 16$

iv. $\quad$ Quenching bimolecular $\quad 17$

$\begin{array}{ll}\text { Fotoquímica } & 18\end{array}$

Monitoreo de los procesos del estado excitado 19

Método de flash fotólisis $\quad 21$

i. $\quad$ Descripción general del método y detalles experimentales 21

ii. $\quad$ Componentes del equipo de láser flash fotólisis 25

iii. $\quad$ Procesamiento de los datos $\quad 27$

Experimentos de fotólisis estacionaria $\quad 30$

Luminiscencia y espectrofluorometría

i. $\quad$ Emisión en estado estacionario: espectros de emisión, espectros de excitación y rendimientos cuánticos de emisión 32

ii. $\quad$ Estimación de las energías de punto 0-0 $\left(E_{00}\right)$

iii. $\quad$ Emisión resuelta en el tiempo $\quad 40$

$\begin{array}{ll}\text { Electroquímica } & 42\end{array}$

i. $\quad$ Voltametría cíclica $\quad 42$

ii. $\quad$ Espectroelectroquímica 43

Bibliografía consultada para la Parte I del presente trabajo de tesis 48 
PARTE II: SINTESIS, ESTUDIOS FOTOQUIMICOS, FOTOFISICOS Y

FOTOELECTROQUIMICOS DE UN COMPLEJO DE Re ${ }^{\mathrm{I}}$ DEL TIPO

fac-[L-COO-Re ${ }^{I}(C O)_{3}(2,2$ 'bipiridina $\left.)\right]$

Introducción

CAPITULO 2: Preparación y caracterización de

fac-[Antraquinona-2-COO-Re $\boldsymbol{I}^{I}(\mathrm{CO})_{3}\left(2,2^{\prime}\right.$ 'bipiridina)]

Detalles Experimentales $\quad 54$

Materiales $\quad 54$

Síntesis y caracterización de $\left.f a c-\left[A n t r a q u i n o n a-2-C O O-R e^{I}(C O)\right)_{3}(2,2 ' b i p i)\right] \quad 54$

i. $\quad f a c-\left[\operatorname{BrRe}^{I}(\mathrm{CO})_{3}\left(2,2^{\prime}\right.\right.$ bipi $\left.)\right]$

ii. Antraquinona-2-COOAg 55

iii. $\quad$ fac-[Antraquinona-2-COO-Re ${ }^{I}(\mathrm{CO})_{3}\left(2,2^{\prime}\right.$ 'bipi)] 56

Técnicas y equipamiento utilizados 56

$\begin{array}{ll}\text { Resultados } & 57\end{array}$

$\begin{array}{ll}\text { Síntesis } & 57\end{array}$

Espectros de absorción infraroja $\quad 61$

Espectros ${ }^{1} \mathrm{H}-\mathrm{RMN}$

Espectros de absorción UV-visible $\quad 66$

$\begin{array}{ll}\text { Medidas electroquímicas } & 70\end{array}$

$\begin{array}{ll}\text { Conclusiones } & 74\end{array}$

CAPITULO 3: Propiedades fotoquímicas y fotofísicas de

fac-[Antraquinona-2-COO-Re $\boldsymbol{I}^{I}(\mathrm{CO})_{3}(2,2$ 'bipiridina)]

Resultados experimentales y Discusión 76

$\begin{array}{ll}\text { Materiales } & 76\end{array}$

$\begin{array}{ll}\text { Equipos y métodos } & 76\end{array}$

Espectroscopía de absorción y emisión UV-visible 78

Medidas electroquímicas $\quad 82$

i. $\quad$ Potenciales de media onda 82 
ii. $\quad$ Espectroelectroquímica 83

Espectroscopía resuelta en el tiempo $\quad 87$

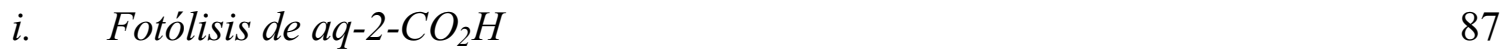

ii. Fotólisis de aq-2-CO $-\mathrm{Re}^{I}(\mathrm{CO})_{3}\left(2,2^{\prime}\right.$ bipi) 94

$\begin{array}{ll}\text { Conclusiones } & 104\end{array}$

CAPITULO 4: Espectroelectroquímica UV-visible / IR de fac-[Antraquinona-2-COO-R $e^{I}(C O)_{3}\left(2,2^{\prime}\right.$ bipiridina)]

Resultados experimentales y Discusión 109

$\begin{array}{ll}\text { Materiales } & 109\end{array}$

$\begin{array}{ll}\text { Equipos y métodos } & 109\end{array}$

Medidas electroquímicas $\quad 111$

i. $\quad$ Voltametría cíclica de alterna 111

ii. $\quad$ Espectros UV-visible de las especies reducidas 112

iii. $\quad$ Espectros IR in-situ 115

$\begin{array}{ll}\text { Conclusiones } & 121\end{array}$

Bibliografía consultada para la Parte II del presente trabajo de tesis 122

PARTE III: SINTESIS, ESTUDIOS FOTOQUIMICOS Y FOTOFISICOS DEL LIGANDO DPPZ (dipirido[3,2-a:2',3'-clfenacina) Y DE UN COMPLEJO DE Re ${ }^{\mathrm{I}}$ DEL TIPO [fac-(dppz) $\operatorname{Re}^{I}(\mathrm{CO})_{3}\left(4,4^{\prime}\right.$ 'bipiridina)] $\left(\mathrm{O}_{3} \mathrm{SCF}_{3}\right)$

Introducción

CAPITULO 5: Preparación y caracterización del ligando dppz (dipirido[3,2-a:2',3'-c] fenacina) y del [fac-(dppz) $\operatorname{Re}^{I}(\mathrm{CO})_{3}\left(4,4^{\prime}\right.$ bipiridina) $]\left(\mathrm{O}_{3} \mathrm{SCF}_{3}\right)$

Detalles Experimentales

Síntesis y caracterización de $\left[\right.$ fac-(dppz) $\operatorname{Re}^{\mathrm{I}}(\mathrm{CO})_{3}(4,4$ 'bipi $\left.)\right]\left(\mathrm{O}_{3} \mathrm{SCF}_{3}\right)$

ii. fac-(1-10 fenantrolina 5,6 diona) $\operatorname{Re}^{I}(\mathrm{CO})_{3} \mathrm{Cl}$ 
iii. $\quad f a c-(d p p z) \operatorname{Re}^{I}(C O)_{3} C l$

iv. $\quad\left[\mathrm{fac}-(\mathrm{dppz}) \mathrm{Re}^{I}(\mathrm{CO})_{3}\left(\mathrm{CH}_{3} \mathrm{CN}\right)\right]\left(\mathrm{O}_{3} \mathrm{SCF}_{3}\right)$

v. $\left[\mathrm{fac}-(\mathrm{dppz}) \operatorname{Re}^{I}(\mathrm{CO})_{3}\left(4,4^{\prime}\right.\right.$ bipi $\left.)\right]\left(\mathrm{O}_{3} \mathrm{SCF}_{3}\right)$

Técnicas y equipamiento utilizados

Resultados

Síntesis

Espectros de absorción infraroja

Espectros ${ }^{1} \mathrm{H}-\mathrm{RMN}$

Espectros de absorción UV-visible

Medidas electroquímicas

Conclusiones

CAPITULO 6: Estudios fotoquímicos y fotofísicos realizados sobre el ligando dppz

Resultados experimentales y Discusión

Materiales

Equipos y métodos

Espectros de absorción y de emisión UV-visible

Experimentos de láser flash fotólisis

Experimentos de fotólisis estacionaria

Conclusiones

CAPITULO 7: Propiedades fotoquímicas y fotofísicas del

[fac-(dppz) $\operatorname{Re}^{I}(\mathrm{CO})_{3}\left(4,4\right.$ 'bipiridina)] $\left(\mathrm{O}_{3} \mathrm{SCF}_{3}\right)$

Resultados experimentales y Discusión

Materiales

Equipos y métodos

Espectros de absorción y de emisión UV-visible 170

$\begin{array}{ll}\text { Estudio del quenching de la emisión por agua } & 175\end{array}$

$\begin{array}{ll}\text { Experimentos de láser flash fotólisis } & 179\end{array}$

i. $\quad$ Estudio de las especies transitorias generadas en distintos solventes 
ii. $\quad$ Quenching bimolecular del estado excitado 184

Experimentos de fotólisis estacionaria $\quad 189$

$\begin{array}{ll}\text { Conclusiones } & 191\end{array}$

CAPITULO 8: Estudio de interacción del [fac-(dppz) $\operatorname{Re}^{I}(\mathrm{CO})_{3}(4,4$ 'bipiridina $\left.)\right]\left(\mathrm{O}_{3} \mathrm{SCF}_{3}\right)$ con polinucleótidos sintéticos

Resultados experimentales y Discusión 197

Materiales y equipos utilizados

Estudios de interacción realizados entre el $(\mathrm{dppz}) \mathrm{Re}^{\mathrm{I}}(\mathrm{CO})_{3}\left(4,4^{\prime} \mathrm{bipi}\right)^{+}$ y el Poly[dAdT $]_{2}$

i. $\quad$ Espectros de absorción UV-visible

ii. $\quad$ Espectros de emisión $\quad 202$

iii. $\quad$ Determinación de la constante de asociación ó constante de binding $K_{b} \quad 205$

Estudios de interacción realizados entre el (dppz) $\operatorname{Re}^{\mathrm{I}}(\mathrm{CO})_{3}\left(4,4^{\prime} b i p i\right)^{+}$

y el Poly[dGdC $]_{2}$

i. $\quad$ Espectros de absorción UV-visible 210

ii. $\quad$ Espectros de emisión 211

$\begin{array}{ll}\text { Conclusiones } & 214\end{array}$

Bibliografía consultada para la Parte III del presente trabajo de tesis 218 
INTRODUCCIÓN GENERAL Y OBJETIVOS 
Las propiedades fotoquímicas y fotofísicas de complejos tricarbonílicos de $\operatorname{Re}^{\mathrm{I}}$ del tipo $\mathrm{XRe}^{\mathrm{I}}(\mathrm{CO})_{3} \mathrm{~L}_{2}$, donde $\mathrm{X}=$ haluro, acina ó fosfina; $\mathrm{L}=$ acina monodentada ó $\mathrm{L}_{2}=$ acina bidentada han sido objeto de numerosas investigaciones desde mediados de la década del ' 70 , cuando se pudieron describir por primera vez las propiedades de los estados excitados en algunos compuestos de esta familia [1-31]. Las investigaciones en este campo, continúa siendo de gran interés por la potencial aplicación de estos compuestos: en fotocatálisis [5,8-9,1623,25,29], como sensores luminiscentes, en la derivatización de polímeros fotoreactivos, como sensibilizadores semiconductores, en sondas específicas para biomoléculas, como inductores de fotoclivaje de ADN, etc. [30,32,33].

El objetivo de este trabajo de Tesis es aportar información sobre las propiedades fotoquímicas $\mathrm{y}$ fotofísicas de nuevos complejos tricarbonílicos de $\mathrm{Re}^{\mathrm{I}}$ que contienen ligandos piridínicos bidentados.

En la parte I se describen los estados excitados puestos en juego luego de la absorción de luz apropiada, en complejos de metales de transición $d^{6}$ y sus implicancias en los procesos fotoquímicos y fotofísicos. Además, se describen las técnicas y equipamiento utilizados para el estudio y monitoreo de esos estados excitados (capítulo 1)

En la parte II se presenta la síntesis y caracterización del complejo fac-[Antraquinona-2COO-Re ${ }^{\mathrm{I}}(\mathrm{CO})_{3}\left(2,2^{\prime}\right.$ bipiridina)] (capítulo 2) y los estudios fotoquímicos, fotofísicos y de espectroelectroquímica realizados (capítulos 3 y 4).

Por último, en la parte III, se describe el método de obtención y caracterización del ligando orgánico dipirido[3,2-a:2',3'-c]fenacina (dppz) y del complejo [fac$(\mathrm{dppz}) \operatorname{Re}^{\mathrm{I}}(\mathrm{CO})_{3}\left(4,4^{\prime}\right.$ bipiridina $\left.)\right]\left(\mathrm{O}_{3} \mathrm{SCF}_{3}\right)$ (capítulo 5). En esta ocasión se realizó un estudio profundo de las propiedades espectroscópicas del ligando libre (capítulo 6) y posteriormente, un análisis detallado de los estados excitados del complejo de $\operatorname{Re}^{\mathrm{I}}$ en estudio (capítulo 7). Finalmente, se describen algunos aspectos de la interacción de este último complejo con 
polinucleótidos sintéticos y su potencial aplicación como sonda luminiscente de ADN en sistemas biológicos.

\section{Referencias}

[1] Wrighton, M.S.; Morse, D.L. J. Am. Chem. Soc. 1974, 96, 998.

[2] Wrighton, M.S.; Morse, D.L; Pdungsap, L. J. Am. Chem. Soc. 1975, 97, 2073.

[3] Luong, J.C.; Faltynak, H.; Wrighton, M.S. J. Am. Chem. Soc. 1979, 101, 1597.

[4] Giordano, P.J.; Fredericks, S.M.; Wrighton, M.S.; Morse, D.L. J. Am. Chem. Soc. 1978, $100,2257$.

[5] Luong, J.C.; Nadjo, L.; Wrighton, M.S. J. Am. Chem. Soc. 1978, 100, 5790.

[6] Giordano; P.J.; Wrighton, M.S. J. Am. Chem. Soc. 1979, 101, 2888.

[7] Fredericks, S.M.; Luong, J.C.; Wrighton, M.S. J. Am. Chem. Soc. 1979, 101, 7415.

[8] Summers, D.P.; Luong, J.C.; Wrighton, M.S. J. Am. Chem. Soc. 1981, 103, 5238.

[9] Shu, C.; Wrighton, M.S. Inorg. Chem. 1988, 27, 4326.

[10] Sacksteder, L.; Zipp, A.P.; Brown, E.A.; Streich, J.; Demas, J.N.; Degraff, B.A. Inorg. Chem. 1990, 29, 4335.

[11] Perkins, T.A.; Humer, W.; Netzel, T.L.; Schanze, K.S. J. Phys. Chem. 1990, 94, 2229.

[12] Shaw, J.R.; Schmehl, R.H.; J. Am. Chem. Soc. 1991, 113, 389.

[13] Glezen, M.M.; Lees, A.J. J. Am. Chem. Commun. 1987, 1752.

[14] Glezen, M.M.; Lees, A.J. J. Am. Chem. Soc. 1988, 110, 3892.

[15] Glezen, M.M.; Lees, A.J. J. Am. Chem. Soc. 1988, 110, 6243.

[16] Hawecker, J.; Lehn, J.M.; Ziessel, R.; J. Chem. Soc., Chem. Commun. 1984, 328.

[17] Kutal, C; Weber, M.A.; Ferraudi, G.; Geiger, D. Organometallics 1985, 4, 2161.

[18] Kutal, C.; Corbin, A.J.; Ferraudi, G.; Geiger, D. Organometallics 1987, 6, 553. 
[19] Féliz, M.R.; Ferraudi, G. Chem. Phys. Lett. 1991, 181, 201.

[20] Caspar, J.V.; Meyer, T.J. J. Phys. Chem. 1983, 87, 952.

[21] Sullivan, B.P.; Meyer, T.J. J. Chem. Soc., Chem. Commun. 1984, 1244.

[22] Caspar, J.V.; Sullivan, B.P.; Meyer, T.J. Inorg. Chem. 1984, 23, 2104.

[23] Westmoreland, T.D.; Schanze, K.S.; Neveux, Jr., P.E.; Danielson, E.; Sullivan, B.P.; Chen, P.; Meyer, T.J. Inorg. Chem. 1985, 24, 2596.

[24] G. Ferraudi, Neyde Murakami Iha, G. Ruiz, E. Wolcan and M.R.Féliz, Inorg. Chim. Acta, 221, 1994, 161-164

[25] G.Ruiz, E.Wolcan, A.L.Capparelli and M.R.Féliz, J. Photochem. Photobiol. A: Chem. $89,1995,61-66$

[26] G. Ruiz, E. Wolcan and M. R. Féliz, J. Photochem. Photobiol. A: Chem. 101, 1996, $119-125$

[27] G. Ruiz, F. Rodriguez Nieto, E. Wolcan and M. R. Féliz, J. Photochem. Photobiol. A: Chem. 107, 1997, 47-54

[28] M. R. Féliz, F. Rodriguez Nieto, G. Ruiz and E. Wolcan, J. Photochem. Photobiol. A: Chem. 117, 1998, 185-192.

[29] Kalyanasundaram K. and Grätzel M. “Photosensitization and Photocatalysis Using Inorganic and Organometallic Compounds” Kluwer Academic Publishers, Dordrecht, 1993.

[30] Stufkens D.J. and Vlèek Jr A. Coord. Chem. Rev. 177 (1998) 127-179.

[31] L.S. Forster, Coord. Chem. Rev., 227, 2002, 59-92

[32] Autores varios, “Applications of Inorganic Photochemistry”, J. Chem. Educ. 1997, Vol 74, Nro.6, 633-702.

[33] K.E. Ekkila, D.T. Odom and J.K. Barton, Chem. Rev. 1999, 99, 2777-2795. 
PARTE I

PARTE EXPERIMENTAL 


\section{Introducción}

En este capítulo se describen brevemente el fundamento de los métodos y técnicas utilizadas en este trabajo de tesis para la observación de los procesos que involucran a los estados excitados fotogenerados en complejos polipiridílicos de metales de transición $\mathrm{d}^{6}$.

La fotoquímica y fotofísica de los complejos de esta familia de compuestos, a la cual pertenecen los complejos tricarbonílicos de $\mathrm{Re}^{\mathrm{I}}$, es análoga a la que presenta el complejo $\operatorname{Ru}\left(2,2^{\prime} \text { 'bipi }\right)_{3}{ }^{+2}$ (2,2'bipi $=2,2^{\prime}$ 'bipiridina $)$. Siendo este compuesto muy estudiado, puede elegirse para describir los probables estados excitados presentes luego de la excitación con luz apropiada. Por lo tanto y, previo a la descripción técnica, introduciremos algunos conceptos usando este complejo como "modelo".

Posteriormente, se describen las técnicas para el estudio de los estados excitados, fundamentalmente, el método de láser flash fotólisis; técnicas para el estudio de luminiscencia de estado estacionario y resuelta en el tiempo; técnicas electroquímicas de caracterización de especies reducidas (espectroelectroquímica) y otras utilizadas en este trabajo.

Otros detalles experimentales se mencionan en los capítulos correspondientes al caso en particular considerado. 
CAPITULO 1

DESCRIPCIÓN DE LAS TÉCNICAS UTILIZADAS

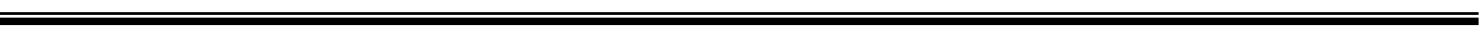




\section{Procesos fotofísicos y fotoquímicos en complejos de metales de transición}

\section{Descripción de los orbitales moleculares}

Los complejos de Re en estado de oxidación +1 presentan una configuración electrónica en la cual los orbitales $5 d$ poseen 6 electrones. En general, los complejos de metales de transición $d^{6}$ que poseen en su esfera de coordinación ligandos polipiridínicos, presentan estados excitados que se asemejan fuertemente a los observados para el $\mathrm{Ru}\left(2,2^{\prime} \text { bipi }\right)_{3}{ }^{+2}$. Este, y otros complejos relacionados, ha sido ampliamente estudiado y se presentará aquí como complejo "modelo" para entender, de manera simplificada, la dinámica de los estados excitados puestos en juego luego de la excitación electrónica. Los rasgos particulares de complejos tricarbonílicos de $\mathrm{Re}^{\mathrm{I}}$ serán comentados convenientemente.

En la figura 1 se ilustra el diagrama de orbitales moleculares resultante de la combinación de los orbitales $4 \mathrm{~d}$ del $\mathrm{Ru}^{\mathrm{II}} \mathrm{y}$ orbitales $n, \pi \mathrm{y} \pi^{*}$ de la 2,2'bipi. En un entorno pseudo-octahédrico, los cinco orbitales $4 \mathrm{~d}$ se dividen en dos grupos, como producto de su diferente simetría y energía de repulsión, generada luego de la coordinación de cada ligando y su ubicación en el espacio octahédrico: dos orbitales $e_{\mathrm{g}} \mathrm{y}$ tres $\mathrm{t}_{2 \mathrm{~g}}$. Los primeros son de mayor energía y por lo tanto se los ha denominado también como orbitales $\mathrm{d} \sigma^{*}$-metal, mientras que los segundos, que disminuyen su energía con la coordinación, se denominan orbitales d $\pi$-metal. Los 6 electrones 4d están apareados y se ubican en estos tres orbitales degenerados, de naturaleza no enlazante (complejos de bajo spin). La magnitud, en energía, de la separación entre los orbitales e $\mathrm{g}_{\mathrm{g}}$, $\Delta_{0}$ o $10 \mathrm{Dq}$, depende de algunos parámetros que incluyen el radio iónico y carga del metal y la naturaleza química de los ligandos. Los ligandos polipiridínicos tienen orbitales $n$ no

enlazantes y orbitales $\pi$ ocupados ó llenos, y orbitales $\pi^{*}$ anti-ligantes vacíos de baja energía. Los pares de electrones no enlazantes de los átomos de nitrógeno (u orbitales $n$ llenos) 
participan en la formación de enlaces metal-ligando, estabilizándose y disminuyendo sustancialmente su energía. En función de este diagrama simplificado se pueden identificar las diferentes transiciones electrónicas posibles en esta familia de compuestos luego de la absorción de luz apropiada.

La aparición de una intensa absorción en la región visible es un rasgo característico de estos complejos y se corresponde con una transferencia de carga del metal hacia el ligando, TCML. Estas transiciones ocurren cuando un electrón alojado en un orbital localizado sobre el metal es promovido hacia un orbital localizado sobre el ligando, $\mathrm{t}_{2 \mathrm{~g}} \rightarrow \pi_{\mathrm{L}}{ }^{*}$ en la figura 1 , presentando típicamente, valores del coeficiente de extinción, $\varepsilon \geq 10^{4} \mathrm{M}^{-1} \mathrm{~cm}^{-1}$, ya que están permitidas por las reglas de Laporte y usualmente por las del spin. Además se caracterizan por su tendencia a producir separación de cargas, las cuales pueden llevar a reacciones redox. Las transiciones en las cuales la carga electrónica es transferida esencialmente desde los ligandos hacia el metal coordinante, pertenecen a otro tipo de transferencia de carga denominada transferencia de carga de ligando a metal, TCLM, $\pi_{\mathrm{L}} \rightarrow \mathrm{e}_{\mathrm{g}}$ en la figura 1. Que se verifique una u otra transición, estará determinada por la capacidad donora ó aceptora de electrones de los ligandos y la capacidad de oxidarse ó reducirse del metal central. Por último, transferencia de carga desde el metal hacia el solvente puede, en ocasiones, también observarse. La posición de la banda de absorción depende de la facilidad que tenga el solvente para reducirse y el centro metálico para oxidarse. Estas transiciones donde la carga se mueve hacia el solvente se denominan transferencia de carga al solvente, TCS y pueden llegar a tener valores de $\varepsilon$ de $10^{3} \mathrm{M}^{-1} \mathrm{~cm}^{-1}$.

Además de las transferencias de carga, pueden presentarse transiciones en donde solamente están involucrados orbitales de los ligandos y se denominan transiciones $\pi \rightarrow \pi^{*}$ centradas en el ligando ó transiciones intraligando, IL, $\pi_{\mathrm{L}} \rightarrow \pi_{\mathrm{L}}{ }^{*}$ en la figura 1. Estas transiciones son relativamente poco afectadas por los centros metálicos (se corren levemente hacia el rojo con 
respecto a la correspondiente transición $\pi \rightarrow \pi^{*}$ para el ligando libre), ocurren típicamente en el ultravioleta $(\lambda<350 \mathrm{~nm})$ y poseen un alto coeficiente de extinción $\left(\varepsilon \geq 10^{4} \mathrm{M}^{-1} \mathrm{~cm}^{-1}\right)$.

Figura 1: Descripción cualitativa de los orbitales moleculares del $\mathrm{Ru}\left(2,2^{\prime} \mathrm{bipi}\right)_{3}{ }^{+2}$ y los tipos de transiciones electrónicas posibles: $(-)$ TCML, $(-)$ TCLM, $(-)$ IL, $(-$ CM y $(-)$ TCS

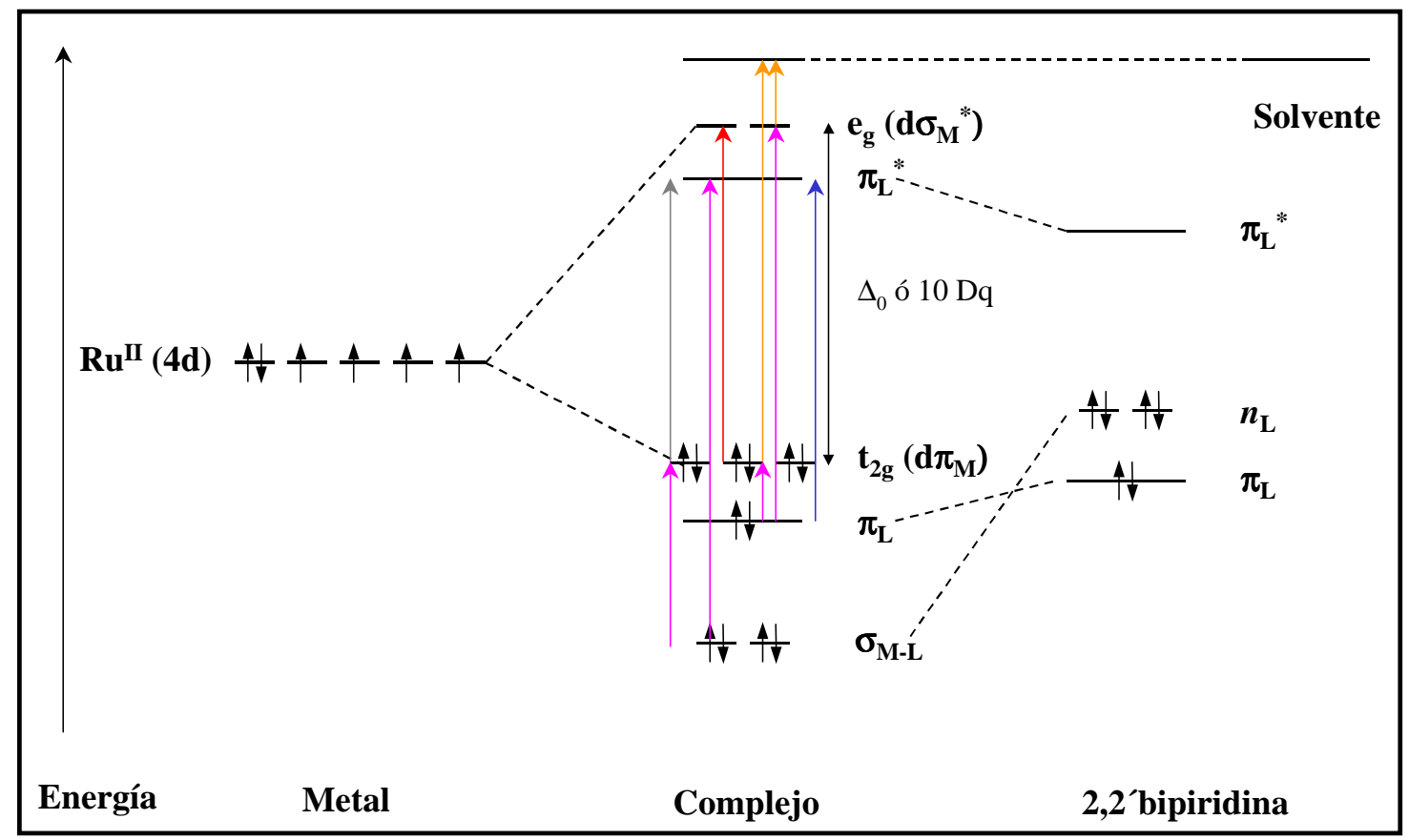

Otro tipo de transición que puede ocurrir, observando la figura 1, es aquella en la cual están involucrados los orbitales $d$ del metal y se denominan transiciones centradas en el metal, CM ó transiciones $d \rightarrow d, t_{2 g} \rightarrow e_{g}$ en la figura 1. Estas transiciones están formalmente prohibidas en una geometría octahédrica resultando en absorciones muy débiles $\left(\varepsilon \sim 10-100 \mathrm{M}^{-1} \mathrm{~cm}^{-1}\right)$, usualmente en la región visible. Como estas transiciones involucran la promoción de un electrón hacia un orbital de naturaleza antiligante $\left(\mathrm{d} \sigma^{*}\right.$-metal) el enlace metal-ligando se debilita y por lo tanto, las transiciones $\mathrm{d} \rightarrow \mathrm{d}$ comúnmente resultan en reacciones fotoquímicas de sustitución de ligandos. Los complejos tricarbonílicos de $\mathrm{Re}^{\mathrm{I}}$ prácticamente 
no experimentan este tipo de transiciones debido al gran efecto de campo ligando que producen los carbonilos. Por lo tanto, dichos orbitales se encuentran a mayor energía y, como consecuencia de ello, presentan una notable estabilidad en relación con el cambio de ligandos. De esta manera, las absorciones que se observan, en los carbonilos de $\mathrm{Re}^{\mathrm{I}}$, corresponden, generalmente, a las transiciones fuertemente permitidas TCML e IL.

\section{Fotofísica}

Las características de los estados excitados del $\mathrm{Ru}\left(2,2^{\prime} \text { bipi }\right)_{3}{ }^{+2}$ pueden ser resumidas y representadas en un diagrama de Jablonski, figura 2. En general, se puede considerar que las moléculas del complejo en su estado basal, presentan una distribución electrónica en donde todos sus electrones se encuentran apareados, es decir, son de naturaleza singletes (la multiplicidad global de la molécula es igual a 1). Si la promoción de un electrón a algún estado excitado de energía mayor, luego de la absorción de luz, se produce manteniendo las coordenadas nucleares del estado fundamental, es decir, los cambios electrónicos ocurren mucho más rápidamente que los rearreglos nucleares, entonces estas transiciones electrónicas se dice que obedecen al principio de Franck-Condon, y a los estados generados inmediatamente después de la absorción de luz se los denomina estados de Franck-Condon. Debido a que las transiciones en las cuales se mantiene la misma multiplicidad de spin son completamente permitidas (y por lo tanto las absorciones serán intensas) se asume que los estados excitados poblados también serán de naturaleza singletes. Una transición a un estado triplete (en la cual el electrón promovido sufrió un cambio de spin) es prohibida por spin y por lo tanto existirá una baja probabilidad de poblar ese estado desde uno basal singlete. Por lo tanto las absorciones, si las hubiera, serán muy débiles. Sin embargo, como veremos más adelante, estos estados se pueden poblar siguiendo otros caminos. 
Para el complejo modelo considerado, al excitar con luz de $450 \mathrm{~nm}$, se pueblan estados singletes de transferencia de carga, ${ }^{1} \mathrm{TCML}$, generalmente el correspondiente al de menor energía $\left(\mathrm{S}_{1}\right)$, aunque otros de niveles más altos se pueden poblar también en forma directa $\left(\mathrm{S}_{2}\right.$, $\mathrm{S}_{3}, \ldots$ etc). En estos casos, luego de la absorción, se produce un proceso de relajación rápida, antes que cualquier otro proceso, hacia el estado excitado más bajo ${ }^{1}$ TCML (ley de Kasha), debido a la reorganización de los enlaces en toda la estructura molecular producto de los cambios en la configuración electrónica. Este paso de disipación de energía en forma no radiativa se la denomina conversión interna y ocurre siempre entre estados de igual multiplicidad. La constante de velocidad específica que caracteriza este proceso de denomina $k_{i c}$ y generalmente es del orden de $10^{13} \mathrm{~s}^{-1}$, tanto en sistemas orgánicos como inorgánicos.

El estado excitado ${ }^{1}$ TCML puede continuar ahora su camino de regreso al estado de energía basal $\left(\mathrm{S}_{0}\right)$ siguiendo pasos de desactivación radiativos y no radiativos. El camino radiativo, es decir, la pérdida de energía por emisión de luz, se conoce como fluorescencia cuando los estados involucrados poseen la misma multiplicidad $\left(\mathrm{S}_{1} \rightarrow \mathrm{S}_{0}\right)$, siendo $k_{f}$ la constante de velocidad. Tanto en sistemas orgánicos como inorgánicos $k_{f}$ toma valores del orden de $10^{8} \mathrm{~s}^{-1}$. Se denomina cruzamiento intersistemas a una transición no radiativa entre estados de distinta multiplicidad $(\Delta S=1)$ la cual está formalmente prohibida por spin. Esta regla se cumple, en general, en moléculas orgánicas que contienen átomos de bajo número atómico y por lo tanto estos procesos son muy lentos (la constante de velocidad específica, $k_{i s c}$, es del orden 1$\left.1000.10^{6} \mathrm{~s}^{-1}\right)$. En sistemas inorgánicos, en cambio, las diferencias pueden ser sustanciales. Estas diferencias se deben, en parte, a los altos valores de las constantes de acoplamiento spin-órbita asociadas con los altos pesos atómicos de los iones metálicos. El incremento del acoplamiento spin-orbital causa un efecto de mezcla entre los estados singletes y tripletes lo cual, de alguna manera, atenúa (flexibiliza) la regla de selección anterior. Por este motivo, 
estados tripletes pueden poblarse eficientemente desde estados singletes cercanos más altos ${ }^{1)}$ y las $k_{i s c}$ correspondientes serán del orden de $10^{9}-10^{12} \mathrm{~s}^{-1}$. Así, estas transiciones son muy rápidas e indistinguibles de la conversión interna la cual no tiene ninguna prohibición.

Figura 2: Diagrama de Jablonski que representa los procesos de desactivación de los estados excitados del complejo $\mathrm{Ru}\left(2,2^{\prime} \text { bipi }\right)_{3}{ }^{+2}$. $(-)$ caminos radiativos

\section{$(-)$ caminos no radiativos}

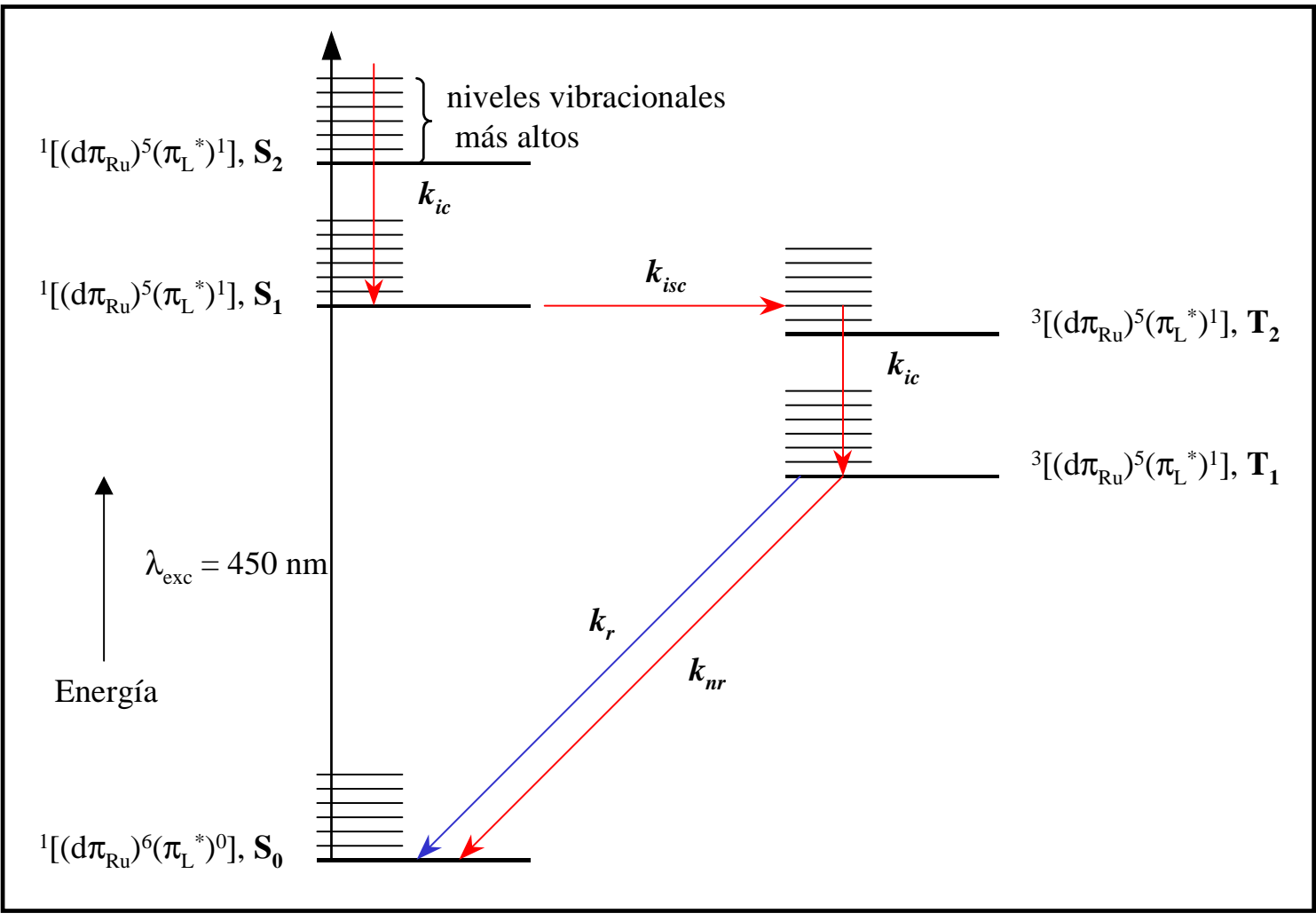

El estado ${ }^{3} \mathrm{TCML}$ de más baja energía, $\mathrm{T}_{1}$, es el estado excitado responsable, en esta familia de complejos, de la luminiscencia y reactividad química que se monitorean en las medidas de emisión en estado estacionario, emisión resuelta en el tiempo y experimentos de flash

\footnotetext{
1) En general se verifica que los estados tripletes se encuentran a una energía menor con respecto al correspondiente estado singlete debido a que los estados de mayor multiplicidad son más estables ya que su configuración electrónica presenta una mínima energía de repulsión electrón-electrón.
} 
fotólisis. En efecto, una vez poblado, la desactivación de este estado para retornar al estado basal puede seguir un camino no radiativo por el cual la energía es disipada como calor hacia los alrededores, a través de vibraciones moleculares internas de altas frecuencias. Este proceso posee una constante de velocidad específica denominada $k_{n r}$. Además, la emisión espontánea de un fotón de luz puede ocurrir desde este estado ${ }^{3}$ TCML, siguiendo un camino radiativo de desactivación con constante $k_{r}$. En sistemas orgánicos, a este paso radiativo en el cual los estados involucrados poseen diferente multiplicidad de spin se lo denomina fosforescencia $\left(\mathrm{T}_{1} \rightarrow \mathrm{S}_{0}\right)$ y la constante de velocidad es $k_{p}$. Este modo de desactivación está prohibido por spin y por lo tanto $k_{p}$ toma valores entre $0,02-100 \mathrm{~s}^{-1}$ permitiendo hacer una clara distinción fenomenológica, entre fluorescencia y fosforescencia, mediante los tiempos de vida de la emisión. En sistemas inorgánicos esta distinción puede ser incierta debido a la característica de mezcla que poseen los estados excitados y $k_{p}$ puede tomar valores entre $10^{2}-$ $10^{7} \mathrm{~s}^{-1}$. Por esta razón en complejos de metales de transición se utiliza el término luminiscencia que describe el camino radiativo correspondiente. Se puede asumir, como regla para complejos polipiridínicos de metales de transición $\mathrm{d}^{6}$, que el decaimiento no radiativo domina y es más importante que el radiativo.

\section{i. Decaimiento radiativo}

El análisis del decaimiento radiativo es muy importante para establecer la dinámica de los estados excitados y provee, usualmente, el primer indicio sobre el carácter del/los estado/s excitado/s de más baja energía en esta familia de complejos. La emisión típica del estado excitado TCML corresponde a una banda ancha y sin estructura a temperatura ambiente en solución. La forma de la banda de emisión, su máximo de energía $\mathrm{E}_{\mathrm{emi}}$, rendimiento cuántico $\phi_{\text {emi }}$, tiempo de vida $\tau_{\text {emi }}$, etc., son datos experimentales directamente medibles, como se 
describirá más adelante. El rendimiento cuántico de emisión, $\phi_{\mathrm{emi}}=k_{r} /\left(k_{r}+k_{n r}\right)$ dependiente de la longitud de onda de excitación, indica un eficiente cruzamiento entre sistemas para este tipo de complejos, es decir, el pasaje de ${ }^{1}$ TCML $\stackrel{k_{i s c}}{\longrightarrow}{ }^{3}$ TCML se produce con eficiencia 1. Como se mencionó anteriormente, la presencia de un átomo "pesado" permite una rápida formación del ${ }^{3}$ TCML desde el ${ }^{1}$ TCML como consecuencia de la alta constante de acoplamiento spin-órbita, impartiendo algún carácter singlete al estado ${ }^{3}$ TCML. Por esta razón, la denominación de estados singlete y triplete para estos complejos no es estrictamente correcta aunque el formalismo se mantiene. El tiempo de vida del estado excitado emisor está relacionado con la inversa de la suma de las constantes correspondientes a los pasos de decaimiento, $\tau_{\mathrm{emi}}=\left(k_{r}+k_{n r}\right)^{-1}$. Como se mencionó anteriormente, la desactivación del estado excitado está dominado por el mecanismo no radiativo, es decir $k_{n r} \gg k_{r}$ y por lo tanto $\tau_{\mathrm{emi}} \cong$ $1 / k_{n r}$. De esta manera se podría calcular fácilmente $k_{r}$ y $k_{n r}$ una vez determinados experimentalmente el $\phi_{\mathrm{emi}} \mathrm{y} \tau_{\mathrm{emi}}$. Siguiendo con el decaimiento del estado excitado ${ }^{3} \mathrm{TCML}$ al estado fundamental singlete, nuevamente vemos que este paso está formalmente prohibido por spin, pero el mismo efecto de acoplamiento spin-órbita permite un decaimiento luminiscente del estado excitado con tiempos de vida del orden de 0,1 - $2 \mu$ s para cromóforos como el $\mathrm{Ru}\left(2,2^{\prime} \text { bipi }\right)_{3}{ }^{+2 *}$. Estos tiempos son relativamente de vida larga comparado con los de transiciones singlete-singlete puras de sistemas orgánicos cuyo $\tau_{\mathrm{emi}} \sim 1 \mathrm{~ns}$ (fluorescencia), pero más cortos que los tiempos en que se efectúa una transición fosforescente, que son del orden del milisegundo, para estos mismos sistemas.

\section{ii. Efectos del solvente}

La emisión originada en un estado excitado TCML es también sensible a la polaridad del solvente. El estado excitado genera un gran cambio en el momento dipolar con respecto al 
estado fundamental producto de la transferencia de un electrón desde el metal hacia el ligando alojándose ahora sobre esta parte del complejo. Esto provoca una mayor estabilización en solventes más polares. El origen microscópico de esto, está relacionado con el efecto que sobre la constante dieléctrica tiene el solvente y pueden ser racionalizados en el siguiente contexto: cuando el estado excitado de más baja energía ${ }^{3}$ TCML sigue los pasos de relajación, las moléculas del solvente se reorientarán en función del momento dipolar generado por el estado excitado. Los solventes más polares podrían producir una mayor reorientación de sus moléculas generando un efecto de mayor estabilización en el estado excitado. Obviamente, para que esta dinámica tenga lugar, la redistribución del solvente debe ocurrir mucho más rápidamente que el decaimiento del estado excitado. Un rasgo interesante de esta dinámica del solvente es que bajo condiciones de baja temperatura (120 - $140 \mathrm{~K}$ en mezclas de etanol metanol 4:1 ( $\mathrm{vol} / \mathrm{vol}), 4: 1 \mathrm{EtOH} / \mathrm{MeOH})$ la solución se encuentra en un estado intermedio vidrio-fluido y los cambios en la reorganización del solvente ocurren en una escala de tiempo similar a la del decaimiento del estado excitado, observándose cinéticas no exponenciales. Al enfriar hasta $77 \mathrm{~K}$ la solución se transforma en una matriz rígida de vidrio y la cinética de tipo exponencial para el decaimiento del estado excitado reaparece. Sobre la energía de la emisión se produce un dramático corrimiento hacia el azul producto del denominado efecto "rigidocrómico". Otro rasgo común de los espectros a baja temperatura es la observación de emisión estructurada.

\section{iii. $\quad$ Emisión $^{3}\left(\pi^{*} \rightarrow \pi\right)$}

A pesar de la prevalencia de la emisión del estado excitado TCML a temperatura ambiente, otro tipo de emisión ha sido observada en complejos de metales de transición d d $^{6}$ En particular para los complejos tricarbonílicos de $\mathrm{Re}^{\mathrm{I}}$ con ligandos bidentados de tipo diimina, la emisión 
${ }^{3}\left(\pi^{*} \rightarrow \pi\right)$ se origina en un estado de tipo IL del complejo; se observa más claramente a $77 \mathrm{~K}$ y puede ser fácilmente asignada por la fuerte similitud con el espectro de fosforescencia del ligando no coordinado. Estos ligandos muestran estructura vibrónica similar a la de los espectros de emisión del TCML a 77 K, sin embargo el máximo de emisión de TCML está corrido más hacia el rojo $\left(\sim 2500 \mathrm{~cm}^{-1}\right)$ que el $\mathrm{del}^{3}\left(\pi^{*} \rightarrow \pi\right)$ comparado con la fosforescencia del ligando. La banda de emisión es angosta y estructurada aún a temperatura ambiente aunque muchas veces se encuentra enmascarada por la emisión coexistente de la transferencia de carga. Los estados ${ }^{3} \mathrm{IL}$ son usualmente de vida larga aunque sus tiempos de vida son más cortos comparados con los del ligando libre. Estos estados muestran una pequeña ó nula dependencia con el solvente debido a que no se produce un gran cambio en el momento dipolar cuando el estado excitado IL es generado. La introducción en los complejos de ligandos polipiridínicos con estructuras aromáticas que poseen un sistema $\pi$ extensamente deslocalizado, aumenta la posibilidad de generar estados excitados de energía más baja centrados en el ligando. Ejemplos de ellos se encuentran en la bibliografía para diferentes complejos de $\operatorname{Re}^{\mathrm{I}}$.

\section{iv. Quenching bimolecular}

Otro método para la desactivación del estado excitado emisor ${ }^{3}$ TCML es por transferencia de electrones ó energía hacia donores ó aceptores apropiados. Cuando se produce la transferencia de carga metal-ligando, el electrón se ubica en un orbital $\pi^{*}$ de alta energía del ligando, y a la vez, se genera un hueco en el orbital de baja energía d $\pi$ del metal. Por lo tanto el potencial de ionización decrece y la afinidad electrónica se incrementa cuando la molécula pasa del estado electrónico fundamental al estado electrónico excitado. Esto quiere decir que un estado electrónico excitado, en general, será un mejor reductor y un mejor oxidante que el estado 
electrónico fundamental. Por lo tanto, en presencia de aceptores y donores de electrones ocurre una rápida transferencia de electrones que desactiva el estado excitado ${ }^{3}$ TCML. En complejos tricarbonílicos de $\operatorname{Re}^{\mathrm{I}}$ han sido ampliamente utilizadas aminas reductoras como la trietanolamina (TEOA) y trietilamina (TEA). Como ejemplo se muestra en la ecuación siguiente la reacción entre TEOA y un complejo de $\operatorname{Re}^{\mathrm{I}}$ con ligandos 4-fenilpiridina (4-phpy): $\operatorname{Re}(\mathrm{CO})_{3}\left(4-\right.$ phpy $_{3}{ }^{+^{*}}+\mathrm{TEOA} \stackrel{k_{q}}{\longrightarrow} \operatorname{Re}(\mathrm{CO})_{3}(4-\text { phpy })_{3}{ }^{\bullet}+\mathrm{TEOA}^{+}$

Posteriormente, la recombinación rápida de los productos regenera el estado fundamental. El estado excitado ${ }^{3}$ TCML también puede ser empleado en reacciones de sensibilización por transferencia de energía a estados tripletes de moléculas orgánicas como ocurre entre el estado excitado del complejo $\mathrm{Ru}\left(2,2^{\prime} \text { bipi }\right)_{3}{ }^{+2}$ y el antraceno.

\section{Fotoquímica}

La fotoquímica de estos complejos de metales de transición y en particular los que tienen al $\operatorname{Re}^{\mathrm{I}}$ como metal central, involucra, en la mayoría de los casos, reacciones de transferencia de electrones del estado excitado ${ }^{3} \mathrm{TCML}$ debido, fundamentalmente, como ya se mencionó, a que son fuertes oxidantes y reductores comparado con el estado fundamental. Los estados excitados de estos complejos son usualmente de vida larga (ns- $\mu$ s) lo que le permite participar en reacciones rápidas de transferencia de electrones ó de energía similares a las que se describieron anteriormente. A pesar de que los complejos de $\operatorname{Re}^{\mathrm{I}}$ poseen mayor estabilidad fotoquímica que otros complejos parientes, ante la pérdida de ligandos, se han informado algunos ejemplos de este tipo de reacciones, por ejemplo, para el $\operatorname{Re}(\mathrm{CO})_{3}(4-\mathrm{phpy})_{3}{ }^{+}$ excitando con pulsos de luz de $355 \mathrm{~nm}$ :

$$
\operatorname{Re}(\mathrm{CO})_{3}\left(4-\text { phpy }_{3}{ }^{+}+\mathrm{hv} \longrightarrow \operatorname{Re}(\mathrm{CO})_{3}(4-\text { phpy })_{2}{ }^{+2}+\text { 4-phpy }{ }^{\bullet}\right.
$$


Por otro lado la alta reactividad de las especies reducidas puede ser utilizada en fotocatálisis. Por ejemplo, la fotoexcitación del complejo $\mathrm{ClRe}(\mathrm{CO})_{3}\left(2,2^{\prime}\right.$ bipi $)$ en presencia de un quencher reductivo como la TEOA produce el radical $\mathrm{ClRe}(\mathrm{CO})_{3}\left(2,2^{\prime} \text { bipi }\right)^{\bullet-}$ el cual pierde el ligando $\mathrm{Cl}^{-}$. Una reacción posterior con $\mathrm{CO}_{2}$ presente en la solución resulta en una reducción fotocatalizada del $\mathrm{CO}_{2}$ para dar $\mathrm{CO}$ vía un complicado mecanismo de reacción. Similar resultado se obtuvo utilizando como fotocatalizador el complejo $\mathrm{ClRe}(\mathrm{CO})_{3}(4-\mathrm{phpy})_{2}$.

La oxidación del estado excitado, las reacciones de transferencias de electrones intramoleculares ó estudios sobre la dinámica de fragmentación de un ligando por oxidación, son otras de las maneras en que estos complejos muestran su variada y versátil reactividad fotoquímica. También fue observado un tipo completamente diferente de fotoreactividad que involucra una ruptura homolítica de un enlace Re-ligando produciendo radicales que podrían encontrar aplicaciones en la fotoiniciación de reacciones de polimerizaciones por radicales.

Obviamente, la fotoquímica y fotofísica de estos complejos dependerá fuertemente de la composición de la esfera de coordinación, es decir, de la naturaleza particular de los ligandos. Las reacciones correspondientes a estados centrados en el metal ó dd para esta familia de compuestos son de menor importancia fotoquímica debido a que estos estados se encuentran a una energía mayor.

\section{Monitoreo de los procesos del estado excitado}

A continuación se describen los equipos y técnicas empleadas para la determinación de los factores que influyen en la dinámica del estado excitado. También se mencionan otras técnicas complementarias y algunas de las utilizadas para la caracterización de los complejos. 
Igualmente, en cada capítulo en los que se presentan los resultados de los experimentos realizados, se menciona en forma resumida los equipos utilizados para cada medida en particular y otras especificaciones experimentales. 


\section{Método de flash fotólisis}

\section{i. $\quad$ Descripción general del método y detalles experimentales}

El método de flash fotólisis fue desarrollado por R. G. W. Morrish y G. Porter en 1948. Es un método utilizado para la iniciación y el estudio de procesos fotoquímicos. En esta técnica se crea una situación de no-equilibrio en la mezcla de reacción en un intervalo de tiempo corto, generando intermediarios inestables en concentraciones más altas que las que pueden ser obtenidas por otros métodos.

El procedimiento consiste en someter al material en estudio a un corto e intenso flash de luz con lo que se forma una concentración relativamente alta de moléculas excitadas y/ó radicales libres. Las especies transitorias producidas son detectadas por espectroscopía de absorción ó de emisión, pudiéndose seguir la evolución temporal de las mismas.

Al producirse la absorción de luz por una molécula se originan especies en estados electrónicos excitados. Dichas especies pueden sufrir una transformación química permanente es decir, una reacción fotoquímica, o volver al estado fundamental del cual partieron a través de decaimiento radiativo y/ó no radiativo. Estos procesos de decaimiento cubren un amplio intervalo de tiempos desde los picosegundos (para la relajación vibracional en solución), nanosegundos (para los procesos radiativos entre estados de igual multiplicidad), microsegundos (para los procesos radiativos entre estados de distinta multiplicidad) y en algunos casos hasta segundos (para algunos radicales y especies químicas de vida larga).

Los estudios fotoquímicos resultan de gran importancia ya que han permitido investigar numerosas reacciones químicas, sus mecanismos y la naturaleza de los intermediarios involucrados. Además su aplicación al estudio de reacciones rápidas se basa en el hecho de 
que la iniciación fotoquímica es muy conveniente para producir una reacción química en forma homogénea, en un corto intervalo de tiempo.

La resolución temporal está limitada por la duración del flash, que es usualmente de unos pocos microsegundos en el caso de lámparas de plasma (flash fotólisis convencional). También, la intensidad del flash debe ser suficientemente alta como para producir un cambio medible en la composición química, pero de muy corta duración comparada con la vida media de los procesos que quieren estudiarse. Estas, son dos de las limitaciones más importantes de esta técnica, cuando se desea estudiar intermediarios ó estados excitados de vida muy corta, usando lámparas pulsadas como fuente de excitación.

El desarrollo y aplicación de fuentes de luz láser, a fines de la década del `60, dio inicio a la técnica de láser flash fotólisis. El descubrimiento de los láseres pulsados proveyó una nueva fuente para la excitación, posibilitando la extensión del método de flash fotólisis desde los microsegundos a las regiones de los nanosegundos, picosegundos y hasta femtosegundos.

En todos los láseres, la absorción de energía proveniente de una fuente externa eleva a un número suficiente de moléculas desde su estado fundamental a un estado excitado de modo de permitir que ocurra una inversión de la población a ciertos niveles de energía favorecidos. La emisión espontánea de un fotón de alguna de las moléculas excitadas estimula la emisión de otras emitiendo en fase y cuando la cascada de energía aumenta muy rápidamente, ocurre la acción láser (amplificación de luz por emisión de radiación estimulada, LASER en inglés), produciéndose un haz de radiación intenso y coherente, de ancho de banda pequeño. De esta manera, las características de la luz de excitación láser y que son una ventaja con respecto a las lámparas de plasma, son las siguientes:

1. las potencias de pico pueden ser enormes $\left(10^{9}\right.$ a $10^{12}$ watts $)$ 
2. la luz emitida es coherente (los fotones están en fase), monocromática (porque los fotones estimulan fotones de la misma frecuencia), y puede ser enfocada en áreas muy pequeñas

3. el pulso de luz es generalmente simétrico con el tiempo y no está acompañado por una larga cola

4. la duración del pulso luminoso es muy corto (nano al femtosegundo)

Existen una gran cantidad de tipos de láseres que emiten a longitudes de onda característica abarcando amplias regiones del espectro electromagnético, desde los $193 \mathrm{~nm}$ hasta la décima de $\mu \mathrm{m}$. También, la energía y duración del pulso de luz difiere en cada caso, así como la naturaleza de la especie emisora. En función de estos parámetros, los láseres, que pueden ser continuos ó pulsados, se clasifican en varios grupos.

Los láseres de gas continuos de modo acoplado, como los de helio-neón, helio-cadmio, argón, y kriptón, producen pulsos menores que el nanosegundo, repetitivos, de relativamente baja energía, que son apropiados para los estudios de emisión. Los láseres de gas pulsados como los de nitrógeno, hidrógeno y monóxido de carbono emiten pulsos de luz ultravioleta de hasta unos cientos de kilowatts de potencia, con anchos de pulso de unos pocos nanosegundos. Láseres pulsados de dióxido de carbono de alta potencia emiten en la región del infrarrojo, cerca de 10,6 $\mu \mathrm{m}$. Los láseres de semiconductores, que pueden ser continuos o pulsados, emiten generalmente bajas potencias en el infrarrojo. Los láseres de líquidos, que incluyen los de colorantes, pueden producir pulsos cuya energía se encuentra entre el ultravioleta cercano y el infrarrojo. Los láseres de estado sólido (rubí, neodimio) pueden producir pulsos de luz de muy alta potencia, y son los que han encontrado el uso más extensivo en flash fotólisis. Para excitar en la región UV, son extremadamente importantes y utilizados los denominados láseres de excímeros, ya que emiten con pulsos de luz de muy alta energía (35 a 200 mJ / 
pulso). Están compuestos por una mezcla de un gas halógeno con un gas noble y la energía, ancho de pulso y frecuencia de emisión dependen de la composición de la mezcla gaseosa.

En la mayoría de los experimentos de láser flash fotólisis realizados en este trabajo de tesis se utilizó un láser de excímero de $\mathrm{XeF}, \lambda_{\mathrm{exc}}=351 \mathrm{~nm}$. El equipo trabaja en modo cinético, es decir, registrando los cambios de absorción ó densidad óptica $(\Delta \mathrm{DO})$ en función del tiempo, para las especies transitorias originadas por el pulso de luz, a una longitud de onda fija. Esto se realiza utilizando un haz luminoso proveniente de una fuente de luz de análisis ó monitoreo que se colima, se hace pasar a través de la celda de reacción y, luego de atravesar un monocromador, es detectado por un fotomultiplicador y registrado en un osciloscopio. Los espectros de absorción de los estados excitados se obtienen a partir de la determinación de los $\Delta \mathrm{DO}$ a un tiempo fijo, de las trazas obtenidas para cada longitud de onda.

Para el monitoreo de la radiación emitida por los estados excitados emisores, se utilizó un láser de nitrógeno, $\lambda_{\mathrm{exc}}=337 \mathrm{~nm}$.

En ambos casos, el arreglo óptico utilizado presentaba un diseño a través del cual el pulso de excitación del láser incide en la celda conteniendo la muestra con un ángulo de $90^{\circ}$ con respecto al tren óptico de detección de la señal (y de la luz de análisis para las medidas de $\Delta \mathrm{DO})$ 


\section{ii. $\quad$ Componentes del equipo de láser flash fotólisis}

En general, un equipo de láser flash fotólisis debe incluir los componentes mostrados en la figura 3:

1. una celda que contiene la muestra, que puede ser de geometría y material variable dependiendo del tipo de láser utilizado y del volumen de muestra utilizado ${ }^{1)}$. Para los experimentos presentados en este trabajo, se utilizaron celdas de cuarzo de calidad óptica de cuatro ventanas $(1 \mathrm{~cm} \mathrm{x} 1 \mathrm{~cm} \times 4 \mathrm{~cm}$ de altura). En el extremo superior, la celda posee un robinete para permitir la deaereación de la solución antes, y/ó durante, la medida. Cuando las condiciones de trabajo así lo requirieron, se utilizó una celda similar que permitía el flujo continuo de solución (a una velocidad adecuada) de manera de trabajar constantemente con solución fresca ( $\sin$ fotolizar).

2. una fuente de luz de análisis, que típicamente puede ser una lámpara de arco de Xe con potencia de la luz emitida dentro del rango de 150 y 1000 watts ó de tungsteno-halógeno. La luz de análisis es convenientemente colimada y enfocada sobre la celda de reacción, entrando usualmente por una pequeña ventana u orificio (1 ó $2 \mathrm{~mm})$. La intensidad de la luz de análisis, para el registro en el nanosegundo, debe ser alta de modo de producir una señal fotométrica con adecuada relación señal / ruido y reducir los efectos de la fluorescencia dispersada.

3. una fuente de excitación láser cuya elección deberá hacerse teniendo en cuenta, fundamentalmente, la energía, longitud de onda de excitación y duración del pulso. El valor de la energía requerida dependerá de los coeficientes de extinción de los estados excitados y/ó

\footnotetext{
1) Esta técnica puede ser aplicada a gases y líquidos, en un amplio rango de temperaturas y concentraciones. Para nuestros experimentos, todas las muestras fueron soluciones líquidas en solventes orgánicos y a temperatura ambiente, si no se aclara otra condición.
} 
intermediarios de reacción bajo estudio y de sus rendimientos cuánticos de formación. Un sistema versátil es aquel que permite trabajar en un rango entre 1 y $100 \mathrm{~mJ} /$ pulso. La duración del pulso debe ser cuidadosamente seleccionada. Un ancho de pulso entre 5 y 20 ns es común y usualmente aceptado. El $\lambda_{\text {exc }}$ dependerá, fundamentalmente, de la naturaleza del láser en sí mismo (como se describió anteriormente). El haz del láser se concentra sobre la celda con geometría de $90^{\circ}$ cubriendo totalmente el camino óptico del haz de análisis y la imagen de salida de la celda se proyecta sobre la rendija de un monocromador.

4. un sistema de lentes y filtros adecuado para colimar, dirigir y enfocar el haz de luz de análisis y el pulso de la luz de excitación. Los filtros comúnmente usados son de paso de banda, de vidrio de calidad óptica ó soluciones de sustancias que absorben la luz del láser atenuando su energía. Si bien se pueden emplear otros arreglos geométricos la excitación a $90^{\circ}$ es fácil de alinear y asegura bajos niveles de dispersión.

5. un detector de la señal luminosa que consiste típicamente de una combinación de un monocromador y un tubo fotomultiplicador. El monocromador es frecuentemente de tipo holográfico y cubre el rango de 230 a $800 \mathrm{~nm}$. El fotomultiplicador toma la señal a la salida del monocromador y genera una señal eléctrica que se amplifica y, controlando el voltaje aplicado, se ajusta a la región de respuesta lineal.

6. un osciloscopio digitalizador que recibe la señal del fotomultiplicador y la convierte a la forma digital y transfiere estos datos a una computadora para el procesamiento posterior (análisis y almacenamiento de los datos). 
Figura 3: Arreglo experimental y componentes de un equipo de láser flash fotólisis.

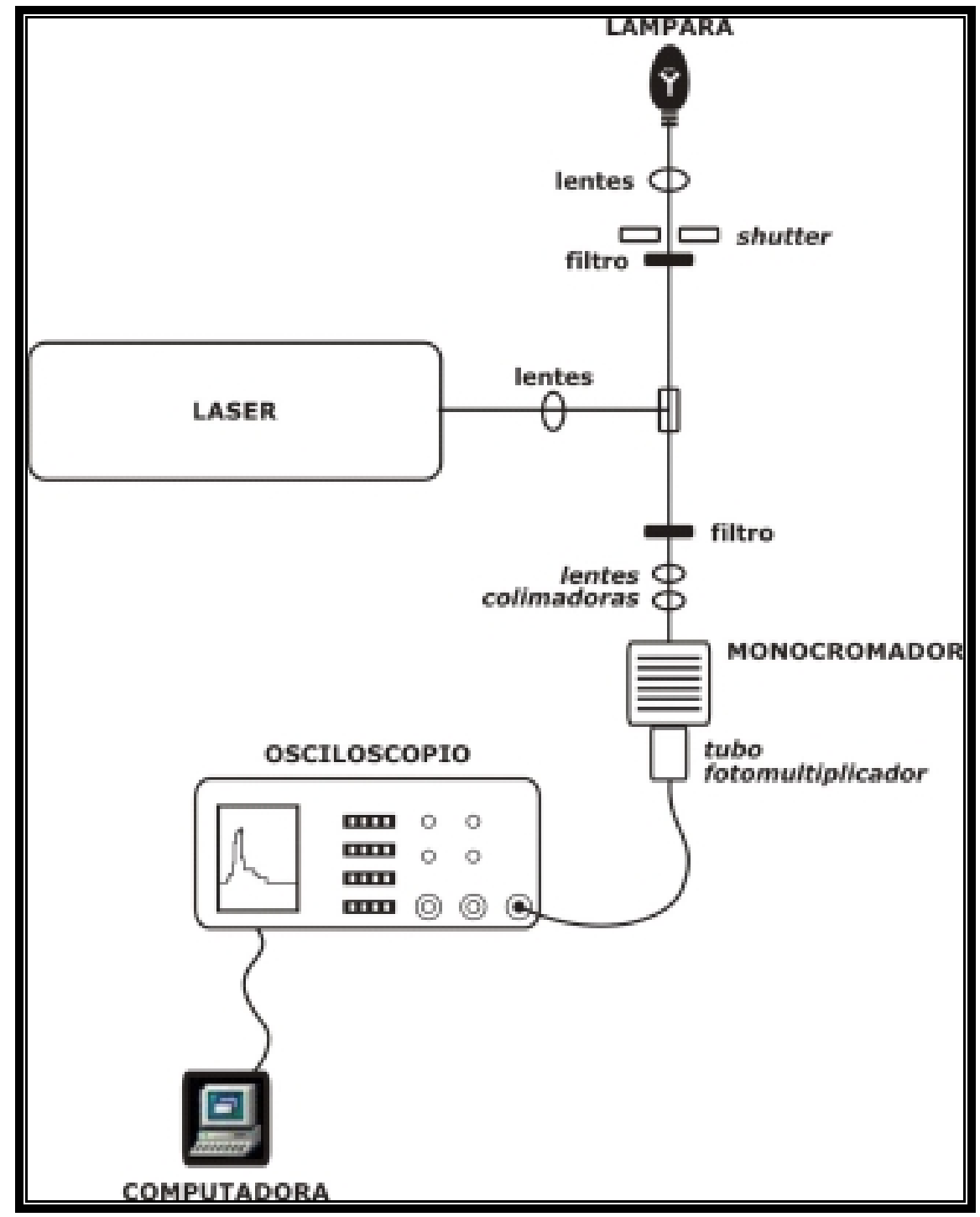

\section{iii. Procesamiento de los datos}

La señal registrada en el osciloscopio de un sistema de láser flash fotólisis consiste en una representación en función del tiempo de la intensidad de la luz de análisis constituida por algunos cientos de puntos conteniendo la señal de voltaje del fotomultiplicador. Si durante el 
proceso estudiado se forma una especie transitoria que absorbe más luz que la solución de partida a la longitud de onda de análisis, el osciloscopio registrará una señal del tipo de la que se muestra en la figura 4. En el caso contrario, es decir, cuando la absorción de la especie transitoria es menor que la del material de partida, se pueden registrar señales que corresponden a absorciones negativas ("bleaching"). Es posible también obtener señales en las cuales la absorbancia final sea mayor a la absorbancia antes del pulso del láser, es decir, que no coincida con la línea de base debido a la absorción de fotoproductos ó especies transitorias de vida más larga. En este punto se debe enfatizar que las medidas no corresponden a valores absolutos de la densidad óptica sino a los cambios de la absorción donde el valor cero corresponde a la absorción del material de partida.

Para transformar y obtener entonces la señal correspondiente a la diferencia en la densidad óptica del sistema, $\Delta \mathrm{DO}$, se procede como se describe a continuación. Debe aclararse que, como la especie transitoria absorbe sólo una pequeña fracción de luz, es necesario amplificar la ganancia del osciloscopio, en cuyo caso la posición correspondiente al 0\% de transmitancia no aparecerá en la pantalla del mismo. La respuesta del osciloscopio, x, es proporcional a la intensidad luminosa, I; por lo tanto, pueden plantearse las siguientes relaciones:

$$
\mathrm{I}_{\mathrm{o}}=\mathrm{k} \cdot \mathrm{x}_{\mathrm{o}} \quad \mathrm{I}=\mathrm{k} \cdot \mathrm{x}
$$

donde $\mathrm{k}$ es una constante que depende de la sensibilidad del detector. Combinando estas ecuaciones en la ecuación de la ley de Lambert-Beer puede escribirse la siguiente relación:

$$
\mathrm{A}=\log \left(\frac{\mathrm{I}_{0}}{\mathrm{I}}\right)=\log \left(\frac{x_{0}}{x}\right)=\varepsilon_{\lambda} \mathrm{C}_{\mathrm{t}} l
$$

donde $\varepsilon_{\lambda}$ es el coeficiente de extinción de la especie transitoria a la longitud de onda $\lambda, \mathrm{C}_{\mathrm{t}}$ es 
su concentración al tiempo t después del pulso del láser y $l$ es el camino óptico.

Figura 4: Esquema de una traza obtenida en un experimento de láser flash fotólisis en la que se observa la desaparición (decaimiento) de una especie transitoria formada luego del pulso del láser.

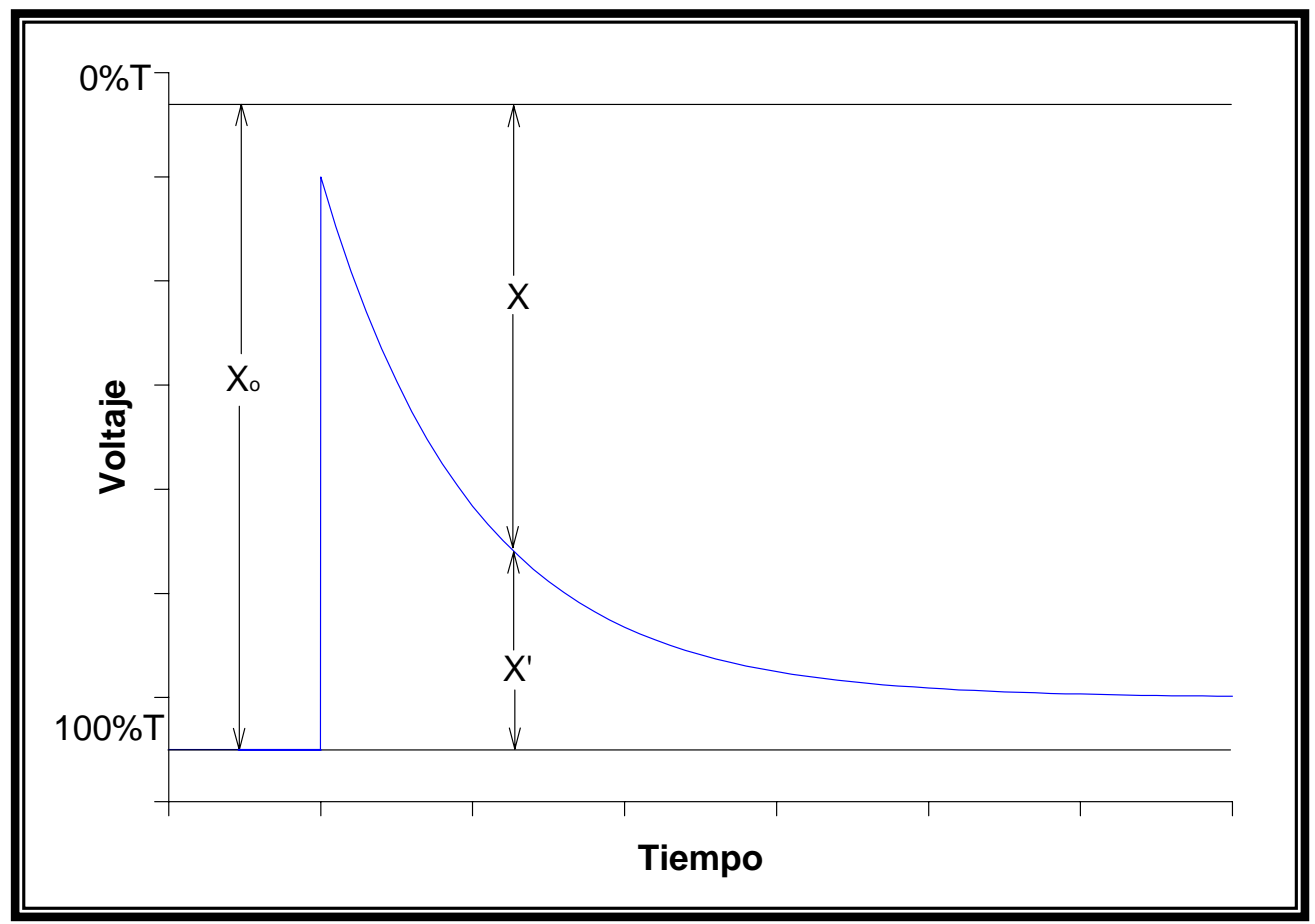

En la figura 4 se observa que la amplitud de la señal x está dada en unidades de voltaje. Con el fin de convertir estos valores en absorbancia o transmitancia porcentual, se ajusta a 1 voltio el $100 \%$ de transmitancia de la luz de monitoreo utilizando un comparador diferencial conectado al osciloscopio. Como la línea correspondiente a $0 \%$ de transmitancia no se registra, no se obtienen las amplitudes X para cada intervalo de tiempo, pero sí las que fueron marcadas como X' en el esquema de la figura 4. La relación entre x y X' viene dada por:

$$
X^{\prime}=X_{0}-X
$$


Puede plantearse entonces, teniendo en cuenta que $\mathrm{x}_{\mathrm{o}}=1000 \mathrm{mV}$, la siguiente ecuación:

$$
\mathrm{A}=\log \left(\frac{\mathrm{I}_{0}}{\mathrm{I}}\right)=\log \left(\frac{x_{0}}{x_{0}-x^{\prime}}\right)
$$

y por lo tanto:

$$
A=\log \left(\frac{1000}{1000-x^{\prime}}\right)=\varepsilon_{\lambda} C_{t} l
$$

Usualmente, el sistema, en el equipo utilizado, trabaja asignando en forma automática el valor de $x_{0}$ correspondiente al voltaje previo al pulso del láser realizando la transformación correspondiente.

\section{Experimentos de fotólisis estacionaria}

Además de los métodos de fotólisis originadas en el empleo de un corto pulso de luz, como se describió anteriormente, la fotólisis estacionaria también es usualmente empleada. La irradiación continua implica la iluminación de la muestra con luz proveniente de una lámpara de intensidad constante y durante largos períodos de tiempo, por lo cual, los experimentos en los que se estudia una reacción fotoquímica inducida por irradiación continua se denominan genéricamente fotólisis continuas ó estacionarias.

Algunos pocos experimentos se han llevado a cabo en este trabajo de tesis utilizando esta modalidad, por lo que se describirá someramente, la manera en que estos se desarrollaron. En 
la figura 5 se muestra un esquema simplicado del dispositivo empleado para la irradiación de las soluciones.

Las concentraciones de los reactivos se ajustaron de modo que más del $99.9 \%$ de la luz incidente fuera absorbida para minimizar la fotólisis secundaria utilizándose, además, buzos magnéticos para agitar las soluciones mientras eran irradiadas. La irradiación continua de la solución se realizó con una lámpara Rayonet Photochemical Reactor Lamp, RPR 3500 A de la Southern N. E. Ultraviolet Co. Esta lámpara emite luz de $350 \mathrm{~nm}$ con un ancho de banda de unos $20 \mathrm{~nm}$.

Figura 5: Esquema del dispositivo empleado para los experimentos de fotólisis estacionaria

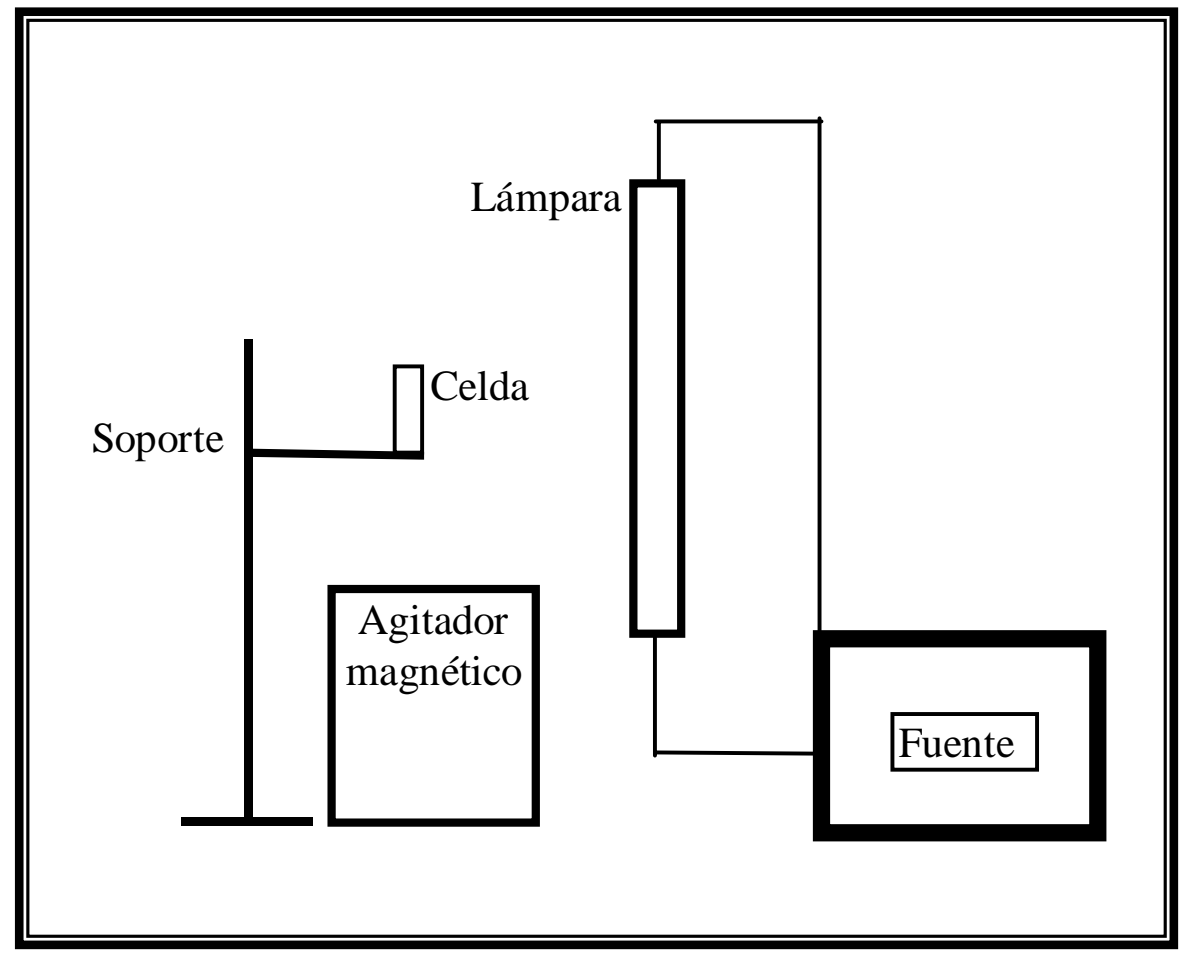

Las soluciones fueron deaereadas con $\mathrm{N}_{2}$ durante 30 minutos antes de cada experimento y el tiempo de irradiación fue medido con un cronómetro de disparo manual a partir del instante 
en que se enciende la lámpara. Los cambios fotoquímicos son monitoreados por intermedio de los espectros de absorción UV-visible ó IR.

Los experimentos de fotólisis estacionaria son una herramienta importante para la determinación del rendimiento cuántico de una reacción fotoquímica determinada. Para ello es necesario conocer la intensidad de emisión de la lámpara empleada para la irradiación. Este último parámetro puede calcularse empleando un actinómetro químico, el cual experimenta, bajo irradiación, una transformación química de rendimiento cuántico conocido. Para nuestros experimentos se utilizó el $\left[\mathrm{Co}\left(\mathrm{NH}_{3}\right)_{5} \mathrm{Br}\right] \mathrm{Br}_{2}$ cuyo rendimiento cuántico a $350 \mathrm{~nm}$ es igual a 0,243 .

\section{Luminiscencia y espectrofluorometría}

\section{i. Emisión en estado estacionario: espectros de emisión, espectros de excitación y} rendimientos cuánticos de emisión

La aplicación satisfactoria de los métodos de luminiscencia requiere una comprensión de la instrumentación utilizada, siendo necesario prestar una atención considerable a los detalles experimentales. Hay dos razones que justifican que esto sea así. Primero, la fluorescencia es un método altamente sensible. La ganancia o amplificación de los instrumentos puede ser incrementada para hacer observables emisiones muy débiles. Sin embargo, estas emisiones pueden no estar originadas por el fluoróforo de interés. Tales interferencias pueden deberse a la emisión del solvente, pérdidas de luz de los instrumentos, luz dispersada por soluciones túrbidas, dispersión Rayleigh y/ó dispersión Raman, para nombrar solo algunas. Segundo, no existe espectrofluorómetro ideal y tales instrumentos no dan verdaderos espectros de excitación ni de emisión debido a la no uniformidad de la salida espectral de las fuentes de luz 
y a la dependencia de la eficiencia de los monocromadores y tubos fotomultiplicadores con la longitud de onda. Además, la polarización o anisotropía de la luz emitida también puede afectar la medición de las intensidades de fluorescencia. Para obtener datos espectrales confiables, entonces, es necesario controlar todos estos factores para poder efectuar las correcciones necesarias.

Un espectro de emisión es la distribución espectral de la emisión de la muestra en estudio, medido excitando con una longitud de onda fija $\left(\lambda_{\text {exc }}\right)$. Inversamente, un espectro de excitación consiste en la dependencia de la intensidad de emisión a una dada longitud de onda $\left(\lambda_{\text {obs }}\right)$, con la variación de la longitud de onda de excitación.

Para un instrumento ideal, el espectro de emisión registrado directamente, representaría el flujo de fotones emitidos a cada longitud de onda, sobre un intervalo de longitud de onda determinado por el ancho de las rendijas y la dispersión del monocromador de emisión. Similarmente, el espectro de excitación representaría el rendimiento cuántico relativo de emisión a cada longitud de onda de excitación. En la figura 6 se muestra un diagrama esquemático del sistema óptico del espectrofluorómetro Perkin-Elmer LS 50B utilizado para nuestros experimentos.

Básicamente, los espectros de emisión en estado estacionario, se obtienen excitando con luz monocromática desde una fuente de excitación luminosa (una lámpara de arco de Xenón en nuestro instrumento), focalizando el haz (cuya pureza espectral se obtiene del monocromador de excitación), sobre la cubeta. Para evitar complicaciones por efecto de la luz dispersada la emisión de la muestra se detecta a $90^{\circ}$ con respecto al haz de excitación. El espectro de emisión se obtiene por barrido de un segundo monocromador (monocromador de emisión) situado entre la muestra y el detector, un tubo fotomultiplicador R928 sensible al rojo. 
Figura 6: Diagrama esquemático del sistema óptico del espectrofluorómetro utilizado para los experimentos de emisión en estado estacionario

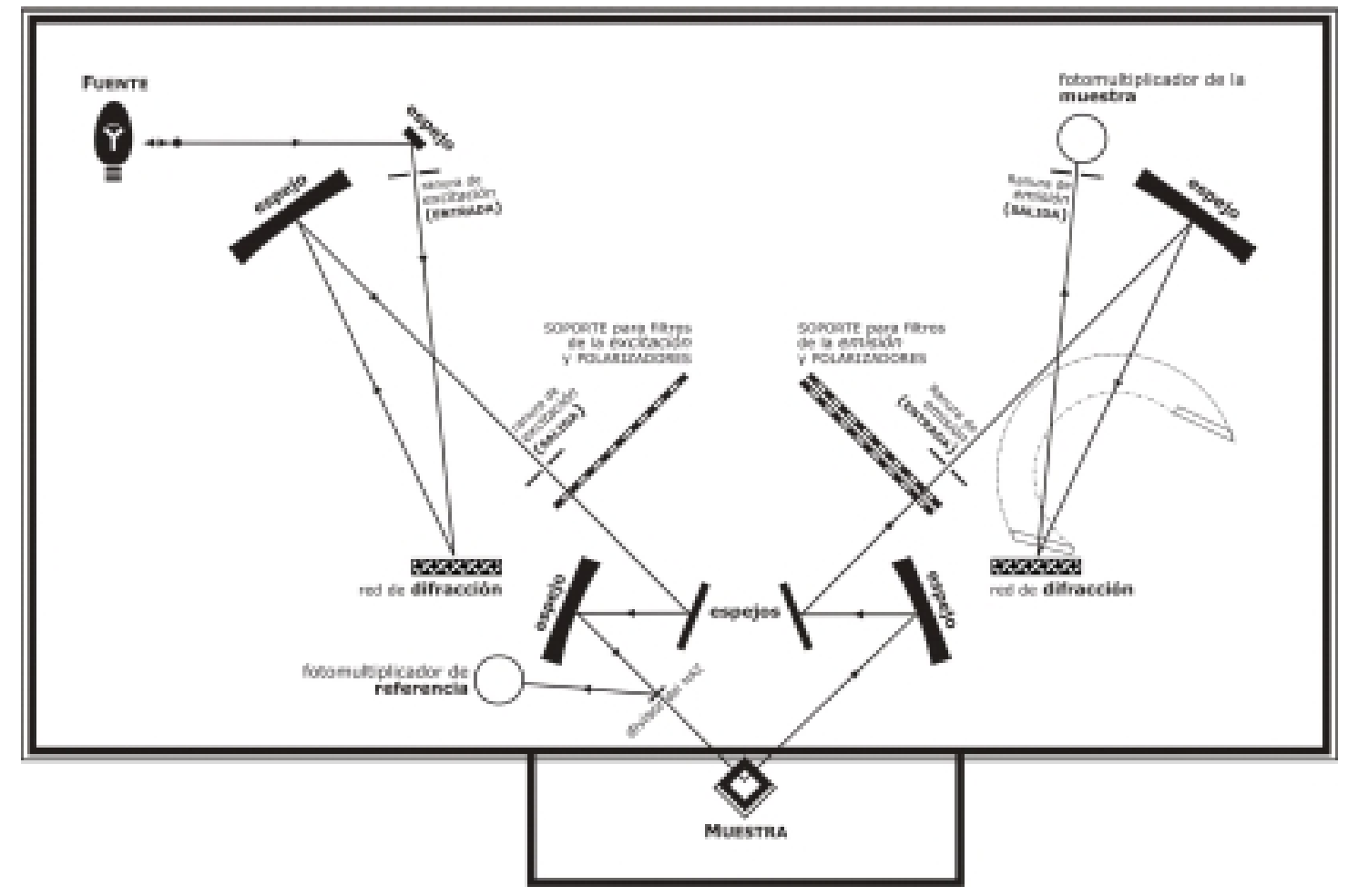

La lámpara de Xenón emite con alta intensidad en todo el rango de longitudes de onda por encima de $250 \mathrm{~nm}$ por lo tanto es muy útil para trabajar en la región UV-visible. Por esta razón se utilizaron celdas de cuarzo de calidad óptica similar a la utilizada en las medidas de láser flash fotólisis. Las soluciones a medir fueron previamente deaereadas con corrientes de $\mathrm{N}_{2}$ libre de $\mathrm{O}_{2}$. El espectro de emisión obtenido es corregido haciendo la diferencia entre éste y el espectro de emisión del solvente ó matriz tomado en las mismas condiciones, y al espectro así obtenido se lo corrige por la respuesta del instrumento (la sensibilidad del detector y la eficiencia de los monocromadores varían con la longitud de onda). Esta corrección se realiza dividiendo la señal de emisión de la muestra por la respuesta del instrumento, que en nuestro caso se obtuvo dividiendo el espectro corregido de una sustancia 
de referencia por el espectro de la misma sustancia tomado en nuestro equipo. El denominado espectro de emisión corregido hace referencia, generalmente, a este tipo de corrección.

Para obtener un espectro de excitación se realiza un barrido con el monocromador de excitación manteniendo fijo el monocromador de emisión, generalmente, a la longitud de onda del máximo de emisión. Los espectros así obtenidos sufren distorsión debido, principalmente, a la dependencia de la intensidad de la luz de excitación con la longitud de onda, es decir, la lámpara no provee una misma intensidad de luz en todo su espectro de emisión. Por lo tanto se debe realizar una corrección de la señal de intensidad de la emisión de la muestra transformándola en una señal proporcional al número de fotones incidentes. En nuestro equipo y mediante el fotomultiplicador de referencia el sistema calibra y corrige automáticamente, de manera que los espectros de excitación corregidos se obtienen en forma directa.

La dependencia con la longitud de onda de la respuesta del monocromador de emisión y del detector que recibe la emisión de la muestra no son importantes debido a que la longitud de onda de emisión se mantiene constante durante el experimento.

Otro factor que hay que tener en cuenta en todos los experimentos de luminiscencia es la posibilidad de que la intensidad aparente de la emisión y su distribución espectral sean dependientes de la densidad óptica de la muestra y de la geometría del sistema. Para la geometría más común usada en espectrofluorometría, que implica la observación de la emisión en ángulo recto de una cubeta iluminada centralmente, se puede demostrar que si la absorbancia de la muestra a la longitud de onda de excitación es de 0,1 la intensidad en el centro de la cubeta, I, es 0,88.Io, donde Io es la intensidad incidente sobre la cubeta. Como la intensidad de la luminiscencia observada es proporcional a la intensidad de la luz de excitación, el rendimiento cuántico aparente será alrededor de un $10 \%$ menor que el observado con una solución infinitamente diluida. Este es el denominado efecto de filtro interno. Tal fenómeno puede disminuir la intensidad de excitación en el punto de observación, 
ó disminuir la emisión observada por absorción de ésta. Por lo general, y para minimizar este efecto, los espectros de emisión y excitación se tomaron utilizando soluciones con una absorbancia a la longitud de onda de excitación, $\mathrm{A}_{\lambda \mathrm{exc}} \leq 0,1$.

Como se mencionó anteriormente, otro parámetro directamente medible en experimentos de emisión en estado estacionario y de vital importancia para la caracterización del estado excitado emisor de un complejo dado, es el rendimiento cuántico de emisión. Los rendimientos cuánticos dan cuenta de la eficiencia de un proceso en términos de la cantidad de energía lumínica absorbida. El rendimiento cuántico de un proceso fotofísico como la emisión de luz, $\phi_{\text {emi, }}$, se puede expresar como el número de moléculas emisoras respecto de los fotones de luz absorbidos:

$$
\phi_{\mathrm{emi}}=\frac{\text { Nro.de moléculas emisoras por unidad de tiempo y por unidad de volumen }}{\text { Nro.de cuantos absorbidos por unidad de tiempo y por unidad de volumen }}
$$

Como el número de fotones absorbidos es proporcional a la intensidad de la radiación absorbida, $I_{A}$, y el número de fotones emitidos es proporcional a la intensidad de la radiación emitida, $I_{e m i}$, se puede considerar y escribir que:

$$
\phi_{\mathrm{emi}}=\frac{\mathrm{I}_{\mathrm{emi}}}{\mathrm{I}_{\mathrm{A}}}
$$

Nuevamente debemos hacer la siguiente consideración: la expresión anterior es estrictamente válida cuando el estado excitado emisor es poblado en forma directa por la absorción de luz, es decir, cuando el rendimiento cuántico coincide con la eficiencia $\eta$. Si, por otro lado, el estado excitado emisor es poblado en forma indirecta siguiendo algún camino no radiativo, desde otro estado excitado que absorbió la luz, entonces el $\phi_{\text {emi }}$ se obtiene multiplicando la 
expresión por la eficiencia del camino seguido. Como se mencionó anteriormente, éste es el caso para los estados excitados ${ }^{3}$ TCML de los complejos en estudio que son poblados desde el correspondiente singlete a través de un paso de cruzamiento intersistemas. Por lo tanto, la expresión adecuada será:

$$
\phi_{\mathrm{emi}}=\eta_{\mathrm{isc}} \frac{\mathrm{I}_{\mathrm{emi}}}{\mathrm{I}_{\mathrm{A}}}
$$

pero como $\eta_{\text {isc }} \sim 1$ la ecuación anterior se vuelve a transformar en la expresión original.

Experimentalmente, los rendimientos cuánticos de emisión de la solución muestra en estudio, se determinan por comparación de la intensidad de la emisión, $I_{e m i}$, tomada como el área bajo la curva del espectro de emisión, $I_{\text {muestra, }}$ y la intensidad de emisión, tomado en iguales condiciones, de una solución de una referencia de rendimiento cuántico conocido, $I_{\text {referencia }}$ La referencia a utilizar debería ser ópticamente comparable con la muestra (es decir, tener la misma densidad óptica y camino óptico y similar perfil de energía de emisión). Si la absorbancia de ambas soluciones a la longitud de onda de excitación, $A_{\text {muestra }}$ y $A_{\text {referencia }}$, es diferente, debe realizarse una corrección teniendo en cuenta esta condición. Finalmente, y tomando ambos espectros con el mismo equipo y en idénticas condiciones de temperatura, celda utilizada, abertura de las rendijas, etc., los rendimiento cuánticos de emisión relativo fueron calculados de acuerdo a la siguiente ecuación:

$$
\phi_{\mathrm{emi}}=\left(\frac{\mathrm{A}_{\text {referencia }}}{\mathrm{A}_{\text {muestra }}}\right)\left(\frac{\mathrm{I}_{\text {muestra }}}{\mathrm{I}_{\text {referencia }}}\right) \phi_{\text {emi, referencia }}\left(\frac{\mathrm{n}_{\text {muestra }}}{\mathrm{n}_{\text {referencia }}}\right)^{2}
$$

donde $n$ es el índice de refracción del solvente puro empleado en cada una de las soluciones. Estas medidas requieren, como mencionamos más arriba, que las soluciones sean ópticamente 
diluidas $\left(\mathrm{A}_{\lambda_{\mathrm{exc}}} \square 0,1\right)$ permitiendo, además, despreciar las diferencias entre los $n$ del solvente puro y la solución.

\section{ii. Estimación de las energías de punto 0-0 $\left(E_{00}\right)$}

Por absorción de luz de energía apropiada, se puede poblar un estado excitado de transferencia de carga del metal hacia el ligando. Si el estado fundamental es de naturaleza singlete, entonces el estado poblado será ${ }^{1}$ TCML. La superficie de energía potencial de este estado puede estar levemente desplazada con respecto a la del estado fundamental y por lo tanto la transición se puede producir hacia algún nivel vibracional más alto con respecto al $v=$ 0 de este estado. La energía que caracteriza este proceso está dada por la frecuencia $v$ en el máximo de absorción de la banda de transferencia de carga, $v_{\text {máx }}$, considerando que las moléculas absorbentes se encuentran en el nivel vibracional cero del estado fundamental. Las que absorben desde niveles más altos pueden caracterizarse determinando la energía correspondiente al final de esta banda de absorción, es decir, cuando la cola de la banda de absorción decrece asintóticamente a cero, siendo $v_{\text {th }}$. Luego de la absorción se produce un reacomodamiento de la energía como resultado de la cual todas las moléculas excitadas se encuentran en el nivel vibracional cero. A esta energía se la denomina energía de reorganización, $\lambda_{\text {abs. }}$.

La diferencia de energía entre el estado fundamental y el estado excitado cuando ambos estados son tomados en sus niveles vibracionales cero es la energía del punto $0-0,{ }^{1} \mathrm{E}_{00}$ para este estado ${ }^{1}$ TCML. Esta energía puede considerarse equivalente a la necesaria para oxidar al $\operatorname{Re}^{\mathrm{I}}$ a $\operatorname{Re}^{\mathrm{II}}$ y reducir al ligando $\mathrm{L}_{\text {a }} \mathrm{L}^{-}$. Así, la diferencia entre los potenciales electroquímicos de media onda, $\mathrm{E}_{1 / 2} \mathrm{Re}^{\mathrm{I} / \mathrm{II}}$ y $\mathrm{E}_{1 / 2} \mathrm{~L} / \mathrm{L}$, puede considerarse igual a ${ }^{1} \mathrm{E}_{00}$. 
Pasar desde el estado ${ }^{1}$ TCML al ${ }^{3}$ TCML implica una cantidad de energía representada como $\Delta \mathrm{ST}$ ó diferencia de energía singlete-triplete. Este valor se puede estimar de la diferencia de energía entre ${ }^{1} \mathrm{E}_{00}$ y ${ }^{3} \mathrm{E}_{00}$. Para ello, la energía ${ }^{3} \mathrm{E}_{00}$ puede ser estimada de los espectros de emisión definiendo términos equivalentes a los definidos para la absorción, $v_{\max }^{\mathrm{e}}, v_{\mathrm{th}} \mathrm{y} \lambda_{\mathrm{em}}$. En el siguiente esquema se representan, para mayor claridad, los términos descriptos:

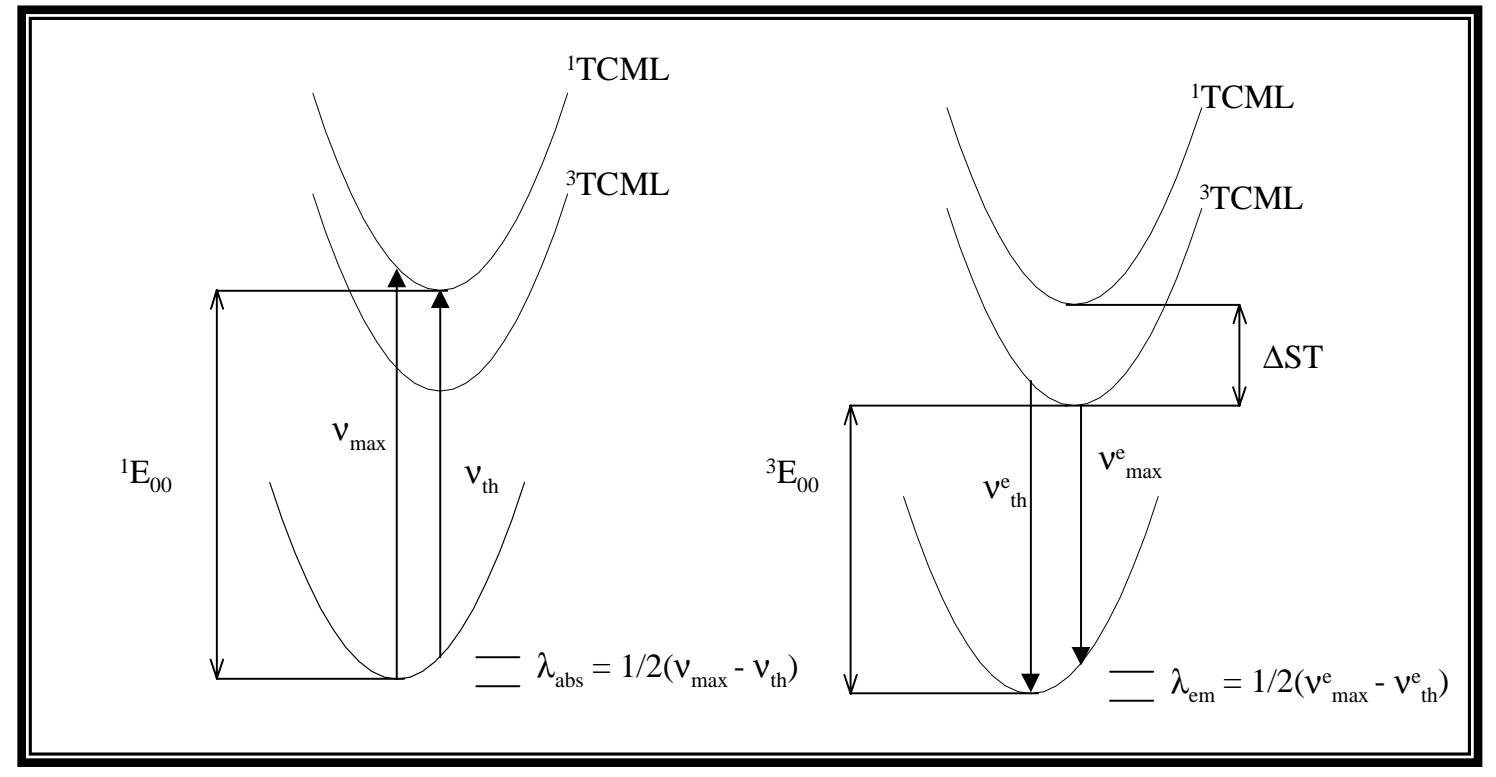

De los espectros de absorción y emisión y de las medidas electroquímicas se pueden estimar los $\mathrm{E}_{00}$ correspondientes utilizando las siguientes relaciones:

$$
\begin{gathered}
\mathrm{E}_{1 / 2} \mathrm{Re}^{\mathrm{I} / \mathrm{II}}-\mathrm{E}_{1 / 2} \mathrm{~L} / \mathrm{L}^{-}={ }^{1} \mathrm{E}_{00}=v_{\text {máx }}-\lambda_{\text {abs }} \\
{ }^{1} \mathrm{E}_{00}-{ }^{3} \mathrm{E}_{00}=\Delta \mathrm{ST} \\
{ }^{3} \mathrm{E}_{00}=v_{\text {max }}^{\mathrm{e}}+\lambda_{\text {em }}
\end{gathered}
$$




\section{iii. Emisión resuelta en el tiempo}

Mientras que de las medidas de emisión en estado estacionario se obtiene información sobre los rasgos espectrales de la emisión (es decir, máximo de emisión, intensidad integrada, dependencia con la temperatura ó el solvente), de los experimentos de emisión resuelta en el tiempo se adquiere información cinética a una longitud de onda, normalmente la correspondiente al máximo de emisión.

Existen distintos métodos con los cuales es posible realizar medidas de la emisión resuelta en el tiempo que incluye a los de modulación de fase, los de excitación pulsada y los de conteo de fotones (single photon counting). La mayoría de las medidas realizadas en este trabajo de tesis se llevaron a cabo utilizando métodos pulsados cuya fuente de excitación fue un láser de nitrógeno, en un sistema similar al descripto para láser flash fotólisis (ver figura 3). Algunos experimentos se llevaron a cabo excitando con un láser de excímero. La diferencia fundamental con respecto a las determinaciones de $\Delta \mathrm{DO}$ originadas en la presencia de la especie transitoria, es que no se requiere el uso de la luz de análisis.

Para obtener información cinética de una medida del decaimiento de la traza de emisión, es necesario que el ancho del pulso de excitación sea estable y reproducible, más corto que el tiempo de vida del estado excitado en estudio. Además, debe ser de energía suficiente como para generar una cantidad detectable de fotones emitidos, pero no tan elevada como para inducir eventos multifotónicos. También, la velocidad en la repetición del pulso de excitación debe ser lo suficientemente baja como para permitir un completo retorno, entre pulsos, al estado fundamental. La solución muestra, previamente deaereada, se coloca en una celda de cuarzo de calidad óptica. El detector está ubicado a $90^{\circ}$ del haz de excitación y en el monocromador se selecciona la $\lambda_{\text {obs. }}$ La señal de salida es promediada convenientemente con el fin de obtener una buena relación señal / ruido. 
La mayoría de las medidas de los tiempos de vida de un estado excitado involucra procesos de primer orden ó de pseudo-primer orden. Un proceso de estas características puede describirse con la siguiente ecuación:

$$
\frac{\left.\mathrm{d}^{[*} \mathrm{D}\right]}{\mathrm{dt}}=-\mathrm{k}\left[{ }^{*} \mathrm{D}\right]
$$

donde ${ }^{*} D$ representa al estado excitado, $\mathrm{d}\left[{ }^{*} D\right] / \mathrm{dt}$ su velocidad de desaparición y $k$ es la constante de velocidad específica de primer orden en $\mathrm{s}^{-1}$ para la desaparición de * $D$.

Cuando se monitorea la luminiscencia de ${ }^{*} D$, la señal de emisión es frecuentemente proporcional a la concentración de ${ }^{*} D,\left[{ }^{*} D\right]$, es decir,

$$
\text { velocidad de emisión de fotones }=\mathrm{k}_{\mathrm{r}}\left[{ }^{*} \mathrm{D}\right]
$$

donde ahora $k_{r}$ es la constante de velocidad específica de emisión ó radiativa. La resolución de esta ecuación permite obtener la siguiente expresión:

$$
\left[{ }^{*} \mathrm{D}\right]=\left[{ }^{*} \mathrm{D}\right]_{0} \mathrm{e}^{-\mathrm{k} \mathrm{t}}
$$

donde $\left[{ }^{*} D\right]_{0}$ es la $\left[{ }^{*} D\right]$ inicial. En función de esta última expresión se espera que los decaimientos radiativos sean de tipo exponencial.

El tiempo que debe transcurrir para que el número de moléculas excitadas disminuya desde ${ }^{*} D_{0}$ hasta ${ }^{*} D_{0} / 2$, se denomina tiempo de vida media ó $\mathrm{t}_{1 / 2}$. En medidas de emisión, será el tiempo necesario para que la intensidad de emisión decaiga a la mitad. A pesar de que el $\mathrm{t}_{1 / 2}$ se puede determinar fácilmente desde una traza del osciloscopio, este parámetro tiene una significancia teórica limitada. Una cantidad fundamental y de mayor significancia, es el 
tiempo de vida promedio en el cual el número de moléculas excitadas disminuye desde ${ }^{*} D_{0}$ hasta ${ }^{*} D_{0} / e$. A este parámetro se lo denomina usualmente como $\tau$. Así, cuando la intensidad de la emisión alcance un valor de 1/e de su valor inicial, el tiempo de vida de la luminiscencia será el $\tau_{\text {emi }}$ y por lo tanto, en una situación donde el radiativo sea el único camino de desactivación, se puede establecer que,

$$
\tau_{\mathrm{emi}}=\frac{1}{k_{r}}
$$

Por último, es importante mencionar que frecuentemente el $\tau_{\mathrm{emi}}$ calculado en experimentos de emisión resuelta en el tiempo es idéntico al obtenido para el decaimiento de las trazas en experimentos de láser flash fotólisis con monitoreo de espectroscopía de absorción. Esto se puede racionalizar considerando que es consecuencia de la observación del mismo estado excitado pero en dos experimentos diferentes.

\section{Electroquímica}

\section{i. Voltametría cíclica}

Voltametría cíclica es una técnica no estacionaria, que consiste en un barrido de potencial y registro de la corriente, utilizada ampliamente en sistemas electroquímicos diversos para obtener información de parámetros cinéticos y, en casos especiales, termodinámicos.

En nuestro caso, esta técnica se utilizó trabajando a bajas velocidades de barrido, con el objeto de determinar los potenciales redox de los complejos y de los ligandos sintetizados, para su caracterización. Además, estos potenciales fueron luego utilizados para estimar la energía involucrada en un proceso de transferencia intramolecular de un electrón desde el centro metálico hacia un ligando. 
La voltametría cíclica convencional de continua, implica la aplicación de un barrido lineal de potencial entre el electrodo de trabajo y un contraelectrodo. Para ello se utilizó una celda electroquímica compuesta por un electrodo de trabajo (disco de Pt pulido a espejo en nuestro sistema), un contraelectrodo (malla de $\mathrm{Pt})$, un electrodo de referencia $\left(\mathrm{Ag} / \mathrm{AgCl} / \mathrm{KCl}_{\text {sat }}\right)$ y un electrolito soporte (solución $\geq 0,1 \mathrm{M}$ de hexafluorofosfato de tetrabutilamonio). Las medidas se llevaron a cabo con soluciones de la muestra en acetonitrilo (secado en óxido básico activo de aluminio 90 durante varios días) que habían sido previamente desoxigenadas durante más de 30 minutos con $\mathrm{N}_{2}$.

Para la mayoría de nuestras determinaciones se utilizó una variante de esta técnica que es la voltametría cíclica de alterna en donde el potencial del electrodo de trabajo es modulado a una cierta frecuencia con una onda de forma sinusoidal de $10 \mathrm{mV}$ de amplitud pico a pico. Estas condiciones de trabajo permiten una mayor sensibilidad para la determinación de la corriente. Además, la medida de los datos es mucho más sencilla ya que, cuando un proceso redox ocurre, las curvas tienen forma de picos de ondas de corriente. Luego de cada voltamperograma, se procedió al pulido a espejo del electrodo de trabajo para eliminar la posible formación de films adheridos a la superficie del mismo.

\section{ii. Espectroelectroquímica}

En los últimos años el estudio de los procesos de quenching del estado excitado se ha ido complementando con el uso de otras técnicas. La espectroelectroquímica es una de ellas ya que nos permite obtener los espectros de absorción UV-visible de las especies reducidas generadas in-situ, modulando el potencial del electrodo de trabajo alrededor de las cuplas de interés. El equipo utilizado para nuestras determinaciones es un espectrómetro computarizado de reflectancia externa especular específico para el estudio, in-situ, de la interface 
electrodo/solución. En este método, la radiación incide con un ángulo determinado sobre el electrodo (de superficie espejada) desde la solución y se refleja en el mismo hacia el sistema detector. Con esta técnica, es posible detectar fracciones de monocapa adsorbidas sobre metales, determinar perfiles de concentración en solución por absorción en la interface a una dada longitud de onda, detectar diferentes intermediarios de reacción a través de su diferente respuesta espectral, etc. Para el estudio de intermediarios electrogenerados de vida corta, esta técnica, de alta sensibilidad, permite una resolución temporal de $\sim 1 \mathrm{~ms}$.

El equipo utilizado en las medidas de reflectancia en sistemas electroquímicos se representa, en forma esquemática, en la figura 7.

La radiación policromática de una lámpara de cuarzo-halógeno de $250 \mathrm{~W}$, alimentada por una fuente regulada de tensión continua, se enfoca a través de lentes condensadores en la rendija de entrada de un monocromador doble. Entre la lámpara y el monocromador, en algunos casos, se intercala un modulador de luz (“chopper”) para determinar la intensidad de la radiación. La luz monocromática emergente puede ser linealmente polarizada paralela (\|, p) ó perpendicular $(\perp, s)$ al plano de incidencia de la superficie especular del electrodo por medio de un prisma de Glan-Taylor de alta transmisión en la zona UV-visible. En nuestros experimentos, no es necesario el uso de luz polarizada. El uso de filtros y lentes permite, respectivamente, eliminar órdenes superiores de difracción y el enfoque del haz luminoso. El ángulo de incidencia, para determinar la contribución a la reflectancia total del efecto producido por la absorción de las especies reducidas en la interface, es de $45^{\circ}$. Por lo tanto, la celda óptica utilizada, posee ventanas de cuarzo con la orientación adecuada a este ángulo de incidencia. En el tipo de medidas más común, el potencial del electrodo de trabajo se modula con un potencial alterno de onda cuadrada (de amplitud $\geq$ al ancho de la base de la onda redox) sobreimpuesto a un potencial de continua, a través de un potenciostato / generador de funciones. Teniendo en cuenta el potencial en que ocurre un proceso de reducción, $\mathrm{E}_{\mathrm{r}}$, se 
puede considerar que si la modulación se realiza entre un potencial más positivo que $\mathrm{E}_{\mathrm{r}}$ (donde la especie reducida no está presente) y un potencial más negativo que $\mathrm{E}_{\mathrm{r}}$ (donde la especie reducida ya ha sido generada), entonces, los cambios pequeños en la absorción de la interface se traducen en cambios en la intensidad de la luz reflejada que contiene la información del proceso en estudio. Es decir, estos cambios son producto de la diferente absorción de la especie reducida con respecto a la especie sin reducir.

Estas variaciones en la intensidad de la luz reflejada son detectadas por un fotomultiplicador que posee un sistema electrónico de realimentación que mantiene una corriente constante de $60 \mu \mathrm{A}$ y que equivale a normalizar los cambios en la intensidad modulada con respecto a las variaciones de intensidad de la radiación provenientes de la curva espectral de la lámpara, la función de transferencia óptica del sistema, etc., durante un barrido de longitud de onda. Los cambios en la reflectancia del electrodo, $\mathrm{R}$, definida como el cociente entre la intensidad reflejada y la intensidad incidente, $\mathrm{I}_{\mathrm{r}} / \mathrm{I}_{\mathrm{i}}$, son demodulados por un amplificador de detección sincrónico ("lock-in”) siendo el espectro final la diferencia normalizada, $\Delta \mathrm{R} / \mathrm{R}$, correspondiente a la reflectancia a los potenciales de la modulación dividido por el valor normalizador constante R.

Alternativamente, se trabajó con un equipo provisto de un analizador multicanal óptico (OMA) controlado por microprocesador, que permite la adquisición de espectros a un potencial constante ó mediante un barrido lento de potencial, es decir, sin la necesidad de modular, utilizando un salto de potencial, el electrodo de trabajo. De esta manera típicamente se toma un espectro cada $50 \mathrm{mV}$ para un barrido de potencial determinado entre ciertos límites, obteniendo una secuencia rápida de espectros correspondiente a la reflectancia absoluta a cada potencial. Posteriormente, con programas computacionales adecuados, se realiza la normalización obteniendo los espectros de $\Delta \mathrm{R} / \mathrm{R}$ de la siguiente manera: si se toma como $\mathrm{R}$ a la reflectancia absoluta a un potencial determinado y $\mathrm{R}_{\mathrm{ref}}$ a la reflectancia, a otro 
potencial, elegida como referencia, entonces, $\Delta R / R_{\text {ref }}=\left(R-R_{\text {ref }}\right) / R_{\text {ref }}$ es la diferencia normalizada de la reflectancia. En general, se toma como $\mathrm{R}_{\text {ref }}$ a la señal obtenida a un potencial donde la especie reducida no está presente.

Además, se considera en todas las medidas de espectroelectroquímica, que se cumple con la ecuación simple para la absorción de luz en la capa difusional, $\ln (1+\Delta \mathrm{R} / \mathrm{R})=-\Delta \mathrm{A}$, en donde el electrodo metálico se considera como reflector perfecto y $\Delta \mathrm{A}$ son los cambios de la absorción. De esta expresión se puede obtener la ecuación aproximada $\Delta \mathrm{R} / \mathrm{R} \cong-\Delta \mathrm{A}$. Por lo tanto los cambios normalizados en la reflectancia pueden ser considerados como directamente proporcionales a los cambios en la absorbancia.

Figura 7: Esquema del equipo de reflectancia externa especular (espectroelectroquímica) UV-visible. CE: contraelectrodo, ER: electrodo de referencia y ET: electrodo de trabajo

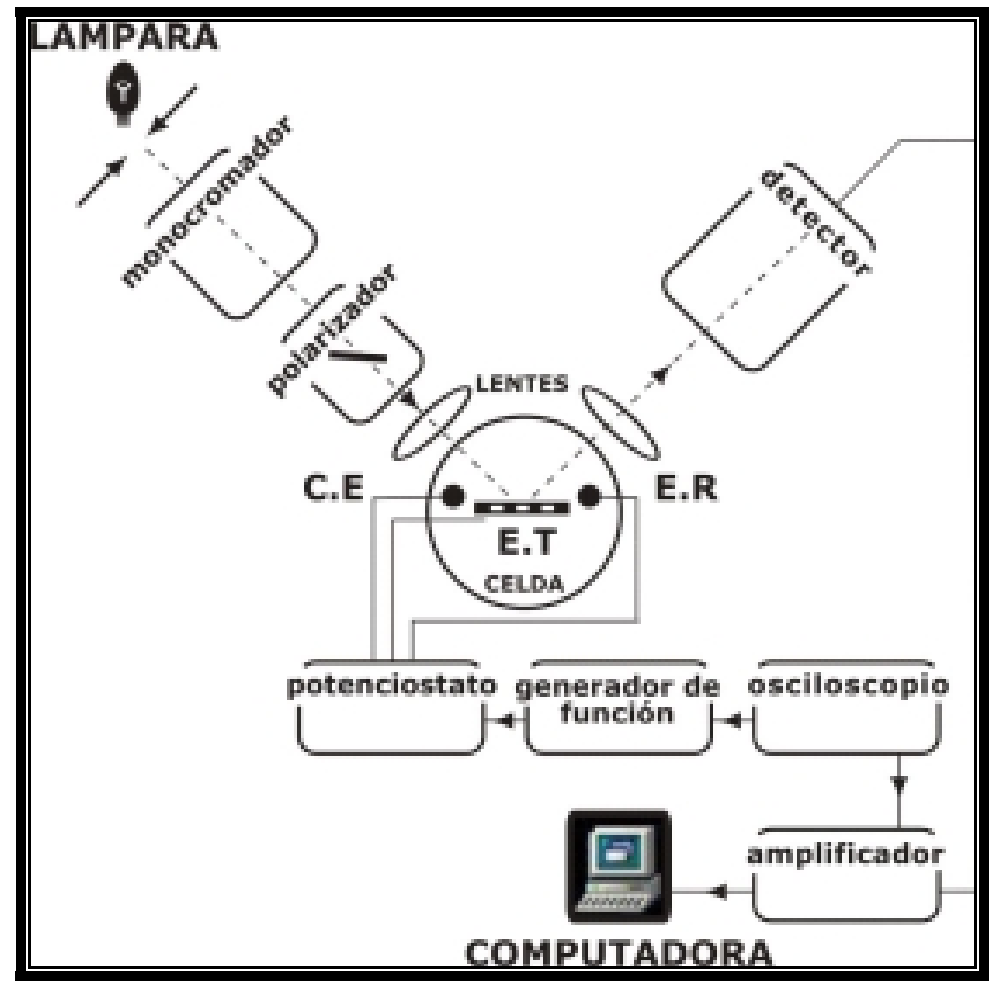


Todas las determinaciones realizadas se llevaron a cabo realizando previamente, y en las condiciones descriptas anteriormente, medidas de voltametría cíclica para confirmar la ausencia de impurezas y definir las zonas de potencial de trabajo

La configuración experimental para la obtención de espectros de absorción IR in-situ de las especies reducidas es, básicamente, similar al descripto para los estudios UV-visible, excepto por el reemplazo de elementos de vidrio óptico por otros de cuarzo, silicio ó de algún otro material transparente al IR (ventana de $\mathrm{CaF}_{2}$ en nuestro caso) y, fundamentalmente, por el cambio en el tipo de la fuente de radiación y detector. Estos, deben ser adecuados para emitir y detectar, respectivamente, en la región del infrarrojo medio. En general, los cambios en la reflectancia IR son muy pequeños y por lo tanto es necesario promediar la señal obtenida luego de la modulación del potencial. Otros detalles experimentales son mencionados en el capítulo 4.

Todos las medidas electroquímicas realizadas para el presente trabajo de tesis se llevaron a cabo en el laboratorio que el Dr. R.O. Lezna posee en el INIFTA. 
Bibliografía consultada para la Parte I del presente trabajo de tesis

[1] Bates W.D., Trabajo de tesis doctoral, University of North Carolina at Chapel Hill, 1994

[2] Wolcan E., Trabajo de tesis doctoral, Universidad Nacional de La Plata, 1996

[3] Hoffman, M.Z., “Inorganic Photochemistry--State of the Art”. J. Chem. Educ. 1983, Vol 60, Nro.10, 784-887

[4] Julliard M. and Chanon M. Chem. Rev. 1983, 83, 425-506

[5] Joseph R. Lakowicz “Principles of Fluorescence Spectroscopy”, Plenum Press Ed., New York 1983.

[6] Ferraudi, G.J., “Elements of Inorganic Photochemistry” Ed. John Wiley \& Sons, New York 1988.

[7] Scaiano J.C., “Handbook of Organic Photochemistry” Vol. 1, CRC Press, Boca Raton, 1989.

[8] Kalyanasundaram K. and Grätzel M. “Photosensitization and Photocatalysis Using Inorganic and Organometallic Compounds” Kluwer Academic Publishers, Dordrecht, 1993. [9] Stufkens D.J. and Vlpek Jr A. Coord. Chem. Rev. 177 (1998) 127-179.

[10] Fox M.A. and Chanon M. “Photoinduced Electron Transfer. Part B: Experimental Techniques and Medium Effects” Elsevier Science Publishers B.V., Amsterdam, 1988.

[11] Turro N., “Molecular Photochemistry”, W.A. Benjamin, Inc. 1965.

[12] Demas, J.N., “Excited State Lifetime Measurements”; Academic Press: New York 1983.

[13] R.O. Lezna, An. Asoc. Quím. Argent. 76(1), 25 (1988).

[14] R.O. Lezna, An. Asoc. Quím. Argent. 82(4), 293 (1994).

[15] Southampton Electrochemistry Group, “Instrumental Methods in Electrochemistry” Ellis Horwood Limited, Chichester, 1985. 
PARTE II

\section{SINTESIS, ESTUDIOS FOTOQUÍMICOS, FOTOFÍSICOS Y FOTOELECTROQUÍMICOS DE UN COMPLEJO DE Re ${ }^{\mathrm{I}}$ DEL TIPO $f a c-\left[\mathrm{L}-\mathrm{COO}-\mathrm{Re}^{\mathrm{I}}(\mathrm{CO})_{3}\left(2,2^{\prime}\right.\right.$ 'bipiridina $\left.)\right]$}




\section{Introducción}

La sustitución de un ligando haluro X por un "ligando espectador" $\mathrm{L}_{\mathrm{S}}$ en complejos del tipo $\left[\operatorname{Re}^{\mathrm{I}} \mathrm{XL}_{\mathrm{A}}(\mathrm{CO})_{3}\right]$, donde $\mathrm{L}_{\mathrm{A}}$ es un bis(acina monodentada) o una acina bidentada ${ }^{1)}$, ha provisto procedimientos útiles para la preparación de complejos relacionados $\left[\operatorname{Re}^{\mathrm{I}} \mathrm{L}_{S} \mathrm{~L}_{\mathrm{A}}(\mathrm{CO})_{3}\right]$ [1]. El interés en las propiedades que impone $\mathrm{L}_{\mathrm{S}}$ sobre la estructura $-\operatorname{Re}^{\mathrm{I}} \mathrm{L}_{\mathrm{A}}(\mathrm{CO})_{3}$ está relacionado con las posibles aplicaciones que los complejos de $\mathrm{Re}^{\mathrm{I}}$ tendrían en procesos bioquímicos [2] y en la reducción de $\mathrm{CO}_{2}$ [3]. También, la derivatización de polímeros con cromóforos como el grupo $-\operatorname{Re}^{\mathrm{I}} \mathrm{L}_{\mathrm{A}}(\mathrm{CO})_{3}$ [4], ha incentivado las nivestigaciones sobre el rol de $\mathrm{L}_{S}$ en la reactividad de algunos monómeros de $\operatorname{Re}^{\mathrm{I}}$ [5]. En la literatura se ha mostrado, por ejemplo, que la función de $\mathrm{L}_{\mathrm{S}}$ es ejercer pequeñas perturbaciones que alteran la estructura electrónica del cromóforo y modifican su fotofísica [6]. Por otro lado, si existe una fuerte interacción electrónica entre $\mathrm{L}_{\mathrm{s}}$ y el cromóforo de $\mathrm{Re}^{\mathrm{I}}$, el ligando espectador puede participar en procesos redox similares a aquellos en los que está involucrado $\mathrm{L}_{\mathrm{A}}$. En efecto, ha sido observada la transferencia de electrones a $\mathrm{L}_{\mathrm{A}}$ y a $\mathrm{L}_{\mathrm{S}}$ en el $\left[\operatorname{Re}^{\mathrm{I}}(4\right.$-nitrobenzoato $\left.) \mathrm{L}_{\mathrm{A}}(\mathrm{CO})_{3}\right]\left(\mathrm{L}_{\mathrm{A}}=1,10\right.$ fenantrolina, 2,2'bipiridina y (4-fenilpiridina) 2 ), compitiendo los estados excitados entre ellos ó entre los radicales monoreducidos en estado fundamental. Por ejemplo, la reducción de los complejos por radicales ${ }^{\bullet} \mathrm{CH}_{2} \mathrm{OH}$ es seguida de un equilibrio entre especies de $\mathrm{Re}^{\mathrm{I}}$ con ligandos-radicales coordinados, ecuaciones (1) y (2).

$$
\begin{aligned}
{\left[\operatorname{Re}^{\mathrm{I}}\left(4-\mathrm{O}_{2} \mathrm{~N}-\mathrm{C}_{6} \mathrm{H}_{4}-\mathrm{CO}_{2}\right) \mathrm{L}_{\mathrm{A}}(\mathrm{CO})_{3}\right]+{ }^{\circ} \mathrm{CH}_{2} \mathrm{OH} \longrightarrow } & {\left[\operatorname{Re}^{\mathrm{I}}\left(4-\mathrm{O}_{2} \mathrm{~N}-\mathrm{C}_{6} \mathrm{H}_{4}-\mathrm{CO}_{2}{ }^{\bullet} \mathrm{L}_{\mathrm{A}}(\mathrm{CO})_{3}\right]^{-}+\right.} \\
& +\mathrm{CH}_{2} \mathrm{O}+\mathrm{H}^{+}
\end{aligned}
$$

\footnotetext{
1) Abreviaturas usadas en esta parte del presente trabajo de tesis: 2,2'-bipiridina, 2,2'bipi; 2,2'biquinolina, 2,2'biq; piracina, pna; Trietanolamina, TEOA; Trietilamina, TEA; 4-Nitrobenzoato, $\mathrm{NO}_{2} \mathrm{Bz}$; Piracina-2carboxilato, pna-2- $\mathrm{CO}_{2}$; Antraquinona-2-carboxilato, aq-2- $\mathrm{CO}_{2}$; ácido Antraquinona-2- $\mathrm{COOH}$, aq-2- $\mathrm{CO}_{2} \mathrm{H}$; Antraquinona-2-carboxilato de $\mathrm{Ag}$, aq-2- $\mathrm{CO}_{2} \mathrm{Ag}$; diclorometano, DCM; acetonitrilo, $\mathrm{ACN}$; éter etílico, $\mathrm{Et}_{2} \mathrm{O}$; metanol, $\mathrm{MeOH}$; estado excitado de transferencia de carga $\mathrm{Re}$ a $\mathrm{X}, \mathrm{TCML}_{\mathrm{Re} \rightarrow \mathrm{X}}$; estado excitado intraligando, IL.
} 


$$
\left[\operatorname{Re}^{\mathrm{I}}\left(4-\mathrm{O}_{2} \mathrm{~N}-\mathrm{C}_{6} \mathrm{H}_{4}-\mathrm{CO}_{2}{ }^{\bullet}\right) \mathrm{L}_{\mathrm{A}}(\mathrm{CO})_{3}\right]^{-} \rightleftarrows\left[\operatorname{Re}^{\mathrm{I}}\left(4-\mathrm{O}_{2} \mathrm{~N}-\mathrm{C}_{6} \mathrm{H}_{4}-\mathrm{CO}_{2}\right) \mathrm{L}_{\mathrm{A}}{ }^{\bullet}(\mathrm{CO})_{3}\right]^{-}
$$

En contraste con las reacciones en estado fundamental, un estado excitado de transferencia de carga $\mathrm{Re}^{\mathrm{I}}$ a (4-Nitrobenzoato), $\mathrm{TCML}_{\mathrm{Re} \rightarrow \mathrm{NO} 2 \mathrm{Bz}}$, es producido por un mecanismo fotoquímico complejo, ecuaciones (3)-(5): la fotogeneración de un estado excitado de transferencia de

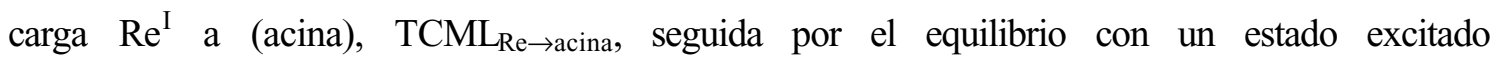
intraligante, IL, precede a la formación del $\mathrm{TCML}_{\mathrm{Re} \rightarrow \mathrm{NO} 2 \mathrm{Bz}}$.

$$
\begin{aligned}
& {\left[\operatorname{Re}^{\mathrm{I}}\left(4-\mathrm{O}_{2} \mathrm{~N}-\mathrm{C}_{6} \mathrm{H}_{4}-\mathrm{CO}_{2}\right)(\mathrm{acina})(\mathrm{CO})_{3}\right]+h \boldsymbol{v} \longrightarrow \mathrm{TCML}_{\mathrm{Re} \rightarrow \text { acina }}} \\
& \mathrm{TCML}_{\mathrm{Re} \rightarrow \text { acina }} \longleftrightarrow \mathrm{IL} \\
& \mathrm{IL} \longrightarrow \mathrm{TCML}_{\mathrm{Re} \rightarrow \mathrm{NO} 2 \mathrm{Bz}}
\end{aligned}
$$

Las propiedades fotoquímicas y fotofísicas del $\left(\mathrm{NO}_{2} \mathrm{Bz}\right) \mathrm{Re}^{\mathrm{I}} \mathrm{L}_{\mathrm{A}}(\mathrm{CO})_{3}$ [7] y del piracina-2- $\mathrm{CO}_{2}$ $\operatorname{Re}^{\mathrm{I}}(\mathrm{CO})_{3}\left(2,2^{\prime}\right.$ bipi) [8] han sugerido fuertemente que el grupo carboxilato aísla electrónicamente al cromóforo de $\mathrm{Re}^{\mathrm{I}}$ de los grupos 4 nitrofenil y piracina unidos a través del grupo $-\mathrm{CO}_{2-}$.

En el primer capítulo de esta parte del presente trabajo de tesis, se describe la preparación y caracterización de un nuevo complejo de esta serie, el Antraquinona-2-COO$\operatorname{Re}^{\mathrm{I}}(\mathrm{CO})_{3}\left(2,2^{\prime}\right.$ bipiridina $)$.

A continuación, el estudio de la fotofísica y reactividad redox de éste y del ligando Antraquinona-2-COOH, para saber cómo afecta la aislación producida por el grupo 
carboxilato del ligando, a las fotoreaciones iniciadas en los estados excitados de transferencia de carga TCML $\operatorname{Re}^{\mathrm{I}}$ a $\mathrm{L}_{\mathrm{A}}$ y $\operatorname{Re}^{\mathrm{I}}$ a $\mathrm{L}_{S}$ y estados excitados intraligantes IL.

Por último, se presentan estudios de espectroelectroquímica UV-visible / IR, con el objetivo de establecer comparaciones entre las especies generadas electroquímicamente y las formadas por reducción fotoquímica de los estados excitados, del complejo de $\mathrm{Re}^{\mathrm{I}}$ y ligandos, en presencia de donores de electrones. Además, observando en la región IR correspondiente a las vibraciones de los carbonilos, se explora la distribución de carga dentro del complejo en respuesta a la inyección de carga en las diferentes estructuras orgánicas (pertenecientes a los ligandos) que lo constituyen, ya que se espera se produzcan, por efecto de la retrodonación, modificaciones en la frecuencia de estiramiento de los $\mathrm{C} \equiv \mathrm{O}[9]$.

Los estructuras de los ligandos aq- $\mathrm{CO}_{2} \mathrm{H}$ y 2,2'bipiridina se muestran a continuación

2,2'bipiridina

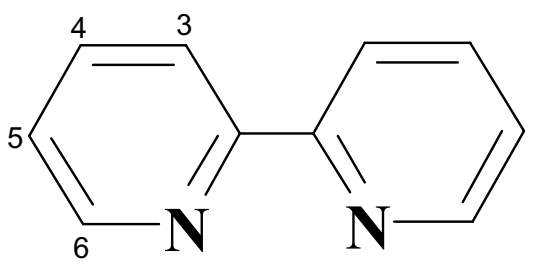

Antraquinona-2- $\mathrm{CO}_{2} \mathrm{H}$

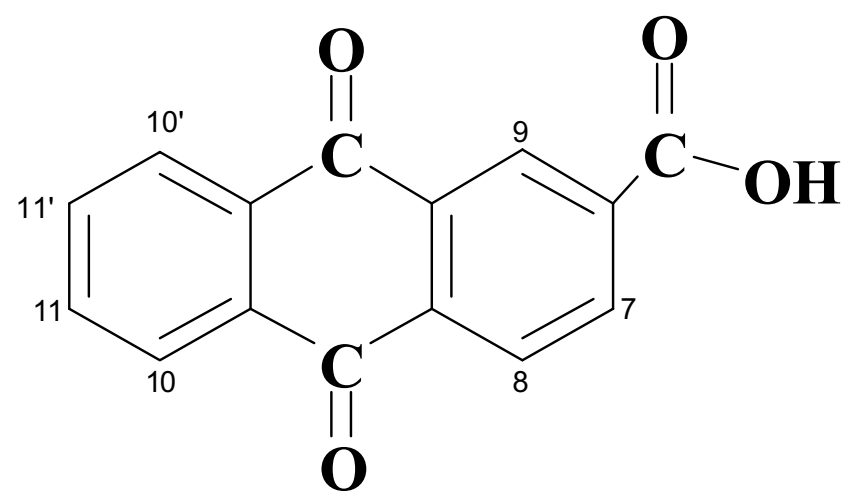


CAPITULO 2

\section{PREPARACION Y CARACTERIZACION DE} fac-[Antraquinona-2-COO-Re $e^{I}(\mathrm{CO})_{3}\left(2,2^{\prime}\right.$ 'bipiridina)] 


\section{Detalles Experimentales}

\section{Materiales}

Los solventes orgánicos y otros materiales utilizados cuya calidad no se especifica particularmente fueron de calidad proanálisis y se usaron sin purificación posterior. En los casos en que se requirió agua, ésta era bidestilada en un equipo Milli- $Q$. El nitrógeno utilizado fue de calidad ultrapura. Los compuestos ácido Antraquinona-2- $\mathrm{COOH}, 2,2$ ’bipiridina y hexafluorofosfato de tetrabutilamonio $\left(\left[\mathrm{N}\left(\mathrm{n}-\mathrm{C}_{4} \mathrm{H}_{9}\right)_{4}\right]\left(\mathrm{PF}_{6}\right), \mathrm{Bu}_{4} \mathrm{NPF}_{6}\right)$ fueron comprados a Aldrich. Esta última sustancia fue secada a $110{ }^{\circ} \mathrm{C}$ durante no menos de 24 hs. El acetonitrilo utilizado en las medidas electroquímicas, "SeccoSolv" de Merck, fue secado en óxido básico activo de aluminio 90 de la misma compañía durante varios días. El material de partida $\operatorname{BrRe}^{\mathrm{I}}(\mathrm{CO})_{5}$ fue comprado a Strem Chemicals y usado sin posterior purificación. El complejo precursor $f a c$-[BrRe $(\mathrm{CO})_{3}\left(2,2^{\prime}\right.$ 'bipi $\left.)\right]$ se obtuvo mediante la sustitución de 2 CO del $\operatorname{BrRe}^{\mathrm{I}}(\mathrm{CO})_{5}$ por la 2,2'bipi, siguiendo el protocolo que se describe en la bibliografía [10,11], aunque con pequeñas modificaciones que permitieron obtener mejores rendimientos.

\section{Síntesis y caracterización de $f a c$-[Antraquinona-2-COO-Re' $(\mathrm{CO})_{3}(2,2$ 'bipi)]}

El complejo de $\mathrm{Re}^{\mathrm{I}}$ en estudio, fue preparado modificando procedimientos que se encuentran en la literatura para complejos similares [10,11]. Así, la estrategia empleada para su obtención involucró la síntesis de los siguientes compuestos:

i. $\quad$ fac- $\left[\operatorname{BrRe}^{I}(\mathrm{CO})_{3}\left(2,2^{\prime}\right.\right.$ 'bipi $\left.)\right]$ 
Se mezclaron $3 \mathrm{~g}$ de $\operatorname{BrRe}^{\mathrm{I}}(\mathrm{CO})_{5}$ (7,4 mmoles) con 1,27 g de 2,2’bipi (8,1 mmoles) en $250 \mathrm{ml}$ de isooctano colocados en un balón, junto con un buzo magnético para su agitación. Se elimina el oxígeno deaereando con $\mathrm{N}_{2}$, y se calienta hasta ebullición en manto calefactor. La reacción procede durante 6 hs a reflujo, manteniendo la atmósfera de $\mathrm{N}_{2}$ y la agitación magnética constante. El producto precipita de la solución de isooctano y se lo aísla por filtración en vacío. El sólido obtenido se lava con $\mathrm{Et}_{2} \mathrm{O}$, se seca en estufa de vacío y se lo somete a recristalizaciones sucesivas hasta obtener valores del coeficiente de extinción $(\varepsilon)$ constante. La recristalización consiste en la disolución del sólido en la mínima cantidad de un solvente en el cual es soluble (DCM) y posterior precipitación por el agregado, gota a gota, de otro solvente, miscible con el primero pero en el cual el complejo es muy poco o nada soluble

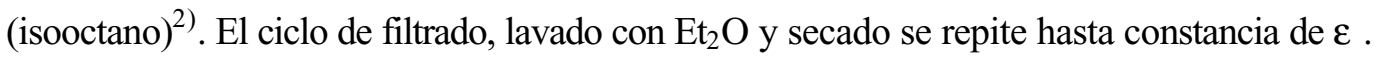

Se obtuvieron 3,67 g del complejo (sólido amarillo, Rto. 88 \%) y se lo utilizó como precursor sin otro paso de purificación.

\section{ii. Antraquinona-2-COOAg}

Con el fin de poder introducir al ligando aq-2- $\mathrm{CO}_{2}$ dentro de la esfera de coordinación del $\mathrm{Re}$, fue preparada la sal de $\mathrm{Ag}^{+}$del aq-2- $\mathrm{CO}_{2} \mathrm{H}$ por neutralización con $\mathrm{HKCO}_{3}$ y precipitación posterior, desde esta solución neutra, por adición de una cantidad estequiométrica de $\mathrm{NO}_{3} \mathrm{Ag}$ disuelta en una mínima cantidad de agua. Se deja agitando en la oscuridad durante 2 hs a 65 ${ }^{\circ} \mathrm{C}$, se enfría y el precipitado obtenido (fino polvo gris) se filtra y se seca en vacío (Rto. $\sim 100$ \%)

\footnotetext{
2) Todas las recristalizaciones realizadas en las síntesis se ajustaron a este procedimiento y aquellas en las que se ha recurrido a recristalizaciones diferentes serán convenientemente descriptas.
} 


\section{iii. fac-[Antraquinona-2-COO-Re ${ }^{I}(\mathrm{CO})_{3}\left(2,2^{\prime}\right.$ 'bipi $\left.)\right]$}

Los compuestos $\operatorname{BrRe}^{\mathrm{I}}(\mathrm{CO})_{3}\left(2,2^{2}\right.$ bipi $)$ y aq-2- $\mathrm{CO}_{2} \mathrm{Ag}$ se disolvieron en relación molar 1:1 en $100 \mathrm{ml}$ de DCM. Se mantuvo calentando a reflujo durante 10 horas, agitando y en atmósfera de $\mathrm{N}_{2}$. La solución y el sólido que contiene el complejo de $\operatorname{Re}^{\mathrm{I}}$ y el $\operatorname{BrAg}$ formado fueron colocados en un aparato de extracción Soxhlet y el producto soluble fue removido del sólido luego de 2 días de extracción. La solución fue concentrada por evaporación a presión reducida, se precipita el sólido por el agregado de isooctano y se seca en vacío. Luego se recristalizó varias veces en DCM / isooctano hasta constancia de los coeficientes de extinción. El sólido amarillo obtenido por filtración se lava con $\mathrm{Et}_{2} \mathrm{O}$ y se seca en vacío (Rto. > 70 \%) Análisis elemental. Calculado para $\mathrm{C}_{18} \mathrm{H}_{11} \mathrm{~N}_{4} \mathrm{O}_{5}$ Re: $\mathrm{C} \quad 49,55 \%, \mathrm{H} \quad 2,23 \%, \mathrm{~N} \quad 4,13 \%$. Encontrado: C 49,33 \%, H 2,22 \%, N 4,19\%

\section{Técnicas y equipamiento utilizados}

El análisis elemental fue realizado por Midwest Microlab, LLC (IN, USA).

Los espectros UV-visible fueron tomados en un espectrofotómetro de doble haz Cary 3 utilizando celdas de cuarzo de $1 \mathrm{~cm}$ de camino óptico y los espectros de infrarojo en un Shimadzu IR-435 usando pastillas obtenidas con mezclas del complejo de $\mathrm{Re}^{\mathrm{I}}$ en $\mathrm{BrK}$.

La resonancia magnética nuclear de hidrógeno, ${ }^{1} \mathrm{H}-\mathrm{RMN}$, se llevó a cabo en un equipo Bruker, Advance 400, equipado con gradientes de campo magnético, a $296 \mathrm{~K}$, utilizando soluciones preparadas con cloroformo deuterado, $\mathrm{Cl}_{3} \mathrm{CD}$.

Las medidas electroquímicas (voltametría cíclica de alterna (ac) y de continua (dc) fueron realizadas en un equipo Princeton Applied Research, PAR Model 173 con módulo de interface 276 A y Programador Universal Model 175. Los voltagramas ac fueron registrados 
con una amplitud de salida de $1,6 \mathrm{mV}_{\mathrm{rms}} / 6 \mathrm{~Hz}$ y una velocidad de barrido de $3,0 \mathrm{mV} \mathrm{cm} \mathrm{c}^{-1}$ (un ciclo). El $\mathrm{Bu}_{4} \mathrm{NPF}_{6}$ fue usado como electrolito soporte en concentración 0,1 $\mathrm{M}$ en ACN. Las medidas fue posible obtenerlas en un rango de potencial entre $-2,0$ y $+1,0 \mathrm{~V}$ vs SCE (electrodo de calomel saturado). Como electrodo de trabajo se usó un disco de Pt de $0,28 \mathrm{~cm}^{2}$ de área, cuya superficie fue pulida antes de cada experimento con alúmina (tamaño de partícula igual o menor a $0,05 \mu \mathrm{m}$ ) hasta obtención de un espejo. Posteriormente el electrodo fue enjuagado con agua Millipore Milli- $Q$, sonicado y secado antes de introducirlo en la solución. La calidad de la superficie y el funcionamiento del electrodo fue testeada ciclando brevemente en una solución de electrolito soporte. Como contraelectrodo se usó una malla de Pt, separada del compartimento principal por un disco poroso. Los potenciales se midieron frente al electrodo de referencia $\mathrm{Ag} / \mathrm{ClAg} / \mathrm{ClK}_{\text {sat }}$ y la exactitud de las medidas fue verificada usando la cupla $\mathrm{Fc} / \mathrm{Fc}^{+}$(ferroceno/ferrocinio) como referencia interna. Las soluciones fueron deareadas durante 30 minutos con corrientes de $\mathrm{N}_{2}$ seco antes de cada determinación. La concentración del complejo de $\operatorname{Re}^{\mathrm{I}}$ típicamente usada fue de $2 \times 10^{-4} \mathrm{M}$.

\section{Resultados}

\section{Síntesis}

La ruta de síntesis utilizada para la obtención del complejo de $\operatorname{Re}^{\mathrm{I}}$ se ilustra en el esquema I. La estrategia empleada para introducir el aq-2- $\mathrm{CO}_{2}$ en la esfera de coordinación del $\mathrm{Re}^{\mathrm{I}}$ involucró la utilización de la sal de $\mathrm{Ag}^{+}$del ácido carboxílico (etapa I). Esto era necesario ya que aumentaba la probabilidad de desplazar el $\mathrm{Br}^{-}$del complejo precursor al combinarse con el ion $\mathrm{Ag}^{+} \mathrm{y}$ formar el precipitado $\mathrm{BrAg}$. El primer paso para la obtención de la sal de $\mathrm{Ag}^{+}$ consistió en la neutralización del aq-2- $\mathrm{CO}_{2} \mathrm{H}$. Todos los antecedentes encontrados en la 
bibliografía (y trabajos anteriores en nuestro laboratorio) se ajustaba el ph a 7 con solución de OHNa [10,11], pero en este caso el OHNa reaccionaba con la quinona. Para evitar tales efectos secundarios se neutralizó utilizando $\mathrm{KHCO}_{3}$. La precipitación posterior con $\mathrm{NO}_{3} \mathrm{Ag}$ se debe realizar en la oscuridad para evitar la reducción de $\mathrm{Ag}^{+}$. Se obtiene un precipitado en forma de polvo de color gris en cantidades estequeométricas. En la etapa II se describe la síntesis del complejo precursor de $\mathrm{Re}^{\mathrm{I}}$ ampliamente utilizado y descripto en la literatura [1214]. Los espectros UV-visible conocidos para este complejo, permitieron verificar que fue obtenido con éxito, con un alto rendimiento y con la pureza adecuada para utilizarlo en el próximo paso de síntesis (etapa III). Esta etapa es la síntesis propiamente dicha del complejo de $\operatorname{Re}^{\mathrm{I}}$ deseado. Fueron necesarios varios intentos con diversas condiciones experimentales ( $\mathrm{T}$, tiempo de reacción, burbujeo constante con $\mathrm{N}_{2}$, etc.) así como el empleo de diferentes solventes como tolueno, ACN e isooctano. La obtención del producto demandó, en consecuencia, tiempo y esfuerzo. Cuando el ligando a introducir fue el 4-Nitrobenzoato el solvente adecuado fue $\mathrm{ACN}$, sin embargo para el ligando piracinacarboxilato la obtención se hizo en tolueno [10,11]. Otros carboxilatos sintetizados recientemente por nuestro grupo fue posible obtenerlos en DCM [15]. Este fue el solvente adecuado también para el complejo con aq-2- $\mathrm{CO}_{2}$. Las características estructurales de este ligando es responsable de la baja solubilidad encontrada en los diferentes solventes empleados y que dificultan el trabajo experimental. Esta baja solubilidad se observa también en el complejo comparada con otros complejos similares ${ }^{3)}$. Aún en DCM, la separación del BrAg precipitado, solo pudo lograrse luego de 2 días de extracción continua en Soxhlet debido a la coprecipitación del complejo con el BrAg.

\footnotetext{
3) Tal vez esta sea la causa principal que imposibilita la obtención de monocristales del complejo como se había obtenido en otros casos, ya que el método usual para generarlos es, partir de una solución concentrada y posteriormente permitir la evaporación lenta del solvente utilizado. Para este fin se usan finos tubos con tapón de goma tipo septum sobre el cual se atraviesa una aguja que conecte al exterior y permita la salida lenta de las moléculas del solvente
} 
Finalmente, el complejo se obtuvo con alto rendimiento, mostrando una muy buena concordancia entre los valores calculados y encontrados en los porcentajes de $\mathrm{C}, \mathrm{H}$ y $\mathrm{N}$ del análisis elemental. Son los resultados de análisis elemental una fuerte evidencia del éxito de la síntesis. 
Esquema I: Ruta de síntesis para la obtención del aq-2-CO $\mathrm{CO}_{2}-\operatorname{Re}^{\prime}(\mathrm{CO})_{3}\left(2,2^{\prime}\right.$ bipi)

I<smiles>[X]C(=O)O</smiles><smiles>[X]=[X]</smiles>

II

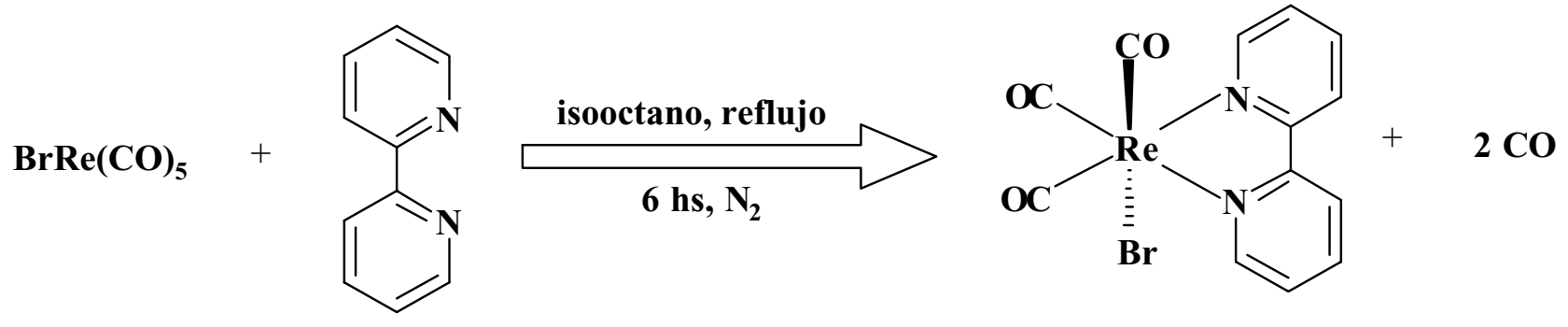

III<smiles>[Y]C(=O)O[W]</smiles> 


\section{Espectros de absorción infraroja}

La estructura cristalina de varios complejos relacionados han mostrado que el entorno del átomo de $\mathrm{Re}^{\mathrm{I}}$ central es del tipo octahédrico distorsionado. En particular para los $\mathrm{L}-\mathrm{CO}_{2}$ $\operatorname{Re}^{\mathrm{I}}(\mathrm{CO})_{3}(2,2$ 'bipi) ( $\mathrm{L}=$ piracina, antraceno) se ha establecido que el Re está coordinado por dos grupos carbonilos y por los nitrógenos de la 2,2'bipi (ligando bidentado) definiendo un plano ecuatorial $[10,11]$. La coordinación octahédrica se completa en la posición axial por otro carbonilo y por el oxígeno del $\mathrm{L}_{-} \mathrm{CO}_{2}-$. Los tres $\mathrm{CO}$ se encuentran sobre los vértices de una de las caras del octahedro, en una conformación que se denomina de tipo facial ${ }^{4}$. Nosotros asumiremos el mismo entorno octahédrico distorsionado y de hecho, el patrón de bandas del espectro IR, figura 1, en la zona comprendida entre 1800 y $2100 \mathrm{~cm}^{-1}$, se corresponde con las tres bandas características asignadas al estiramiento de los CO en posición facial. Para el complejo de $\mathrm{Re}^{\mathrm{I}}$ en estudio, se observan bandas a 1896, 1916 y 2025 $\mathrm{cm}^{-1}$. En general, para complejos del tipo $\mathrm{L}-\mathrm{Re}^{\mathrm{I}}(\mathrm{CO})_{3}(\mathrm{NN})$, donde $\mathrm{NN}$ es un bidentado polipiridínico, este patrón de bandas se ve levemente modificado en función de la naturaleza del ligando L. Así, cuando $\mathrm{L}=\mathrm{Cl}, \mathrm{Br}, \mathrm{PPh}_{3}$ (trifenilfosfina), etc, las tres bandas están bien definidas y presentan alta intensidad [12]. Sin embargo, cuando $\mathrm{L}=\mathrm{CH}_{3} \mathrm{CN}$, piridina, 4etilpiridina, N-metilimidazol, etc, la banda de menor energía se hace menos notable hasta desaparecer por completo [16]. Esto se debe a la menor o mayor simetría que adquiere el octahedro para los diferentes ligandos L y que impacta en forma directa en los diferentes modos vibracionales de los carbonilos en la esfera de coordinación del Re. Así, cuando L es un derivado piridínico la estructura obtiene una alta simetría local sobre el átomo central ya que el complejo posee tres $\mathrm{CO}$ y tres ligandos "piridílicos equivalentes" en arreglo facial y lo

\footnotetext{
4) De ahí surge el prefijo fac- que acompaña a todos los complejos de $\mathrm{Re}^{\mathrm{I}}$ tricarbonílicos que presenten, o a los cuales se considere, esta conformación
} 
que se observa es un patrón de solo 2 bandas. Esto se ha observado también en complejos del tipo $\operatorname{Re}^{\mathrm{I}}(\mathrm{CO})_{3} \mathrm{~L}_{3}{ }^{+}$donde $\mathrm{L}=$ piridina sustituida [13]. Por el contrario, cuando $\mathrm{L}=\mathrm{Cl}$ se han observado las tres bandas correspondientes en 2011, 1924, y $1902 \mathrm{~cm}^{-1}$ [16]. Con nuestro complejo nos encontramos en una situación intermedia ya que la banda de $1916 \mathrm{~cm}^{-1}$ se puede apreciar pero en forma de un débil hombro sin definirse como pico pero tampoco mostrando una banda única. De manera que, en principio, podríamos asumir una estructura menos distorsionada en nuestro complejo comparada con el complejo precursor y con una conformación acorde con la ilustración mostrada en el esquema I.

Figura 1: Espectro IR del aq-2- $\mathrm{CO}_{2}-\operatorname{Re}^{\prime}(\mathrm{CO})_{3}\left(2,2^{\prime}\right.$ 'bipi)

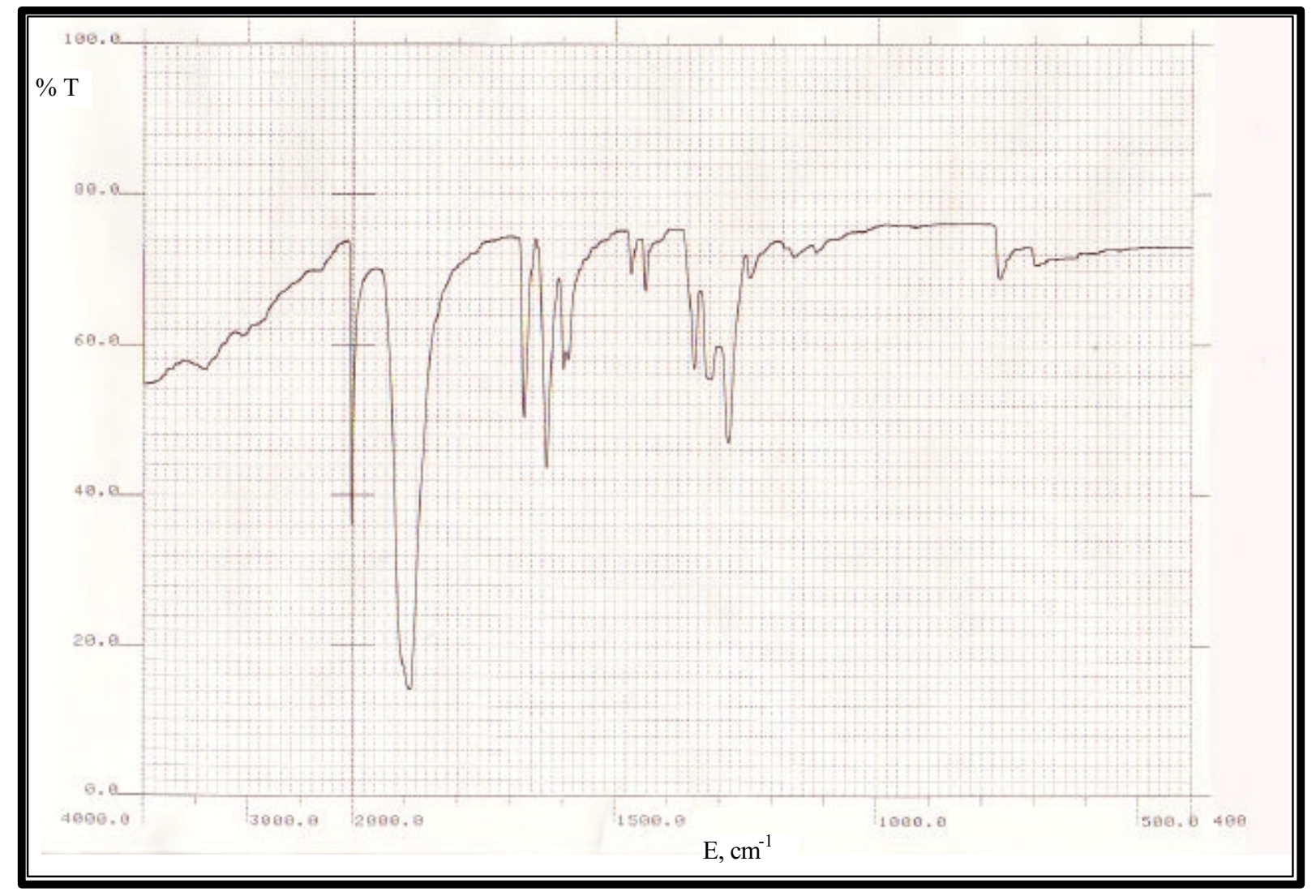




\section{Espectros ${ }^{1}$ H-RMN}

El espectro de ${ }^{1} \mathrm{H}-\mathrm{RMN}$ del aq-2- $\mathrm{CO}_{2}-\mathrm{Re}^{\mathrm{I}}(\mathrm{CO})_{3}\left(2,2^{\prime}\right.$ 'bipi) fue obtenido realizando varios barridos sucesivos debido a la baja solubilidad del complejo en el $\mathrm{Cl}_{3} \mathrm{CD}$ utilizado como solvente. Como consecuencia de ello en el espectro se acentúan dos pequeñas señales correspondientes a impurezas del solvente que se han registrado separadamente en forma previa y que no presentan correlación en un espectro de tipo bidimensional, COSY. En la figura 2 se muestra el espectro correspondiente donde se observa que las señales de los ligandos 2,2'bipi y aq-2- $\mathrm{CO}_{2}$ - aparecen superpuestas en una zona muy angosta del espectro protónico y no pueden ser diferenciadas fácilmente. La integración específica para varios protones no puede ser determinada con exactitud. Sin embargo, si se considera que la señal más desprotegida corresponde a 2 protones, la suma global de las integraciones corresponde a 15 protones, tal como se espera (ver la numeración asignada para cada protón en los correpondientes ligandos, Introducción Parte II). La asignación de estos protones se realizó asumiendo la conformación considerada en la discusión de la sección anterior.

En principio, puede decirse que los tres protones 7,8 y 9 poseen desplazamientos parecidos. Los H7 y H8 forman un sistema que debería observarse como dos dobletes. El doblete del protón $\mathrm{H} 7$ debería, a su vez, estar desdoblado en un doble doblete (dd), por acoplamiento a cuatro enlaces con el protón H9. Por otro lado, éste H9, debería aparecer desdoblado con una constante de acoplamiento pequeña por efecto del H7.

Para los protones 10 y 11 se espera un sistema similar a H7 y H8 (doblete para cada señal). En el espectro se observan que los picos de estos dobletes están desdoblados (dd, cuatro señales) indicando que los protones 10 y 11 no son exactamente equivalentes generando un sistema en el que los protones han sido denominados ahora como H10, H10', H11 y H11'. Es decir que el protón 10 acopla separadamente con el 11 y 11' (y lo mismo para la señal del 11). 
El acoplamiento de los cuatro protones de la 2,2'bipi sigue un patrón observado en otros complejos similares del tipo $-\operatorname{Re}^{\mathrm{I}}(\mathrm{CO})_{3}\left(2,2^{\prime}\right.$ bipi) [15] y en la asignación de cada protón se tuvieron en cuenta los desdoblamientos de las señales generadas como consecuencia de la mútua interacción entre ellos. Así, las asignaciones de los protones dentro de cada sistema fueron realizadas en consideración al entorno químico particular de cada protón.

Los protones $\mathbf{H 6}$ corresponderían al doblete doblete que aparece a menor campo (en 9,262 ppm) pues se presume que sería afectado estéricamente por el entorno del metal y por las corrientes paramagnéticas desprotectoras del triple enlace $\mathrm{C} \equiv \mathrm{O}$ dispuestos en el mismo plano de la 2,2’bipi, y que estaría en concordancia con la conformación espacial asumida en el análisis de los datos de IR (ver sección anterior). Siguiendo con esta consideración, los protones $\mathbf{H 3}$ corresponden al otro dd del sistema ubicado a mayor campo (en 8,276 ppm)

De ambos seudotripletes desdoblados, el que aparece a menor campo correspondería a los protones $\mathbf{H 4}$ (en 8,150 ppm). El efecto para del nitrógeno heteronuclerar que se encuentra enlazado al metal causaría que este protón esté más desprotegido que el $\mathbf{H 5}$ (en 7,594 ppm). Esto también estaría de acuerdo con el pico de acoplamiento mas intenso entre la señal del H6 y el H5 que se observa en el COSY.

Los protones correspondientes a la aq-2- $\mathrm{CO}_{2}$ fueron asignados en base al análisis explicado previamente. Así, el protón $\mathbf{H 9}$ correspondería a la señal más aguda que aparece superpuesta con parte de la señal correspondiente al H4. El sistema compuesto por los protones $\mathbf{H 7}$ y $\mathbf{H 8}$ presentan desplazamientos en 8,069 ppm y 8,111 ppm. La señal en 8,069 ppm aparece además desdoblada y es, por lo tanto, asignada al protón $\mathbf{H 7}$ mientras que la señal en 8,111 ppm correspondería al protón $\mathbf{H 8}$.

Por último, los protones $\mathbf{H 1 0}$ y $\mathbf{H 1 0}$ ' aparecen a campos más altos (en 7,756 ppm) que los protones H11 y H11' (en 8,236 ppm). Esto es debido a la protección ejercida por las 
corrientes paramagnéticas del doble enlace $\mathrm{C}=\mathrm{O}$, ya que están ubicados en la molécula de tal modo que quedan en la zona de protección de estas corrientes electrónicas.

Las señales obtenidas y analizadas en esta sección permiten inferir que los ligandos aq-2- $\mathrm{CO}_{2}$ y 2,2'bipi forman parte del complejo sintetizado y que no existen señales atribuibles a la presencia de impurezas.

Figura 2: Espectro ${ }^{1} \mathrm{H}-\mathrm{RMN}$ del aq-2- $\mathrm{CO}_{2}-\mathrm{Re}^{\prime}(\mathrm{CO})_{3}\left(2,2{ }^{\prime}\right.$ bipi) en $\mathrm{Cl}_{3} \mathrm{CD}$

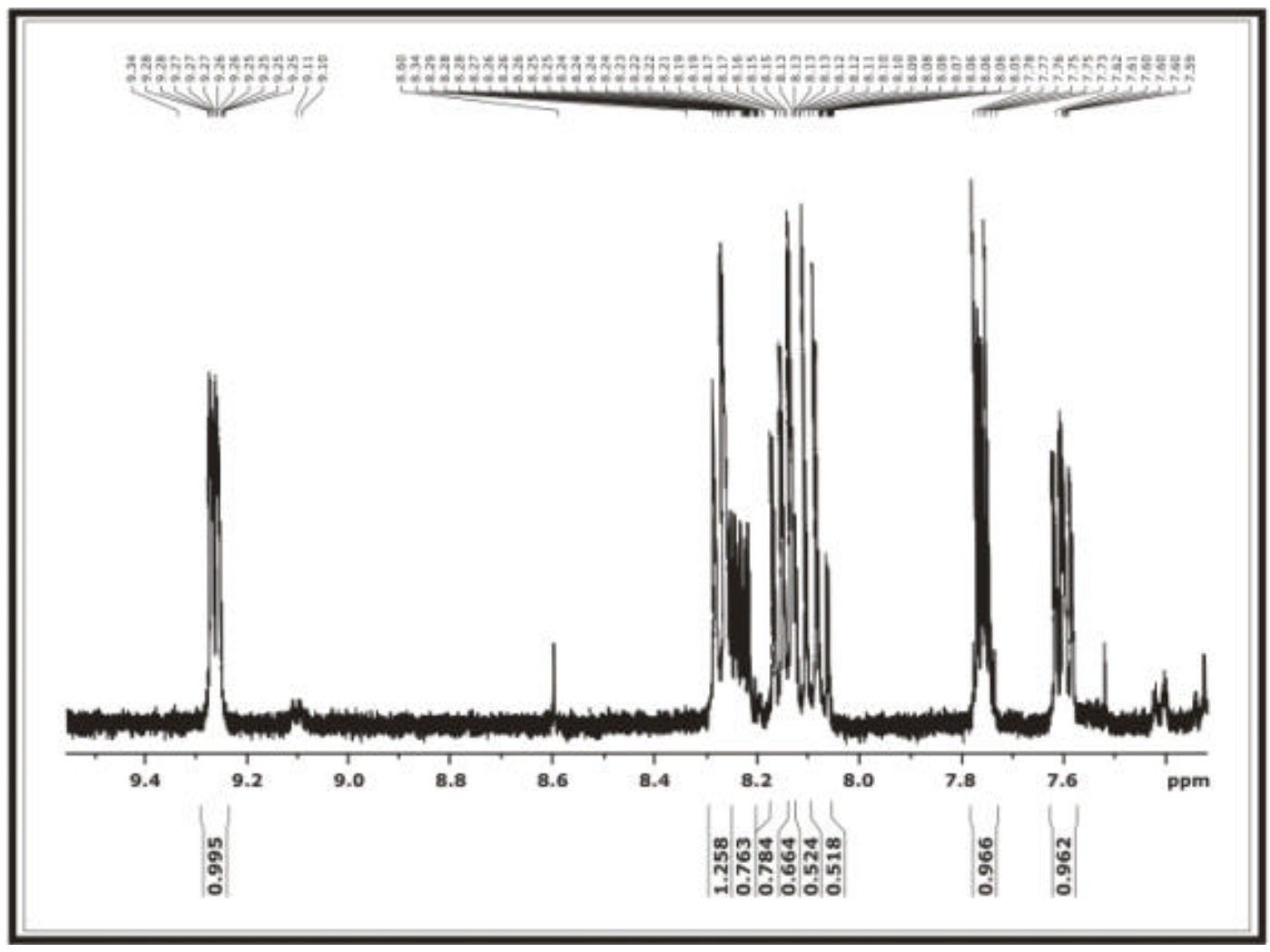




\section{Espectros de absorción UV-visible}

En la figura 3 se muestran los espectros UV-visible en $\mathrm{CH}_{3} \mathrm{CN}$ y $\mathrm{CH}_{2} \mathrm{Cl}_{2}$ del aq-2- $\mathrm{CO}_{2}$ $\operatorname{Re}^{\mathrm{I}}(\mathrm{CO})_{3}\left(2,2^{\prime}\right.$ bipi), siendo similares a los espectros de complejos que contienen al cromóforo $\left[\operatorname{Re}^{\mathrm{I}}(\mathrm{CO})_{3}\left(2,2^{\prime} \text { bipi }\right)\right]^{+} \quad[10,11,17] . \quad$ Se muestra también el correspondiente al $\operatorname{BrRe}^{\mathrm{I}}(\mathrm{CO})_{3}\left(2,2^{\prime}\right.$ bipi) y al aq- $\mathrm{CO}_{2} \mathrm{H}$ en $\mathrm{ACN}$.

La primer banda de absorción, $\lambda_{\text {máx }} \approx 400 \mathrm{~nm}$, puede ser asignada a una transición que corresponde a una transferencia de carga $\mathrm{d} \pi\left(\operatorname{Re}^{\mathrm{I}}\right) \rightarrow \pi^{*}\left(2,2^{\prime}\right.$ bipi); ésta se observa en el complejo precursor y presenta un corrimiento hacia energías menores cuando disminuye la polaridad del solvente. Esto es característico de transiciones que provocan un cambio en el momento dipolar de la molécula. En este caso la transferencia de carga ocurrida durante la transición conduce a un estado excitado cuyo momento dipolar es menor que el del estado fundamental [18]. 


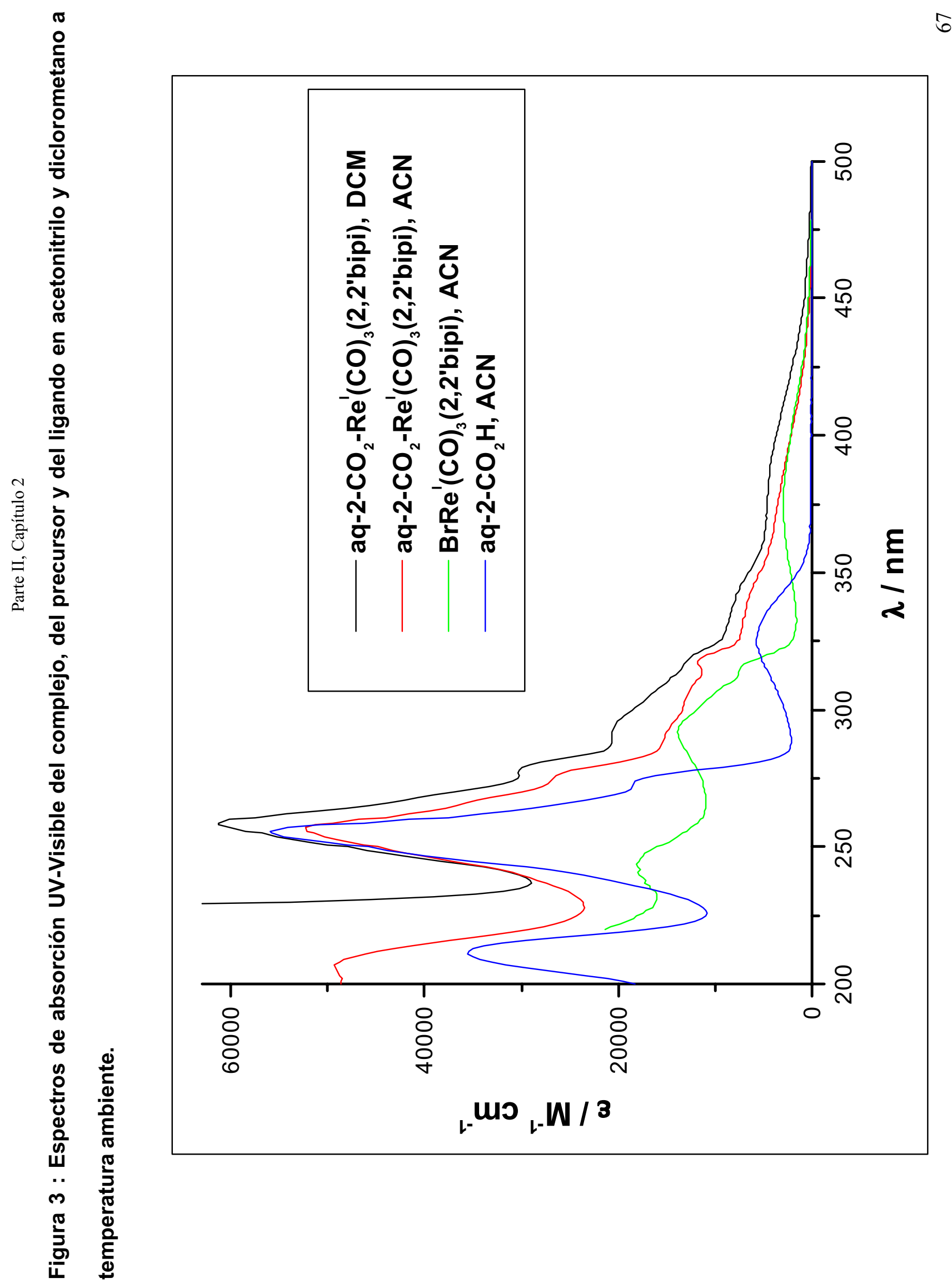


Sin embargo, en este tipo de complejos no se descarta la posibilidad de solapamiento de esta banda con otras de tipo intraligando centradas en la 2,2'bipi.

En la región UV cercano $\left(\lambda \approx 260 \mathrm{~nm}\right.$ ) las transiciones electrónicas del tipo $\pi \rightarrow \pi^{*}$ centradas en los ligandos dominan el espectro de absorción. El $\operatorname{BrRe}^{\mathrm{I}}(\mathrm{CO})_{3}(2,2$ ’bipi) presenta un máximo de absorción a $\lambda=292 \mathrm{~nm}$ con un $\varepsilon \approx 1,4 \cdot 10^{4} \mathrm{M}^{-1} \mathrm{~cm}^{-1}$ que correspondería a una transición $\pi \rightarrow \pi^{*}$ centrada fundamentalmente en la 2,2'bipi [19]. Los rasgos de esta transición se distinguen perfectamente, en el complejo de $\mathrm{Re}^{\mathrm{I}}$ en estudio, en forma de hombro. La aq-2- $\mathrm{CO}_{2} \mathrm{H}$, por otra parte, muestra un máximo de absorción a $\lambda=255 \mathrm{~nm}, \varepsilon \approx 5,6.10^{4} \mathrm{M}^{-1}$ $\mathrm{cm}^{-1}$, correspondiendo a una transición $\pi \rightarrow \pi^{*}$ centrada en este ligando. En el complejo con aq-2- $\mathrm{CO}_{2-}$, el máximo se encuentra a $\lambda=257 \mathrm{~nm}$ con un $\varepsilon \approx 5,8.10^{4} \mathrm{M}^{-1} \mathrm{~cm}^{-1}$, reflejando la superposición con otras transiciones intraligando, y predominando, en este complejo, la correspondiente a la aq-2- $\mathrm{CO}_{2}$. La banda observada a $211 \mathrm{~nm}$ también puede asignarse a una transición intraligando. Estas transiciones no muestran un gran efecto de la polaridad del solvente, aunque el alto valor de los coeficientes de absorción molar permite identificar estas transiciones como de tipo $\pi \rightarrow \pi^{*}$ [18]. Otras bandas presentes en la zona entre 280 y $350 \mathrm{~nm}$ fueron asignadas a transiciones solapadas centradas en la aq-2- $\mathrm{CO}_{2}$ y en la 2,2'bipi por comparación con las observadas en sus espectros. En la tabla I se resumen las bandas de absorción del complejo y de los ligandos. 
Tabla I: Bandas de absorción presentes en los complejo de $\operatorname{Re}^{\prime}$ y en el ligando

\begin{tabular}{|c|c|c|}
\hline Compuesto & $\lambda^{\mathrm{a}} / \mathrm{nm} ; \varepsilon / \mathrm{M}^{-1} \mathrm{~cm}^{-1}$ & Asignación \\
\hline \multirow[t]{4}{*}{$\mathrm{aq}-2-\mathrm{CO}_{2}-\mathrm{Re}^{\mathrm{I}}(\mathrm{CO})_{3}\left(2,2^{\prime} \mathrm{bipi}\right)$} & $\begin{array}{l}208,48777 \\
257,52114 \\
276,26365 \\
292,15200 \\
318,8930 \\
336,6760\end{array}$ & $\pi \rightarrow \pi^{*}$ \\
\hline & $\approx 372,3597$ & $\mathrm{~d} \pi \rightarrow \pi^{*}$ \\
\hline & $\begin{array}{l}259^{\mathrm{b}}, 61243 \\
278,29710 \\
292,20787 \\
319,12800 \\
337,8229\end{array}$ & $\pi \rightarrow \pi^{*}$ \\
\hline & $\approx 388,4311$ & $\mathrm{~d} \pi \rightarrow \pi^{*}$ \\
\hline \multirow[t]{3}{*}{$\operatorname{BrRe}^{I}(C O)_{3}\left(2,2^{\prime}\right.$ bipi $)$} & & \\
\hline & $\begin{array}{l}243,17992 \\
292,13890 \\
315,7478\end{array}$ & $\pi \rightarrow \pi^{*}$ \\
\hline & 377,2956 & $\mathrm{~d} \pi \rightarrow \pi^{*}$ \\
\hline \multirow[t]{2}{*}{$\mathrm{aq}-2-\mathrm{CO}_{2} \mathrm{H}$} & & \\
\hline & $\begin{array}{l}212,35473 \\
256,55864 \\
273,18471 \\
327,5694\end{array}$ & $\pi \rightarrow \pi^{*}$ \\
\hline
\end{tabular}

a. Todos los valores corresponden a espectros a temperatura ambiente en acetonitrilo.

b. En diclorometano 


\section{Medidas electroquímicas}

En la figura 4a se muestra la voltametría cíclica de continua (dc) de una solución deaereada $2,1.10^{-4} \mathrm{M}$ de aq-2- $\mathrm{CO}_{2}-\mathrm{Re}^{\mathrm{I}}(\mathrm{CO})_{3}\left(2,2^{\prime}\right.$ bipi) en $\mathrm{ACN}$ utilizando como electrolito soporte $\mathrm{Bu}_{4} \mathrm{NPF}_{6}$ 0,1 M. El barrido catódico ( $0 /-2 \mathrm{~V}$ ) a distintas velocidades permite distinguir al menos dos ondas de reducción correspondientes a la adición sucesiva de dos electrones. El primer pico de reducción se puede caracterizar con un valor de potencial de media onda $\left(\mathrm{E}_{1 / 2}\right)$ de $-850 \mathrm{mV}$ con respecto al electrodo de $\mathrm{Ag} / \mathrm{ClAg} / \mathrm{ClK}_{\text {sat }}(-60 \mathrm{mV}$ vs $\mathrm{SCE})$ y corresponde a un proceso reversible ${ }^{5)}$. A potenciales más negativos se observa una onda que permite vislumbrar los rasgos de otra solapada con ésta y que podría corresponder a dos procesos de reducción que ocurren a potenciales cercanos $y$ donde no se puede definir un $\mathrm{E}_{1 / 2}$ determinado. Para hacerlo se recurrió a la voltametría cíclica de alterna (ac) la que permite establecer con mayor precisión los E [20]. En la figura $4 \mathrm{~b}$ se muestra la voltametría cíclica ac de una solución $2.10^{-4} \mathrm{M}$ de aq-2-CO $-\mathrm{CO}_{2}-\mathrm{Re}^{\mathrm{I}}(\mathrm{CO})_{3}\left(2,2^{\prime}\right.$ bipi) en $\mathrm{ACN}$. La respuesta de la corriente muestra claramente tres picos a $-0,79,-1,21$ y $-1,33 \mathrm{~V}$ con respecto al electrodo de $\mathrm{Ag} / \mathrm{ClAg} / \mathrm{ClK}_{\text {sat }}$ correspondiente a la adición sucesiva y reversible de tres electrones. Un pico adicional fue observado en barrido anódico a $+1,42 \mathrm{~V}$ y fue asignado a la cupla $\mathrm{Re}^{\mathrm{II}} / \mathrm{Re}^{\mathrm{I}}$ por comparación con los potenciales observados para la misma cupla en varios complejos de $\mathrm{Re}^{\mathrm{I}}$ relacionados [11,21]. Bajo condiciones similares, figura 5, el voltagrama ac para una solución $2.10^{-4} \mathrm{M}$ de $\operatorname{BrRe}^{\mathrm{I}}(\mathrm{CO})_{3}(2,2$ 'bipi), mostró dos picos de reducción a $-1,26$ y $-1,46 \mathrm{~V}$ que fueron asignados a la reducción del ligando coordinado 2,2'bipi / 2,2'bipi-', como en complejos similares [11]. Por otro lado, en el voltagrama ac correspondiente a una solución $1.10^{-4} \mathrm{M}$ del

\footnotetext{
5) Se define $\mathrm{E}_{/ / 2}$ como el promedio entre el $\mathrm{E}_{\text {anódico }} \mathrm{y}$ el $\mathrm{E}_{\text {catódico }}$ de la onda en voltametría cíclica dc. El criterio asumido para considerar proceso reversible fue que $\mathrm{E}_{\text {catódico }}-\mathrm{E}_{\text {anódico }} \quad 60 / \mathrm{n} \mathrm{mV}$, donde $\mathrm{n}=$ nro. de electrones involucrados en el proceso. En el caso de voltametría cíclica ac se considera onda reversible cuando la curva del barrido de ida coincide con la del barrido de vuelta y, entonces, el $\mathrm{E}_{\mathrm{pico}}$ (máximo de la curva) $=\mathrm{E}_{1 / 2}$. Además el ancho del pico (definido como la distancia en la altura media), debe ser ùp $\quad 90 / \mathrm{n} \mathrm{mV} \mathrm{[20]}$
} 
ligando libre aq-2- $\mathrm{CO}_{2} \mathrm{H}$, figura 5 , se observaron picos a $-0,93$ y $-1,39 \mathrm{~V}$ que corresponden a la adición sucesiva de dos electrones. Las ondas reversibles observadas en voltametría cíclica dc se encontraron a los mismos potenciales que en la voltametría ac.

Figura 4: Voltametría cíclica del aq-2-CO $-\mathrm{Ce}^{\prime}(\mathrm{CO})_{3}\left(2,2^{\prime} \mathrm{bipi}\right)$ en $\mathrm{ACN}$. a) dc y b) ac.
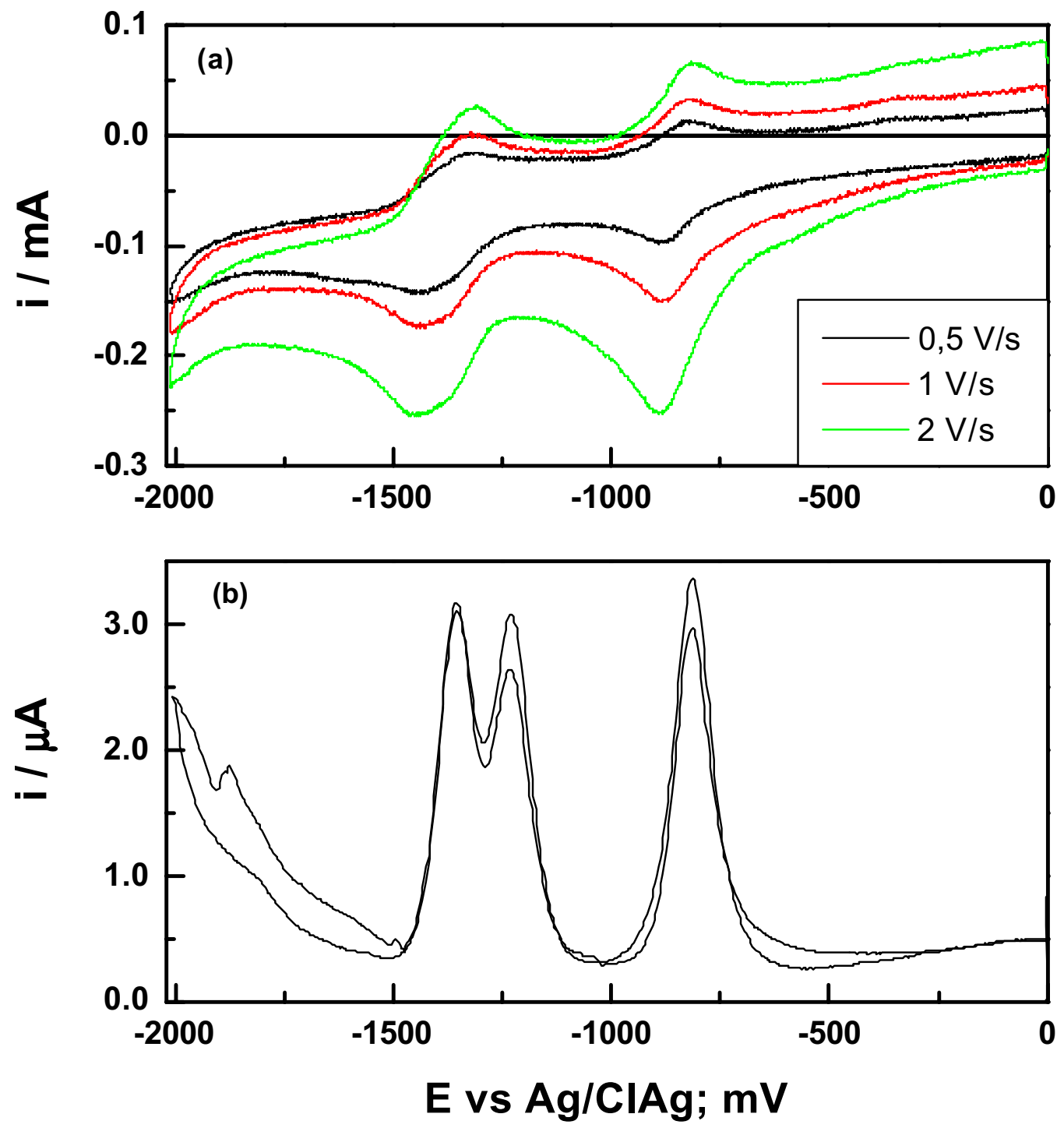
Figura 5: Voltametría cíclica ac de los ligandos en ACN.
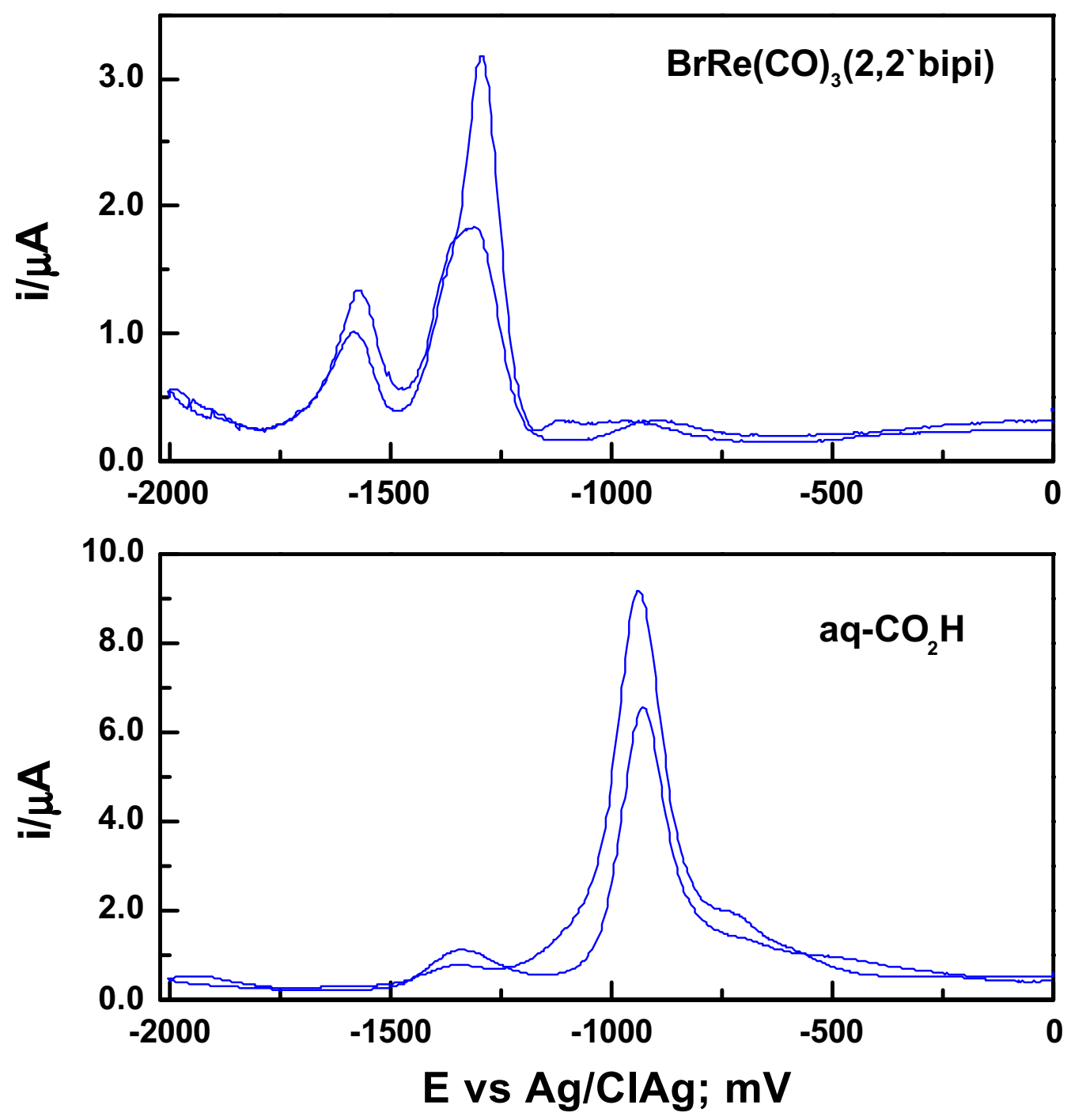

La comparación de los voltagramas de la figura 4 con la figura 5 indica que las reducciones a $-0,79$ y $-1,21 \mathrm{~V}$ ocurren sobre el ligando de antraquinona mientras que el proceso a $-1,33 \mathrm{~V}$ lo hace sobre la 2,2'bipi. Los potenciales de reducción para el aq-2- $\mathrm{CO}_{2} \mathrm{H}$ se encontraron que eran más negativos comparados con los potenciales correspondientes cuando el ligando está complejado en el aq-2-CO $\mathrm{CO}_{2}-\mathrm{Re}^{\mathrm{I}}(\mathrm{CO})_{3}\left(2,2^{\prime}\right.$ bipi). Esto puede racionalizarse considerando que el ligando aq-2- $\mathrm{CO}_{2}$ - en el complejo comparte la densidad electrónica con el centro de $\mathrm{Re}^{\mathrm{I}}$ 
debido a la coordinación convirtiéndose en un mejor receptor de electrones. La misma comparación puede hacerse para la reducción del ligando 2,2’bipi. En este caso la situación es inversa, es decir, el potencial de reducción del ligando coordinado en el aq-2- $\mathrm{CO}_{2}$ $\operatorname{Re}^{\mathrm{I}}(\mathrm{CO})_{3}\left(2,2^{\prime}\right.$ bipi) fue encontrado a valores más negativos que en el complejo precursor, $\operatorname{BrRe}^{\mathrm{I}}(\mathrm{CO})_{3}\left(2,2^{\prime}\right.$ bipi). Esto podría estar relacionado con la más alta densidad de carga positiva sobre el centro de $\mathrm{Re}^{\mathrm{I}}$ en el aq-2- $\mathrm{CO}_{2}-\mathrm{Re}^{\mathrm{I}}(\mathrm{CO})_{3}\left(2,2^{\prime}\right.$ bipi) que en el complejo $\operatorname{BrRe}^{\mathrm{I}}(\mathrm{CO})_{3}\left(2,2^{\prime}\right.$ 'bipi) debido a la presencia del grupo atractor de electrones carboxilato.

Los resultados de las medidas electroquímicas se muestran en la tabla II

Tabla II: Potenciales electroquímicos de los complejo de $\operatorname{Re}^{\prime}$ y del ligando

\begin{tabular}{|c|c|c|c|c|}
\hline & \multicolumn{4}{|c|}{${ }^{\mathrm{a}} \mathrm{E}_{1 / 2} / \mathrm{V}$ vs $\mathrm{Ag} / \mathrm{ClAg}$} \\
\hline & $+3 e$ & $+2 \mathrm{e}$ & $+1 \mathrm{e}$ & $-1 \mathrm{e}$ \\
\hline $\operatorname{BrRe}^{\mathrm{I}}(\mathrm{CO})_{3}\left(2,2^{\prime}\right.$ 'bipi $)$ & & $-1,46$ & $-1,26$ & \\
\hline $\mathrm{aq}-2-\mathrm{CO}_{2}-\operatorname{Re}^{\mathrm{I}}(\mathrm{CO})_{3}\left(2,2^{\prime}{ }^{\prime} \mathrm{bipi}\right)$ & $-1,33$ & $-1,21$ & $-0,79$ & $+1,42$ \\
\hline $\mathrm{aq}-2-\mathrm{CO}_{2} \mathrm{H}$ & & $-1,39$ & $-0,93$ & \\
\hline
\end{tabular}

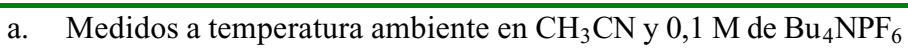




\section{Conclusiones}

El complejo de $\operatorname{Re}^{\mathrm{I}}$ aq-2- $\mathrm{CO}_{2}-\mathrm{Re}^{\mathrm{I}}(\mathrm{CO})_{3}\left(2,2^{\prime}\right.$ bipi), ha sido sintetizado con alto rendimiento y pureza. Los buenos resultados del análisis elemental evidencian la pureza del compuesto. Pero el resto de las determinaciones espectroscópicas confirman esta presunción y aportan información de valor sobre la disposición espacial de los ligandos en la esfera de coordinación del $\mathrm{Re}^{\mathrm{I}}$. El espectro ${ }^{1} \mathrm{H}-\mathrm{RMN}$ presenta desplazamientos de los protones acordes con la asignación prevista en función de la conformación de los ligandos aromáticos propuesta. El espectro infrarrojo nos proporciona evidencia concreta de la disposición de los $\mathrm{CO}$ en el complejo. El patrón de bandas en la zona de estiramiento de éstos es típica de tres $\mathrm{CO}$ en ubicación facial, compartiendo los vértices de una cara del octahedro, rasgo que presentan muchos complejos de $\operatorname{Re}^{\mathrm{I}}$ relacionados. Las bandas de absorción en el UV-visible muestran la presencia de absorciones que existen en los ligandos por separado y que en el complejo se observan superpuestas. En algunos casos, los valores de $\varepsilon$ son la suma de los $\varepsilon$ de los ligandos separados. Esta observación también aporta evidencia de la presencia de los ligandos en la esfera de coordinación del $\mathrm{Re}^{\mathrm{I}}$.

Por último, las medidas electroquímicas permitieron caracterizar el complejo con sus potenciales de media onda, que corroboran la presencia de los ligandos en el complejo, con ondas de reducción que fácilmente se pueden asignar a procesos reductivos que ocurren sobre cada uno de los ligandos y fundamentalmente, la ausencia de otras que descartan la presencia de impurezas.

El conjunto de estos resultados nos revela que el complejo fue obtenido con éxito y con un alto grado de pureza y fue utilizado sin otro tratamiento en los estudios fotoquímicos y fotofísicos posteriores. 
CAPITULO 3

\section{PROPIEDADES FOTOQUÍMICAS Y FOTOFÍSICAS DE fac-[Antraquinona-2-COO-Re $e^{I}(\mathrm{CO})_{3}\left(2,2^{\prime}\right.$ bipiridina $\left.)\right]$}




\section{Resultados experimentales y Discusión}

\section{Materiales}

Los solventes orgánicos, el complejo de $\mathrm{Re}^{\mathrm{I}}$, su precursor y ligandos que fueron utilizados, se obtuvieron como se describe en el capítulo 2. Las aminas alifáticas trietanolamina y trietilamina fueron compradas a Aldrich y se usaron sin posterior purificación.

\section{Equipos y métodos}

La luminiscencia de los complejos de $\mathrm{Re}^{\mathrm{I}}$ en estado estacionario fueron obtenidos en un espectrofluorómetro Perkin-Elmer LS 50B. Los espectros fueron corregidos por la diferente respuesta del instrumento y por la luz dispersada. Las soluciones fueron deareadas con $\mathrm{N}_{2}$ libre de $\mathrm{O}_{2}$ mediante el uso de tubos de teflon introducidos, a través del robinete, en celdas de cuarzo rectangulares de $1 \mathrm{~cm}$ x $1 \mathrm{~cm}$, antes de cada corrida. Los rendimientos cuánticos de emisión fueron medidos relativos a la Rodamina B en etanol absoluto $\left(\phi_{\mathrm{emi}}=0,69\right.$ [22]) y calculados de acuerdo con la siguiente ecuación:

$$
\phi_{\text {emi }}=\left(A_{\text {referencia }} / A_{\text {muestra }}\right)\left(I_{\text {muestra }} / I_{\text {referencia }}\right) \phi_{\text {emi, referencia }}\left(n_{\text {muestra }} / n_{\text {referencia }}\right)^{2}
$$

donde $I$ es la integral bajo la curva del espectro de emisión, $A$ es la absorbancia de la muestra ó de la referencia a la longitud de onda de excitación y $n$ es el índice de refracción del solvente puro $\left(n_{\mathrm{ACN}}=1,3400 ; n_{\mathrm{DCM}}=1,4200\right.$ y $\left.n_{\mathrm{EtOH}}=1,36143\right)$

Los experimentos de fotólisis resuelta en el tiempo se llevaron a cabo con un equipo de láser flash-fotólisis (FP) como el descripto en el capítulo 1. 
En estos experimentos, pulsos de luz de $351 \mathrm{~nm}$ y duración $\leq 20 \mathrm{~ns}$, fueron generados con un láser de excímero Lambda-Physik SLL-200. La energía del pulso del láser fue atenuada a valores iguales ó menores que $20 \mathrm{~mJ} /$ pulso, absorbiendo parte de la luz del láser con soluciones de $\mathrm{Ni}\left(\mathrm{ClO}_{4}\right)$ de densidades ópticas apropiadas. Los experimentos de fluorescencia resuelta en el tiempo $(\mathrm{FF})$ fueron llevados a cabo en un instrumento para fluorescencia flash (PTI). En este caso, la luz de excitación proviene de un láser de $\mathrm{N}_{2}$ con un ancho de pulso de $0.2 \mathrm{~ns} \mathrm{y} \approx 2 \mathrm{~mJ} /$ pulso. Las soluciones utilizadas en todos los experimentos de fotólisis, fueron deaereadas con corrientes de $\mathrm{N}_{2}$ ultrapuro antes y durante las irradiaciones. Las concentraciones de los fotolitos fueron ajustadas para mantener una concentración homogénea de intermediario fotogenerado dentro del volumen de solución irradiada.

Los espectros diferencia de las especies generadas electroquímicamente fueron obtenidas insitu con un espectrómetro de reflectancia VIS (ver capítulo 1). Básicamente, un espectrómetro, que posee un doble monocromador para obtener baja dispersión de luz, focaliza la radiación sobre el electrodo a un ángulo de $45^{\circ}$. La celda óptica posee ventanas de cuarzo con la orientación adecuada al ángulo de incidencia. El electrodo es un disco de platino cuya superficie es pulida a espejo como se describió anteriormente. El potencial del electrodo y la luz fueron modulados a $11 \mathrm{~Hz}$. Los cambios en la intensidad reflejada, como señal ac $\Delta \mathrm{R}$, fueron detectados con un fotomultiplicador y rectificados por un amplificador de detección sincrónico. El espectro obtenido de esta forma es, a menos que se aclare otra cosa, la diferencia normalizada de la respuesta óptica a un potencial dado y al potencial tomado como referencia, $R_{\text {ref, }}$ es decir, $\Delta R / R_{\text {ref }}=\left(R-R_{\text {ref }}\right) / R_{\text {ref }}$ vs longitud de onda.

Otros detalles de los potenciales electroquímicos medidos se describieron en el capítulo anterior, lo mismo que la obtención de los espectros de absorción UV-visible (capítulo 2, figura 3) 


\section{Espectroscopía de absorción y emisión UV-visible}

El espectro de absorción del aq-2- $\mathrm{CO}_{2}-\mathrm{Re}^{\mathrm{I}}(\mathrm{CO})_{3}(2,2$ 'bipi), tomado en varios solventes, fue mostrado en el capítulo anterior. Los rasgos característicos son la presencia de una banda de absorción de baja energía, $\lambda \approx 400 \mathrm{~nm}$, que fuera asignada a una transición de transferencia de carga $\mathrm{d} \pi\left(\operatorname{Re}^{\mathrm{I}}\right) \rightarrow \pi^{*}\left(2,2^{\prime}\right.$ bipi $)$, que genera el estado excitado $\mathrm{TCML}_{\mathrm{Re} \rightarrow 2,2^{\prime} \text { bipi; }}$ la próxima a mayor energía, $\lambda=292 \mathrm{~nm}$, asignada a una transición $\pi \rightarrow \pi^{*}$ centrada en la 2,2'bipi y otra a longitudes de onda más corta, $\lambda=257 \mathrm{~nm}$, asignada a una transición $\pi \rightarrow \pi^{*}$ centrada en la aq-2- $\mathrm{CO}_{2}$, poblando, estas dos últimas un estado excitado de naturaleza IL.

Otra banda de absorción correspondiente a una transición de transferencia de carga de $\mathrm{d} \pi\left(\mathrm{Re}^{\mathrm{I}}\right)$ $\rightarrow \pi *\left(\right.$ aq- $\left.\mathrm{CO}_{2}\right)$ cabía esperarla a longitudes de onda más largas que las transiciones anteriores, es decir a $\lambda \approx 500 \mathrm{~nm}$. Esta presunción está avalada por la diferencia entre los potenciales de reducción del ligando aq-2- $\mathrm{CO}_{2}$ y del $\mathrm{Re}^{\mathrm{I}}$, como veremos más adelante. La ausencia de esta banda en el espectro es remarcable ya que las transiciones de transferencia de carga resultan, usualmente, en absorciones intensas. Las razones para este fenómeno serán discutidas posteriormente.

La dependencia de los espectros de emisión del complejo de $\mathrm{Re}^{\mathrm{I}}$ con la naturaleza del solvente y con la longitud de onda de excitación, figura 1, se asemeja al comportamiento observado en sistemas relacionados [11]. Por otro lado, la emisión se muestra como una banda ancha sin estructura, con un máximo alrededor de $\lambda \approx 620 \mathrm{~nm}$ semejante a la observada en el $\operatorname{BrRe}^{\mathrm{I}}(\mathrm{CO})_{3}(2,2$ ’bipi). La emisión tiene las características típicas de la correspondiente a un estado excitado ${ }^{3} \mathrm{TCML}_{\mathrm{Re} \rightarrow 2,2}$ 'bipi $[16,23]$. En DCM, cuando cambiamos la longitud de onda de excitación de 350 a $400 \mathrm{~nm}$, la intensidad de la emisión aumenta aunque no se observan cambios en la forma del espectro. Sin embargo, en $\mathrm{ACN}$, se observa un pequeño corrimiento de la emisión hacia energías menores desapareciendo, al excitar con luz de $\lambda=400 \mathrm{~nm}$, un 
componente emisor en $\lambda \approx 520 \mathrm{~nm}$. Por otro lado, no se ha observado efecto con el solvente $\mathrm{y}$ longitud de onda en los espectros de emisión del $\operatorname{BrRe}^{\mathrm{I}}(\mathrm{CO})_{3}\left(2,2^{\prime}\right.$ bipi), como se muestra en la figura 2 junto con el espectro correspondiente al aq-2- $\mathrm{CO}_{2} \mathrm{H}$.

Sobre esta base, entonces, los rasgos principales del espectro pueden ser asociados por emisiones solapadas de dos estados excitados, probablemente IL y ${ }^{3} \mathrm{TCML}_{\mathrm{Re} \rightarrow 2,2^{\prime} \text { bipi. Ningún }}$ rasgo en los espectros de emisión pudo ser asociado a un estado excitado del tipo $\mathrm{TCML}_{\mathrm{Re} \rightarrow \mathrm{aq}-}$ 2- $\mathrm{CO}_{2}$ ya que la emisión de tal estado debería observarse a energías menores por causas que se exponen más adelante 


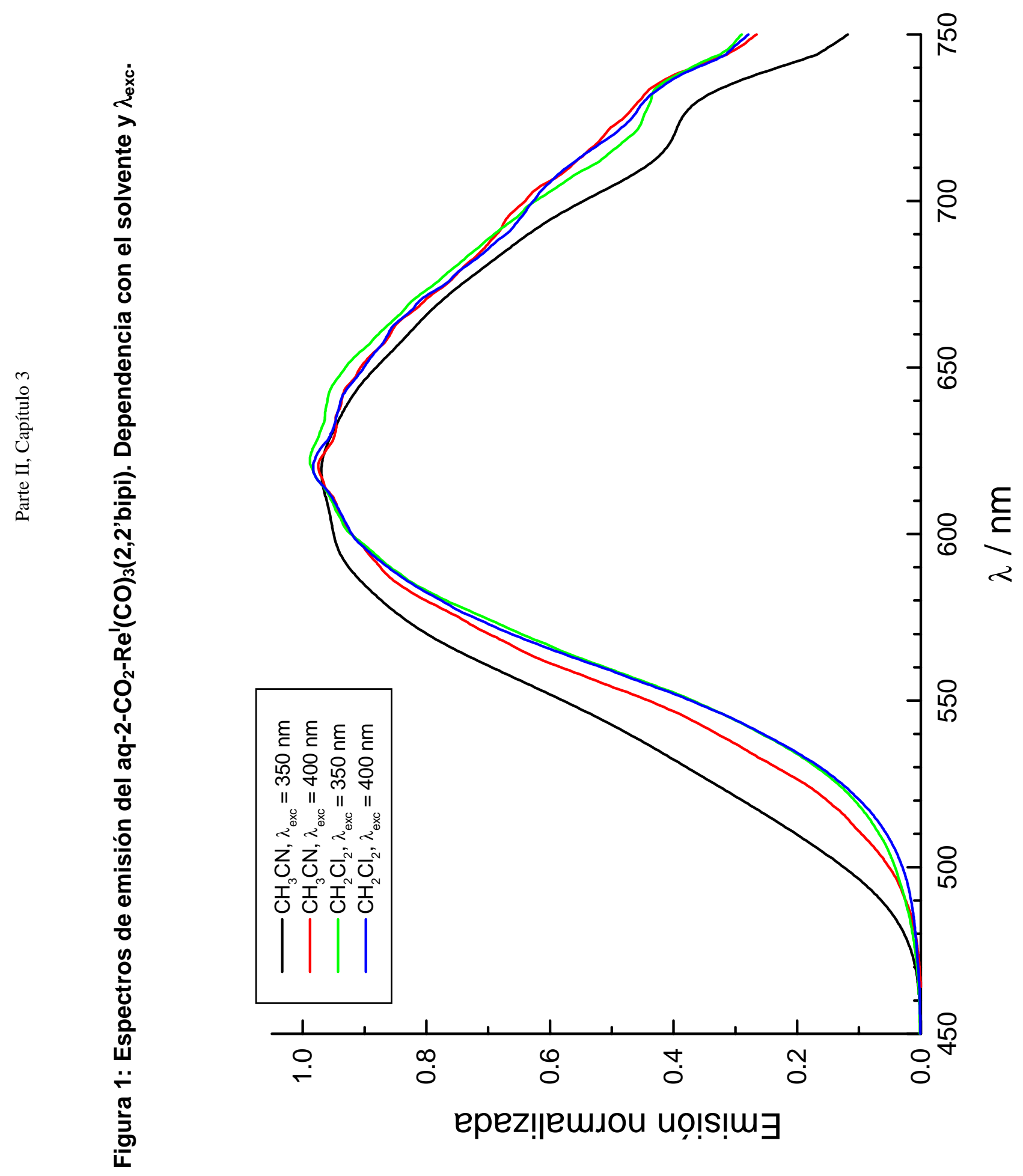




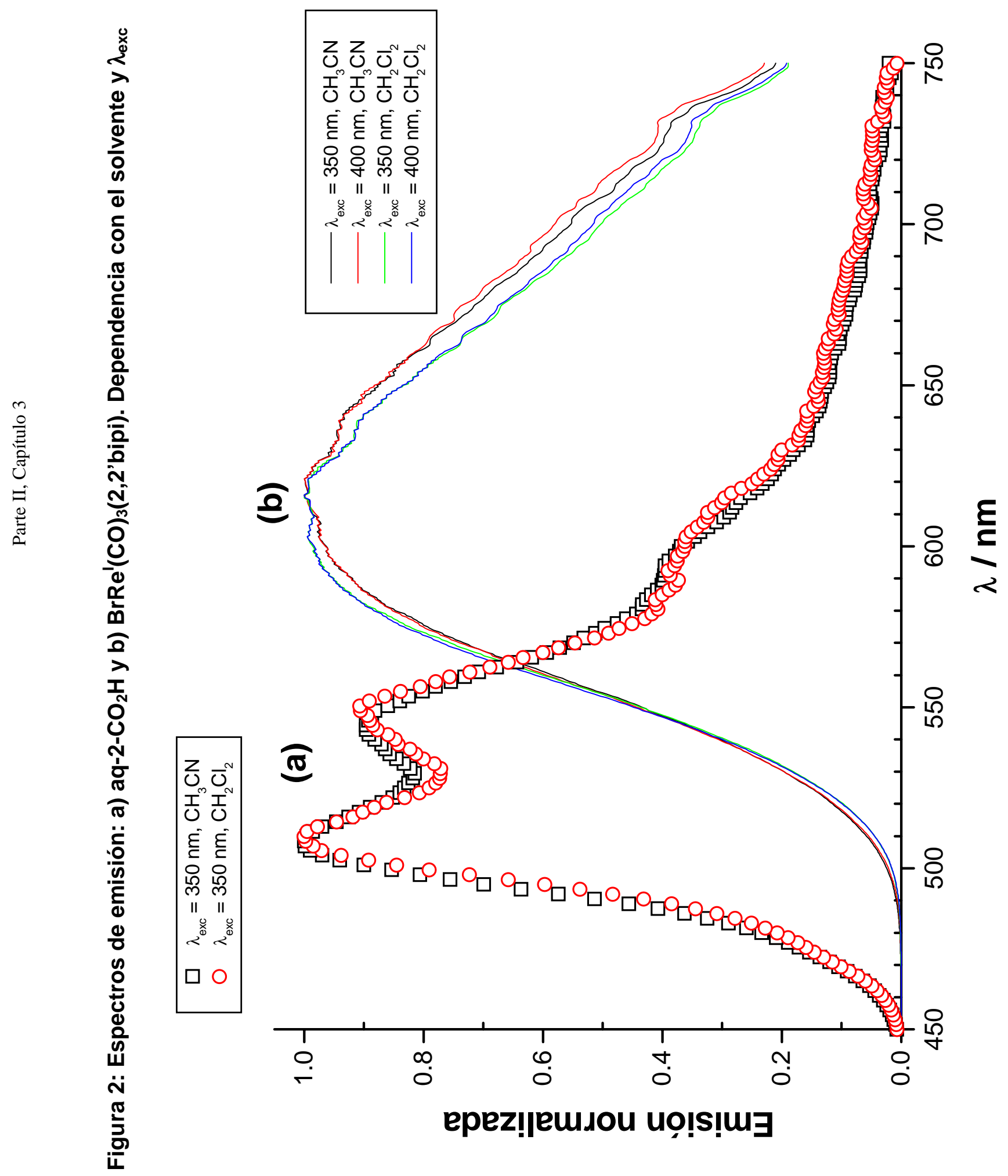




\section{Medidas electroquímicas}

\section{i. $\quad$ Potenciales de media onda}

El procedimiento experimental para la determinación de los $\mathrm{E}_{1 / 2}$ del complejo de $\mathrm{Re}^{\mathrm{I}}$, del precursor y del ligando se ha descripto en el capítulo anterior. Aquí se muestran los resultados obtenidos, que han sido resumidos en la tabla I, junto con otros considerados para la discusión de los datos.

Tabla I: Potenciales electroquímicos vs NHE de los complejo de Re' y ligandos relevantes

\begin{tabular}{|c|c|c|c|c|}
\hline & \multicolumn{4}{|c|}{${ }^{\mathrm{a}} \mathrm{E}_{1 / 2} / \mathrm{V}$ vs NHE } \\
\hline & $+3 \mathrm{e}$ & $+2 \mathrm{e}$ & $+1 \mathrm{e}$ & $-1 e$ \\
\hline$\left[\operatorname{BrRe}^{\mathrm{I}}(\mathrm{CO})_{3}\left(2,2^{\prime} \mathbf{b i p i}^{\prime}\right)\right]^{\mathrm{b}}$ & & $-1,388$ & $-1,105$ & \\
\hline$\left[(\text { pna }) \operatorname{Re}^{\mathrm{I}}(\mathbf{C O})_{3}\left(2,2^{\prime} \mathbf{b i q}\right)\right]^{+c, d}$ & $-0,98$ & $-0,64$ & $-0,49$ & $+1,70$ \\
\hline$\left[\left(4,4^{\prime}{ }^{\prime}{ }^{\prime} i p i\right) \operatorname{Re}^{I}(C O)_{3}\left(2,2^{\prime} b^{\prime}{ }^{\prime}\right)\right]^{+c, d}$ & $-0,99$ & $-0,67$ & $-0,50$ & $+1,44$ \\
\hline$\left[\text { pna-2-CO} 2-\operatorname{Re}^{\mathrm{I}}(\mathrm{CO})_{3}\left(2,2^{\prime} \text { bipi }\right)\right]^{\mathrm{c}, \mathrm{d}}$ & $-1,96$ & $-1,66$ & $-1,09$ & $+1,57$ \\
\hline$\left[\mathrm{aq}-2-\mathrm{CO}_{2}-\operatorname{Re}^{\mathrm{I}}(\mathrm{CO})_{3}\left(2,2^{\prime} \text { 'bipi }\right)\right]^{\mathrm{b}}$ & $-1,174$ & $-1,051$ & $-0,636$ & $+1,612$ \\
\hline $2,2^{\prime} \mathbf{b i q}^{\mathrm{c}, \mathrm{e}}$ & & & $-0,70$ & \\
\hline
\end{tabular}


4,4'bipi ${ }^{c, f}$

pna $^{\mathrm{c}, \mathrm{g}}$

2,2'bipi ${ }^{\text {ch }}$
$-0,49$

$-0,01 \geq \mathrm{E}_{1 / 2} \geq-0,645$

$\mathrm{aq}-2-\mathrm{CO}_{2} \mathrm{H}^{\mathrm{b}}$

$-1,213-0,757$

a. Medidos a temperatura ambiente en $\mathrm{CH}_{3} \mathrm{CN}$ y $0,1 \mathrm{M}$ de $\mathrm{Bu}_{4} \mathrm{NPF}_{6}$

b. Valores experimentales tomados vs el electrodo de referencia $\mathrm{Ag} / \mathrm{ClAg}$

c. Valores experimentales tomados vs el electrodo de referencia SCE

d. Tomados de [11]

e. Tomados de [1]

f. Tomados de [24]

g. Tomados de [25]. Potenciales medidos en solución acuosa para varias concentraciones de ácido, $0 \square \mathrm{ph} \square 6,8$

h. Tomados de [25] para el catión diprotonado. La reducción del catión monoprotonado presenta un valor estimado entre - 0,5 y - 1,0 V del catión diprotonado.

\section{ii. Espectroelectroquímica}

Los experimentos fueron diseñados para la observación de las especies generadas en las reducciones respectivas, involucrando un electrón, de aq-2- $\mathrm{CO}_{2} \mathrm{H}$ y $\operatorname{BrRe}^{\mathrm{I}}(\mathrm{CO})_{3}(2,2$ 'bipi). El espectro in situ de reflectancia diferencial de la figura 3a, fue obtenido de una solución 5,6.10-

${ }^{4} \mathrm{M}$ de aq-2- $\mathrm{CO}_{2} \mathrm{H}$ en $0,1 \mathrm{M}$ de $\mathrm{Bu}_{4} \mathrm{NPF}_{6}$ en acetonitrilo. Para una modulación del potencial entre $\mathrm{E}_{\text {ref }}=-0,66 \mathrm{~V}$ y $\mathrm{E}=-1,16 \mathrm{~V}(\mathrm{vs} \mathrm{Ag} / \mathrm{ClAg})$ corresponde un $\Delta \mathrm{DO} \approx \Delta \mathrm{R} / \mathrm{R}=\left[\mathrm{R}_{-1,16}-\mathrm{R}\right.$. $0,66] / \mathrm{R}_{-0,66}{ }^{6)}$. El espectro de la figura $3 \mathrm{~b}$, fue obtenido de una solución $3,6.10^{-4} \mathrm{M}$ de $\operatorname{BrRe}^{\mathrm{I}}(\mathrm{CO})_{3}\left(2,2^{\prime}\right.$ bipi) en $0,1 \mathrm{M}$ de $\mathrm{Bu}_{4} \mathrm{NPF}_{6}$ en $\mathrm{ACN}$. En este experimento la modulación de potencial fue entre $\mathrm{E}=-1,86 \mathrm{~V}$ y $\mathrm{E}_{\text {ref }}=-1,16 \mathrm{~V}$ (vs $\mathrm{Ag} / \mathrm{ClAg}$ ), por lo tanto, $\Delta \mathrm{DO} \approx \Delta \mathrm{R} / \mathrm{R}=$

\footnotetext{
6) $\Delta \mathrm{DO}=$ cambio de la densidad óptica; $\Delta \mathrm{R} / \mathrm{R}=$ cambio normalizado de la reflectancia
} 
$\left[\mathrm{R}_{-1,86}-\mathrm{R}_{-1,16}\right] / \mathrm{R}_{-1,16}$. El espectro de la figura $3 \mathrm{a}$, fue asignado al radical semiquinona, aq-2$\mathrm{CO}_{2} \mathrm{H}^{\bullet-}$. En la figura 3b, el espectro, tomado a un potencial donde un electrón es adicionado al $\operatorname{BrRe}^{\mathrm{I}}(\mathrm{CO})_{3}\left(2,2^{\prime}\right.$ 'bipi) (Tabla I), puede ser asignado al $\left[\operatorname{BrRe}^{\mathrm{I}}(\mathrm{CO})_{3}\left(2,2^{\prime} \text { bipi }{ }^{\bullet}\right)\right]^{-}$. Un estudio más detallado sobre la espectroelectroquímica del complejo aq-2- $\mathrm{CO}_{2}-\mathrm{Re}(\mathrm{CO})_{3}(2,2$ ’bipi) se describe en el capítulo 4.

Figura 3: Espectros obtenidos en experimentos de espectroelectroquímica con soluciones deaereadas de a) $5,6.10^{-4} \mathrm{M}$ de aq-2- $\mathrm{CO}_{2} \mathrm{H}$ y b) $3,6.10^{-4} \mathrm{M}$ de $\operatorname{BrRe}^{\prime}(\mathrm{CO})_{3}(2,2$ 'bipi) en $\mathrm{ACN}$

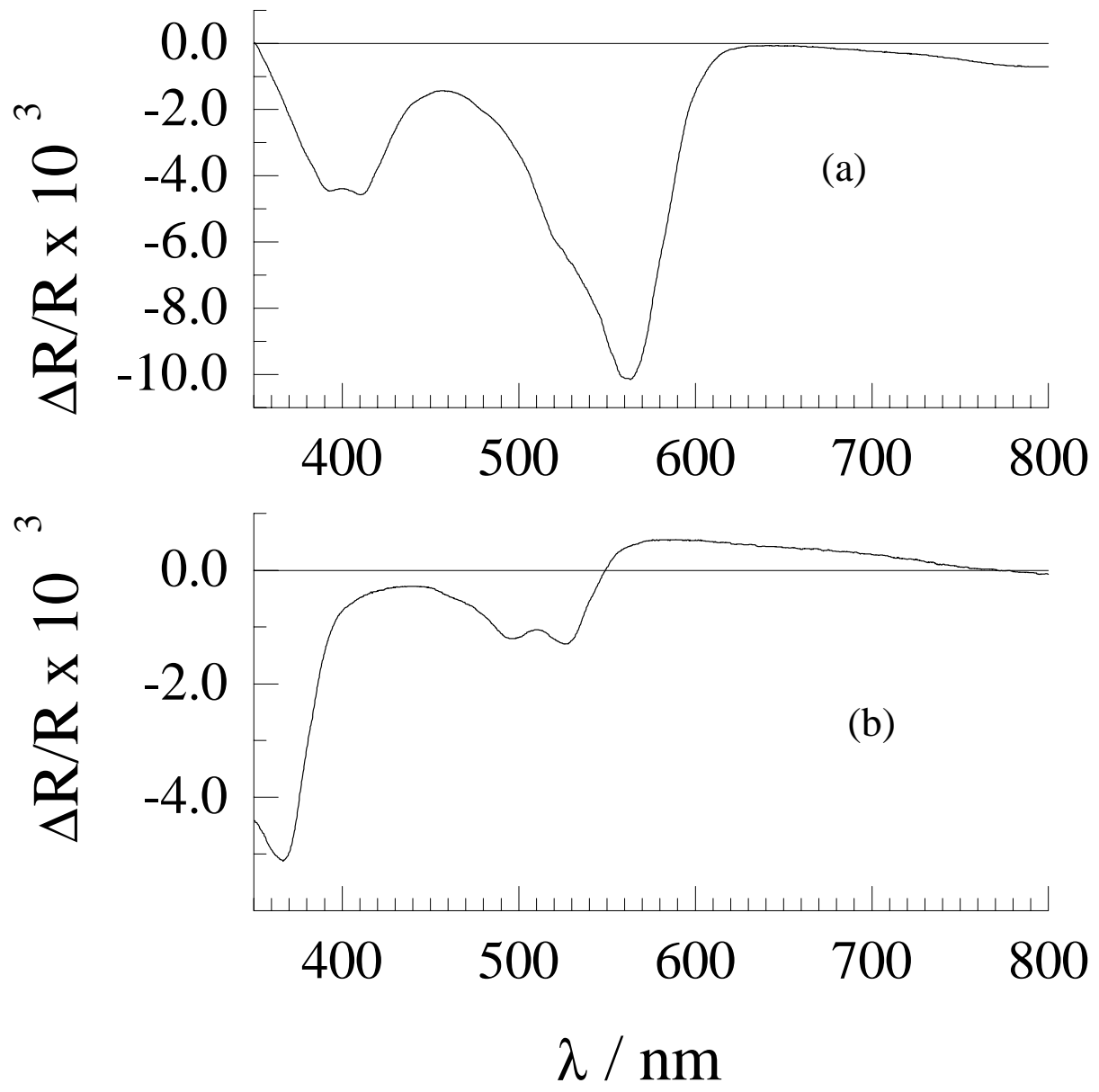


Se verá que estas asignaciones concuerdan bien con los resultados de los experimentos fotoquímicos discutidos a continuación. Además, permiten asignar la reducción de aq-2$\mathrm{CO}_{2}-\mathrm{Re}^{\mathrm{I}}(\mathrm{CO})_{3}(2,2$ 'bipi) a -0,636 V vs NHE (Tabla I) como reducción del ligando aq-2$\mathrm{CO}_{2}^{-}$. En la figura 4 se muestran los espectros del complejo de $\mathrm{Re}^{\mathrm{I}}$ en estudio junto con los del ligando y el precursor para las mismas modulaciones de potencial. En principio, se puede observar que los espectros del aq-2- $\mathrm{CO}_{2}-\mathrm{Re}^{\mathrm{I}}(\mathrm{CO})_{3}\left(2,2^{\prime}\right.$ 'bipi) reducido presenta los rasgos observados en los radicales aq-2- $\mathrm{CO}_{2} \mathrm{H}^{\bullet-}$ y $\left[\mathrm{BrRe}(\mathrm{CO})_{3}\left(2,2^{\prime} \text { bipi }{ }^{\bullet}\right)\right]^{-}$. Más adelante volveremos sobre este tema, relacionando estos espectros con los obtenidos fotoquímicamente. 


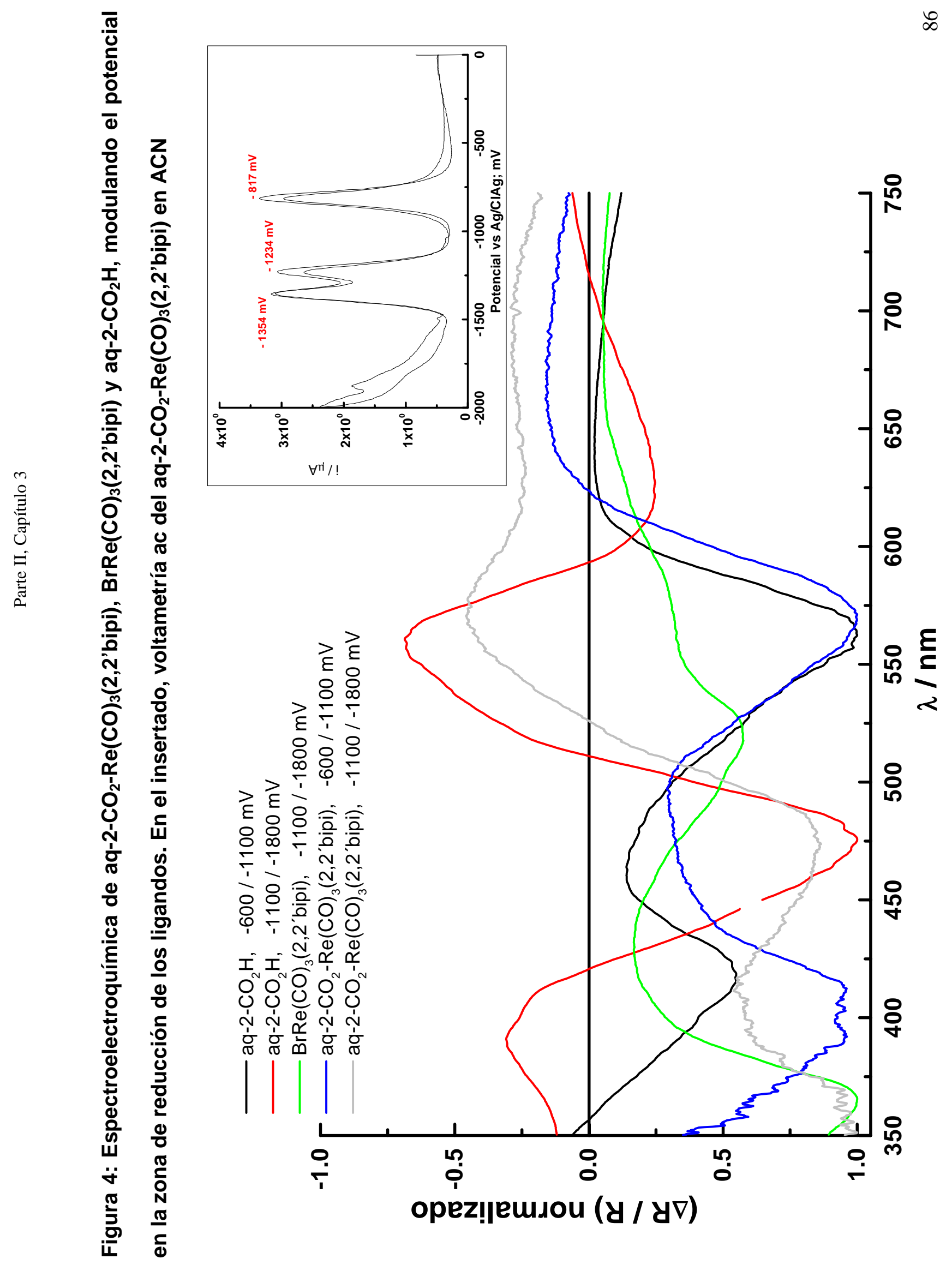




\section{Espectroscopía resuelta en el tiempo}

\section{i. $\quad$ Fotólisis de aq-2- $\mathrm{CO}_{2} \mathrm{H}$}

El espectro generado irradiando soluciones de aq-2- $\mathrm{CO}_{2} \mathrm{H}$, con pulsos de luz de $351 \mathrm{~nm}$, depende fuertemente del solvente, figura 5. Esta dependencia con el solvente es similar a la observada para los tripletes de otros derivados de antraquinonas [26-28]. En la figura 5a se puede ver el espectro de la especie transitoria (transiente) obtenido al fotolizar una solución $1,0.10^{-4} \mathrm{M}$ de aq-2- $\mathrm{CO}_{2} \mathrm{H}$ en $\mathrm{ACN}$ libre de oxígeno. Las trazas del decaimiento de la absorción entre las longitudes de onda 370 y 700 nm fueron ajustadas muy bien como una función exponencial simple:

$$
\mathrm{A}=\mathrm{A}_{0} \mathrm{e}^{-\mathrm{t} / \tau}
$$

donde $\tau$ es el tiempo de vida del transiente y cuyo valor fue de $3,5 \mu$ s.

En experimentos de fluorescencia resuelta en el tiempo realizados irradiando soluciones similares con $\lambda_{\text {exc }}=337 \mathrm{~nm}$ y observando la emisión a $\lambda_{\text {obs }}=510 \mathrm{~nm}$, los decaimientos pudieron ajustarse bien con un solo exponencial $(\tau=(7,5 \pm 0,8) \mu s)$. A diferencia de los experimentos realizados en $\mathrm{ACN}$, una función de tipo biexponencial fue la que permitió obtener el mejor ajuste de las trazas obtenidas para el decaimiento del transiente de absorción en $\mathrm{MeOH}$, figura 5b. Los tiempo de vida obtenidos en este caso fueron, $\tau_{1}=(932 \pm 5) \mathrm{ns}$ y $\tau_{2}$ $=(5,1 \pm 0,3) \mu \mathrm{s}$. Las características del espectro y la dependencia de éste con el solvente, correlacionan con la observada para los tripletes de la antraquinona y serán respectivamente denominados ${ }^{\mathrm{T}} \mathrm{aq}-2-\mathrm{CO}_{2} \mathrm{H}$ y ${ }^{\mathrm{T}} \mathrm{aq}-2-\mathrm{CO}_{2} \mathrm{H}^{\prime}$. Las siguientes ecuaciones 1-3 esquematizan la formación y decaimiento de estos tripletes: 


$$
\begin{aligned}
& \mathrm{aq}-2-\mathrm{CO}_{2} \mathrm{H}+h v \longrightarrow{ }^{\mathrm{T}} \mathrm{aq}-2-\mathrm{CO}_{2} \mathrm{H}+{ }^{\mathrm{T}} \mathrm{aq}-2-\mathrm{CO}_{2} \mathrm{H}^{\prime} \\
& { }^{\mathrm{T}} \mathrm{aq}-2-\mathrm{CO}_{2} \mathrm{H} \longrightarrow \mathrm{aq}-2-\mathrm{CO}_{2} \mathrm{H}+h v^{\prime}, \quad \tau_{1}=932 \mathrm{~ns} \\
& { }^{\mathrm{T}} \mathrm{aq}-2-\mathrm{CO}_{2} \mathrm{H}^{\prime} \longrightarrow \mathrm{aq}-2-\mathrm{CO}_{2} \mathrm{H}+h v^{\prime \prime}, \quad \tau_{2}=5 \mu \mathrm{s}
\end{aligned}
$$

Las especies ${ }^{\mathrm{T}} \mathrm{aq}-2-\mathrm{CO}_{2} \mathrm{H}$ y ${ }^{\mathrm{T}}$ aq-2- $\mathrm{CO}_{2} \mathrm{H}^{\prime}$ en las ecuaciones 2 y 3 pueden ser las mismas especies solvatadas que han sido observadas en los procesos fotofísicos de varias antraquinonas [26]. Esta propuesta provee una buena herramienta para racionalizar la fotofísica de aq-2- $\mathrm{CO}_{2} \mathrm{H}$ en DCM. En efecto, una luminiscencia de vida muy corta con un $\tau \sim$ 10 ns fue observado en experimentos de FF excitando a $337 \mathrm{~nm}$ soluciones deaereadas de aq2- $\mathrm{CO}_{2} \mathrm{H}$ en DCM. La reacción entre los tripletes de la aq-2- $\mathrm{CO}_{2} \mathrm{H}$ y el solvente, ó un decaimiento de la luminiscencia asistida por el solvente, podría ser la causa de la corta vida de esta luminiscencia. Una comparación de los rendimientos cuánticos de emisión en DCM $(\phi=$ $\left.(8,6 \pm 0,5) \cdot 10^{-4}\right)$ y $\mathrm{ACN}\left(\phi=(1,7 \pm 0,2) \cdot 10^{-3}\right)$ y los correspondientes tiempos de vida de la emisión avalan la proposición de participación del solvente (tabla II). La baja solubilidad del aq-2- $\mathrm{CO}_{2} \mathrm{H}$ en DCM impidió la aplicación de espectroscopía resuelta en el tiempo en la observación de los espectros de absorción de los estados excitados, en este solvente. 
Figura 5: Espectro de la especie transitoria generada irradiando, con pulsos de luz de $351 \mathrm{~nm}$, soluciones deaereadas de aq-2- $\mathrm{CO}_{2} \mathrm{H} 1,0.10^{-4} \mathrm{M}$ a) en $\mathrm{ACN}$ y b) en $\mathrm{MeOH}$
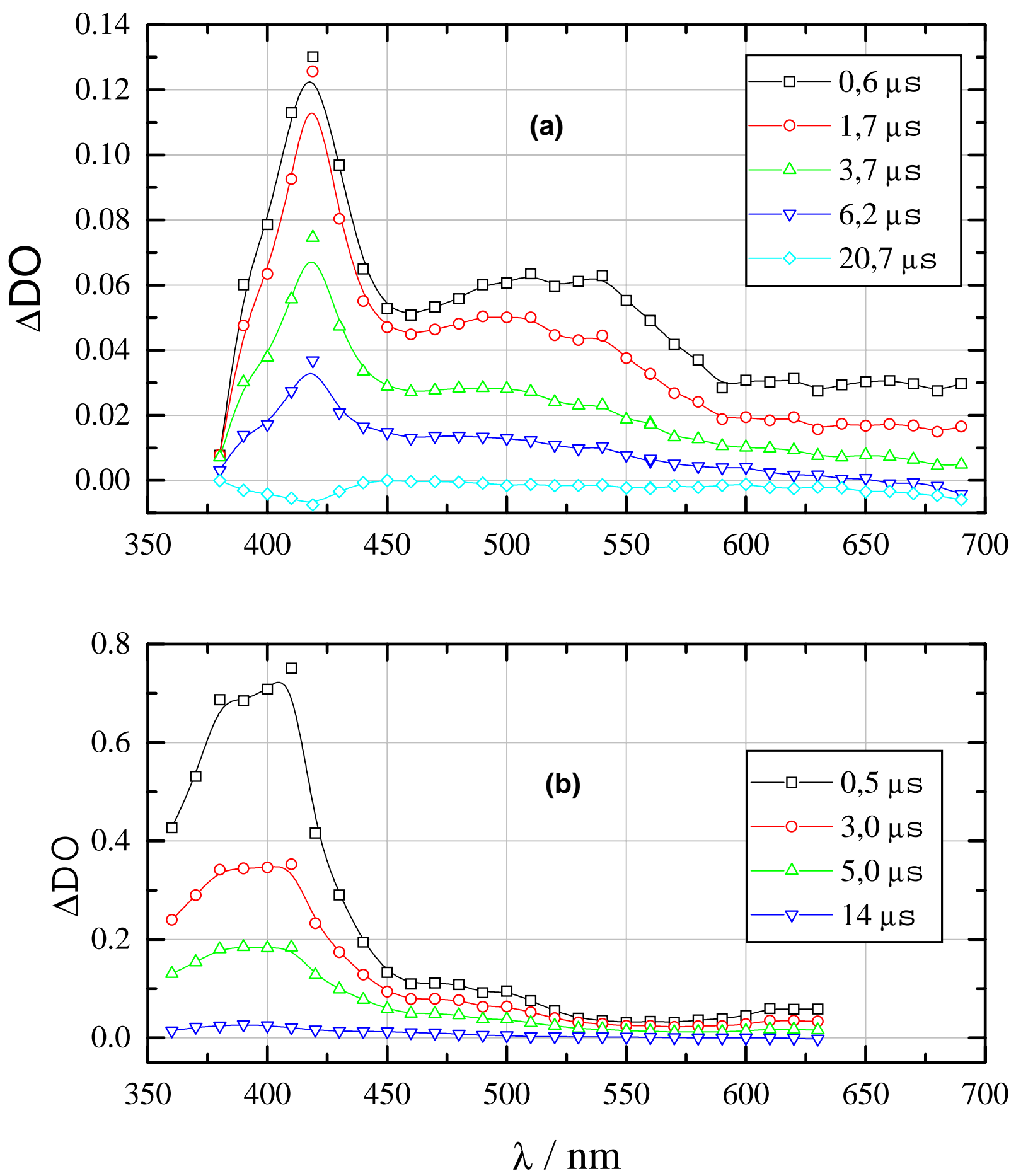
El radical de la semiquinona aq-2- $\mathrm{CO}_{2} \mathrm{H}^{\bullet-}$ fue obtenido utilizando la técnica de láser flash fotólisis, y su espectro de absorción usado para la caracterización de las especies generadas en las reacciones fotoredox del aq-2- $\mathrm{CO}_{2}-\mathrm{Re}^{\mathrm{I}}(\mathrm{CO})_{3}(2,2$ ' bipi). Para obtener el espectro del aq-2$\mathrm{CO}_{2} \mathrm{H}^{\bullet-}$, los tripletes del aq-2- $\mathrm{CO}_{2} \mathrm{H}$ fueron reducidos con aminas donores de electrones como la trietilamina (TEA) y la trietanolamina (TEOA). El espectro de la especie transitoria, se generó irradiando con pulsos de luz de $351 \mathrm{~nm}$ una solución deaereada de concentración $1,0.10^{-4} \mathrm{M}$ en ACN y que además contenía $1,0.10^{-3} \mathrm{M}$ de TEA, figura 6 . Este transiente decae por un proceso que sigue una cinética de primer orden con un $\tau=(5,1 \pm 0,3) \mu$ s. El espectro puede ser asignado al radical de la semiquinona aq-2- $\mathrm{CO}_{2} \mathrm{H}^{\bullet-}$ por comparación con el espectro generado en los experimentos de espectroelectroquímica y por los espectros de radicales de semiquinonas relacionadas que se encuentran en la literatura $[26,29,30]$. Estas observaciones sugieren que los tripletes generados por los procesos descriptos en las ecuaciones 1 a 3 reaccionan con TEA y TEOA por transferencia de electrones. Las reacciones con TEA se ilustran en las ecuaciones 4 y 5.

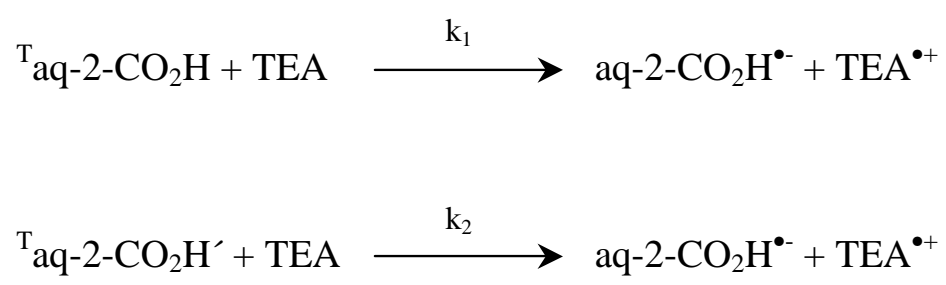

Un valor para la constante de quenching global, $\mathrm{k}_{\mathrm{Q}}=\mathrm{k}_{1}+\mathrm{k}_{2}=5.10^{9} \mathrm{M}^{-1} \mathrm{~s}^{-1}$, fue calculado de la curva de crecimiento monoexponencial del radical aq-2- $\mathrm{CO}_{2} \mathrm{H}^{\bullet-}$ con un $\tau$ de $(210 \pm 10) \mathrm{ns}$ (ver el insertado de la figura 6). Este valor de $\mathrm{k}_{\mathrm{Q}}$ se obtiene dividiendo la inversa del $\tau$ por el valor de la [TEA], $1.10^{-3} \mathrm{M}$, por resolución de la ecuación cinética de pseudo-primer orden donde se cumple que $\tau=1 / \mathrm{k}$ siendo $\mathrm{k}=\mathrm{k}_{\mathrm{Q}}[\mathrm{TEA}]$ 
Por otro lado, se llevaron a cabo experimentos de fluorescencia resuelta en el tiempo $\left(\lambda_{\text {exc }}=\right.$ $337 \mathrm{~nm}$ ) en soluciones deaereadas de aq-2- $\mathrm{CO}_{2} \mathrm{H}$ en $\mathrm{ACN}$ conteniendo diferentes concentraciones de TEOA $\left(0 \square[\right.$ TEOA $\left.] \square 6.10^{-5} \mathrm{M}\right)$. El quenching de la luminiscencia de aq-2$\mathrm{CO}_{2} \mathrm{H}$ por TEOA fue similar al descripto para TEA en las ecuaciones 2 a 5 . Las trazas del osciloscopio mostraron que el decaimiento de la fluorescencia puede ser ajustado con una función biexponencial con $\tau$ 's que dependían de la [TEOA] pero eran independientes de la $\lambda_{\text {obs. }}$ Las constantes de quenching $\mathrm{k}_{1}=(1,7 \pm 0,1) \cdot 10^{10} \mathrm{M}^{-1} \mathrm{~s}^{-1} \mathrm{y} \mathrm{k}_{2}=(9,2 \pm 0,2) \cdot 10^{9} \mathrm{M}^{-1} \mathrm{~s}^{-1}$ fueron calculados de las representaciones de $\tau^{-1}$ versus [TEOA], figura 7a, que corresponden a las siguientes expresiones:

$$
\tau_{1}^{-1}={ }_{1} \tau_{0}^{-1}+\mathrm{k}_{1}[\text { TEOA }] \quad \mathrm{y} \quad \tau_{2}^{-1}={ }_{2} \tau_{0}^{-1}+\mathrm{k}_{2}[\text { TEOA }]
$$

donde $\tau_{\mathrm{n}}^{-1}$ son las inversas de los $\tau_{1}$ y $\tau_{2}$ a cada [TEOA] $\mathrm{y}_{\mathrm{n}} \tau_{0}{ }^{-1}$ son las inversas de los $\tau_{1} \mathrm{y} \tau_{2}$ en ausencia de TEOA.

En medidas de emisión en estado estacionario irradiando a $350 \mathrm{~nm}$, se pudo calcular la constante de Stern-Volmer, $\mathrm{K}_{\mathrm{SV}}=(8,8 \pm 0,3) \cdot 10^{4} \mathrm{M}^{-1}$, de la pendiente del gráfico de $\mathrm{I}_{0} / \mathrm{I}$ versus [TEOA], figura 7b, que corresponde a las siguiente expresión [19]:

$$
\mathrm{I}_{0} / \mathrm{I}=1+\mathrm{K}_{\mathrm{sv}}[\mathrm{TEOA}] \quad \text { donde } \mathrm{K}_{\mathrm{sv}}=\mathrm{k}_{\mathrm{q}} \tau
$$

Este es el mismo valor que se obtiene calculado con las constantes de velocidad de las medidas resueltas en el tiempo, considerando que:

$$
\mathrm{K}_{\mathrm{sv}}=\mathrm{k}_{\mathrm{q}} \tau=\mathrm{k}_{1} \tau_{1}+\mathrm{k}_{2} \tau_{2}=8,8 \cdot 10^{4} \mathrm{M}^{-1}
$$


Figura 6: Espectro del transiente del radical semiquinona generado irradiando con pulsos de luz de $351 \mathrm{~nm}$ soluciones deaereadas de aq-2- $\mathrm{CO}_{2} \mathrm{H} 1,0.10^{-4} \mathrm{M}$ en ACN conteniendo $1,0.10^{-3} \mathrm{M}$ de TEA. Los tiempos de retardo con respecto al pulso del láser son (L) $2,5 \mu \mathrm{s}$; ( ) $5 \mu \mathrm{s}$; ( ) $10 \mu \mathrm{s}$; ( ) $15 \mu \mathrm{s}$. En el insertado se muestra el crecimiento del radical semiquinona a $\lambda_{\text {obs }}=580 \mathrm{~nm}$

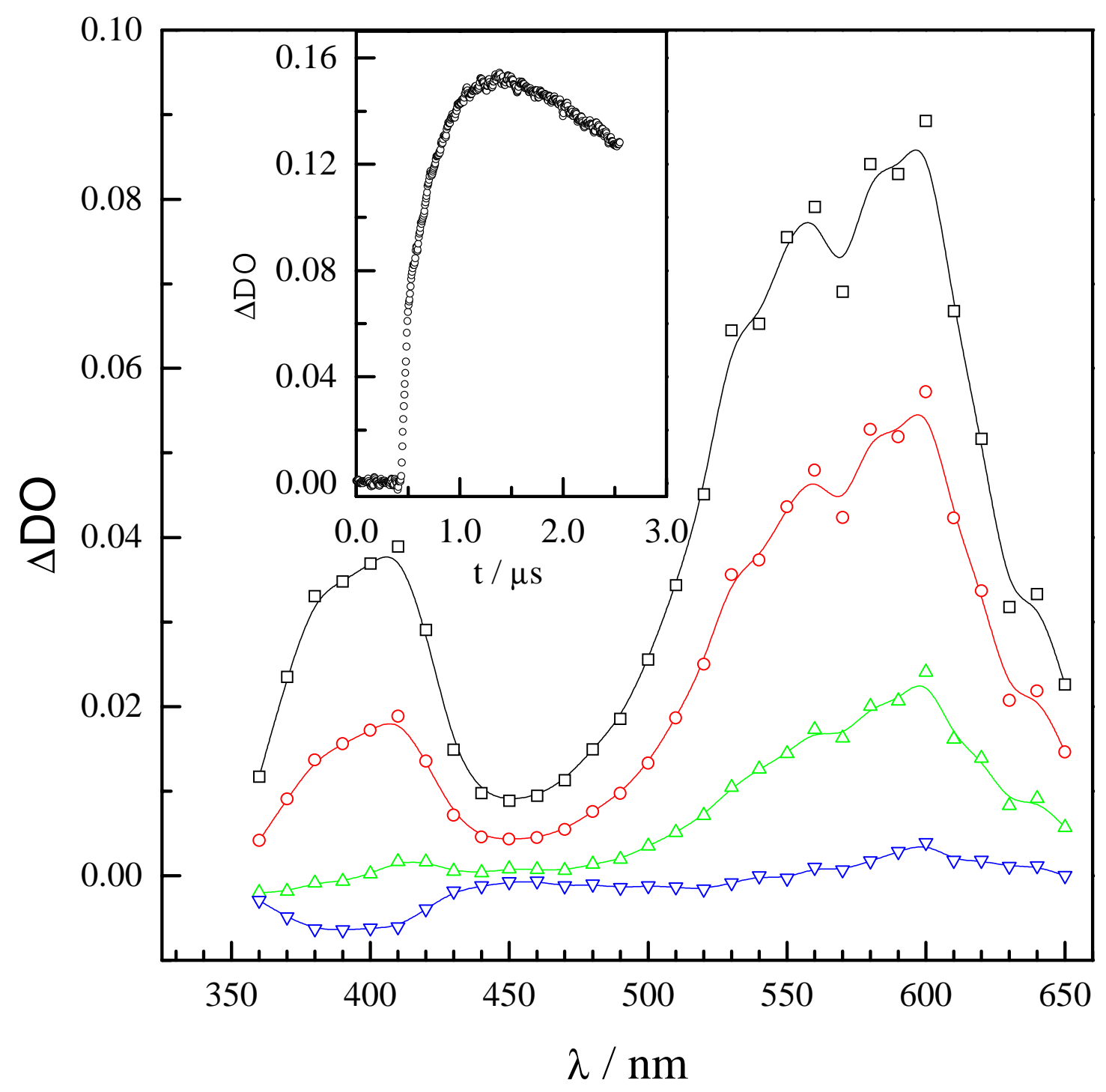


Figura 7: Quenching por TEOA de la luminiscencia de soluciones deaereadas de aq-2- $\mathrm{CO}_{2} \mathrm{H}$ en $\mathrm{ACN}$. a) Experimentos resueltos en el tiempo irradiando con pulsos de luz de $337 \mathrm{~nm}$. b) Experimentos en estado estacionario, excitando a $350 \mathrm{~nm}$.

(a)
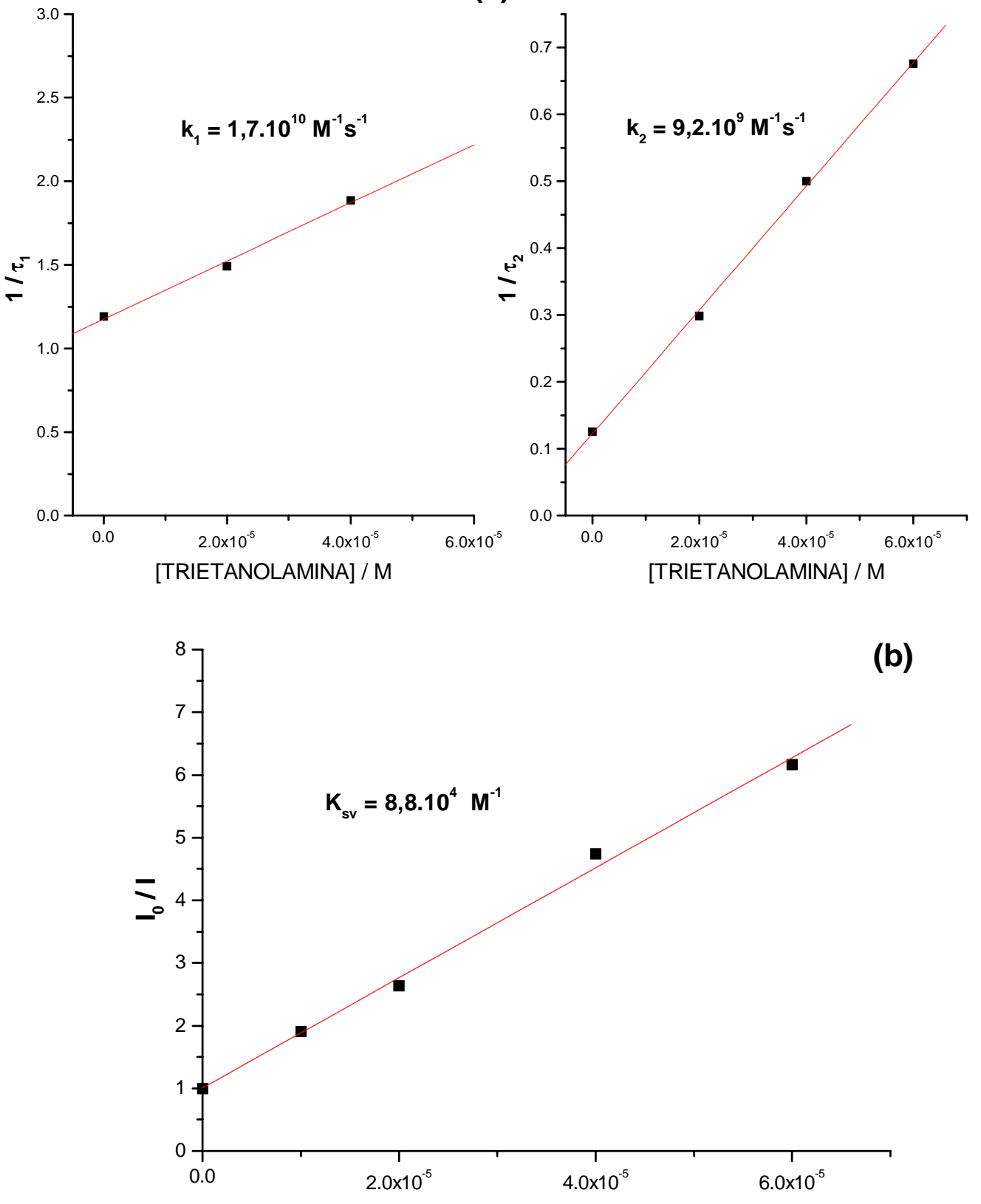

[TRIETANOLAMINA] / M 


\section{ii. $\quad$ Fotólisis de aq-2-CO $-\mathrm{Ce}_{2}(\mathrm{CO})_{3}\left(2,2^{\prime}\right.$ 'bipi)}

La irradiación con pulsos de luz de $351 \mathrm{~nm}$ de soluciones deaereadas de concentración $2.10^{-4}$ $\mathrm{M}$ de aq-2- $\mathrm{CO}_{2}-\mathrm{Re}^{\mathrm{I}}(\mathrm{CO})_{3}\left(2,2^{\prime}\right.$ 'bipi) en $\mathrm{ACN}$, produjeron un transitorio cuyo espectro puede

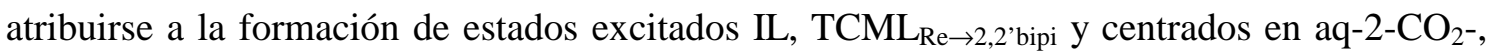
ecuación 6.

$$
\begin{aligned}
& {\left[\mathrm{aq}^{2}-\mathrm{CO}_{2}-\mathrm{Re}^{\mathrm{I}}(\mathrm{CO})_{3}\left(2,2^{\prime} \text { bipi }\right)\right]+h v \longrightarrow }\left\{\mathrm{IL}+\mathrm{TCML}_{\mathrm{Re} \rightarrow 2,2^{\prime} \text { bipi }}+\right. \\
&\left.+\left[{ }^{\mathrm{T}} \mathrm{aq}-2-\mathrm{CO}_{2}-\operatorname{Re}^{\mathrm{I}}(\mathrm{CO})_{3}\left(2,2^{\prime} \text { bipi }\right)\right]+\left[{ }^{\mathrm{T}} \mathrm{aq}-2-\mathrm{CO}_{2}{ }^{\prime}-\operatorname{Re}^{\mathrm{I}}(\mathrm{CO})_{3}\left(2,2^{\prime} \text { bipi }\right)\right]\right\}
\end{aligned}
$$

El espectro de este transitorio decae en tres pasos con dependencia exponencial, $\exp \left(-\mathrm{t} / \tau_{\mathrm{i}}\right)$ donde $\tau_{\mathrm{i}}$ con $\mathrm{i}=1,2,3$ son las vidas medias de los tres decaimientos. Los primeros dos pasos fueron similares a los observados previamente en la fotofísica de pna-2- $\mathrm{CO}_{2^{-}}$ $\operatorname{Re}^{\mathrm{I}}(\mathrm{CO})_{3}(2,2$ 'bipi) y asignados al decaimiento de un estado excitado IL centrado en la 2,2`bipi y otro de transferencia de carga $\operatorname{Re}^{\mathrm{I}}$ a $2,2^{\prime}$ bipi, $\mathrm{TCML}_{\mathrm{Re} \rightarrow 2,2^{\prime} \text { bipi }}$ [11]. En el complejo de $\operatorname{Re}^{\mathrm{I}}$ en estudio el primer paso, $\tau_{1} \approx(41 \pm 5) \mathrm{ns}$, fue asignado al decaimiento del estado excitado intraligante IL, figura 8. Un segundo paso en el decaimiento del transiente con

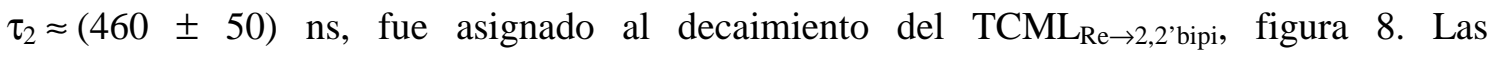
observaciones experimentales sugieren que excitando con $\lambda_{\text {exc }} \square 350 \mathrm{~nm}$, ecuación 6 , se pueblan los estados excitados IL y $\mathrm{TCML}_{\mathrm{Re} \rightarrow 2,2^{\prime} \text { bipi, }}$ los cuales decaen vía ecuaciones 7 y 8 . La

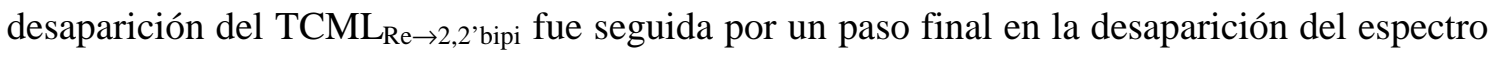
del transiente con un $\tau_{3} \approx(4,9 \pm 0,5) \mu$ s. Este paso final puede ser asignado al decaimiento del ${ }^{\mathrm{T}}$ aq-2- $\mathrm{CO}_{2}{ }^{\prime}$ coordinado al $\mathrm{Re}^{\mathrm{I}}$ por comparación con la fotofísica del aq-2- $\mathrm{CO}_{2} \mathrm{H}$ libre en $\mathrm{ACN}$, ecuación 9. 
IL

$$
\left[\mathrm{aq}-2-\mathrm{CO}_{2}-\mathrm{Re}^{\mathrm{I}}(\mathrm{CO})_{3}\left(2,2^{\prime} \text { bipi }\right)\right]+h v^{\prime} ; \tau_{1}=41 \mathrm{~ns}
$$

TCML $_{\operatorname{Re} \rightarrow 2,2 ' \text { bipi }}$

$$
\longrightarrow \quad\left[\mathrm{aq}-2-\mathrm{CO}_{2}-\mathrm{Re}^{\mathrm{I}}(\mathrm{CO})_{3}\left(2,2^{\prime} \text { bipi }\right)\right]+h v^{\prime \prime} ; \tau_{2}=460 \mathrm{~ns}
$$

$$
\left[{ }^{\mathrm{T}} \text { aq-2- } \mathrm{CO}_{2}{ }^{\prime}-\mathrm{Re}^{\mathrm{I}}(\mathrm{CO})_{3}\left(2,2^{\prime} \text { bipi }\right)\right] \rightarrow\left[\text { aq-2- } \mathrm{CO}_{2}-\operatorname{Re}^{\mathrm{I}}(\mathrm{CO})_{3}\left(2,2^{\prime} \text { bipi }\right)\right]+h v^{\prime \prime \prime} ; \tau_{3}=4,9 \mu \mathrm{s}
$$

Figura 8: Representación logarítmica de los espectros de los transientes registrados con diferentes retardos después del pulso de luz de $351 \mathrm{~nm}$. Los experimentos se realizaron sobre soluciones deaereadas de aq-2- $\mathrm{CO}_{2}$ $\operatorname{Re}^{\prime}(\mathrm{CO})_{3}\left(2,2\right.$ 'bipi) de concentración $2,0.10^{-4} \mathrm{M}$ en ACN

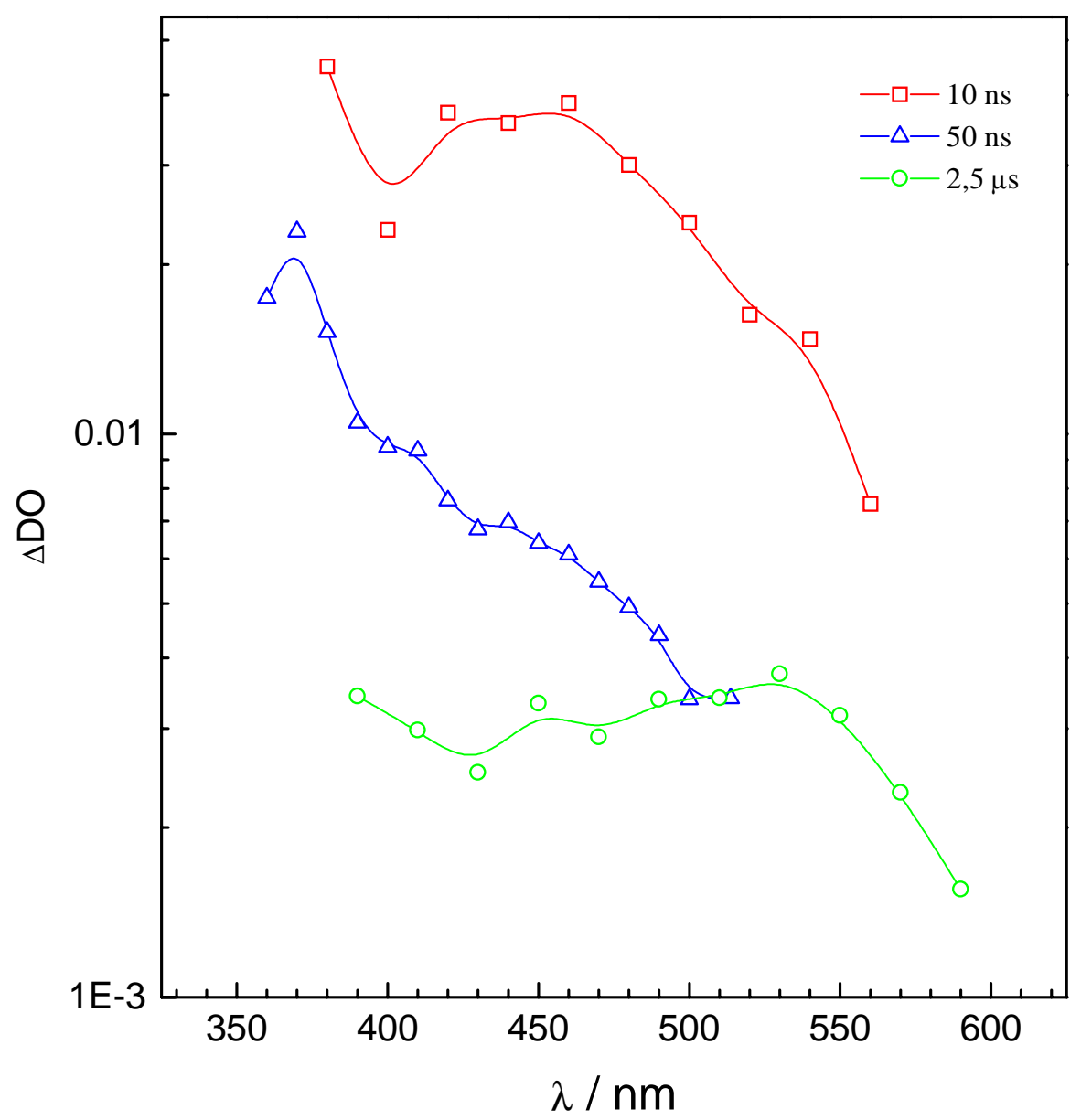


Teniendo en cuenta que los solventes halocarbonados son considerablemente más reactivos que el acetonitrilo, también se investigó el foto-comportamiento del complejo de $\operatorname{Re}^{\mathrm{I}}$ en diclorometano.

Cuando soluciones deaereadas de aq-2- $\mathrm{CO}_{2}-\mathrm{Re}^{\mathrm{I}}(\mathrm{CO})_{3}\left(2,2^{\prime}\right.$ 'bipi), $2.010^{-4} \mathrm{M}$, en diclorometano, son irradiadas con pulsos de luz láser de $351 \mathrm{~nm}$, las especies transitorias que se generan son diferentes a las observadas en acetonitrilo. Uno de los espectros mostrados en la figura 9a es obtenido por extrapolación al comienzo del pulso del láser siendo, además, una convolución (mezcla) de dos espectros. Uno de los componentes del espectro transitorio fue deconvolucionado por medio del espectro registrado con retardos, respecto al disparo del láser, de 20 y 70 ns. El espectro deconvolucionado es de vida corta y se desvanece inmediatamente después que el pulso del láser se ha extinguido, es decir $\mathrm{t} \approx 30 \mathrm{~ns}$. Los rasgos del espectro de vida corta fueron similares a aquellos hallados en el espectro del triplete de la aq-2- $\mathrm{CO}_{2}$ visto en los experimentos de fotólisis pulsada realizados con el ligando libre, figura 5a, y con el complejo en acetonitrilo, figura 8. Es decir, esto se debe a la contribución que al espectro transitorio hacen las especies [ ${ }^{\mathrm{T}} \mathrm{aq}-2-\mathrm{CO}_{2}-\mathrm{Re}^{\mathrm{I}}(\mathrm{CO})_{3}\left(2,2^{\prime}\right.$ bipi $\left.)\right]$

El espectro de las especies transitorias de vida larga exhibe rasgos que fueron previamente observados en el espectro del estado excitado $\mathrm{TCML}_{\mathrm{Re} \rightarrow 2,2^{2} \text { bipi }}$ de los complejos que contienen el grupo cromóforo $-\operatorname{Re}^{\mathrm{I}}(\mathrm{CO})_{3}(2,2$ ’ bipi).

Por otra parte, se compararon las cinéticas de los decaimientos del espectro de absorción de las especies transitorias observadas en los experimentos de FP, con las obtenidas para la extinción de la fluorescencia en experimentos de FF, realizados con soluciones deaereadas de aq-2- $\mathrm{CO}_{2}-\mathrm{Re}^{\mathrm{I}}(\mathrm{CO})_{3}\left(2,2^{\prime}\right.$ bipi $) 10^{-4} \mathrm{M}$ en DCM. Estos decaimientos fueron ambos de primer orden y tienen casi el mismo tiempo de vida, es decir, $\tau=(110 \pm 10)$ ns para el decaimiento de la luminiscencia y $\tau=(116 \pm 20)$ ns para el decaimiento del espectro transitorio, figura 10a. Sobre esta base experimental y comparando con los resultados obtenidos con otros 
complejos de $\operatorname{Re}^{\mathrm{I}}$ relacionados, el componente lento del decaimiento de absorción del espectro transitorio en DCM fue asignado al decaimiento del estado excitado luminiscente $\mathrm{TCML}_{\mathrm{Re} \rightarrow 2,2^{\prime} \text { 'bipi }}$ del aq-2-CO $\mathrm{CO}_{2}-\mathrm{Re}^{\mathrm{I}}(\mathrm{CO})_{3}(2,2$ 'bipi $)$.

En estos experimentos se observa, además, que el decaimiento del espectro del estado excitado $\mathrm{TCML}_{\mathrm{Re} \rightarrow 2,2^{\prime} \text { bipi }}$ no retorna a la densidad óptica de la línea de base. En realidad, la densidad óptica decae hacia la línea de base a través de un proceso cinéticamente lento de primer orden. Para este proceso el decaimiento exponencial del espectro condujo a la obtención de un tiempo de vida $\tau=(3,7 \pm 0,4) \mu \mathrm{s}$, figura 10d. Este espectro transitorio, figura $9 \mathrm{~b}$, exhibe rasgos diferentes a los vistos en el espectro del radical aq-2- $\mathrm{CO}_{2} \mathrm{H}^{\bullet}$. Por el contrario, recuerda estrechamente al espectro del radical $2,2^{\prime}$ bipi ${ }^{\bullet-}$ coordinado que se produce en el quenching reductivo del estado excitado debido a donores de electrones. Que el espectro del transitorio llegue hasta la línea de base sugiere una escasa foto-descomposición del complejo de $\operatorname{Re}^{\mathrm{I}}$ y que el proceso redox tiene un rendimiento cuántico menor que $10^{-4}$ (estimado teniendo en cuenta la concentración de transiente generada en cada disparo del láser y los límites de detección del sistema).

Dados estos resultados se investigó también la posibilidad de la descomposición fotoquímica del aq-2- $\mathrm{CO}_{2}-\mathrm{Re}^{\mathrm{I}}(\mathrm{CO})_{3}(2,2$ 'bipi) fotolizando el complejo en forma estacionaria. Para ello, se irradiaron soluciones deaereadas del complejo en estudio $3,0.10^{-4} \mathrm{M}$ en DCM, excitando con luz de $350 \mathrm{~nm}\left(\mathrm{I}_{0}=6,5 \cdot 10^{-4}\right.$ Einstein $\left.\mathrm{l}^{-1} \mathrm{~min}^{-1}\right)$ por períodos menores ó iguales a $60 \mathrm{~min}$. En plena concordancia con lo observado en los experimentos de fotólisis de pulso, la fotólisis estacionaria no produce cambios permanentes en el espectro de absorción UV-visible de la solución. Por otra parte, tampoco se detectó la presencia del anión $\mathrm{Cl}^{-}$al finalizar la irradiación, como se podría esperar proveniente de la posible descomposición reductiva del solvente; aunque, de ocurrir esta descomposición, el límite de detección del análisis de $\mathrm{Cl}^{-}$ sugiere que el rendimiento cuántico debe ser igual ó menor que $10^{-3}$. 
Figura 9: Espectros de las especies transitorias generadas irradiando con pulsos de luz de $351 \mathrm{~nm}$, soluciones deaereadas de aq-2-CO $-\mathrm{Re}^{\prime}(\mathrm{CO})_{3}\left(2,2^{\prime}\right.$ bipi) $1,0.10^{-4}$ en DCM tomados con retardos de: a) 0,50 y $100 \mathrm{~ns}$ y b) 1, 3, 5, 10 y 20 $\boldsymbol{\mu s}$.
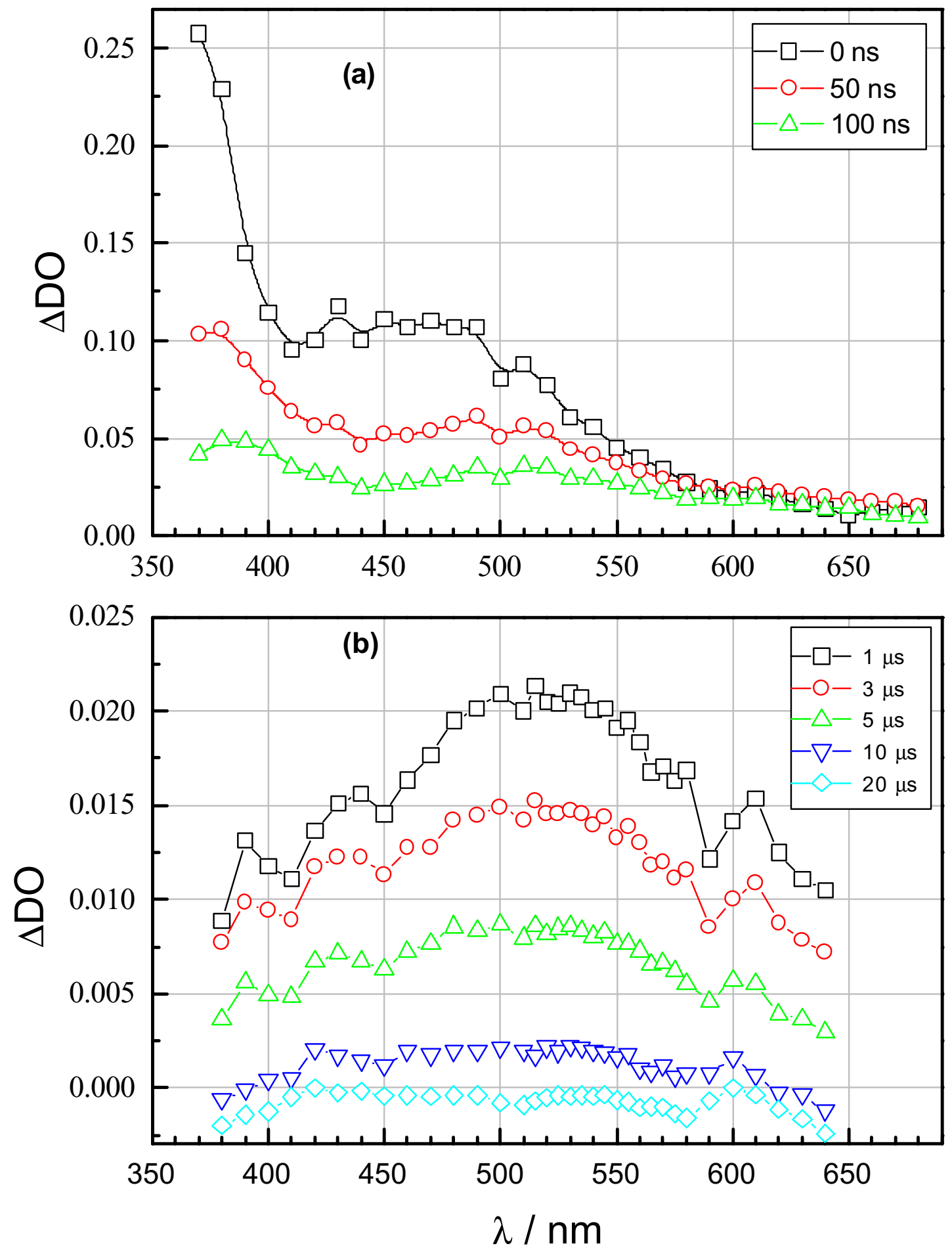


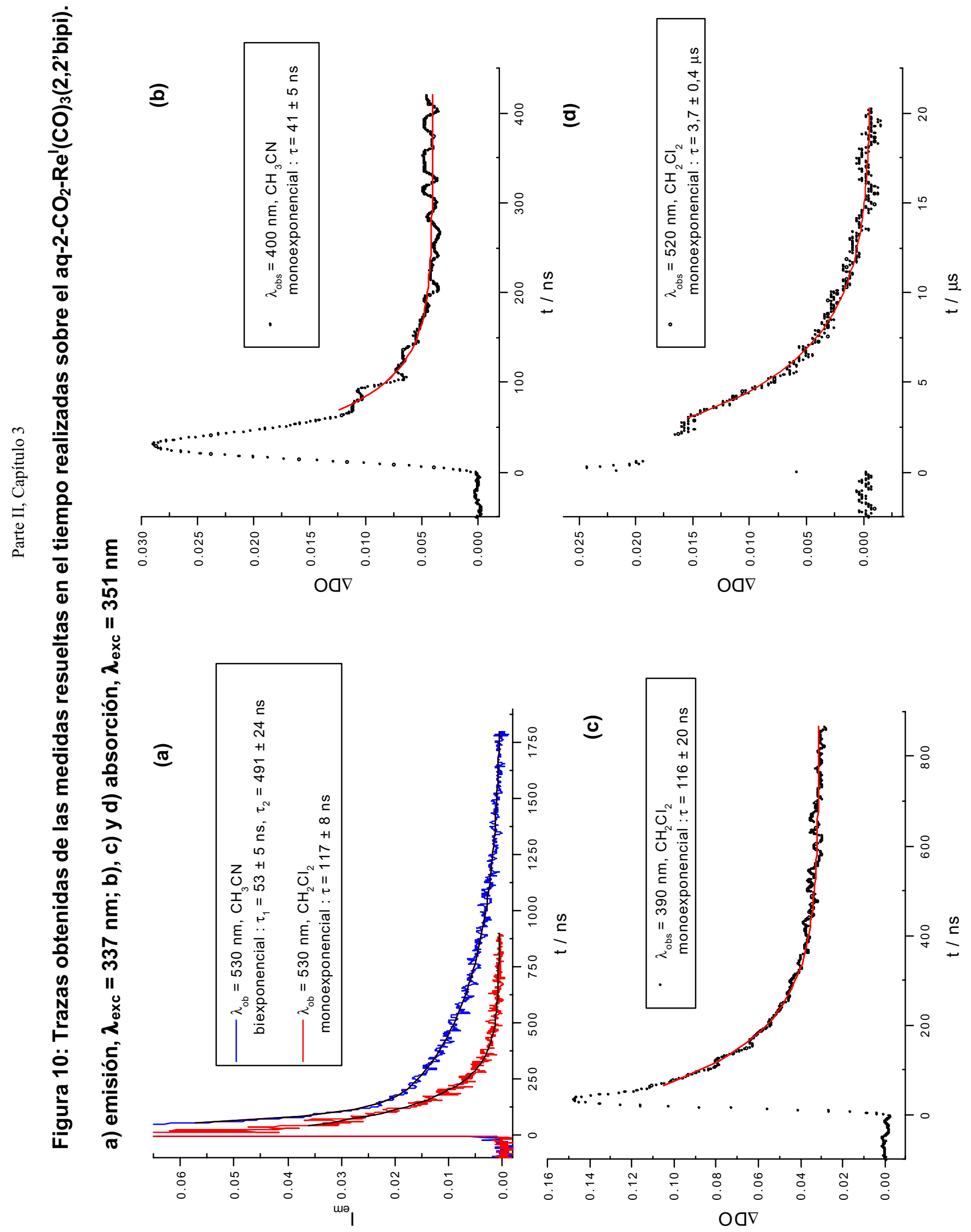


Además, la oxidación fotoinducida del diclorometano, causada por los estados excitados altamente reactivos, para producir el radical coordinado $2,2^{\prime}$ bipi $^{\circ-}$ fue investigada mediante la luminiscencia del complejo de $\mathrm{Re}^{\mathrm{I}}$ en estudio en $\mathrm{CH}_{2} \mathrm{Cl}_{2}$ y $\mathrm{CH}_{3} \mathrm{CN}$. Así, se pudo determinar que el rendimiento cuántico de emisión muestra un valor más grande en diclorometano que en acetonitrilo (ver tabla II) y que junto con los tiempos de vida de la luminiscencia en estos solventes, los datos sugieren una pequeña diferencia entre los valores de la constante de relajación radiativa y no radiativa del $\mathrm{TCML}_{\mathrm{Re} \rightarrow 2,2^{\prime} \text { bipi }}$ en ambos solventes. Sin embargo, la suma de estas dos constantes deben ser suficientemente grandes para superar la contribución proveniente de cualquier reacción del estado excitado con el solvente.

Tabla II: Vida media de emisión, $\tau_{\mathrm{emi}}$ y rendimiento cuántico de luminiscencia, $\phi$ emi como función del solvente y longitud de onda de excitación.

\begin{tabular}{|c|c|c|c|c|}
\hline Fotolito & ${ }^{\mathrm{a}} \tau_{\mathrm{emi}}, \mathrm{ns}$ & $\lambda_{\text {exc }}, \mathrm{nm}$ & Solvente & $\mathrm{b}_{\phi_{\mathrm{emi}}}$ \\
\hline \multirow[t]{4}{*}[\mathrm{aq}-2-\mathrm{CO}_{2}-\operatorname{Re}^{\mathrm{I}}(\mathrm{CO})_{3}(2,2^{\prime}\text{'bipi})]{} & $\begin{array}{l}(53 \pm 5) \\
(491 \pm 24)\end{array}$ & 350 & $\mathrm{CH}_{3} \mathrm{CN}$ & $(1,7 \pm 0,5) \cdot 10^{-4}$ \\
\hline & $(110 \pm 10)$ & & $\mathrm{CH}_{2} \mathrm{Cl}_{2}$ & $(1,06 \pm 0,05) \cdot 10^{-3}$ \\
\hline & & 400 & $\mathrm{CH}_{3} \mathrm{CN}$ & $(3,0 \pm 0,5) \cdot 10^{-4}$ \\
\hline & & & $\mathrm{CH}_{2} \mathrm{Cl}_{2}$ & $(2,6 \pm 0,1) \cdot 10^{-3}$ \\
\hline \multirow[t]{2}{*}{$\mathrm{aq}-2-\mathrm{CO}_{2} \mathrm{H}$} & 7500 & 350 & $\mathrm{CH}_{3} \mathrm{CN}$ & $(1,7 \pm 0,2) \cdot 10^{-3}$ \\
\hline & $\sim 10$ & & $\mathrm{CH}_{2} \mathrm{Cl}_{2}$ & $(8,6 \pm 0,5) \cdot 10^{-4}$ \\
\hline
\end{tabular}

a Medidos en un láser de $\mathrm{N}_{2}, \lambda_{\mathrm{exc}}=337 \mathrm{~nm}$ a temperatura ambiente

b Medidos a temperatura ambiente. 
La información experimental también sugiere una mínima o nula participación de los tripletes coordinados aq-2- $\mathrm{CO}_{2}$ y del $\mathrm{TCML}_{\mathrm{Re} \rightarrow \mathrm{aq}-2-\mathrm{CO} 2}$ en una oxidación eficiente del DCM por parte de estos estados excitados, ya que tendría que haberse observado en flash fotólisis el espectro del radical coordinado aq-2- $\mathrm{CO}_{2}{ }^{\bullet 2-}$.

Con el fin de obtener más información sobre la reactividad redox de los estados excitados, se llevaron a cabo experimentos de flash fotólisis a $351 \mathrm{~nm}$ utilizando trietilamina como donor de electrones, figura 11a. Se irradiaron soluciones deaereadas de aq-2- $\mathrm{CO}_{2}-\operatorname{Re}^{\mathrm{I}}(\mathrm{CO})_{3}\left(2,2^{\text {' bipi })}\right.$ $1.10^{-4} \mathrm{M}$ en DCM que contenían, además, TEA $1.10^{-3} \mathrm{M}$. El espectro asignado al estado excitado desaparece dentro del tiempo de escala del pulso del láser, es decir, 10 ns. Después de la irradiación pulsada, permanece una absorción cuyo espectro tiene $\lambda_{\max } \approx 520 \mathrm{~nm}$, el cual es asignado al producto de la reacción del complejo de $\mathrm{Re}^{\mathrm{I}}$ electrónicamente excitado con TEA. Cuando se compara el espectro de este producto con el de los radicales semiquinona de varias antraquinonas derivadas y con el espectro del radical aq-2- $\mathrm{CO}_{2}{ }^{\bullet 2-}$ se ve que está corrido hacia el rojo y no presenta estructura. En cambio, compara bien con el espectro del radical coordinado $2,2^{\prime}$ bipi $^{\bullet-}[11]$.

El conjunto de las observaciones experimentales sugieren que la oxidación de TEA por los

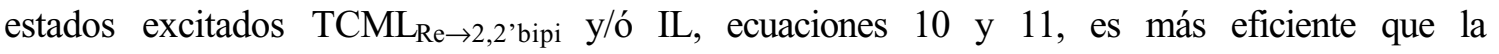
oxidación por tripletes del aq-2- $\mathrm{CO}_{2}$ coordinado, ecuación 12.

$$
\begin{aligned}
& \mathrm{IL}+\mathrm{TEA} \longrightarrow\left[\mathrm{aq}-2-\mathrm{CO}_{2}-\mathrm{Re}^{\mathrm{I}}(\mathrm{CO})_{3}\left(2,2^{\prime}{ }^{\prime} \mathrm{bipi}^{\circ}\right)\right]^{-}+\mathrm{TEA}^{\bullet+} \\
& \mathrm{TCML}_{\mathrm{Re} \rightarrow 2,2^{\prime}{ }^{\prime} \text { bipi }}+\mathrm{TEA} \longrightarrow\left[\mathrm{aq}-2-\mathrm{CO}_{2}-\mathrm{Re}^{\mathrm{I}}(\mathrm{CO})_{3}\left(2,2^{\prime}{ }^{\prime} \text { bipi }^{\bullet}\right)\right]^{-}+\mathrm{TEA}^{\bullet+}
\end{aligned}
$$


$\left\{\left[^{\mathrm{T}}\right.\right.$ aq-2- $\mathrm{CO}_{2}-\mathrm{Re}^{\mathrm{I}}(\mathrm{CO})_{3}\left(2,2^{\prime}\right.$ 'bipi $\left.)\right]+\left[{ }^{\mathrm{T}}\right.$ aq-2- $\mathrm{CO}_{2}{ }^{\prime}-\mathrm{Re}^{\mathrm{I}}(\mathrm{CO})_{3}\left(2,2^{\prime}\right.$ bipi $\left.\left.)\right]\right\}+\mathrm{TEA}$

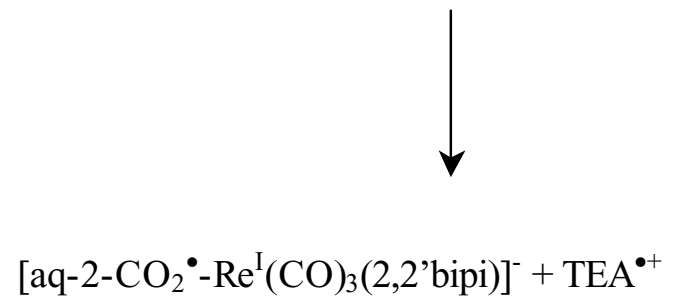

La disminuida reactividad redox de los tripletes aq-2- $\mathrm{CO}_{2}$ coordinados podría atribuirse al pequeño potencial redox de la cupla $\left({ }^{\mathrm{T}} \mathrm{aq}-2-\mathrm{CO}_{2}{ }^{-} \mathrm{y}^{\mathrm{T}} \mathrm{aq}-2-\mathrm{CO}_{2}{ }^{\prime-}\right) / \mathrm{aq}-2-\mathrm{CO}_{2}{ }^{\bullet 2-}$ comparado con los correspondientes a las cuplas $\operatorname{Re}^{\mathrm{II}} / \mathrm{Re}^{\mathrm{I}}$ e IL / 2,2'bipi- en los estados excitados respectivos, $\mathrm{TCML}_{\mathrm{Re} \rightarrow 2,2^{\prime} \text { bipi }}$ e IL. Algunos cambios espectrales observados a 500 ns luego del pulso de irradiación del láser, muestran la formación del radical aq-2- $\mathrm{CO}_{2}{ }^{\bullet 2-}$, figura $11 \mathrm{~b}$. Dadas las diferencias en los potenciales de media onda entre la cuplas 2,2'bipi / 2,2'bipi- y aq-2- $\mathrm{CO}_{2}^{-} /$aq-2- $\mathrm{CO}_{2}{ }^{-2-}$ los cambios espectrales podrían ser atribuidos a la relajación hacia el equilibrio entre los radicales coordinados $2,2^{\prime}$ bipi- $^{\bullet-}$ y aq-2- $\mathrm{CO}_{2}{ }^{\bullet 2-}$, ecuación $13 . \mathrm{El}$ decaimiento de estas especies, con un tiempo de vida $\tau=(3,5 \pm 0,4) \mu \mathrm{s}$, fue más lento que su llegada al equilibrio.

$\left[\mathrm{aq}-2-\mathrm{CO}_{2}-\mathrm{Re}^{\mathrm{I}}(\mathrm{CO})_{3}\left(2,2^{\prime} \mathrm{bipi}^{\bullet}\right)\right]^{-} \rightleftarrows\left[\mathrm{aq}-2-\mathrm{CO}_{2}{ }^{-}-\mathrm{Re}^{\mathrm{I}}(\mathrm{CO})_{3}\left(2,2^{\prime} \text { bipi }\right)\right]^{-}$ 
Figura 11: Cambios espectrales luego de irradiar, con pulsos de luz de $351 \mathrm{~nm}$, soluciones deaereadas de aq-2- $\mathrm{CO}_{2}-\operatorname{Re}^{\prime}(\mathrm{CO})_{3}\left(2,2^{\prime}\right.$ bipi $) 1,0.10^{-4}$ en $\mathrm{DCM}$ conteniendo $1,0.10^{-3} \mathrm{M}$ de TEA tomados con retardos de: a) 0 y $100 \mathrm{~ns}$; en el insertado: traza que muestra el crecimiento de la $D O$ a $\lambda_{\text {obs }}=530 \mathrm{~nm}$ asignado a la existencia del equilibrio entre $\left[\mathrm{aq}-2-\mathrm{CO}_{2}-\operatorname{Re}^{\prime}(\mathrm{CO})_{3}\left(2,2^{\prime} \mathrm{bipi}^{\circ}\right)\right]^{-}$y $\left[\mathrm{aq}-2-\mathrm{CO}_{2}{ }^{-}-\right.$ $\operatorname{Re}^{\prime}(\mathrm{CO})_{3}\left(2,2^{\prime}\right.$ 'bipi)] y b) $650 \mathrm{~ns}$ a $19 \mu \mathrm{s}$
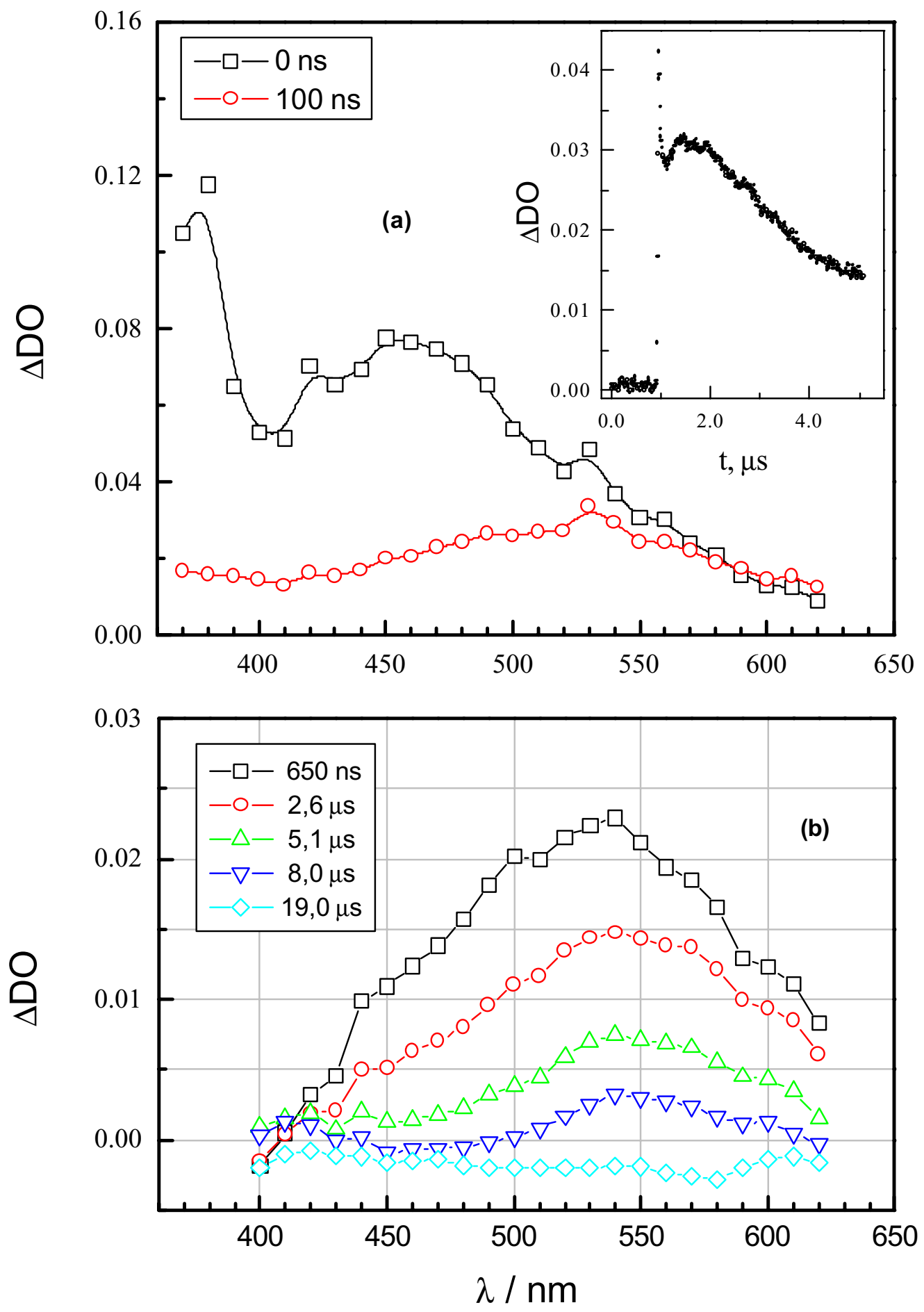


\section{Conclusiones}

El análisis de los resultados permite ahora relacionar las propiedades fotoquímicas $\mathrm{y}$ fotofísicas del aq-2- $\mathrm{CO}_{2}-\mathrm{Re}^{\mathrm{I}}(\mathrm{CO})_{3}(2,2$ 'bipi) con las propiedades de los estados excitados. Para ello, la construcción de un diagrama de Jablonski muestra las posiciones relativas de los estados excitados involucrados en la fotofísica y fotoquímica de este complejo de $\operatorname{Re}^{\mathrm{I}}$ cuando es excitado con enegías fotónicas $F_{h v}<29,6.10^{3} \mathrm{~cm}^{-1} \mathrm{~mol}^{-1}\left(\lambda_{\mathrm{exc}}=337 \mathrm{~nm}\right)$, figura 12a. Los potenciales redox, asignados en el complejo a las cuplas $\operatorname{Re}^{\mathrm{II}} / \mathrm{Re}^{\mathrm{I}}, 2,2^{\prime}$ bipi / 2,2'bipi- y aq-2$\mathrm{CO}_{2-}$ / aq-2- $\mathrm{CO}_{2-}{ }^{\bullet-}$, informados en la tabla $\mathrm{I}$, fueron usados para el cálculo de las energías de punto $0-0, \mathrm{E}_{00}$, de los estados excitados $\mathrm{TCML}_{\mathrm{Re} \rightarrow 2,2^{\prime} \text { bipi }}$ y $\mathrm{TCML}_{\mathrm{Re} \rightarrow \mathrm{aq}-2-\mathrm{CO}_{2}{ }^{7} \text {. De la }}$ luminiscencia de la 2,2'bipi protonada, se pudo asignar un valor de $\mathrm{F}_{0}$ IL de $\approx 23,5.10^{3} \mathrm{~cm}^{-1}$ $\mathrm{mol}^{-1}[15]$. Para los tripletes de la aq-2-CO $\mathrm{CO}_{2}$ se calcula un valor de $\mathrm{E}_{00} \approx 21,3 \cdot 10^{3} \mathrm{~cm}^{-1} \mathrm{~mol}^{-1} \mathrm{a}$ partir de los espectros de emisión que compara muy bien con los valores publicados [26-28]. Estos cálculos colocan a los estados excitados $\mathrm{IL}, \mathrm{TCML}_{\mathrm{Re} \rightarrow 2,2^{\prime} \text { bipi }} \mathrm{y}^{\mathrm{T}}$ aq-2- $\mathrm{CO}_{2}$ muy próximos en términos de energía, mientras que el $\mathrm{TCML}_{\mathrm{Re} \rightarrow \mathrm{aq}-2-\mathrm{CO}_{2}}$ se ubica a una energía más baja. Este diagrama de Jablonski sugiere entonces, que la pobre reactividad del $\mathrm{TCML}_{\mathrm{Re} \rightarrow \mathrm{aq}-2-\mathrm{CO}_{2}}$ hacia la oxidación de DCM ó TEA podría ser consecuencia de esta baja energía. Sin embargo, otros factores podrían afectar la reactividad de los estados excitados TCML. Por ejemplo, la aparente existencia de una distorsión significativa entre las configuraciones nucleares de

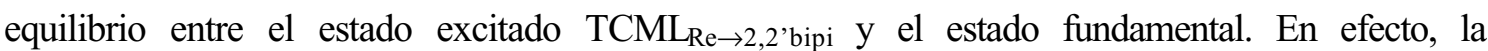

\footnotetext{
7) $\mathrm{E}_{00}$ es la diferencia de energía entre el estado fundamental y el estado excitado cuando ambos estados son tomados en sus niveles vibracionales cero y poseen el mismo spin. Para un estado excitado de transferencia de carga en un complejo del tipo donor / aceptor, (D/A), TCML $\mathrm{D}_{\mathrm{A}}$, la diferencia de potencial entre $\mathrm{D}^{+} / \mathrm{D}^{\mathrm{y}} \mathrm{A} / \mathrm{A}^{-}$ puede ser una buena aproximación para el valor relativo de energía del $\mathrm{TCML}_{\mathrm{D} \rightarrow \mathrm{A}}$ en su nivel vibracional cero. Por lo tanto, el valor de $\mathrm{E}_{00}(\mathrm{~V})=\mathrm{E}_{\mathrm{D}+/ \mathrm{D}}-\mathrm{E}_{\mathrm{A} / \mathrm{A} \text {-. }}+$ un término que contiene la energía de reorganización interna y la energía necesaria para pasar al estado triplete, $\Delta \mathrm{ST}$, que se calculan del espectro de emisión como se describe en el capítulo 1
} 
separación entre el máximo del espectro de absorción para la transición del $\mathrm{TCML}_{\mathrm{Re} \rightarrow 2,2^{2} \text { bipi, }} \mathrm{v}$ $\approx 25.10^{3} \mathrm{~cm}^{-1}$, y el valor de $\varpi_{0}$ en el espectro de emisión del aq-2- $\mathrm{CO}_{2}-\operatorname{Re}^{\mathrm{I}}(\mathrm{CO})_{3}\left(2,2^{2}\right.$ bipi $)$ corresponde a una energía de reorganización interna, $\lambda_{\text {org }} \approx 4000 \mathrm{~cm}^{-1}$. Este valor concuerda muy bien con el calculado con el promedio de las frecuencias IR de los modos vibracionales correspondientes al estiramiento $\mathrm{Re}^{\mathrm{I}}$-acina $\mathrm{y} \quad \mathrm{Re}^{\mathrm{II}}$-acina $\mathrm{y}$ la diferencia entre las correspondientes longitudes de enlace ${ }^{8)}[3,11,31,32]$. El efecto de la energía de reorganización y el desplazamiento de las superficies de energía potencial se muestran en la figura $12 \mathrm{~b},{ }^{9}$. Para el TCML $L_{\mathrm{Re} \rightarrow \mathrm{aq}-2-\mathrm{CO}_{2}}$ se podría esperar una energía de reorganización de similar magnitud. Sin embargo, la transición óptica para este estado excitado, debería esperarse en la región visible, es decir, a $\lambda \approx 550 \mathrm{~nm}$, sobre la base exclusiva de las energías calculadas para el $\mathrm{E}_{00} \mathrm{y}$ $\lambda_{\text {org. }}$ La ausencia de rasgos espectrales notables, atribuible a tal transición, en el espectro del aq-2- $\mathrm{CO}_{2}-\mathrm{Re}^{\mathrm{I}}(\mathrm{CO})_{3}\left(2,2^{\prime}\right.$ bipi $)$ y de transiciones similares en otros complejos de $\mathrm{Re}^{\mathrm{I}}$ con ligandos espectadores de carboxilatos relacionados [11], sugiere una baja probabilidad de transición. Es posible que una transferencia de carga no-adiabática fotoinducida entre el donor $\operatorname{Re}^{\mathrm{I}}$ y la antraquinona aceptora sea responsable de la baja probabilidad de transición y que la no-adiabaticidad de la transferencia resulte del desacoplamiento de las funciones de onda del donor y el aceptor inducido por el grupo carboxilato. Razones similares justifican también que

\footnotetext{
${ }^{8)}$ La energía de reorganización de enlace fue calculada con $\lambda_{\text {org }} \approx 3 / 2 \times \mathrm{fx}(\Delta \mathrm{d})^{2}$ [25] donde $\mathrm{f}=$ constante de fuerza media de enlace y $\Delta \mathrm{d}=$ diferencia en la distancia metal-ligando en el complejo oxidado y reducido.

${ }^{9)}$ Las curvas de energía potencial se calcularon bajo la aproximación armónica $\mathrm{E}=(1 / 2) \mathrm{k}\left(\mathrm{q}-\mathrm{q}_{0}\right)^{2}$ donde $\mathrm{k}$ es la constante de fuerza para el enlace $\mathrm{Re}^{\mathrm{I}}-\mathrm{L}$ y $\mathrm{Re}^{\mathrm{II}}-\mathrm{L}$, q es el desplazamiento, dado en cm, con respecto a la posición de equilibrio y $\mathrm{q}_{0}$ es la distancia de enlace en la posición de equilibrio para $\mathrm{Re}^{\mathrm{I}}-\mathrm{L}$ y $\mathrm{Re}^{\mathrm{II}}-\mathrm{L}$ tomado de datos espectroscópicos [31,32]. A las curvas correspondientes a los estados excitados se le adicionó el término $\mathrm{E}_{00}$ correspondiente.

Así, las tres curvas poseen las siguientes expresiones:

$\mathrm{E}_{\mathrm{estado}}$ fundamental $=3,2 \cdot 10^{29} \mathrm{erg} \cdot \mathrm{cm}^{-2}\left(\mathrm{q}-2 \cdot 10^{-8} \mathrm{~cm}\right)^{2}$

$\mathrm{E}_{\mathrm{Re} \rightarrow 2,2^{\prime} \mathrm{bipi}}=2,7 \cdot 10^{12} \mathrm{erg}+3 \cdot 10^{29} \mathrm{erg} \cdot \mathrm{cm}^{-2}\left(\mathrm{q}-1,85 \cdot 10^{-8} \mathrm{~cm}\right)^{2}$

$\mathrm{E}_{\mathrm{Re} \rightarrow \mathrm{aq}-2-\mathrm{CO} 2}=2,2 \cdot 10^{12} \mathrm{erg}+3 \cdot 10^{29} \mathrm{erg} \cdot \mathrm{cm}^{-2}\left(\mathrm{q}-1,85 \cdot 10^{-8} \mathrm{~cm}\right)^{2}$
} 
estados excitados IL, $\mathrm{TCML}_{\mathrm{Re} \rightarrow 2,2^{\prime} \text { bipi }} \mathrm{y}^{\mathrm{T}}$ aq-2- $\mathrm{CO}_{2}$ más altos no puedan poblar el estado excitado $\mathrm{TCML}_{\mathrm{Re} \rightarrow \mathrm{aq}-2-\mathrm{CO}_{2}}$ ya que si existieran conversiones eficientes de estados excitados de

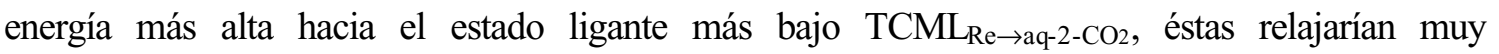
rápidamente al estado fundamental como se ha observado previamente en complejos de $\operatorname{Re}^{\mathrm{I}}$ con estados excitados TCML de baja energía [5]. Desde este punto de vista, entonces, el aislamiento del $\mathrm{TCML}_{\mathrm{Re} \rightarrow \mathrm{aq}-2-\mathrm{CO}_{2}}$ inducido por el carboxilato, provee un eficiente camino de reacción para la reducción indirecta del ligando espectador y que evita la total relajación al estado fundamental, ecuaciones 10 a 13. Este paso de reacción indirecta provee una manera de aprovechar la química redox del ligando espectador reducido 
Figura 12: a) Diagrama de Jablonski que muestra las energías de punto cero $\mathrm{E}_{00}$ de los niveles electrónicos relevantes para la fotólisis de aq-2- $\mathrm{CO}_{2-}$ $\operatorname{Re}^{\prime}(\mathrm{CO})_{3}\left(2,2^{\prime}\right.$ bipi). b) Curvas de energía potencial, calculadas con la aproximación armónica, que muestran el desplazamiento entre la del estado fundamental (-) y las de los $\mathrm{TCML}_{\mathrm{Re} \rightarrow 2,2^{\prime} \text { bipi }}(--)$ y $\mathrm{TCML}_{\mathrm{Re} \rightarrow \text { aq-2-CO2 }}($...)
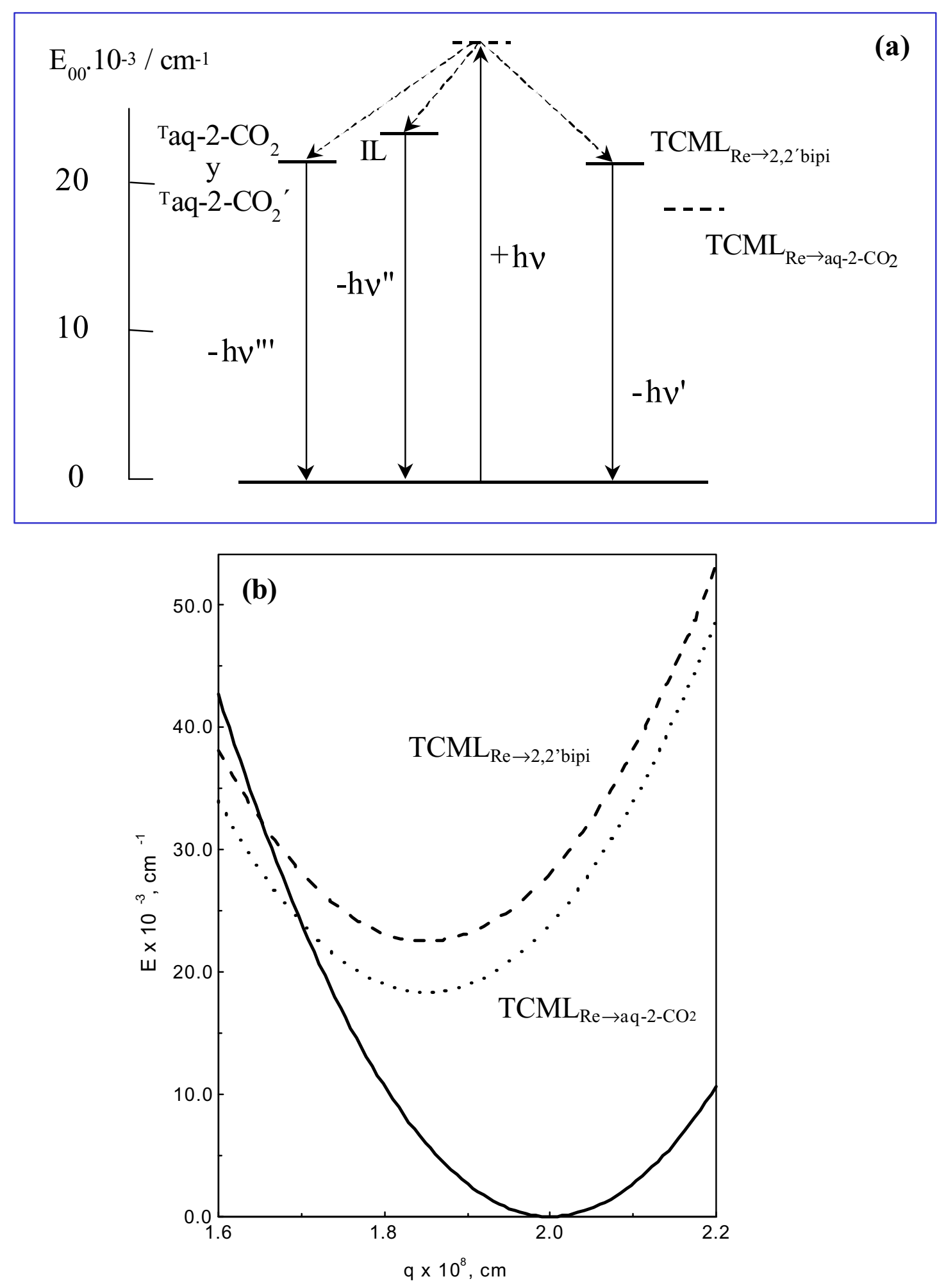
CAPITULO 4

\section{ESPECTROELECTROQUÍMICA UV-visible / IR DE fac-[Antraquinona-2-COO-Re $e^{I}(\mathrm{CO})_{3}(2,2$ 'bipiridina $\left.)\right]$}




\section{Resultados experimentales y Discusión}

\section{Materiales}

El complejo de $\mathrm{Re}^{\mathrm{I}}$, su precursor y los ligandos utilizados, se obtuvieron como se describe en el capítulo 2. También el espejado de los discos de Pt utilizados como electrodo de trabajo fue previamente descripto. El procedimiento de secado del acetonitrilo y del electrolito soporte, $\mathrm{Bu}_{4} \mathrm{NPF}_{6}$, permitió trabajar en un rango de potencial entre $-2.5 \mathrm{y}+0.6 \mathrm{~V}$ vs $\mathrm{Fc} / \mathrm{Fc}^{+}$. Una concentración típica de las soluciones de aq-2- $\mathrm{CO}_{2}-\mathrm{Re}(\mathrm{CO})_{3}(2,2$ 'bipi) utilizadas para los experimentos fue de $5.10^{-4} \mathrm{M}$ en $\mathrm{ACN}$.

\section{Equipos y métodos}

Los espectros UV-visible a potencial fijo fueron obtenidos iluminando el electrodo con un haz de luz con un ángulo de incidencia de $45^{\circ}$ y usando un analizador óptico multicanal computarizado (EG \& G PAR OMA III) equipado con una matriz de diodo de Si enfriado [33]. El detector, del tipo no intensificado, consiste de 1024 canales. Este espectrómetro de barrido rápido, con 14-bit de resolución, fue empleado para obtener espectros integrales resultantes de la co-adición en la memoria de la computadora de un número variable de exposiciones (dependiendo de la relación señal / ruido), cada uno promediando 0,03 s en el chip del arreglo de diodo. Los órdenes de difracción superiores a uno fueron cortados utilizando filtros apropiados.

Los espectros, como una especie de descarga instantánea, fueron adquiridos durante el progreso de lentas curvas voltamétricas, $\sim 5 \mathrm{mVs}^{-1}$, y fueron calculados como $\left(\mathrm{R}-\mathrm{R}_{\mathrm{ref}}\right) / \mathrm{R}_{\mathrm{ref}}$ con los espectros referencia, $\mathrm{R}_{\text {ref, }}$ tomados a potenciales apropiados. 
La obtención de espectros a más alta sensibilidad se consiguió midiendo con modulación del potencial del electrodo a $11 \mathrm{~Hz}$ a potenciales anteriores y posteriores al pico de la onda redox de interés, aprovechando la característica de proceso reversible de ésta. Las demodulaciones fueron llevadas a cabo con la ayuda de un amplificador de detección sincrónico ("lock-in"). Los espectros infrarrojo fueron obtenidos en el rango entre $1800-2100 \mathrm{~cm}^{-1}$. El electrodo de Pt fue colocado en forma ajustada justo en frente de la ventana óptica de $\mathrm{CaF}_{2}$, la cual tiene forma de disco, de manera de crear una delgada capa de solución de algunos micrómetros entre ambos. La técnica consiste en el uso de un espectrómetro dispersivo de alto pasaje cuya óptica dirige la radiación al electrodo en un ángulo de $60^{\circ}$ con respecto a la ventana de $\mathrm{CaF}_{2}$. La intensidad IR reflejada, fue modulada a $11 \mathrm{~Hz}$, por aplicación de una onda cuadrada al electrodo entre los límites de potencial convenientemente elegidos para cubrir un proceso redox específico. En algunas ocasiones, algunos espectros IR in-situ de tipo convencional fueron obtenidos de sus soluciones, por modulación de la luz a $80 \mathrm{~Hz}$ (“chopper”) y la celda electroquímica en estado de circuito abierto. Los cambios en la intensidad reflejada, como señal de corriente alterna $\mathrm{R}$ (reflectancia), fueron medidas paralelas al plano de incidencia $\mathrm{y}$ adquiridas por un detector MCT (mercurio-cadmio-teluro) de alta sensibilidad y de banda angosta. La salida del detector fue luego rectificada por un detector sincrónico, siendo el espectro final la diferencia normalizada entre la respuesta óptica a un potencial dado y la correspondiente al potencial tomado como referencia, $R_{\text {ref, }}$ es decir, $\Delta R / R_{\text {ref }}=\left(R-R_{\text {ref }}\right) / R_{\text {ref. }}$ La técnica ha sido descripta en detalle anteriormente (ver capítulo 1, [36,37]). Las voltametrías dc y ac fueron realizadas en la manera convencional y están descriptas en los capítulo 1 y 3 . 


\section{Medidas electroquímicas}

\section{i. $\quad$ Voltametría cíclica de alterna}

Como se describió anteriormente, los experimentos de voltametría ac realizados sobre soluciones de aq-2-CO $\mathrm{CO}_{2}-\mathrm{Re}^{\mathrm{I}}(\mathrm{CO})_{3}(2,2$ ’bipi), en un sistema electroquímico que lo podemos describir como $\mathrm{Pt}+\mathrm{ACN}+\mathrm{Bu}_{4} \mathrm{NPF}_{6}$ 0,1 M, mostró tres ondas de reducción reversibles a -1,24, $-1,67$ y $-1,77 \mathrm{~V}$ referidos a la cupla $\mathrm{Fc} / \mathrm{Fc}^{+}$, figura 1a (en todas las medidas electroquímicas se utilizó como electrodo de referencia $\left.\mathrm{Ag} / \mathrm{AgCl} / \mathrm{KCl}_{\text {sat }}\right)$. Comparando estos voltagramas con los del complejo precursor $\operatorname{BrRe}^{\mathrm{I}}(\mathrm{CO})_{3}\left(2,2^{\prime}\right.$ bipi $)$ y del ligando aq-2- $\mathrm{CO}_{2} \mathrm{H}$ medidos separadamente, se puede inferir que las reducciones a -1,24 y -1,67 V tienen lugar sobre el ligando antraquinona mientras que el proceso a $-1,77 \mathrm{~V}$ ocurre sobre la molécula de bipiridina. También se observó una onda anódica irreversible a +0,97 V asignada a la cupla $\operatorname{Re}^{\mathrm{I}} / \operatorname{Re}^{\mathrm{II}}$.

Las especies consideradas en este estudio fueron principalmente en solución; sin embargo no se puede descartar cierta cantidad preadsorbida a potenciales negativos previos al comienzo de los procesos de reducción. 
Figura 1: Voltagrama de corriente alterna en barrido catódico de una solución $5.10^{-4} \mathrm{M}$ del aq-2- $\mathrm{CO}_{2}-\mathrm{Re}^{\prime}(\mathrm{CO})_{3}\left(2,2^{\prime}\right.$ bipi $)$ en interfase con $\mathrm{Pt} / \mathrm{ACN} / 0,1 \mathrm{M}$ $\mathrm{Bu}_{4} \mathrm{NPF}_{6}$. Frecuencia $6 \mathrm{~Hz}$, amplitud 1,6 mV, v = 3,0 $\mathrm{mV} \mathrm{s}^{-1}$. Area de electrodo: $0,28 \mathrm{~cm}^{2}$.

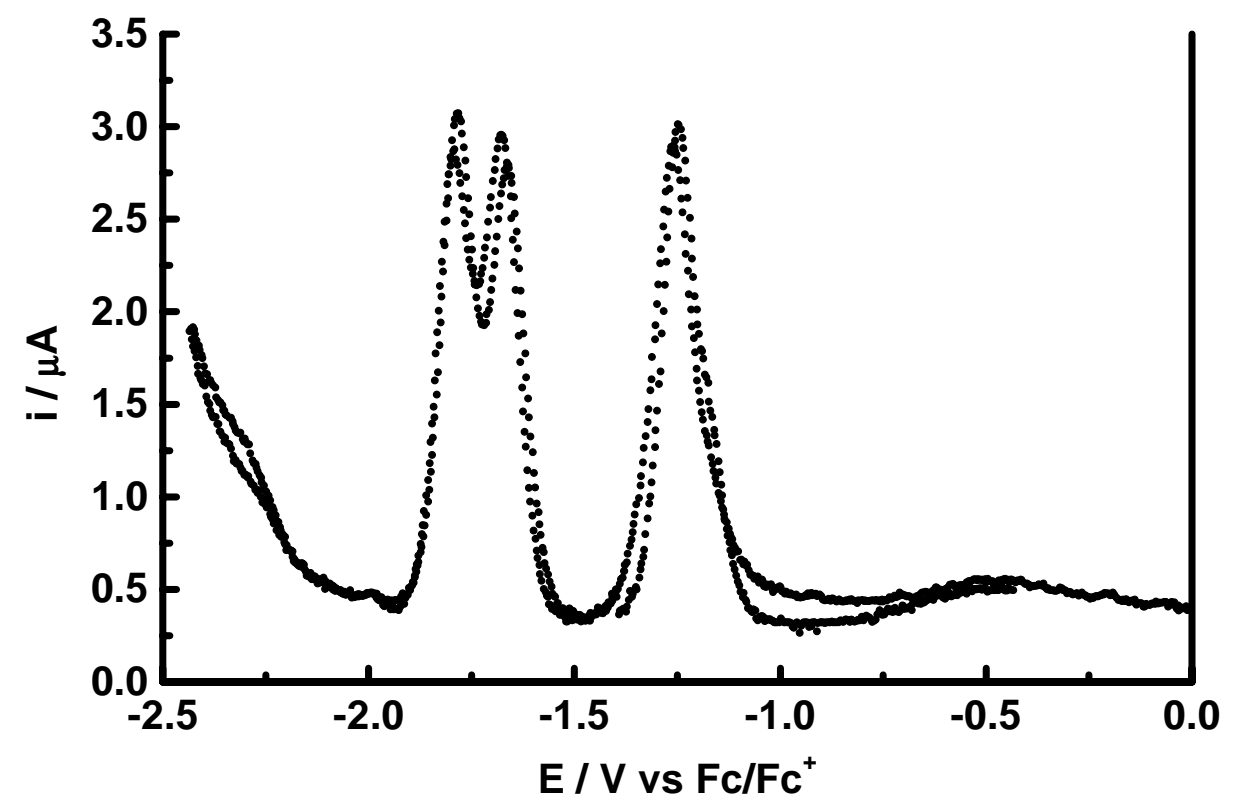

\section{ii. $\quad$ Espectros $U V$-visible de las especies reducidas}

Con el objetivo de identificar las especies generadas electroquímicamente, asociadas con las ondas obtenidas en la voltametría, se registraron in-situ los espectros UV-visibles del aq-2$\mathrm{CO}_{2}-\mathrm{Re}^{\mathrm{I}}(\mathrm{CO})_{3}\left(2,2^{\prime}\right.$ bipi) a intervalos de $50 \mathrm{mV}$ con la ayuda de un sistema OMA, mientras se realizaba un lento barrido negativo a $5 \mathrm{mVs}^{-1}$, entre $-0,43$ y $-2,4 \mathrm{~V}$. El espectro de referencia elegido fue el obtenido a $-0,43 \mathrm{~V}$ debido a que a este potencial no se detectaba ninguna absorción. La figura 2 exhibe, para clarificar, algunos de los espectros de las especies reducidas. Las bandas observadas para el potencial $-1,28 \mathrm{~V}$, a 583 y $420 \mathrm{~nm}$, provienen del 
producto de la primera reducción del ligando aq-2- $\mathrm{CO}_{2-}$, el anión radical aq-2- $\mathrm{CO}_{2^{-}}{ }^{--}$. Es de mucho valor hacer notar que este espectro es igual al obtenido cuando se reduce, mediante un donor de electrones como la trietilamina, al estado excitado triplete del aq-2- $\mathrm{CO}_{2} \mathrm{H}$ generado por excitación fotoquímica, como se describió en el capítulo 3, figura 6 [34].

A medida que el potencial se hace más negativo, una nueva banda a $494 \mathrm{~nm}$ aparece, aproximadamente a $-1,63 \mathrm{~V}$, indicando que ocurre una posterior reducción del aq-2- $\mathrm{CO}_{2^{-}}{ }^{-{ }^{-}}$, probablemente para dar el aq-2- $\mathrm{CO}_{2}{ }^{2-}$, que es el dianión que se obtiene como producto reducido final [34].

En el complejo de $\mathrm{Re}^{\mathrm{I}}$ en estudio, se encontró que la reducción del ligando 2,2’bipi, ocurre a 1,77 V para dar 2,2’bipi ${ }^{\bullet-}$ aunque las débiles bandas de absorción correspondientes se vieron enmascaradas e incluidas dentro de las bandas correspondientes a señales más intensas debidas a la reducción del aq-2- $\mathrm{CO}_{2^{-}}{ }^{--}$. Las absorciones, no claramente definidas en la figura 2, fueron obtenidas sin interferencias en medidas de alta sensibilidad realizadas sobre el $\operatorname{BrRe}^{\mathrm{I}}(\mathrm{CO})_{3}(2,2$ ’bipi $)$, utilizando modulación del potencial como se mostró en la figura 3, capítulo 3. Las bandas detectadas a 526, 496 y $366 \mathrm{~nm}$ en este espectro, nos muestran un interesante, aunque esperado, resultado en función de lo descripto anteriormente, que es la enorme similitud entre el espectro del ligando $2,2^{\text {’ bipi }}{ }^{\bullet-}$ obtenido electroquímicamente y la absorción del radical $\operatorname{BrRe}^{\mathrm{I}}(\mathrm{CO})_{3}\left(2,2^{\prime} \text { bipi }\right)^{\bullet-}$ generado en experimentos de flash fotólisis por quenching reductivo del estado excitado de transferencia de carga metal a ligando,

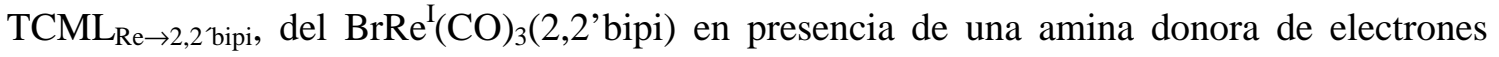
como la trietanolamina [23]. 


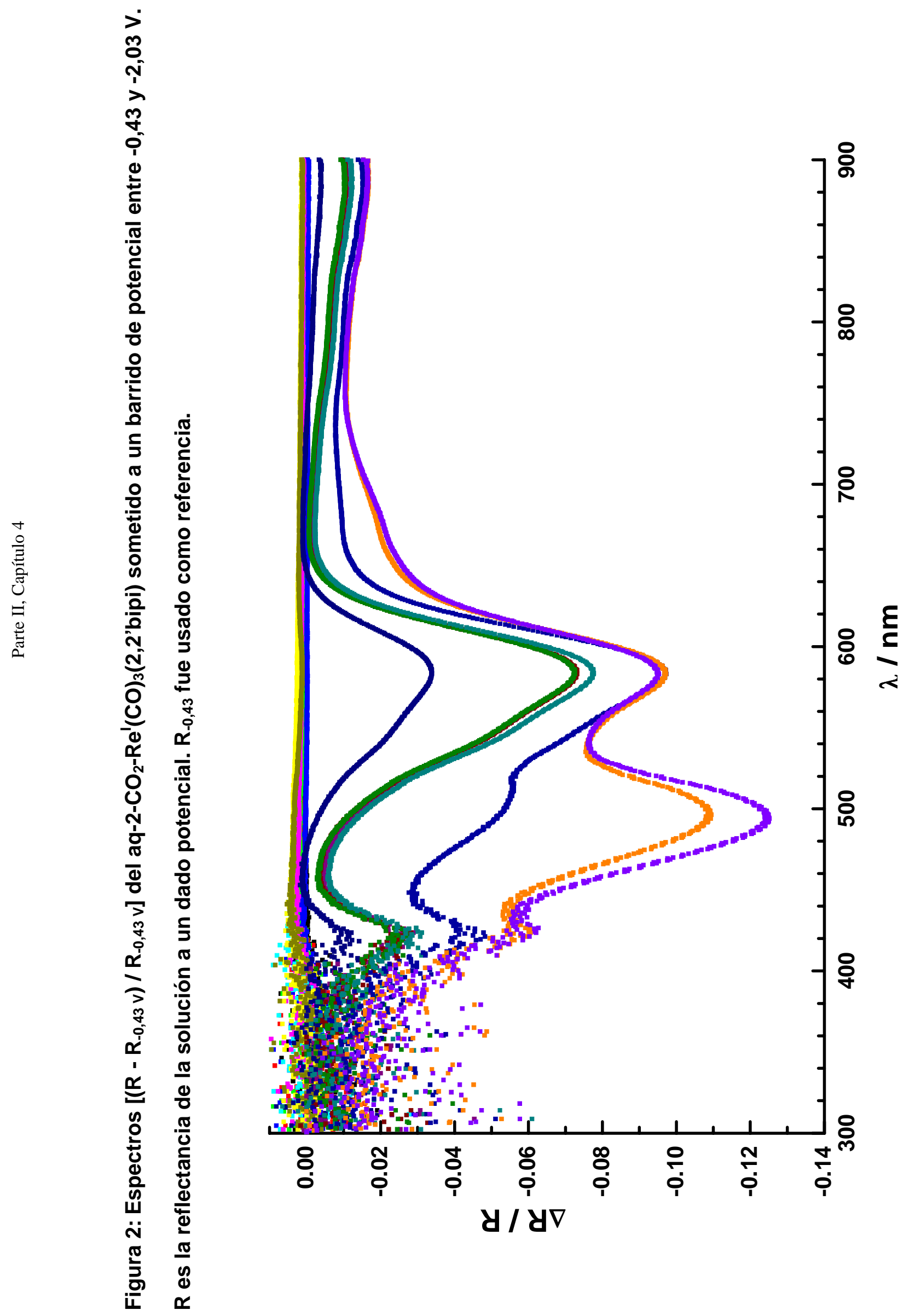




\section{iii. $\quad$ Espectros IR in-situ}

Los espectros IR in-situ fueron tomados en la región de los carbonilos, entre 1800 y $2100 \mathrm{~cm}^{-}$

${ }^{1}$, con la intención de explorar la redistribución de carga dentro del complejo en respuesta a las variaciones del potencial aplicadas sobre los diferentes ligandos del mismo, mediante los procesos redox generados por la inyección de carga. Lo que se espera es que los efectos de estos reacomodamientos en la distribución de la carga se vean reflejados en modificaciones en la frecuencia de estiramiento $v(\mathrm{C} \equiv \mathrm{O})$.

En la figura 3 se muestra el espectro de una solución del aq-2- $\mathrm{CO}_{2}-\mathrm{Re}(\mathrm{CO})_{3}(2,2$ ’ bipi), obtenido por modulación de la intensidad de luz ("chopper") a circuito abierto. Tres bandas características en la región de estiramiento $\mathrm{v}(\mathrm{C} \equiv \mathrm{O})$ son claramente observadas a 1896, 1916 y $2025 \mathrm{~cm}^{-1}$. 
Figura 3: Espectro IR in-situ del aq-2- $\mathrm{CO}_{2}-\operatorname{Re}^{\prime}(\mathrm{CO})_{3}\left(2,2^{\prime}\right.$ bipi), $\mathrm{R}$, tomado a circuito abierto. El espectro referencia, $\mathbf{R}_{0}$, corresponde al sistema sin complejo. Para la deconvolución se usaron perfiles de Lorentz. Area de electrodo: $0,95 \mathrm{~cm}^{2}$.

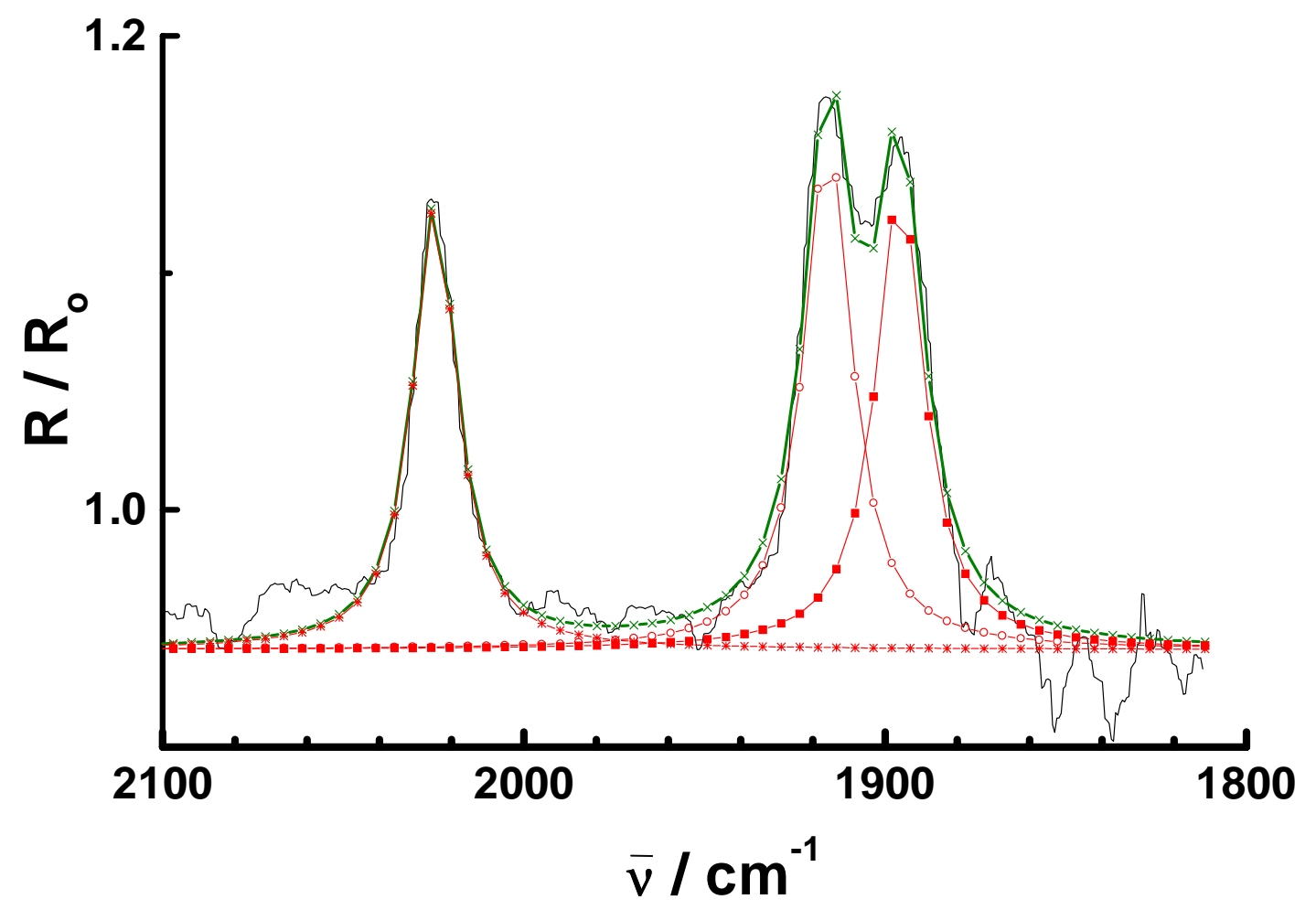

El efecto en el estiramiento de los carbonilos fue analizado después de la reducción de los ligandos 2,2'bipi ó aq-2- $\mathrm{CO}_{2^{-}}$y, en un experimento separado, de la oxidación del metal central Re ${ }^{\mathrm{I}}$. Para este fin, las medidas se llevaron a cabo independientemente sobre los complejos parientes aq-2- $\mathrm{CO}_{2}-\mathrm{Re}^{\mathrm{I}}(\mathrm{CO})_{3}\left(2,2^{\prime}\right.$ bipi $), \quad \mathrm{BrRe}^{\mathrm{I}}(\mathrm{CO})_{3}\left(2,2^{\prime}\right.$ bipi $)$ y $\mathrm{CH}_{3}-\mathrm{CO}_{2^{-}}$ $\operatorname{Re}^{\mathrm{I}}(\mathrm{CO})_{3}\left(2,2^{\prime}\right.$ bipi) (en lo que sigue $\mathrm{CH}_{3}-\mathrm{CO}_{2^{-}} \equiv \mathrm{Ac}$ ).

La modulación de potencial, es decir, las alteraciones de la carga debido a las reducciones, sobre las ondas redox reversibles de los ligandos 2,2'bipi y aq-2- $\mathrm{CO}_{2}$ - en el complejo de $\mathrm{Re}^{\mathrm{I}}$ 
en estudio y de la 2,2'bipi en los complejos relacionados con $\mathrm{Br}$ y acetato, mostraron modificaciones medibles y específicas en la frecuencia e intensidad de las bandas del carbonilo, figura 4. Por el contrario, la oxidación de $\operatorname{Re}^{\mathrm{I}}$ a $\operatorname{Re}^{\mathrm{II}}$ por el mismo método, no produce cambio alguno observable sobre las mismas bandas. Un detalle a tener en cuenta es que la oxidación de $\operatorname{Re}^{\mathrm{I}}$ fue irreversible.

Las bandas de los carbonilos luego de la modulación del potencial sobre la onda de reducción de los ligandos en el complejo con antraquinona fueron notablemente menos intensas que las obtenidas cuando se realiza la misma operación sobre la reducción de la 2,2’bipi en los complejos $\operatorname{BrRe}^{\mathrm{I}}(\mathrm{CO})_{3}\left(2,2^{\prime}\right.$ bipi $)$ ó $\mathrm{Ac}-\mathrm{Re}^{\mathrm{I}}(\mathrm{CO})_{3}(2,2$ 'bipi $)$. Además, si bien la primera reducción del ligando aq-2- $\mathrm{CO}_{2^{-}}$coordinado en el complejo de $\mathrm{Re}^{\mathrm{I}}$ en estudio, produce corrimientos medibles en la frecuencia de vibración $v(\mathrm{C} \equiv \mathrm{O})$, las intensidades resultantes de las bandas fueron más pequeñas que las observadas al reducir el ligando 2,2’ bipi en el mismo complejo. En todos los casos la señal más fuerte se originaba en la modificación de la carga sobre este último ligando ya sea en el $\operatorname{BrRe}^{\mathrm{I}}(\mathrm{CO})_{3}\left(2,2^{\prime}\right.$ bipi $), \operatorname{Ac}^{-} \mathrm{Re}^{\mathrm{I}}(\mathrm{CO})_{3}\left(2,2^{\prime}\right.$ bipi $)$ ó aq-2$\mathrm{CO}_{2}-\mathrm{Re}^{\mathrm{I}}(\mathrm{CO})_{3}\left(2,2^{\prime}\right.$ bipi $)$. Así, el efecto sobre $\mathrm{v}(\mathrm{C} \equiv \mathrm{O})$ es más importante cuando se coloca un electrón en la 2,2’bipi, debido, posiblemente, a la mejor conexión (solapamiento) entre los orbitales $\pi^{*}$ del ligando y los $\mathrm{d} \pi$ del $\mathrm{Re}^{\mathrm{I}}$. Por lo tanto, este ligando fue elegido como una especie de test indicador del fenómeno de transferencia de carga.

En la figura 4 se muestra el espectro IR del $A c-\operatorname{Re}^{\mathrm{I}}(\mathrm{CO})_{3}\left(2,2^{\prime}\right.$ 'bipi $)$ obtenido al modular el potencial entre -1,43 y -2,03 V cubriendo la onda voltamétrica de la 2,2’bipi. La absorbancia del mismo sistema obtenida a circuito abierto fue superpuesta para comparación. En la misma figura, se muestra el espectro del aq-2- $\mathrm{CO}_{2}-\mathrm{Re}^{\mathrm{I}}(\mathrm{CO})_{3}\left(2,2^{\prime}\right.$ bipi) obtenido al modular en la región del potencial de reducción de la 2,2’bipi, es decir, entre -1,73 y -1,93 V y donde se observa el rasgo distintivo de esta señal cuya intensidad es relativamente más débil que la correspondiente al complejo con acetato. 


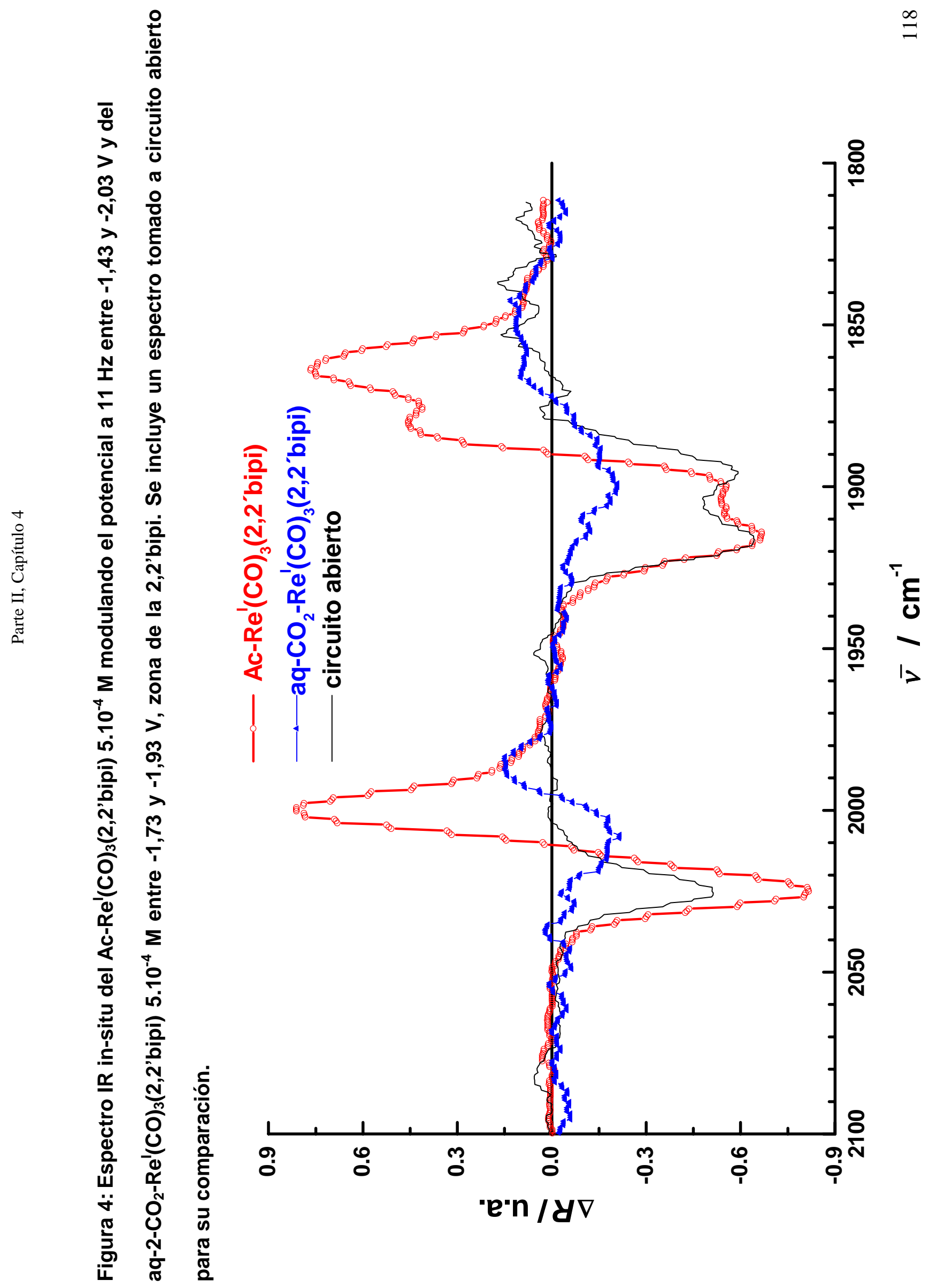


Cada una de las bandas del carbonilo, en la figura 4, se encuentran ahora divididas en dos lóbulos de signo opuesto mostrando un corrimiento en frecuencia como resultado de las variaciones de la carga sobre la molécula de 2,2’bipi. Los lóbulos positivos, que se observan a valores relativos de energía más bajos, denotan un incremento de la reflectancia de la radiación IR, es decir, una disminución de la absorción en la región final negativa de la modulación, -2,03 V en el caso del $A c-\mathrm{Re}^{\mathrm{I}}(\mathrm{CO})_{3}(2,2$ ’bipi). Por lo tanto, la inyección de carga en la molécula de 2,2'bipi se manifiesta en los ligandos carbonilos como un corrimiento de la frecuencia de estiramiento del enlace $\mathrm{C} \equiv \mathrm{O}$ hacia valores más bajos. Este debilitamiento del enlace $\mathrm{C} \equiv \mathrm{O}$ puede atribuirse a una retrodonación $\pi$ de los electrones $\mathrm{d} \pi$ del $\operatorname{Re}^{\mathrm{I}}$ metálico hacia los orbitales vacíos $2 \mathrm{p} \pi^{*}$ antienlazantes del $\mathrm{C} \equiv \mathrm{O}[35]$. La magnitud del corrimiento depende de la banda involucrada, observándose un corrimiento de $24 \mathrm{~cm}^{-1}$ para la absorción a frecuencias más altas y de 20 y $51 \mathrm{~cm}^{-1}$ para las bandas a menores energías.

El corrimiento observado en la frecuencia de vibración de los carbonilos, como consecuencia de la reducción de la 2,2'bipi, sugería la posibilidad de que, al oxidar al metal $\operatorname{Re}^{\mathrm{I}}$, se produjeran desplazamientos, posiblemente, de signo diferente a los observados en la reducción del ligando. Sin embargo, tales corrimientos no fueron detectados aún trabajando a altas sensibilidades. Este resultado podría indicar que la retrodonación solamente es significativa cuando el estado de oxidación formal del Re es igual a cero.

Los corrimientos en la frecuencia de los carbonilos fueron observados, no solamente para la reducción de la 2,2'bipi en el $A c-\operatorname{Re}^{\mathrm{I}}(\mathrm{CO})_{3}\left(2,2^{\prime}\right.$ bipi) sino también para la modulación del potencial en el complejo aq-2- $\mathrm{CO}_{2}-\operatorname{Re}^{\mathrm{I}}(\mathrm{CO})_{3}(2,2$ 'bipi), donde la inyección de carga fue aplicada selectivamente sobre las moléculas de aq-2- $\mathrm{CO}_{2}$ - ó de 2,2’bipi. Para ambos ligandos en el complejo en estudio, se encontró que las bandas del $\mathrm{C} \equiv \mathrm{O}$ resultantes eran de menor intensidad que las obtenidas con el complejo con acetato. La diferente simetría de ambos complejos podría explicar parcialmente las diferencias observadas. Sin embargo, como 
Parte II, Capítulo 4

venimos sosteniendo por los resultados presentados en el capítulo 3, las propiedades desacoplantes del grupo puente carboxilato para las funciones de onda del aceptor y del donor podría también estar relacionado con la pobre conductividad relativa entre la antraquinona y el Re metálico.

De todas maneras, la razón por la cual las bandas de IR de los carbonilos son menos intensas que en los complejos con Br ó acetato, no está aún muy clara. Podría deberse al hecho de que al potencial donde la 2,2'bipi se reduce, la molécula de antraquinona ya se encuentra en el estado reducido, por lo tanto, la perturbación inducida por la inyección de carga sobre la 2,2'bipi, en el complejo de $\operatorname{Re}^{\mathrm{I}}$ en estudio, podría ser proporcionalmente menos significativa que en los complejos con $\mathrm{Br}$ ó acetato (ambos sin antraquinona). Además, las posiciones de las bandas bipolares del carbonilo, fueron también diferentes al reducir a uno u otro ligando en el aq-2- $\mathrm{CO}_{2}-\mathrm{Re}^{\mathrm{I}}(\mathrm{CO})_{3}\left(2,2\right.$ 'bipi). La perturbación generada al reducir al aq-2- $\mathrm{CO}_{2}$ produjo desplazamientos de las bandas hacia frecuencias más altas (corrimientos más chicos) que aquellos producidos cuando la inyección de carga se realiza sobre la 2,2’bipi (la reducción de ésta se lleva a cabo a potenciales más negativos que la aq-2- $\mathrm{CO}_{2}$ ).

La aislación ejercida por el puente carboxilato podría también verse reflejada aquí, en cierta manera, "congelando" la carga sobre la molécula reducida aq-2- $\mathrm{CO}_{2}{ }^{--}$y por lo tanto, evitando una mayor retrodonación desde el Re metálico hacia el $\mathrm{C} \equiv \mathrm{O}$.

Los corrimientos en la frecuencia observados en las bandas de los carbonilos de estos complejos de $\mathrm{Re}^{\mathrm{I}}$, como resultado de la modificación indirecta de la carga sobre el metal central, son, en cierto modo, similares a los cambios, bien conocidos, que se producen en las vibraciones del estiramiento del enlace $\mathrm{C} \equiv \mathrm{O}$ de especies adsorbidas sobre electrodos de metales nobles en respuesta a las variaciones de carga en experimentos de electroquímica [37]. Diferencias menores observadas podrían deberse, probablemente, a desigualdades de 
simetría. Los electrodos metálicos en electroquímica y los complejos centrados en metales, $\mathrm{Re}$ en este estudio, han mostrado, entonces, que juegan un rol comparable en estos dos sistemas.

\section{Conclusiones}

La espectroelectroquímica in-situ UV-visible / IR del aq-2- $\mathrm{CO}_{2}-\mathrm{Re}^{\mathrm{I}}(\mathrm{CO})_{3}(2,2$ 'bipi) ha sido estudiada, habiéndose establecido que:

$3 / 4 \square$ Los espectros in-situ UV-visible de los aniones radicales reducidos electroquímicamente, aq-2- $\mathrm{CO}_{2}{ }^{\bullet-}$ y $2,2^{\prime}$ 'bipi- están en excelente concordancia con aquellos obtenidos de los productos del quenching reductivo correspondiente de los estados excitados poblados fotoquímicamente.

$3 / 4 \square$ Los corrimientos en la frecuencia de estiramiento de los ligandos $\mathrm{C} \equiv \mathrm{O}$ en respuesta a la inyección de carga sobre alguno de los ligandos del complejo, provee evidencia de la transferencia y reorganización de la carga dentro de la molécula. La oxidación del metal central $\mathrm{Re}^{\mathrm{I}}$ mostró que no produce cambios en el estiramiento del enlace $\mathrm{C} \equiv \mathrm{O}$.

$3 / 4 \square$ Se ha observado en el grupo aq-2- $\mathrm{CO}_{2}$ la característica de poseer una pobre conductividad electrónica adjudicada a las propiedades desacoplantes del grupo carboxilato. Este comportamiento es consistente también con la baja reactividad del estado excitado $\mathrm{TCML}_{\mathrm{Re} \rightarrow \mathrm{aq}-2-\mathrm{CO} 2}$. 
Bibliografía consultada para la Parte II del presente trabajo de tesis

[1] J. D. Lewis, R. N. Perutz, J. N. Moore, Chem. Commun. 2000, 1865.

[2] G. Knor, M. Leirer, A. Vogler, J. Organomet. Chem. 2000, 610, 19.

[3] J. Guerrero, S. A.Moya,M. T. Garland, R. F. Baggio, Acta Crystallogr., Sect. C 1999, 55, 932; S. A. Moya, J. Guerrero, R. Pastene, R. Schmidt, R. Sariego, R. Sartori, J. Sanzaparicio, I. Fonseca, M. Martinezripoll, Inorg. Chem. 1994, 33, 2341

[4] W. D. Bates, P. Y. Chem, W. E. Jones, T. J. Meyer, J. Phys. Chem. 1999, 103, 5227.

[5] G. Ferraudi, M. R. Feliz, E. Wolcan, I. Hsu, S. A. Moya, J. Guerrero, J. Phys. Chem. $1995,99,4929$.

[6] L. Sacksteder, A. P. Zipp, E. A. Brown, J. Streich, J. N. Demas, B. A. DeGraff, Inorg. Chem. 1990, 29, 4335.

[7] G. Ferraudi, M. R. Feliz, Inorg. Chem. 1998, 37, 2806.

[8] F. West, M. B. Scotian, P. Leibnitz, H. Spies, J. A. Katzenellenbogen, B. Johannsen, Bioorg. Med. Chem. 1999, 7, 1827.

[9] C. F. Shu and M.S. Wrighton, Inorg. Chem. 27 (1988) 4326.

[10] M.Féliz, G.Ferraudi, Inorg.Chem., 1998, 37, 2806.

[11] J.Guerrero, O.E.Piro, E. Wolcan, M.R.Féliz, G.Ferraudi, S.A.Moya, Organometallics $2001,20,2842$.

[12] M.S. Wrighton and D.L. Morse, J. Am. Chem. Soc., 100 (1978) 5790. P.J. Giordano, S.M. Fredericks, M.S. Wrighton and D.L. Morse, J. Am. Chem. Soc., 100 (1978) 2257. F. Zingales, U. Satorelli and A. Trovati, Inorg. Chem., 6 (1967) 1246. F. Zingales, M. Graziani, F. Faraone and U. Belluco, Inorg. Chim. Acta, 1 (1967) 172. Wender and P Pino (eds.), Organic Synthesis via Metal Carbonyls, Vol. 1, Interscience, New York, 1987. M.S. Wrighton, D.L. Morse and L. Pdungssap, J. Am. Chem. Soc., 97 (1975) 2073. 
[13] G. Ruiz, E. Wolcan, M.R. Féliz, J. Photochem. Photobiol. A: Chem. 101 (1996) 119125

[14] G. Ruiz, F. Rodriguez Nieto, E. Wolcan, M.R. Féliz, J. Photochem. Photobiol. A: Chem. 107 (1997) 47-54.

[15] G.Torchia, J.Tocho, O.E.Piro, P.Juliarena, G.Ruiz, E.Wolcan, M.R.Féliz. J. Chem. Soc, Dalton Trans., 2002 (10) 2194-2202.

[16] J.V. Caspar and T.J. Meyer, J. Phys. Chem. 1983, 87, 952-957.

[17] L. Sackteder, A.P. Zipp, E.A. Brown, J. Streich, J.N. Demas, B.A. DeGraff, Inorg. Chem., 1990, 29, 4335. E. Wolcan, G. Ferraudi, J. Phys. Chem. A 2000, 104, 9281.

[18] J.C. Luong, R.A. Faltynek and M.S. Wrighton, J. Am. Chem. Soc., 102 (1980) $7892-$ 7900.

[19] J.A. Barltrop and J.D. Coyle, "Principles of Photochemistry", Ed. John Wiley \& Sons, Bristol, 1978. Turro, N. "Modern Molecular Photochemistry", Ed. University Science Books, 1991.

[20] A.J. Bard and L.R. Faulkner, "Electrochemical Methods. Fundamentals and Applications”, Ed. John Willey \& Sons, New York, Inc. 1980

[21] G. Knor, M. Leirer, A. Vogler, J. Organomet. Chem. 2000, 610, 19.

[22] M.J. Snare, F.E Treloar, K.P. Ghiggino and P:J. Thistlethwaite, J. Photchem., 18,335346, 1982; T. Karstens and K. Kobs, J. Phys. Chem., 84, 1871-1872, 1980; F. Lopez Arbeloa, P. Ruiz Ojeda and I. Lopez Arbeloa, J. Liminesc., 44, 105-112, 1989.

[23] C. Kutal, M.A. Weber, G. Ferraudi, D. Geiger, Organometallics, 1985, 4, 2161.

[24] "Handbook Series in Organic Electrochemistry", Eds. L. Meites, P. Zuman, CRC Press, Cleveland, 1976, Vol. I, II.

[25] R. G. Wilkins, "Kinetics and Mechanism of Reactions of Transition Metal Complexes", 2nd edn., VCH Publishers, New York, 1991, pp. 263, 264. 
[26] I. Loeff, A. Treinin, H. Linschitz, J. Phys. Chem. 1983, 87, 2536.

[27] I. H. Leaver, in "Photochemistry of Dyed and Pigmented Materials", Eds. N. S. Allen and J. F. McKellar, Applied Science Publishers, Barking, Essex, England, 1980

[28] B. M. Krasovitskii, B. M. Bolotin, "Organic Luminescent Materials", VCH Publishers, New York, 1988.

[29] I. Loeff, J. Rabani, A. Treinin, H. Linschitz, J. Am. Chem. Soc. 1993, 115, 8933.

[30] B. E. Hulme, E. J. Land, G. O. Phillips, J. Chem. Soc., Faraday Trans. 1972, 68, 1992.

[31] K. Nakamoto, "Infrared and Raman Spectra of Inorganic and Coordination Compounds", $3^{\text {rd }}$ ed., Wiley-Interscience John Wiley \& Sons, New York, NY, 1981.

[32] R. D. Shannon, C. T. Prewitt, Acta Crystallogr., Sect. B 1969, 25, 925.

[33] R.O. Lezna, S. Juanto and J.H. Zagal, Electroanal. Chem. 389 (1995) 197.

[34] R. Frank, G. Greiner and H. Rau, Phys. Chem. Chem. Phys. 1 (1999) 3481.

[35] K. Nakamoto, "Infrared and Raman Spectra of Inorganic and Coordination Compounds", $4^{\text {th }}$ ed., John Wiley and Sons, New York, NY, 1986, p. 219.

[36] B. Beden, C. Lamy and J-M. Léger in "Modern Aspects of Electrochemistry" (Edited by J.M. Bockris, B.E. Conway and R. White), Vol. 22, p. 97. Plenum Press, New York (1992). R.O. Lezna, An. Asoc. Quím. Argent. 82(4), 293 (1994).

[37] R.O. Lezna, K. Kunimatsu, T. Ohtsuka and N. Sato, J. Electrochem. Soc. 134 (1987) 3090. 
PARTE III

SINTESIS, ESTUDIOS FOTOQUÍMICOS Y FOTOFÍSICOS DEL LIGANDO DPPZ (dipirido[3,2-a:2',3'-c]fenacina) Y DE UN COMPLEJO DE Re ${ }^{\mathrm{I}}$ DEL TIPO $[$ fac-(dppz $) \operatorname{Re}^{\mathrm{I}}(\mathrm{CO})_{3}\left(4,4^{\prime}\right.$ 'bipiridina $\left.)\right]\left(\mathrm{O}_{3} \mathrm{SCF}_{3}\right)$ 


\section{Introducción}

En los últimos años se han estudiado la fotoquímica y fotofísica de diferentes complejos de metales de transición que contenían ligandos basados en el dipirido[3,2-a:2'3'-c]fenacina (dppz), [1] habiéndose caracterizado los estados excitados que se generan luego de la absorción de luz. En particular, para complejos del tipo (dppz) $M(L){ }_{2}{ }^{+n}$ donde $M=\mathrm{Cu}^{\mathrm{I}}, \mathrm{Ru}^{\mathrm{II}}$, $\mathrm{Os}^{\mathrm{III}}, \mathrm{Rh}^{\mathrm{III}}, \mathrm{Co}^{\mathrm{III}}$, etc; y L = dppz, 1,10 fenantrolina, 2,2'bipiridina; se ha establecido que el estado excitado de más baja energía es un estado de transferencia de carga del metal al ligando, $\mathrm{TCML}_{\mathrm{M} \rightarrow \mathrm{L}}$. De manera similar, se han examinado las propiedades fotoquímicas y fotofísicas de una amplia gama de complejos de metales de transición d $\mathrm{d}^{6}$ del tipo fac(diimina) $\operatorname{Re}^{\mathrm{I}}(\mathrm{CO})_{3}(\mathrm{~L})^{+}$donde diimina se refiere a un ligando diimina bidentado como la 2,2’bipiridina y L un ligando monodentado como la piridina [2]. Esta familia de complejos presenta típicamente un estado excitado de más baja energía fuertemente luminiscente y de vida moderadamente larga que está basado en una transición del tipo d $\pi(\operatorname{Re}) \rightarrow \pi^{*}$ (diimina) [3].

Además, con respecto a las propiedades del estado excitado de esta familia de complejos de $\operatorname{Re}^{\mathrm{I}}$, se encuentran grandes similitudes con el prototipo para estados excitados TCML, el $\left(2,2^{\prime} \text { bipi }\right)_{3} \mathrm{Ru}^{+2}$, con excepción de pequeñas diferencias en la energía y velocidad de decaimiento no radiativo del estado excitado [3d]. A pesar de las fuertes similitudes en estos sistemas, una importante diferencia para los complejos de $\operatorname{Re}^{\mathrm{I}}$ es que contiene solamente un simple ligando diimina "cromofórico", es decir, no existe ambigüedad con respecto al ligando aceptor que está involucrado en la transición TCML [3d].

Sin embargo, para complejos de $\operatorname{Re}^{\mathrm{I}}$ del tipo $(\operatorname{dppz}) \operatorname{Re}^{\mathrm{I}}(\mathrm{CO})_{3}(\mathrm{~L})$, donde $\mathrm{L}=$ ligando piridínico, la asignación ha sido ambigua. Pocos trabajos se encuentran en la bibliografía para este tipo de complejos y postulan no solo la presencia del estado de TCML, sino también, la 
de un estado intraligante centrado en el dppz que sería el de menor energía [4-7]. Un estudio detallado sobre las propiedades fotoquímicas y fotofísicas del ligando tiene gran importancia para esclarecer esta asignación. Los trabajos encontrados en la bibliografía son muy pobres en este sentido y por ese motivo los estudios sobre tales propiedades del dppz tienen un lugar en este trabajo de tesis. Esos resultados serán comparados posteriormente con los obtenidos para un complejo de $\operatorname{Re}^{\mathrm{I}}$ con dppz.

Estos complejos, además, han despertado singular interés, por poseer la característica de interactuar con moléculas de ácido desoxiribonucleico, ADN, y presentar lo que se ha denominado como el efecto "ligth-switch". En efecto, esta característica de metalointercaladores con el ADN abre la posibilidad de desarrollar un nuevo campo de estudio con el objetivo de diseñar moléculas de complejos pequeñas que intercalen en secuencias específicas de ADN. Algunos ejemplos han generado gran expectativa en futuras aplicaciones como agentes quimioterapeúticos, como sondas selectivas para diferentes tipos de ADN y como agentes de diagnóstico altamente sensibles [8].

En los capítulos que siguen se describen la síntesis, caracterización y estudios fotofísicos y fotoquímicos del ligando dppz y de un complejo de $\operatorname{Re}^{\mathrm{I}}$ de este tipo, el [fac$(\mathrm{dppz}) \operatorname{Re}^{\mathrm{I}}(\mathrm{CO})_{3}\left(4,4^{\prime}\right.$ bipi $\left.)\right]\left(\mathrm{O}_{3} \mathrm{SCF}_{3}\right)^{1)}$. En un capítulo posterior se discuten los estados excitados involucrados y las propiedades redox de estos compuestos. Finalmente, estudios de "binding" con polinucleótidos sintéticos del tipo Poly[dAdT $]_{2}$ y Poly $[\mathrm{dCdG}]_{2}$ intentan hacer

\footnotetext{
1) Abreviaturas usadas en esta parte del presente trabajo de tesis: dipirido[3,2-a:2',3'-c]fenacina, dppz; 4,4'bipiridina, 4,4'bipi; ión triflato, $\mathrm{O}_{3} \mathrm{SCF}_{3}^{-} ; 1-10$ fenantrolina 5,6 diona, fendiona; especie transitoria, transiente; Trietanolamina, TEOA; Trietilamina, TEA; diclorometano, DCM; acetonitrilo, ACN; éter etílico, $\mathrm{Et}_{2} \mathrm{O}$; metanol, $\mathrm{MeOH}$; 2-propanol, 2-PrOH; tetrahidrofurano, THF; estado excitado de transferencia de carga Re a X, TCML $\mathrm{Re}_{\mathrm{X}}$; estado excitado intraligando, IL.
} 
un aporte concreto en la búsqueda y aplicación de complejos de $\operatorname{Re}^{\mathrm{I}}$ como sondas para el ADN dejando las puertas abiertas para futuras investigaciones en ese campo.

Los estructuras de los ligandos 4,4'bipiridina y dppz son mostrados a continuación

\section{4,4'bipiridina}

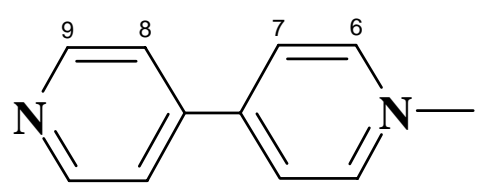

dppz

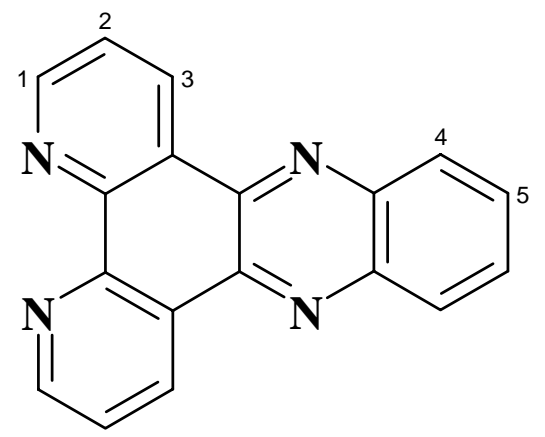




\section{CAPITULO 5}

\section{PREPARACION Y CARACTERIZACION DEL LIGANDO DPPZ (dipirido[3,2-a:2',3'-clfenacina) Y DEL $[$ fac-(dppz $) \operatorname{Re}^{\mathrm{I}}(\mathrm{CO})_{3}(4,4$ 'bipiridina $\left.)\right]\left(\mathrm{O}_{3} \mathrm{SCF}_{3}\right)$}




\section{Detalles Experimentales}

\section{Materiales}

Todos los solventes utilizados fueron de calidad proanálisis. Los reactivos 1-10 fenantrolina, 1,2 fenilendiamina, 4,4'bipiridina y $\mathrm{AgO}_{3} \mathrm{SCF}_{3}$, fueron adquiridos a Aldrich y se usaron sin posterior purificación. El precursor $\mathrm{Re}(\mathrm{CO})_{5} \mathrm{Cl}$ fue comprado a Strem Chemicals. El gel de sílica era de Riedel-de Haën. El bromuro de sodio se obtuvo de Mallinckrodt, el hidróxido de sodio de Merck y el sulfato de sodio anhidro de BDH Chemicals Ltd.

\section{Síntesis y caracterización de $\left[\right.$ fac-(dppz) $\mathrm{Re}^{\mathrm{I}}(\mathrm{CO})_{3}(4,4$ 'bipi $\left.)\right]\left(\mathrm{O}_{3} \mathrm{SCF}_{3}\right)$}

La obtención final del complejo de $\operatorname{Re}^{\mathrm{I}}$ en estudio involucra una serie de pasos y etapas entre las cuales se encuentra la de la síntesis del ligando dppz. Para obtenerlo, debemos sintetizar previamente su precursor, la 1-10 fenantrolina 5,6 diona, ya que no se encuentra disponible comercialmente. Esta es una síntesis en la cual la purificación del compuesto es muy complicada y que ha sido ampliamente discutida en la bibliografía con el fin de obtener mejores rendimientos [9-11]. El procedimiento descripto en esos trabajos ha sido modificado por nosotros, convenientemente, con el fin de asegurarnos la mayor pureza y rendimiento en nuestras condiciones de trabajo. En definitiva, los compuestos orgánicos y complejos precursores obtenidos fueron los siguientes:

\section{i. 1-10 fenantrolina 5,6 diona}


La obtención de este ligando orgánico se realiza, básicamente, a través de una fuerte oxidación de la 1-10 fenantrolina. Para ello se disuelven $3 \mathrm{~g}$ de fenantrolina en $20 \mathrm{ml}$ de ácido sulfúrico concentrado, en caliente, agitando lentamente. Se agregan 1,5 g de bromuro de sodio e inmediatamente, $10 \mathrm{ml}$ de ácido nítrico $70 \%$. Se calienta a reflujo durante $40 \mathrm{~min}$. Se deja enfriar y se vierte la mezcla sobre hielo, neutralizando con el agregado de hidróxido de sodio 10 M hasta alcanzar ph 7. Se deja decantar la solución durante 30 min. y se filtra. Luego, el precipitado se lava con agua hirviendo. Estas aguas de lavado se juntan con el filtrado y se procede a la extracción con diclorometano en ampolla de decantación. El extracto orgánico se seca sobre sulfato de sodio anhidro y se evapora a presión reducida. El residuo cristalino amarillo-naranja de fendiona finalmente se recristaliza desde tolueno o metanol. El punto de fusión y el espectro de absorción UV-visible de soluciones en DCM permitieron caracterizar el compuesto $[9,10]$.

\section{ii. $\quad$ fac-(1-10 fenantrolina 5,6 diona $) \operatorname{Re}^{\mathrm{I}}(\mathrm{CO})_{3} \mathrm{Cl}$}

Con la fendiona así obtenida se prepara una suspensión de 510 mg, 2,42 mmoles, en $20 \mathrm{ml}$ de tolueno junto con $721 \mathrm{mg}, 1,99$ mmoles, de $\operatorname{Re}(\mathrm{CO})_{5} \mathrm{Cl}$. Se calienta a reflujo durante $4 \mathrm{hs}$, manteniendo la agitación y la atmósfera de $\mathrm{N}_{2}$. Se enfría y el sólido resultante se colecta por filtración en vacío. El producto crudo se purifica por cromatografía en columna sobre gel de sílica usando tetrahidrofurano como eluyente. El eluato se evapora a presión reducida, se redisuelve en diclorometano y se reprecipita por el agregado de isooctano obteniéndose cristales marrones de $f a c$-(fendiona) $\operatorname{Re}^{\mathrm{I}}(\mathrm{CO})_{3} \mathrm{Cl}$ (Rto. $70 \%$ )

iii. $\quad$ fac-(dppz) $\operatorname{Re}^{I}(\mathrm{CO})_{3} \mathrm{Cl}$ 
Una solución en etanol absoluto de $f a c$-(fendiona) $\mathrm{Re}^{\mathrm{I}}(\mathrm{CO})_{3} \mathrm{Cl}(400 \mathrm{mg}$ en $20 \mathrm{ml}$ de EtOH$)$, se purgó con $\mathrm{N}_{2}$ adicionando posteriormente fracciones de 1,2 fenilendiamina (103 mg en $5 \mathrm{ml}$ de EtOH). En estas condiciones se produce la síntesis in-situ del dppz, es decir, se lo obtiene por una reacción de condensación con su precursor ya introducido en la esfera de coordinación del Re.

Esta solución se calienta a reflujo durante $1 \mathrm{~h}$. Transcurrido ese tiempo se deja enfriar, se filtra y seca en vacío. Se obtienen cristales de color naranja de $f a c-(\operatorname{dppz}) \operatorname{Re}^{\mathrm{I}}(\mathrm{CO})_{3} \mathrm{Cl}$. El producto se caracterizó por espectroscopía UV-visible determinando los coeficientes de extinción en diclorometano. Estos estuvieron en franca concordancia con los que se encuentran en la literatura [7] (Rto. $80 \%$ )

Los siguientes pasos de síntesis tienen como objetivo incorporar en la esfera de coordinación del $\operatorname{Re}^{\mathrm{I}}$ a la 4,4'bipiridina, por sustitución del cloruro, siguiendo una estrategia que se encuentra en la bibliografía [6,12], pero con pequeñas modificaciones que mejoran la eficiencia de la reacción.

iv. $\quad\left[\mathrm{fac}-(\mathrm{dppz}) \mathrm{Re}^{\mathrm{I}}(\mathrm{CO})_{3}\left(\mathrm{CH}_{3} \mathrm{CN}\right)\right]\left(\mathrm{O}_{3} \mathrm{SCF}_{3}\right)$

Se disuelven cantidades equimolares del complejo $f a c-(d p p z) \operatorname{Re}^{\mathrm{I}}(\mathrm{CO}){ }_{3} \mathrm{Cl}$ ( $330 \mathrm{mg}, 6.10^{-4}$ moles) y de $\mathrm{AgO}_{3} \mathrm{SCF}_{3}\left(157 \mathrm{mg}, 6.10^{-4}\right.$ moles $)$ en $200 \mathrm{ml}$ de acetonitrilo y se calienta a reflujo y en atmósfera de $\mathrm{N}_{2}$ durante $\sim 16$ hs. Se enfría, se deja reposar y se filtra el $\mathrm{AgCl}$ insoluble formado. A la solución concentrada de producto de síntesis, que queda como remanente retenida entre el sólido de $\mathrm{AgCl}$, se la somete a una extracción por Soxleth durante 2 días. El extracto se evapora a presión reducida y se recristaliza con DCM / isooctano obteniéndose cristales amarillos del complejo buscado. (Rto. > $90 \%$ ) 


\section{v. $\quad\left[f a c-(d p p z) \operatorname{Re}^{I}(C O)_{3}\left(4,4^{\prime}\right.\right.$ bipi $\left.)\right]\left(O_{3} S C F_{3}\right)$}

A una solución de $\left.(\mathrm{dppz}) \mathrm{Re}^{\mathrm{I}}(\mathrm{CO})_{3}\left(\mathrm{CH}_{3} \mathrm{CN}\right)\right]\left(\mathrm{O}_{3} \mathrm{SCF}_{3}\right)$ ( $380 \mathrm{mg}, 5.10^{-4}$ moles) en THF (200 $\mathrm{ml})$ se le adiciona una cantidad 15 veces mayor en moles de 4,4'bipiridina $\left(1,2 \mathrm{~g}, 8.10^{-3}\right.$ moles) y se mantiene durante 4 hs en las mismas condiciones de reflujo anteriormente descriptas. Se obtiene un precipitado amarillo-verde del complejo deseado agregando isooctano gota a gota. El sólido se recristalizó repetidas veces en DCM / isooctano hasta constancia de los coeficientes de extinción. Por cromatografía en capa delgada en gel de sílice, se verificó la eliminación gradual después de cada recistalización de la 4,4’bipiridina residual. Por último, se lava con $\mathrm{Et}_{2} \mathrm{O}$ y se seca en vacío (Rto. > $70 \%$ )

Análisis elemental. Calculado para $\mathrm{C}_{32} \mathrm{H}_{18} \mathrm{~N}_{6} \mathrm{O}_{6} \mathrm{ReF}_{3} \mathrm{~S}, \mathrm{M}=857,8 \mathrm{~g} / \mathrm{mol}: \mathrm{C}$ 44,80 \%, H 2,11 $\%$, N $9,80 \%$, S 3,74 \%. Encontrado: C 44,78 \%, H 2,11\%, N $9.13 \%$, S 3,78\% La síntesis del $d p p z$ se puede realizar también en forma separada por un procedimiento similar al descripto en el ítem iii., condensando la 1,2 fenilendiamina (20\% en exceso) con la fendiona en etanol a reflujo y en atmósfera de $\mathrm{N}_{2}$. El procedimiento es rápido, sencillo y de buen rendimiento $(\approx 50 \%)$. El sólido amarillo-anaranjado se recristaliza desde metanol [10]. Mejores rendimientos se obtuvieron calentando la mezcla en tolueno durante 2 horas y recristalizando desde EtOH. Luego, el complejo precursor de $\operatorname{Re}^{\mathrm{I}} f a c-(\mathrm{dppz}) \mathrm{Re}^{\mathrm{I}}(\mathrm{CO}){ }_{3} \mathrm{Cl}$ puede obtenerse por combinación directa entre el dppz y el $\operatorname{Re}(\mathrm{CO})_{5} \mathrm{Cl}$, como se ha descripto para la obtención de complejos similares [4,7]. Este complejo precursor se ha obtenido también por esta vía, en el presente trabajo de tesis, con resultados positivos. 


\section{Técnicas y equipamiento utilizados}

Las especificaciones de los equipos utilizados para la obtención de los espectros UV-visible y voltametría cíclica, ya fueron descriptos anteriormente (ver Parte I, capítulo 1 y Parte II, capítulo 2). En el caso en que las condiciones difieran, se aclarará convenientemente para cada caso en particular.

Los espectros IR con transformada de Fourier (FTIR) fueron tomados entre 550 y $4000 \mathrm{~cm}^{-1}$ usando soluciones concentradas del complejo de $\mathrm{Re}^{\mathrm{I}}$ en estudio en ACN para observar bien, fundamentalmente, las bandas de absorción de los carbonilos. El equipo utilizado fue un Bruker IFS 113v.

La resonancia magnética nuclear de hidrógeno, ${ }^{1} \mathrm{H}-\mathrm{RMN}$, se llevó a cabo en un equipo Bruker, AC 250, que corresponde a una frecuencia de $250 \mathrm{MHz}$, a $296 \mathrm{~K}$. Para preparar las soluciones se utilizó $\mathrm{ACN}$ deuterado, $\mathrm{CD}_{3} \mathrm{CN}$.

El análisis fotofísico detallado de los espectros de absorción UV-visible será realizado en el capítulo 6 y 7 . El objetivo, en este capítulo, es el de identificar las transiciones observadas en las bandas de absorción y utilizar las asignaciones correspondientes de manera similar a lo descripto en el capítulo 2. Aquí, simplemente, intentamos, entonces, encontrar en el espectro de absorción del complejo de $\mathrm{Re}^{\mathrm{I}}$ en estudio, transiciones previamente observadas en los espectros correspondientes a los ligandos libres, con el fin de caracterizar al complejo, interpretando que la presencia de esas bandas de absorción sugerirían que los ligandos estarían coordinados, formando parte integrante del complejo.

Las consideraciones tenidas en cuenta para el análisis del espectro IR también fueron establecidas en el capítulo 2 y, por lo tanto, los detalles de la interpretación se omitirán en este capítulo. 


\section{Resultados}

\section{Síntesis}

La ruta de síntesis utilizada para la obtención del complejo de $\operatorname{Re}^{\mathrm{I}}$ se ilustra en el esquema I. El primer paso consiste en la oxidación de la fenantrolina para dar fendiona. La reacción en sí es muy sencilla pero la formación de compuestos intermedios nitrados en las posiciones 5 y 6 de la fenantrolina complican la purificación posterior. Esta es la principal causa del bajo rendimiento de esta reacción. Los primeros trabajos sobre la síntesis de este compuesto obtenían sólo un $15 \%$ de rendimiento [10]. Posteriormente, el agregado de BrNa, mejoró las condiciones de oxidación, aunque los rendimientos finales seguían siendo bajos [9,13,14]. Trabajos posteriores exploraron nuevas vías de síntesis logrando mejorar notablemente los rendimientos llevándolos a valores del orden del $80 \%$ [11,15]. El procedimiento utilizado para la síntesis de la fendiona estuvo condicionado a los materiales y equipos disponibles en nuestro laboratorio y, aunque el procedimiento seguido involucraba rendimientos bajos, ajustando algunos detalles experimentales, se consiguió mejorar el rendimiento y, además, se obtuvo el producto con alto grado de pureza.

Una vez obtenido este precursor orgánico, la síntesis del dppz no presentaba mayores complicaciones [10,15]. Este pudo sintetizarse por condensación de la base de Schiff con el compuesto diamino apropiado, es decir la 1,2 fenilendiamina (de esta forma se obtienen también los diferentes dppz sustituidos utilizando la correspondiente diamina). Sucesivas recristalizaciones hasta constancia de los $\varepsilon$, permitieron comprobar por comparación con lo encontrado en la bibliografía, que el compuesto había sido obtenido libre de impurezas. La caracterización del ligando libre, se complementó con la obtención de los potenciales redox, 
espectroscopía IR y ${ }^{1} \mathrm{H}-\mathrm{RMN}$ (ver más adelante). El ligando así obtenido fue utilizado para las medidas fotoquímicas y fotofísicas presentadas en el capítulo 6.

A partir de aquí podíamos introducir el dppz en la esfera de coordinación del Re siguiendo el procedimiento clásico, ampliamente utilizado para obtención de complejos de $\operatorname{Re}^{\mathrm{I}}$ diiminos relacionados [12]. Con este procedimiento tuvimos resultados positivos, no obstante, decidimos intentarlo coordinando la fendiona primero, para producir el complejo de $\operatorname{Re}^{\mathrm{I}}$ correspondiente, y generar, en un paso posterior, el dppz in-situ. Ambas alternativas mostraron ser efectivas para la generación del precursor buscado [5].

La coordinación de una piridina sustituida en complejos de $\mathrm{Re}^{\mathrm{I}}$ con ligandos bidentados requiere la eliminación del ión $\mathrm{Cl}^{-}$. Para ello, generalmente, se hace precipitar $\mathrm{el}^{-}$con una sal de $\mathrm{Ag}^{+}$, como el $\mathrm{AgO}_{3} \mathrm{SCF}_{3}$, y en el mismo balón se adiciona solución de la piridina a introducir con un exceso mayor al 20 \% en moles para asegurar la sustitución. Así se ha hecho para complejos relacionados en donde la piridina a incluir fue piridina [4], 4 metilpiridina [5] ó 4 etilpiridina [15]. En otros trabajos se producía la precipitación del $\mathrm{AgCl}$ sustituyendo al $\mathrm{Cl}^{-}$por el ión lábil $\mathrm{O}_{3} \mathrm{SCF}_{3}^{-}$y en una etapa posterior se introducía la piridina elegida $[6,15]$. Si la eliminación del $\mathrm{Cl}^{-}$, siguiendo esa estrategia, se realiza en acetonitrilo, se obtiene un complejo que tiene en su esfera de coordinación, una molécula de $\mathrm{CH}_{3} \mathrm{CN}$. Este ligando es fácilmente reemplazado por piridinas en soluciones de solvente adecuado. Este último procedimiento permitió la inclusión de la 4,4’bipiridina en un tiempo de síntesis más corto, y el uso de menor cantidad de ligando en exceso. Adicionalmente, se obtuvo un alto rendimiento. 
$n$

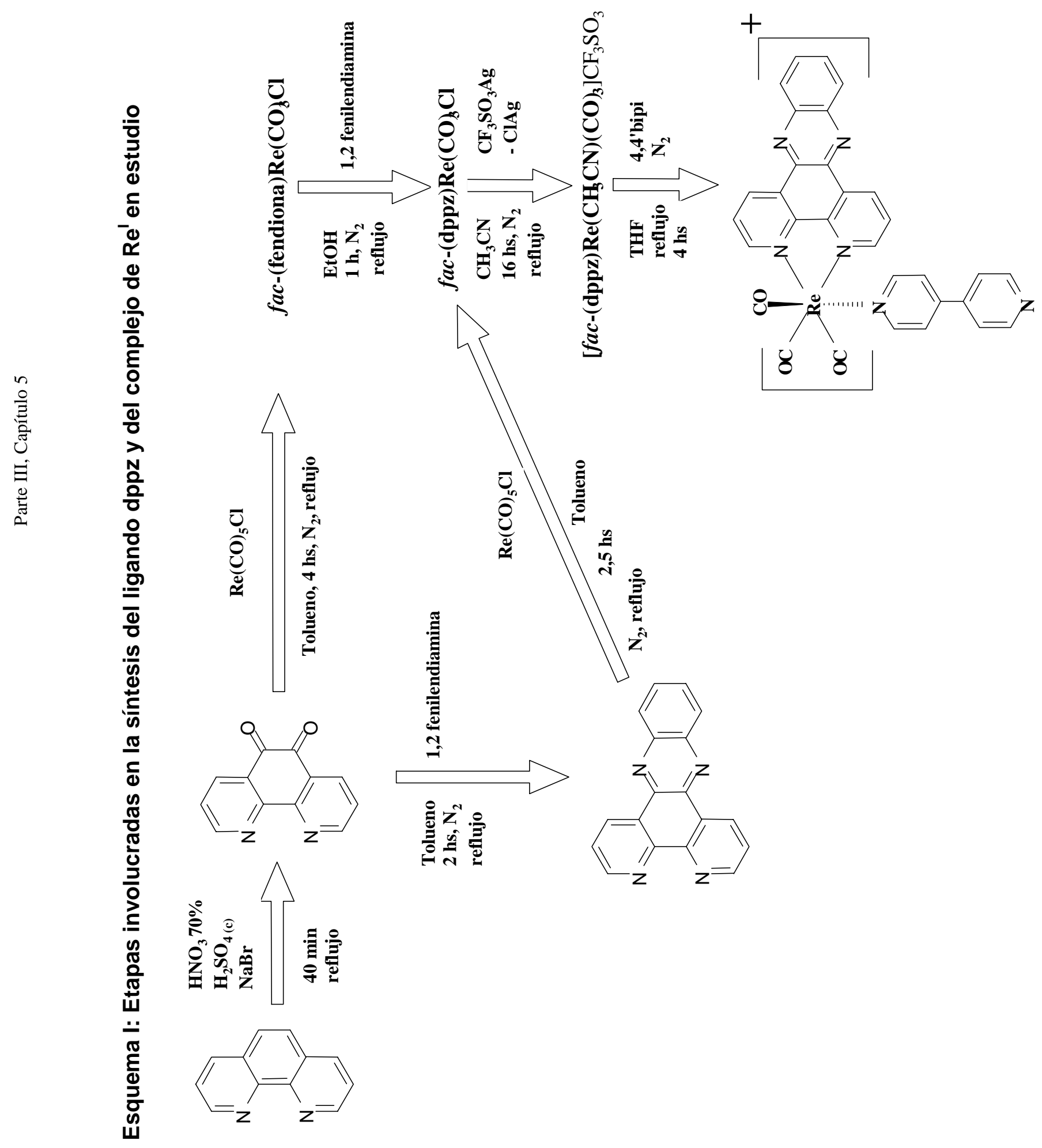




\section{Espectros de absorción infraroja}

En forma similar a lo descripto en el capítulo 2, existen varios ejemplos en la literatura de complejos parientes del complejo de $\operatorname{Re}^{\mathrm{I}}$ en estudio a los cuales se les ha podido determinar su estructura cristalina. En particular, para el $(\mathrm{L}) \operatorname{Re}^{\mathrm{I}}(\mathrm{CO})_{3}(\mathrm{py})^{+}$donde $\mathrm{L}=\mathrm{dppz}$; dppn = benzodipiridofenacina y py = piridina [4], se ha determinado que el $\mathrm{Re}^{\mathrm{I}}$ está en el centro de una estructura octahédrica distorsionada con los grupos carbonilos coordinados en una disposición facial. Del análisis del espectro de absorción IR, figura 1, y ${ }^{1} \mathrm{H}-\mathrm{RMN}$ (ver más adelante), para el (dppz) $\operatorname{Re}^{\mathrm{I}}(\mathrm{CO})_{3}\left(4,4^{\prime} \text { bipi }\right)^{+}$, la interpretación de los mismos indicarían que se adopta una conformación similar. El patrón de bandas observado en la zona de absorción IR de los carbonilos es típica de complejos de $\mathrm{Re}^{\mathrm{I}}$ similares con los $3 \mathrm{C} \equiv \mathrm{O}$ ubicados en la esfera de coordinación de manera facial. La presencia de solo 2 bandas, a 2037 y $1934 \mathrm{~cm}^{-1}$, producto del estiramiento del enlace $\mathrm{CO}$, revelan la mayor simetría observada en este complejo debido a que presenta $3 \mathrm{C} \equiv \mathrm{O}$ y $3 \mathrm{~N}$ piridínicos equivalentes coordinados, siendo, de esta manera menor la distorsión del octahedro para este complejo, que para el $(d p p z) \operatorname{Re}^{\mathrm{I}}(\mathrm{CO})_{3} \mathrm{Cl}$. En éste, se observaron 3 bandas características a 2025, 1924 y $1901 \mathrm{~cm}^{-1}$ [15] certificando la menor simetría debido a la coordinación del Cl.

El espectro IR obtenido para el ligando dppz coincide con el publicado por otros autores [15]. 
Figura 1: Espectro IR de una solución en $\mathrm{ACN}$ del $(\mathrm{dppz}) \operatorname{Re}^{\prime}(\mathrm{CO})_{3}\left(4,4^{\prime} \mathrm{bipi}^{+}\right.$

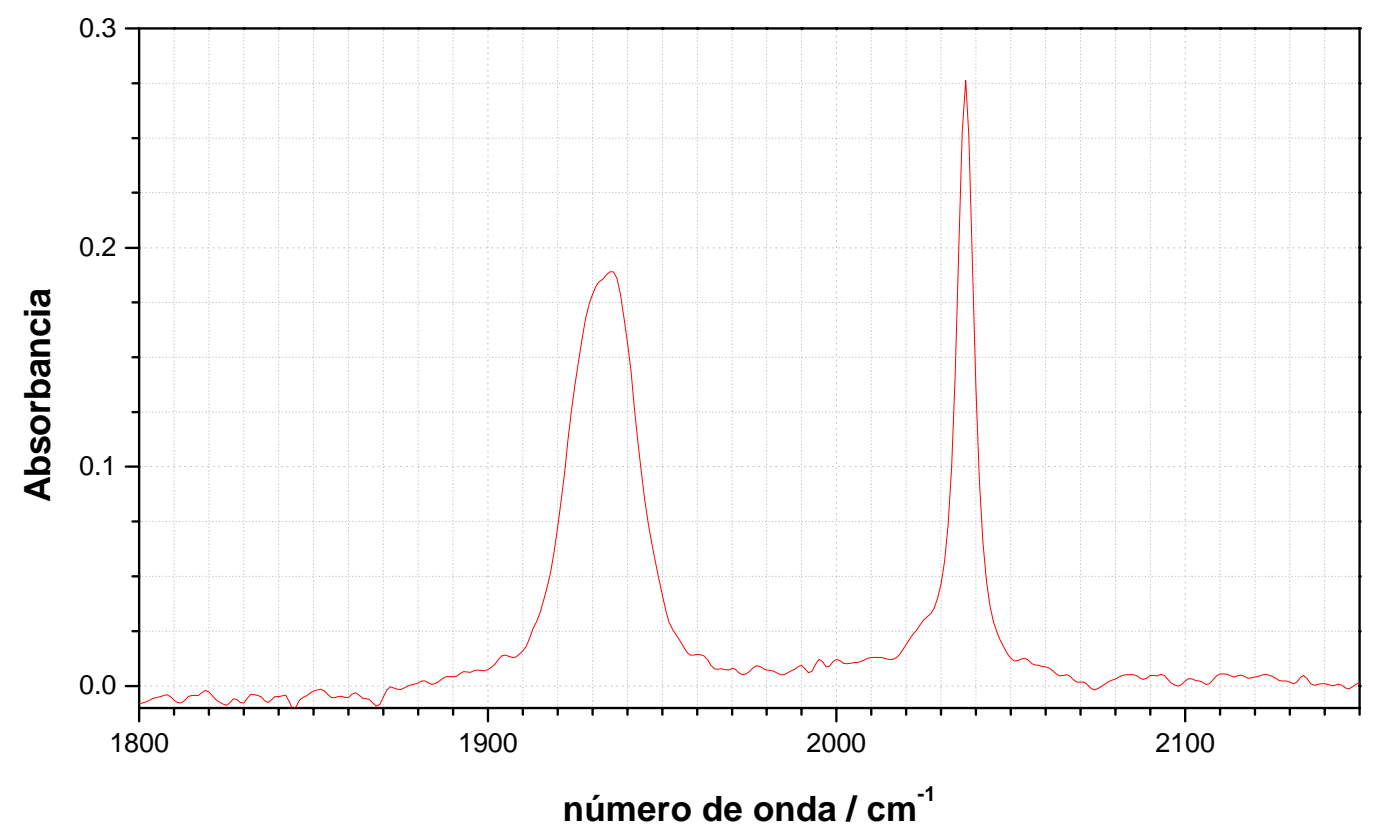

\section{Espectros ${ }^{1} \mathbf{H}-\mathrm{RMN}$}

Los espectros ${ }^{1} \mathrm{H}-\mathrm{RMN}$ del $(\mathrm{dppz}) \operatorname{Re}^{\mathrm{I}}(\mathrm{CO})_{3}\left(4,4^{\prime} \text { bipi }\right)^{+}$y del dppz, se obtuvieron disolviendo la muestra ( $20 \mathrm{mg}$ ) en $0,7 \mathrm{ml}$ de ACN deuterado, aunque solo parte se logró disolver teniendo que filtrar antes de la medición, figuras 2 y 3 . Se obtuvo un buen barrido utilizando un total de aproximadamente 150 pulsos. El registro de los espectros está realizado en valores de desplazamiento $(\delta)$ en ppm, referido a la señal del solvente.

El espectro del $(\mathrm{dppz}) \mathrm{Re}^{\mathrm{I}}(\mathrm{CO})_{3}\left(4,4^{\prime}\right.$ bipi $^{+}{ }^{+}$muestra un grupo de 8 señales intensas con razones de integración de los picos de (1:1:1:2:1:1:1:1), lo que se corresponde con lo esperado para los 18 protones de ambos ligandos en el complejo. Otras señales de menor intensidad podrían corresponder a pequeñas impurezas de mayor solubilidad que el complejo en el solvente utilizado y que se acentúan en nuestras condiciones de trabajo, aunque no se descarta que 
provengan del mismo solvente. Aún así, el espectro refleja un grado de pureza aceptable para este tipo de compuestos.

En la molécula del complejo se encuentran 2 grupos de protones, compuesto por los que corresponden a cada uno de los ligandos que la conforman. A su vez, se generan una serie de subsistemas en relación con las interacciones mutuas que experimentan. Para el ligando dppz existen 2 grupos de protones aislados entre si formados por $(\mathrm{H} 1, \mathrm{H} 2, \mathrm{H} 3)$ y $(\mathrm{H} 4, \mathrm{H} 5)^{2}$. Para la 4,4'bipi, también se encuentran 2 grupos que están formados por (H6, H7) y (H8, H9). En la tabla I se muestra los desplazamientos asignados para cada protón.

Tabla I: Asignación de los desplazamientos protónicos del ligando dppz y del $(\mathrm{dppz}) \operatorname{Re}^{\prime}(\mathrm{CO})_{3}\left(4,4^{\prime} \mathrm{bipi}^{+}\right.$

\begin{tabular}{cccccccccc}
\hline & H1 & H2 & H3 & H4 & H5 & H6 & H7 & H8 & H9 \\
\hline dppz & $\begin{array}{c}\mathbf{9 , 2 0 3} \\
\text { dd }\end{array}$ & $\begin{array}{c}\mathbf{7 , 8 6 7} \\
\text { dd }\end{array}$ & $\begin{array}{c}\mathbf{9 , 5 9 7} \\
\text { dd }\end{array}$ & $\begin{array}{c}\mathbf{8 , 3 6 6} \\
\text { dd }\end{array}$ & $\begin{array}{c}\mathbf{8 , 0 0 4} \\
\text { dd }\end{array}$ & & & & \\
complejo & $\begin{array}{c}\mathbf{9 , 7 0 2} \\
\text { dd }\end{array}$ & $\begin{array}{c}\mathbf{8 , 2 8 6} \\
\text { dd }\end{array}$ & $\begin{array}{c}\mathbf{9 , 8 8 6} \\
\text { dd }\end{array}$ & $\begin{array}{c}\mathbf{8 , 4 3 2} \\
\text { superp. } \\
\text { con H9 }\end{array}$ & $\begin{array}{c}\mathbf{8 , 1 1 8} \\
\text { dd }\end{array}$ & $\begin{array}{c}\mathbf{8 , 5 9 2} \\
\text { dd }\end{array}$ & $\begin{array}{c}7,449 \\
\text { dd }\end{array}$ & $\begin{array}{c}\mathbf{7 , 5 2 6} \\
\text { dd }\end{array}$ & $\begin{array}{c}\mathbf{8 , 4 4 2} \\
\text { dd }\end{array}$ \\
\hline
\end{tabular}

Los subsistemas de la 4,4'bipi presentan señales con las características esperadas para este sistema. Se ven claramente tres señales en el espectro que son doblete doblete (cuatro picos) y una cuarta señal que está superpuesta con la correspondiente a $\mathrm{H}_{4}$. La señal a más bajo campo de estas cuatro, corresponde al protón H6, ya que por estar cerca del centro metálico, queda expuesto a efectos electrónicos y estéricos desprotectores del resto del complejo. La señal acoplada con ésta es aquella que posee el mismo valor de la constante de acoplamiento, J,

\footnotetext{
${ }^{2)}$ Los protones del dppz fueron numerados desde H1 hasta H6 solo una vez ya que por simetría de la molécula son equivalentes con los que no aparecen numerados. En el caso de la 4,4'bipi, los protones se mencionan desde H6 hasta H9 por consideraciones similares (ver estructura de los ligandos, Introducción Parte III)
} 
correspondiendo al protón H7. Las dos señales restantes corresponden entonces al subsistema (H8, H9) y de ambos protones, se presume que la señal para H9 está menos protegida por la cercanía con el nitrógeno no coordinado.

El sistema de los protones del ligando dppz en el complejo presenta interacciones similares a las mostradas cuando está libre y su asignación se basa en lo explicado a continuación.

Figura 2: Espectro ${ }^{1} \mathrm{H}-\mathrm{RMN}$ del (dppz)Re'(CO) ${ }_{3}\left(4,4^{\prime} \text { bipi }\right)^{+}$en $\mathrm{CD}_{3} \mathrm{CN}$

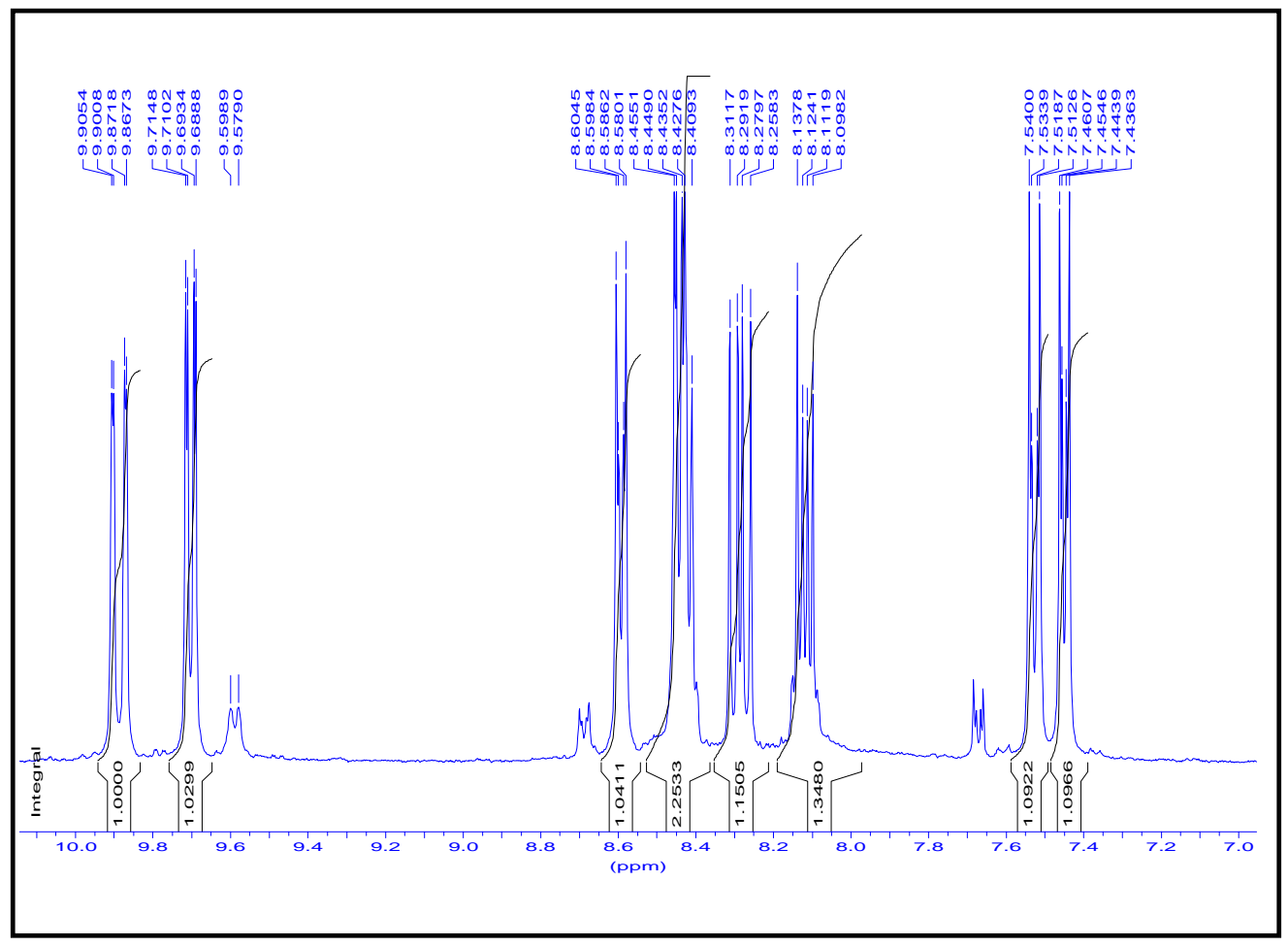

El espectro ${ }^{1} \mathrm{H}-\mathrm{RMN}$ del dppz presenta 5 grupos de señales y las razones de integración (1:1:1:1:1) se corresponde con los 10 protones de la molécula [15].

En el subsistema (H4, H5), la señal desplazada a más bajos campos, fue asignada al protón H4 ya que se encuentra más desprotegido al estar más cerca del nitrógeno (interacción con el par de electrones) y de las corrientes paramagnéticas del resto de los anillos aromáticos. 
El protón H2 del subsistema (H1, H2, H3), fue asignado considerando que solo se acopla a tres enlaces con los protones $\mathrm{H} 1$ y $\mathrm{H} 3$ y por lo tanto los correspondientes valores de $\mathrm{J}$ deben ser grandes. Por el contrario, el acoplamiento a cuatro enlaces entre H1 y H3 debe ser más débil. La señal a más bajo campo fue asignada al protón H3 haciendo consideraciones similares a las planteadas para $\mathrm{H} 4$.

Figura 3: Espectro ${ }^{1} \mathrm{H}-\mathrm{RMN}$ del ligando dppz en $\mathrm{CD}_{3} \mathrm{CN}$

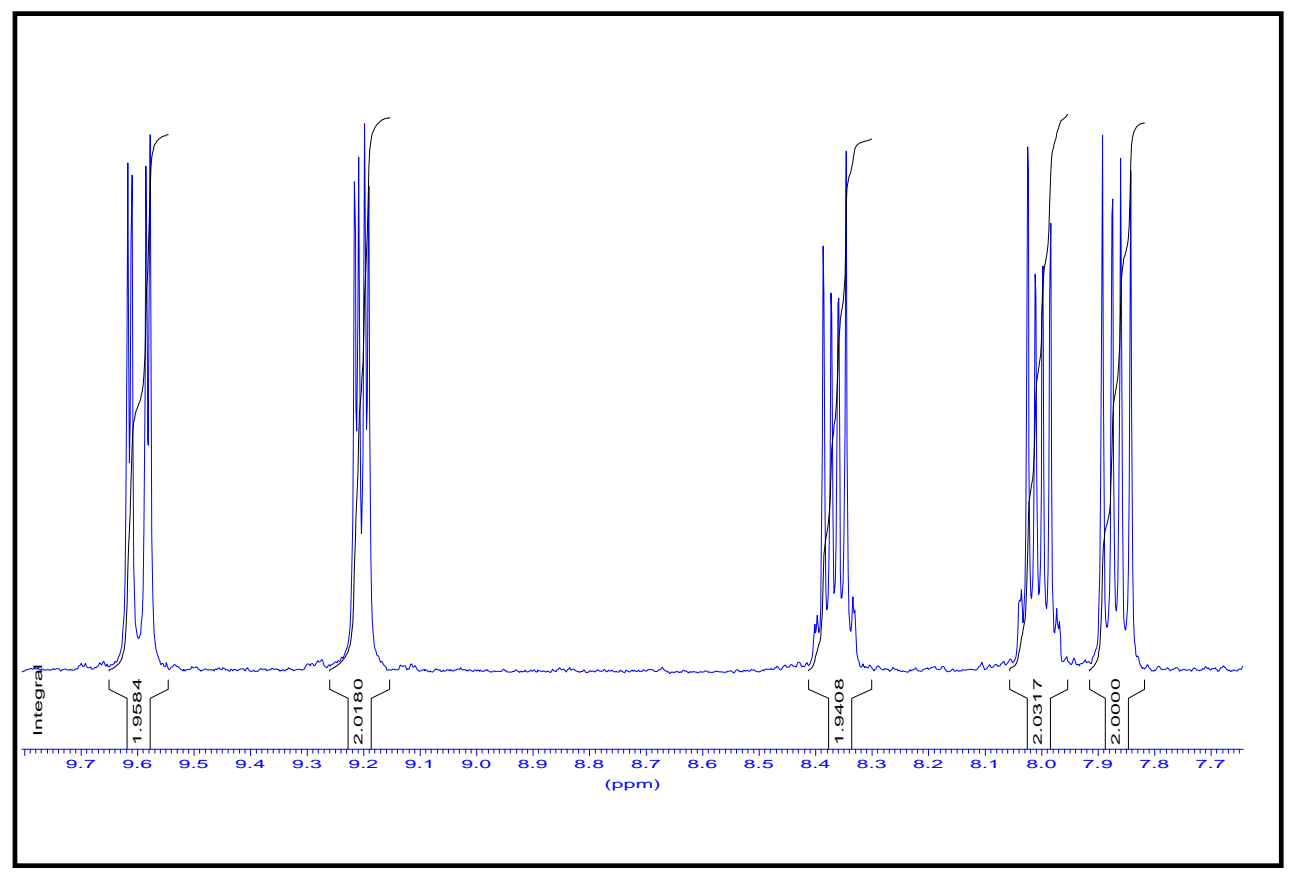




\section{Espectros de absorción UV-visible}

En la figura 4 se observan los espectros de absorción UV-visible del $(\mathrm{dppz}) \operatorname{Re}^{\mathrm{I}}(\mathrm{CO})_{3}\left(4,4^{\prime} \text { 'bipi }\right)^{+}$en $\mathrm{ACN}$ y DCM junto con el espectro del ligando dppz en ACN. En el complejo, pueden verse los rasgos de absorciones compatibles con la de los ligandos que contiene. La banda de menor energía, $\lambda_{\text {máx }} \approx 400 \mathrm{~nm}$ y que se extiende hasta los $500 \mathrm{~nm}$, correspondería a una transferencia de carga $\mathrm{d} \pi\left(\operatorname{Re}^{\mathrm{I}}\right) \rightarrow \pi *(\mathrm{dppz})$. Esta transición es típica de complejos de $\operatorname{Re}^{\mathrm{I}}$ con ligandos bidentados polipiridínicos [12] y no se encuentra en el ligando solo. En la zona hacia energías mayores, $\lambda$ comprendida entre 350 y $400 \mathrm{~nm}$, esta banda se encuentra solapada con los componentes vibrónicos típicos de las transiciones electrónicas del tipo $\pi \rightarrow \pi^{*}$ centradas en el ligando dppz. La zona del máximo de absorción, $\lambda \approx 280 \mathrm{~nm}$, está dominada por este tipo de transiciones las cuales comienzan a solapar, con transiciones IL de la 4,4'bipi, a energías mayores, $\lambda$ entre 200 y 250 nm [16].

Figura 4: Espectros de absorción UV-visible del complejo y del ligando dppz

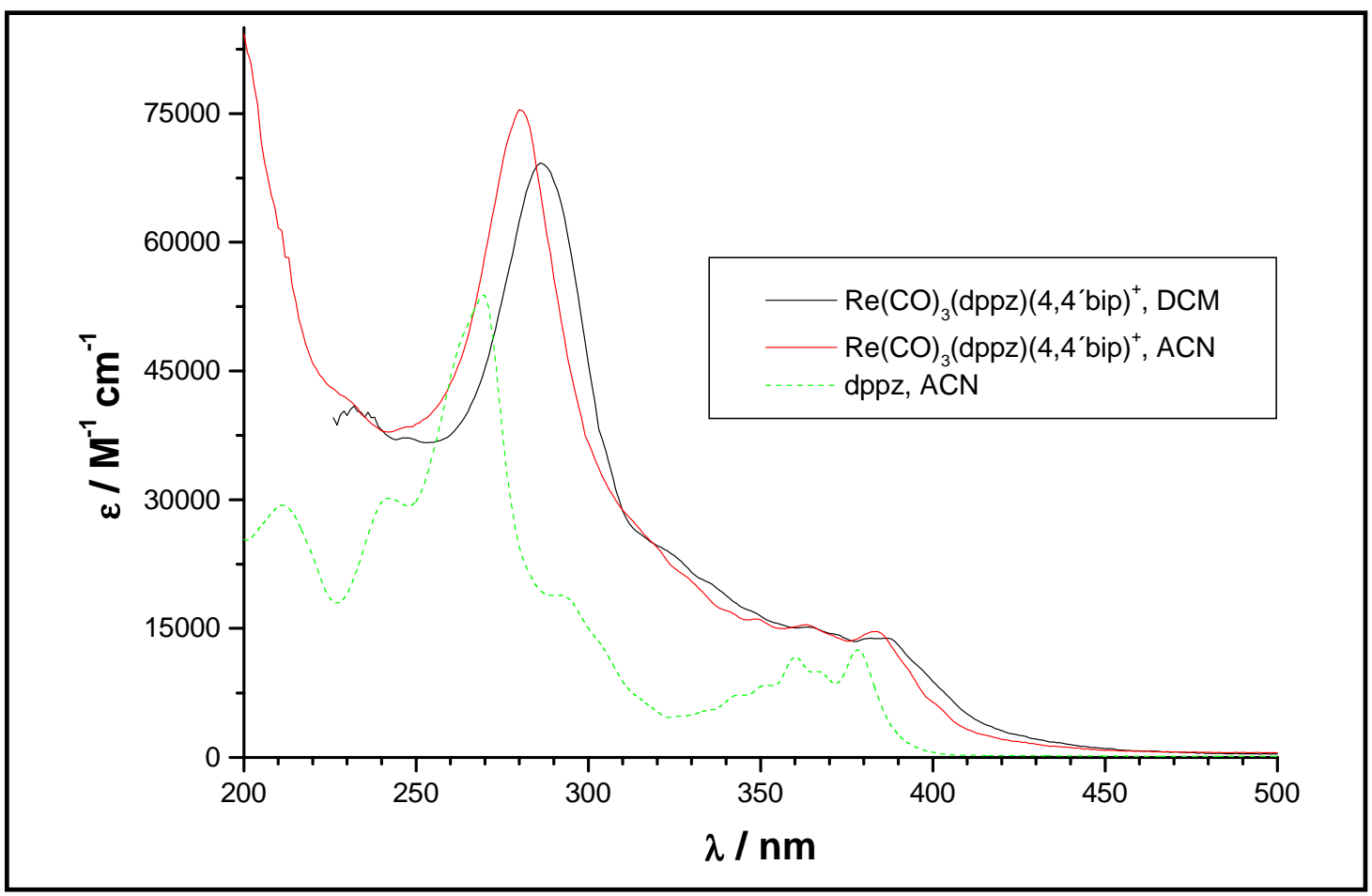


En los capítulos posteriores se analizarán con mayor detalle las transiciones involucradas.

En la tabla II se resumen las bandas de absorción del complejo y del ligando.

Tabla II: Bandas de absorción presentes en el complejo de $\mathbf{R e}^{\prime}$ y en el ligando dppz

\begin{tabular}{|c|c|c|}
\hline Compuesto & $\lambda^{\mathrm{a}} / \mathrm{nm} ; \varepsilon / \mathrm{M}^{-1} \mathrm{~cm}^{-1}$ & Asignación \\
\hline$(\mathrm{dppz}) \operatorname{Re}^{\mathrm{I}}(\mathrm{CO})_{3}\left(4,4^{\prime}{ }^{\prime} \mathrm{bipi}\right)^{+}$ & $\begin{array}{l}\approx 240,38800 \\
281,75267 \\
349,16085 \\
363,15440 \\
370,14294 \\
383,14655 \\
\approx 400,6419 \\
\approx 238^{\mathrm{b}}, 39607 \\
286,69213 \\
348,16921 \\
364,15155 \\
373,14193 \\
387,13890\end{array}$ & $\mathrm{~d} \pi \rightarrow \pi^{*}$ \\
\hline & $\approx 404,7288$ & $\mathrm{~d} \pi \rightarrow \pi^{*}$ \\
\hline
\end{tabular}

dppz

211,29430

242,30187

270,53904

293,18813

343,7230

351,8423

360,11767

367,10004

379,12472

a. Todos los valores corresponden a espectros a temperatura ambiente en acetonitrilo.

b. En diclorometano 


\section{Medidas electroquímicas}

Los potenciales electroquímicos de reducción del (dppz) $\operatorname{Re}^{\mathrm{I}}(\mathrm{CO})_{3}\left(4,4^{\prime} \text { bipi }\right)^{+}$y de los ligandos fueron determinados por voltametría cíclica de alterna. En la figura 5 se muestra el barrido catódico correspondiente a los ligando libres, dppz y 4,4'bipiridina. Para una solución 3,3.10-4 M del ligando dppz, el primer pico de reducción corresponde a un proceso reversible con un $\mathrm{E}_{\mathrm{p}}$ de $-1193 \mathrm{mV}$ medido contra el electrodo de referencia $\mathrm{Ag} / \mathrm{AgCl} / \mathrm{KCl}_{\text {sat }}$, figura 5a. $\mathrm{El}$ barrido anódico no mostró ningún proceso detectable en el intervalo de potencial entre 0 y 1,5 V. El sistema electroquímico de trabajo estuvo compuesto, además, por un electrodo indicador y un contraelectrodo de Pt. Todas las soluciones utilizadas fueron en ACN y medidas con una solución $0,1 \mathrm{M}$ de $\mathrm{NBu}_{4} \mathrm{PF}_{6}$ como electrolito soporte, a $3 \mathrm{mV} / \mathrm{s}$ y con una frecuencia de $6 \mathrm{~Hz}$. En estas condiciones de trabajo, el voltamperograma de una solución 1,4.10 $0^{-4} \mathrm{M}$ de 4,4'bipiridina presenta una onda de reducción reversible e intensa con un $\mathrm{E}_{\mathrm{p}}$ de $-1862 \mathrm{mV}$, figura $5 b$.

En condiciones similares a las descriptas anteriormente, se obtuvo el voltamperograma correspondiente a una solución $1,8 \cdot 10^{-4} \mathrm{M}$ del complejo de $\mathrm{Re}^{\mathrm{I}}$ en estudio cuyos valores de potenciales de media onda permitieron su caracterización desde el punto de vista electroquímico, figura 6. La comparación directa con los potenciales obtenidos para los ligandos por separado, permite observar claramente la excelente concordancia entre el pico de reducción a valores muy negativos de potencial, $\mathrm{E}_{\mathrm{p}}=-1873 \mathrm{mV}$ vs $\mathrm{Ag} / \mathrm{AgCl}$, y el obtenido para la 4,4'bipiridina sola. Por lo tanto, asignamos este pico de reducción a la cupla 4,4'bipi /

\section{4,4'bipi ${ }^{\bullet}$.}


Figura 5: Voltametría cíclica ac de los ligandos a) dppz y b) 4,4’bipi en ACN
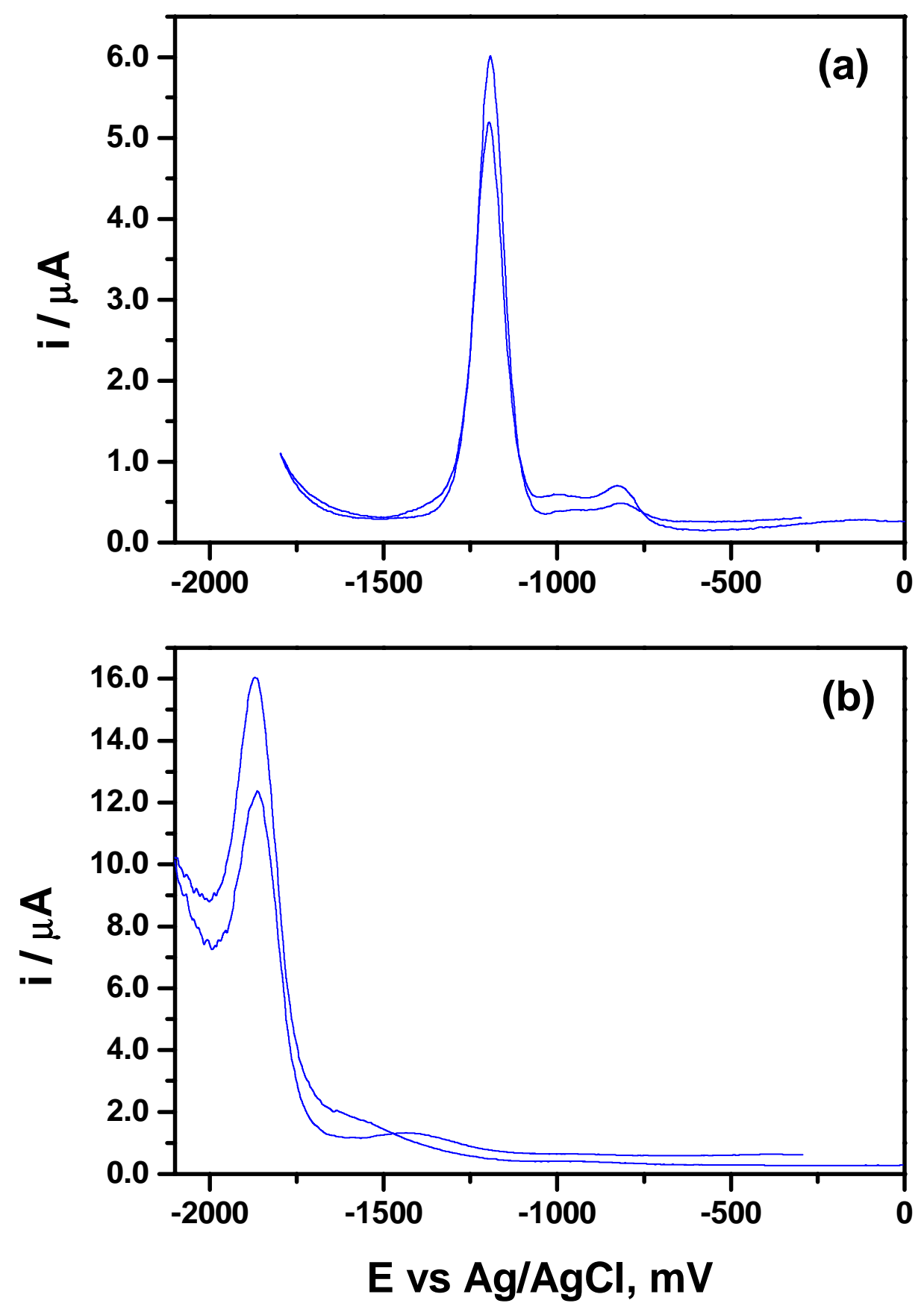

La onda de reducción observada a $-860 \mathrm{mV}$ en el $(\mathrm{dppz}) \mathrm{Re}^{\mathrm{I}}(\mathrm{CO})_{3}\left(4,4^{\mathrm{s}} \text { bipi }\right)^{+}$, se encuentra a potenciales significativamente más altos (menos negativos) comparados con el potencial de 
reducción que se observa en el dppz solo. Es decir, el complejo es más fácil de reducir que el ligando libre.

Por otra parte, en el $(\mathrm{dppz}) \mathrm{Re}^{\mathrm{I}}(\mathrm{CO})_{3} \mathrm{Cl}$ el potencial de reducción se encuentra a $-1,01 \mathrm{~V}$ vs SCE y esta mayor facilidad de reducción es similar a lo observado para complejos con diferentes derivados del dppz [7,15]. Por lo tanto, estamos en condiciones de asegurar que el proceso de reducción a $-0,86 \mathrm{~V}$ está ocurriendo sobre el ligando dppz coordinado. Que los valores de potencial sean menos negativos en los complejos, se debe a que la situación en que se encuentra el ligando cuando está coordinado, permite reacomodar carga con mayor facilidad, por compartir con los orbitales del metal la redistribución electrónica global.

Figura 6: Voltametría cíclica del (dppz) $\operatorname{Re}^{\prime}(\mathrm{CO})_{3}\left(4,4^{\prime} \text { bipi }\right)^{+}$en $\mathrm{ACN}$

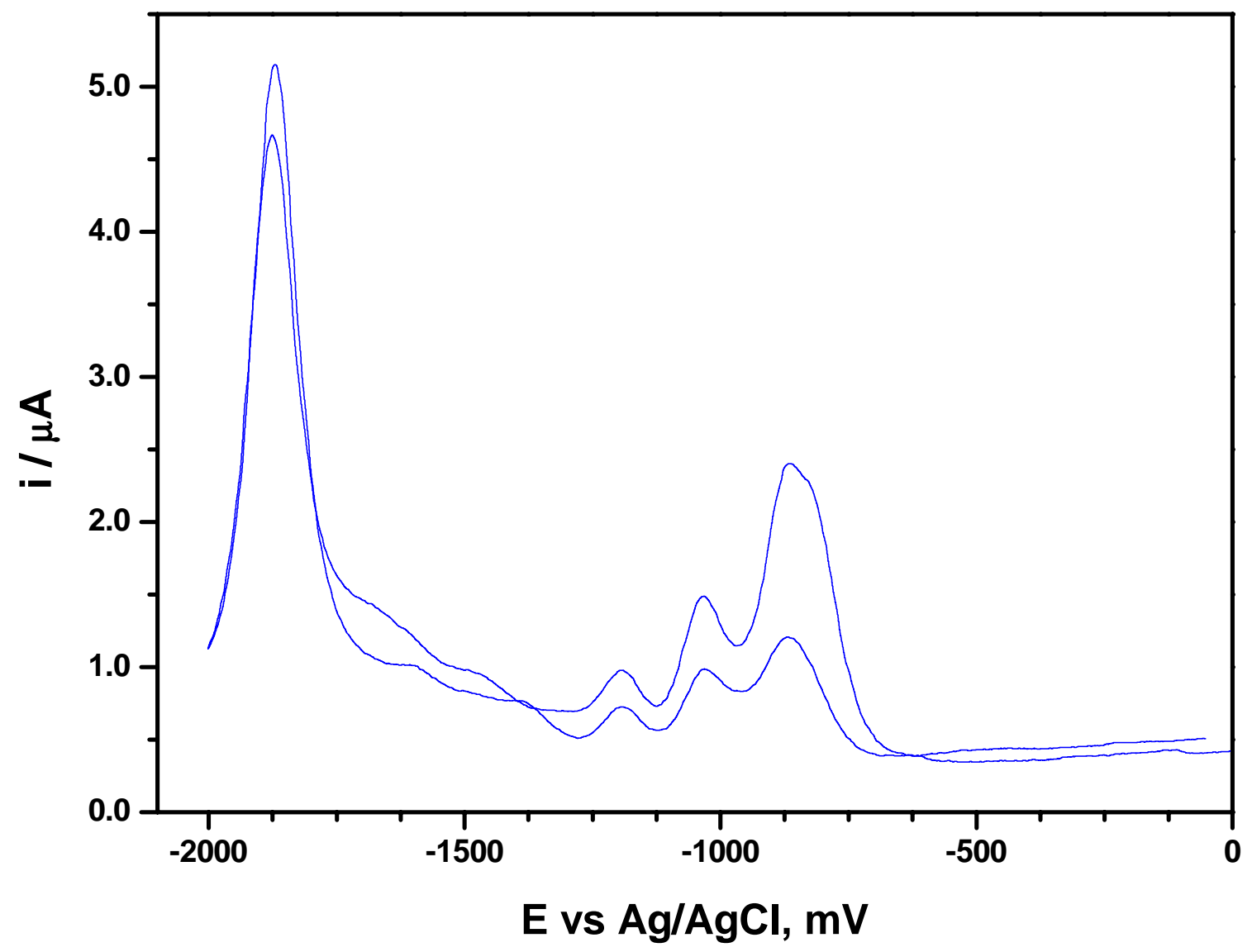


En realidad en la reducción del dppz coordinado se observan tres ondas correspondientes a las distintas posibles ubicaciones del electrón en los diferentes sectores de la estructura molecular del dppz. Muchos trabajos, que se encuentran en la bibliografía, muestran complejos con dppz que presentan este desdoblamiento de la onda de reducción [17-19]. Por otro lado, cálculos teóricos han mostrado que el valor $\mathrm{E}_{1 / 2}=-860 \mathrm{mV}$ corresponde a la reducción del dppz cuando el electrón se encuentra alojado en un orbital molecular antiligante de más baja energía, $\left(b_{1}(p h z)\right)$, ubicado sobre la porción fenacínica del mismo y que sería el responsable de la mayor facilidad para la reducción observada cuando el ligando se encuentra coordinado [18]. Estos estudios teóricos determinaron también que otros orbitales moleculares de baja energía localizados sobre la porción bipiridínica de la molécula del dppz coordinado, $\left(b_{1}(\psi)\right.$ y $\left.a_{2}(\chi)\right)$, pueden, además, alojar otro electrón en procesos de reducción posteriores; fenómeno que aparentemente, no ocurre en la molécula aislada. Las porciones fenacínica y bipiridínica del dppz se refieren al siguiente desdoblamiento imaginario de la molécula del ligando:
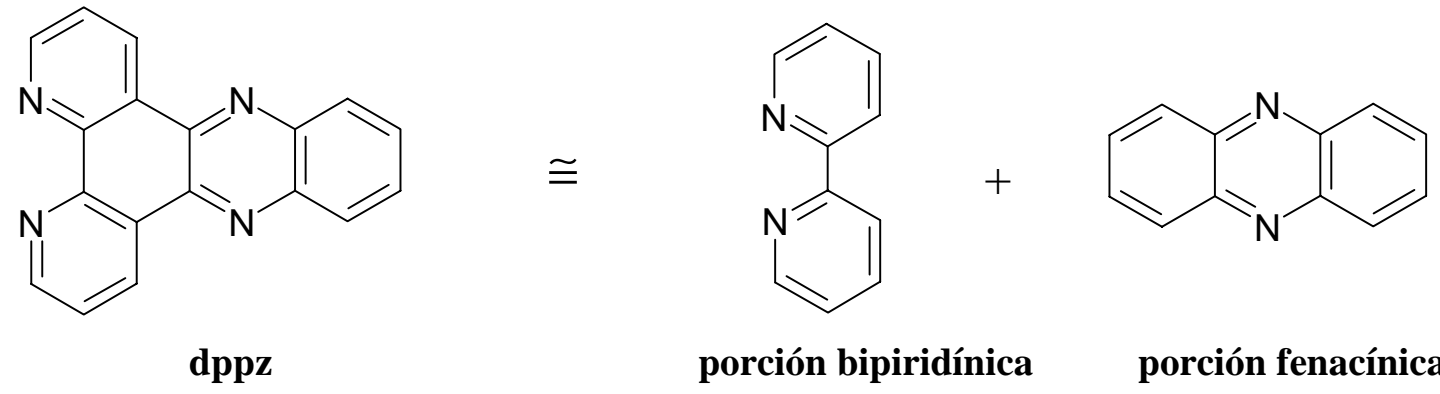

porción fenacínica

En un barrido anódico de potencial en el $(\mathrm{dppz}) \operatorname{Re}^{\mathrm{I}}(\mathrm{CO})_{3}\left(4,4^{\prime} \text { bipi }\right)^{+}$, el pico observado a $+1,45$ V con respecto al $\mathrm{Ag} / \mathrm{AgCl} / \mathrm{KCl}_{\text {sat }}$ fue asignado a la cupla $\mathrm{Re}^{\mathrm{II}} / \mathrm{Re}^{\mathrm{I}}$.

Los resultados de las medidas electroquímicas se muestran en la tabla III 
Tabla III: Potenciales electroquímicos de los ligandos y del complejo de $\mathbf{R e}^{\prime}$

\begin{tabular}{|c|c|c|c|c|c|}
\hline & \multicolumn{5}{|c|}{${ }^{\mathrm{a}} \mathrm{E}_{1 / 2} / \mathrm{V}$ vs $\mathrm{Ag} / \mathrm{ClAg}$} \\
\hline & $+4 \mathrm{e}$ & $+3 \mathrm{e}$ & $+2 \mathrm{e}$ & $+1 e$ & $-1 \mathrm{e}$ \\
\hline 4,4'bipi & & & & $-1,86$ & \\
\hline$(\mathrm{dppz}) \operatorname{Re}^{\mathrm{I}}(\mathrm{CO})_{3}\left(4,4^{\prime}{ }^{\prime} \mathrm{bipi}^{+}\right.$ & $-1,87$ & $-1,19$ & $-1,03$ & $-0,86$ & $+1,45^{\mathrm{b}}$ irr. \\
\hline dppz & & & & $-1,19$ & \\
\hline
\end{tabular}

\section{Conclusiones}

El conjunto de los resultados mostrados en la caracterización del (dppz) $\operatorname{Re}^{\mathrm{I}}(\mathrm{CO})_{3}\left(4,4^{\prime} \text { bipi }\right)^{+}$y del ligando dppz nos revela que el complejo fue obtenido con éxito y con un alto grado de pureza. La conformación de tipo facial para los $3 \mathrm{C} \equiv \mathrm{O}$ presentes en la esfera de coordinación del Re y la presencia del resto de los ligandos se pudo deducir del análisis de los datos de espectroscopía IR, RMN y UV-visible.

Evidencia adicional de la presencia de los ligandos en el complejo de $\operatorname{Re}^{\mathrm{I}}$ obtenido lo aportan los picos de reducción obtenidos por técnicas electroquímicas.

Con el fin de facilitar la interpretación de los resultados que se presentarán en los próximos 2 capítulos, se describen la fotofísica y fotoquímica del ligando dppz y del complejo de $\operatorname{Re}^{\mathrm{I}}$ en estudio, separadamente. La comparación de ambos resultados será utilizada facilitando la identificación de los estados excitados involucrados en el complejo. 
CAPITULO 6

\section{ESTUDIOS FOTOQUÍMICOS Y FOTOFÍSICOS REALIZADOS SOBRE EL LIGANDO DPPZ}




\section{Resultados experimentales y Discusión}

\section{Materiales}

El ligando dppz fue obtenido como se describe en el capítulo 5 y todos los solventes utilizados fueron de calidad pro-análisis. Las aminas alifáticas trietanolamina y trietilamina fueron compradas a Aldrich y se usaron sin posterior purificación. $\mathrm{El} \mathrm{Zn}\left(\mathrm{ClO}_{4}\right)_{2} .6 \mathrm{H}_{2} \mathrm{O}$ fue comprado a Malinckrodt.

\section{Equipos y métodos}

La luminiscencia del ligando dppz en estado estacionario fue obtenida de manera similar a las descriptas en los capítulos 1 y 3 al igual que la determinación de los rendimientos cuánticos de emisión, experimentos de láser flash fotólisis, espectrolectroquímica, etc.

Otros detalles se mencionarán convenientemente en cada caso.

\section{Espectros de absorción y de emisión UV-visible}

Los espectros de absorción UV-visible obtenidos en solventes de diferente polaridad y a temperatura ambiente, se muestran en la figura 1. El espectro consiste en un conjunto de $\sim 13$ bandas mostrando la estructura vibrónica característica en el rango de $\lambda$ s comprendido entre 340 y $380 \mathrm{~nm}$. La primer banda en ACN se encuentra aproximadamente a $26385 \mathrm{~cm}^{-1}$ (379 nm) y concuerda bastante bien con el espectro de absorción descrito recientemente para un compuesto relacionado, el dipirido[3,4-b:2'3'-c]fenazina (I), y cuya banda fue asignada al estado excitado singlete de menor energía ${ }^{1}\left(\pi, \pi^{*}\right)[20]$. 
Trabajando con soluciones concentradas, es posible observar otra banda, de más baja energía, que se extiende hacia $\lambda>400 \mathrm{~nm}$ con un coeficiente de extinción de $650 \mathrm{M}^{-1} \mathrm{~cm}^{-1}$ a $430 \mathrm{~nm}$ $\left(23256 \mathrm{~cm}^{-1}\right)$, insertado de la figura 1 . Esta banda a baja energía y con un valor de $\varepsilon$ pequeño, puede ser inmediatamente atribuida a una transición $S_{o} \rightarrow{ }^{1}\left(n, \pi^{*}\right)$ [21].

El valor calculado para la energía del estado $S_{1}\left(n, \pi^{*}\right)$ para el compuesto relacionado, I, fue de $21284 \mathrm{~cm}^{-1}$. Sin embargo, el valor observado para este compuesto fue de $22800 \mathrm{~cm}^{-1}$ que compara muy bien con el correspondiente al del ligando dppz.

Las absorciones restantes han sido asignadas, fundamentalmente, a transiciones del tipo $\pi \rightarrow \pi^{*}$

Los espectros de emisión obtenidos muestran fuerte dependencia con la $\lambda_{\text {exc }} \mathrm{y}$ con el solvente, a temperatura ambiente, figura 2. En acetonitrilo y metanol los cambios son menores que en diclorometano donde el máximo de emisión se corre $\sim 120$ nm hacia energías menores cuando variamos la longitud de onda de excitación en forma gradual desde 350 a 420 nm. También se verifican cambios con la polaridad del solvente. Sin embargo, para todos los solventes estudiados, se observa el efecto de la longitud de onda de excitación. Es decir, la desaparición de la banda a $\lambda \approx 430 \mathrm{~nm}$ y el crecimiento de una nueva a $\lambda \approx 520 \mathrm{~nm}$, cuando aumenta $\lambda_{\text {exc. }}$. En diclorometano, esta banda de emisión levemente estructurada, aparece centrada en 550 nm. Por otro lado, espectros de emisión tomados a $77 \mathrm{~K}$, en mezclas EtOH/MeOH 4:1 (v/v), no mostraron modificaciones importantes. El comportamiento observado al variar el solvente y la longitud de onda de irradiación sugiere fuertemente la presencia de dos emisiones. 


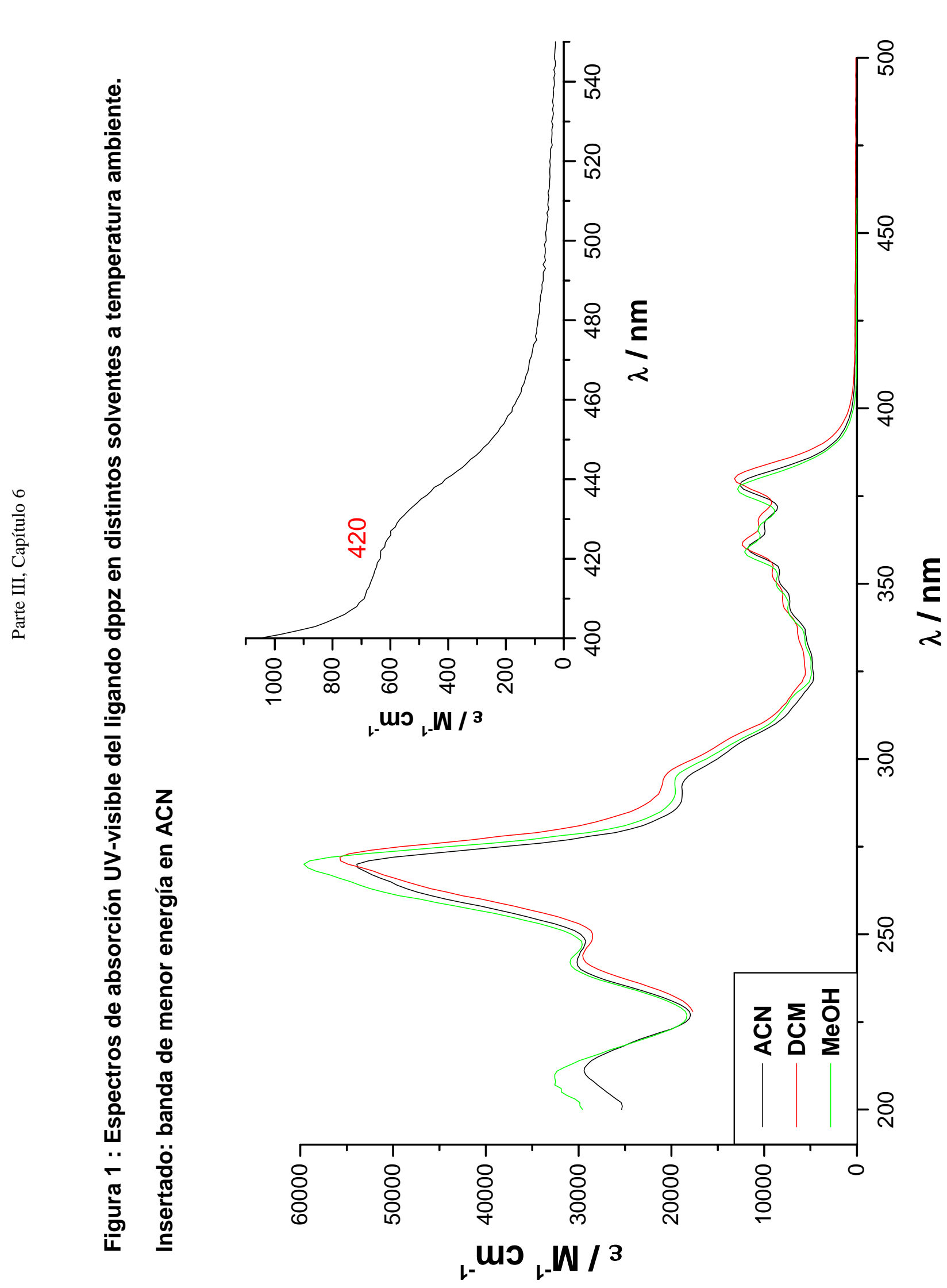




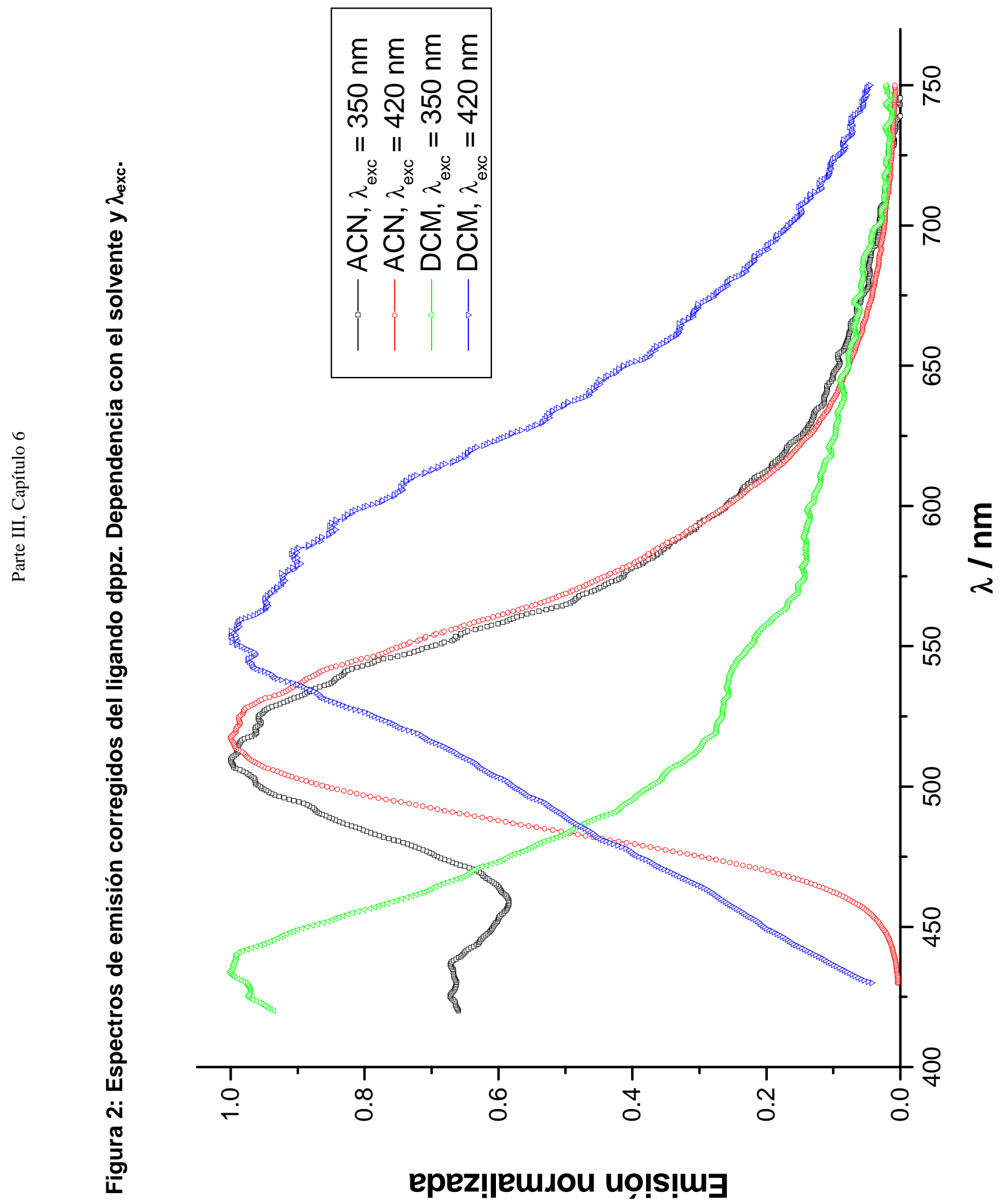


Por otro lado, en la figura 3 se muestra el espectro de excitación, corregido por absorción, del ligando dppz donde puede observarse que la banda activa corresponde a la transición de energía más baja $S_{o} \rightarrow{ }^{1}\left(n, \pi^{*}\right)$. Sin embargo, también se observa otra banda más débil a energías más altas, probablemente correspondiente al estado excitado ${ }^{1}\left(\pi, \pi^{*}\right)$. Estos datos indican que la emisión proviene principalmente del estado excitado ${ }^{1}\left(\mathrm{n}, \pi^{*}\right)$ y los rendimientos cuánticos de emisión mostrados en la tabla I son consistentes con esta observación.

Figura 3: Espectros de excitación del ligando dppz en DCM.

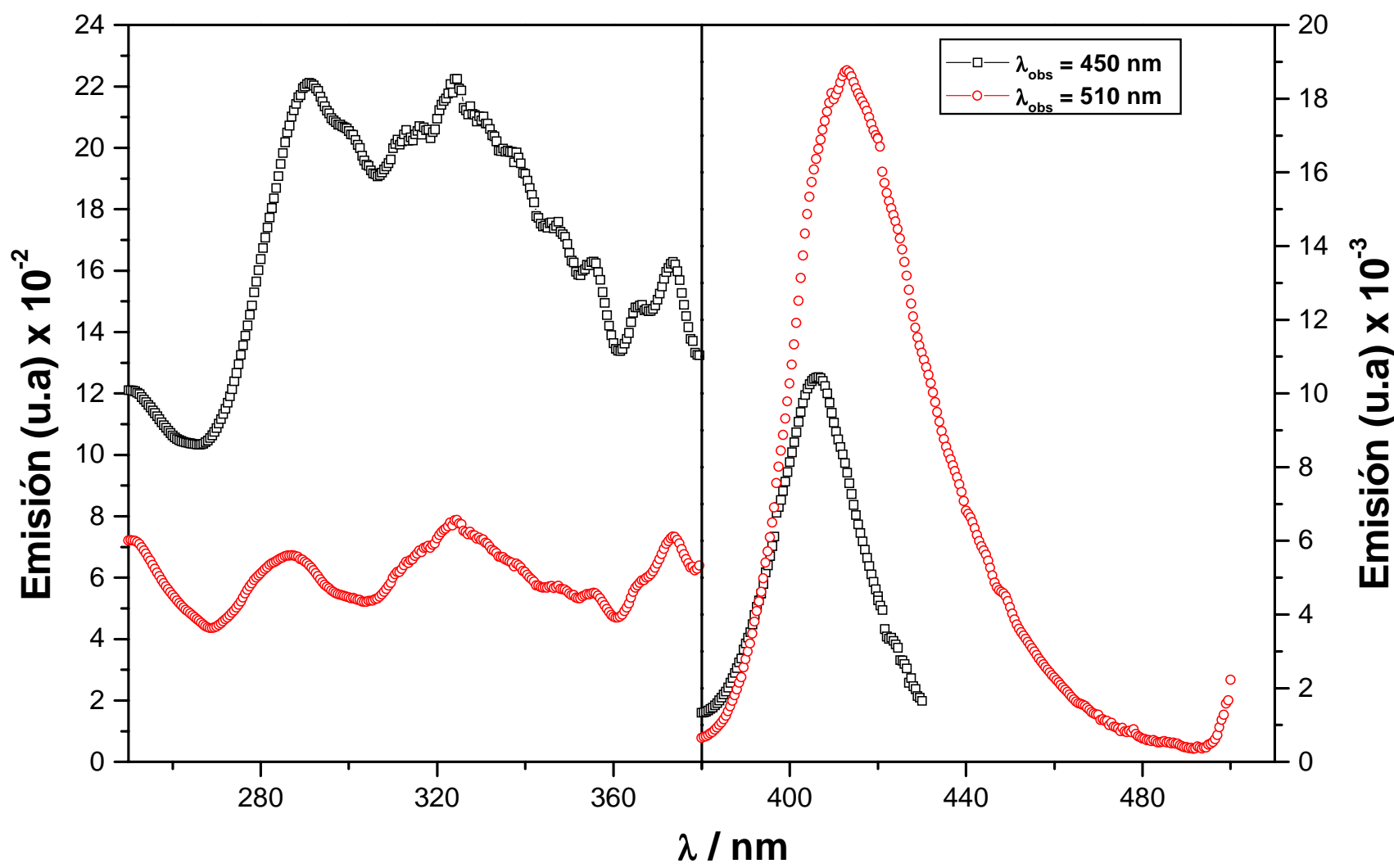


Tabla I: Rendimientos cuánticos de emisión del ligando dppz

\begin{tabular}{ccc}
\hline Solvente & $\phi^{350}$ & $\phi^{400}$ \\
\hline $\mathrm{CH}_{2} \mathrm{Cl}_{2}$ & $2,5 \times 10^{-4}$ & $3,2 \times 10^{-3}$ \\
$\mathrm{CH}_{3} \mathrm{OH}$ & $1,9 \times 10^{-4}$ & $1,8 \times 10^{-2}$ \\
$\mathrm{CH}_{3} \mathrm{CN}$ & $2,3 \times 10^{-4}$ & $1,2 \times 10^{-2}$ \\
\hline
\end{tabular}

En experimentos de fluorescencia flash llevadas a cabo en un equipo PTI $\left(\lambda_{\text {exc }}=337 \mathrm{~nm}\right)$ en ausencia de oxígeno, los tiempos de vida de la emisión, en todos los solventes usados, fueron menores a 15 ns lo que está de acuerdo con la presunción de que la emisión observada, dada su corta vida, pueda atribuirse a la fluorescencia originada en la desactivación de un estado ${ }^{1}\left(\mathrm{n}, \pi^{*}\right)$ y/ó ${ }^{1}\left(\pi, \pi^{*}\right)$.

Sin embargo, dado que no se observó modificación en los $\tau$ al acidificar con $\mathrm{CH}_{3} \mathrm{SO}_{3} \mathrm{H}$ y tampoco se observan diferencias excitando $\lambda=420 \mathrm{~nm}$ (usando el PTI como excitación de una solución metanólica del colorante estilbeno que emite, a su vez, a 420 nm), no fue posible poner en evidencia, con estos experimentos resueltos en el tiempo, la participación del estado ${ }^{1}\left(\mathrm{n}, \pi^{*}\right)$.

\section{Experimentos de láser flash fotólisis}

En las figuras siguientes se muestran los espectros de absorción de las especies transitorias, en solventes de distinta polaridad, generadas con un láser de excímero (XeF) de $351 \mathrm{~nm}$. La concentración de las soluciones fue, en la mayoría de los casos, de 1,2.10 $0^{-4} \mathrm{M}$ y todos los espectros fueron tomados en ausencia de oxígeno. 
En $\mathrm{CH}_{3} \mathrm{CN}$, figura $4 \mathrm{a}$, se observan absorciones entre $375 \mathrm{~nm}$ y $720 \mathrm{~nm}$. Se distinguen claramente tres máximos de absorción a 390, 460 y $550 \mathrm{~nm}$, que decaen en el rango de los microsegundos. La especie transitoria, monitoreada por absorción, desaparece a través de un proceso bi-exponencial $\left(\tau_{1}=2,1 \mu \mathrm{s} ; \tau_{2}=10,1 \mu \mathrm{s}\right)$ observándose una absorción residual (dentro del rango de observación del equipo utilizado) cuyo espectro presenta un máximo a $390 \mathrm{~nm}$.

Experimentos similares llevados a cabo con una solución $2,3.10^{-5} \mathrm{M}$ en $\mathrm{CH}_{2} \mathrm{Cl}_{2}$, mostraron espectros de absorción de la especie transitoria similares a los obtenidos en ACN aunque los decaimientos fueron más lentos $\left(\tau_{1}=2,7 \mu \mathrm{s} ; \tau_{2}=14,6 \mu \mathrm{s}\right.$, respectivamente) y el máximo del producto residual estaba en $400 \mathrm{~nm}$, figura $4 \mathrm{~b}$.

No se observan especies transitorias de vida corta del orden de las que se observan para la emisión. Cuando se fotolizaron soluciones $8,5 \cdot 10^{-5} \mathrm{M}$ del ligando dppz que contenían $\mathrm{Zn}\left(\mathrm{ClO}_{4}\right)_{2} .6 \mathrm{H}_{2} \mathrm{O} 5,3.10^{-2} \mathrm{M}$ en $\mathrm{ACN}$, el espectro de absorción de la especie transitoria producido tenía rasgos similares a los observados en ausencia de $\mathrm{Zn}^{+2}$, con bandas a 390, 460 y $560 \mathrm{~nm}$, figura 5. Sin embargo, es bien notable que la banda de absorción centrada en 390 nm ha sufrido una disminución apreciable con respecto a la banda en $460 \mathrm{~nm}$. En efecto, mientras que en $\operatorname{ACN}$ puro la relación $\operatorname{Abs}(460 \mathrm{~nm}) / \operatorname{Abs}(390 \mathrm{~nm})=0,7$, en presencia de $\mathrm{Zn}^{+2}$ la relación se hace igual a 1,5 debido al decrecimiento de la banda en $390 \mathrm{~nm}$.

Se observa que el decaimiento de la especie transitoria es, también, de tipo bi-exponencial cuando los tiempos de vida se calcularon usando los decaimientos de las trazas correspondientes a $\lambda$ entre 380 y $430 \mathrm{~nm}\left(\tau_{1}=2,7 \mu \mathrm{s} ; \tau_{2}=13,3 \pm 5,2 \mu \mathrm{s}\right)$. Sin embargo, para $\lambda \geq 430 \mathrm{~nm}$, el decaimiento fue mono-exponencial con $\tau=3,8 \mu \mathrm{s}$. 
Figura 4: Espectros de absorción de la especie transitoria obtenida irradiando con pulsos de luz de $351 \mathrm{~nm}$ soluciones deaereadas del ligando dppz:

a) $1,2 \cdot 10^{-4} \mathrm{M}$ en $\mathrm{ACN}$ y b) $2,3 \cdot 10^{-5} \mathrm{M}$ en $\mathrm{DCM}$.
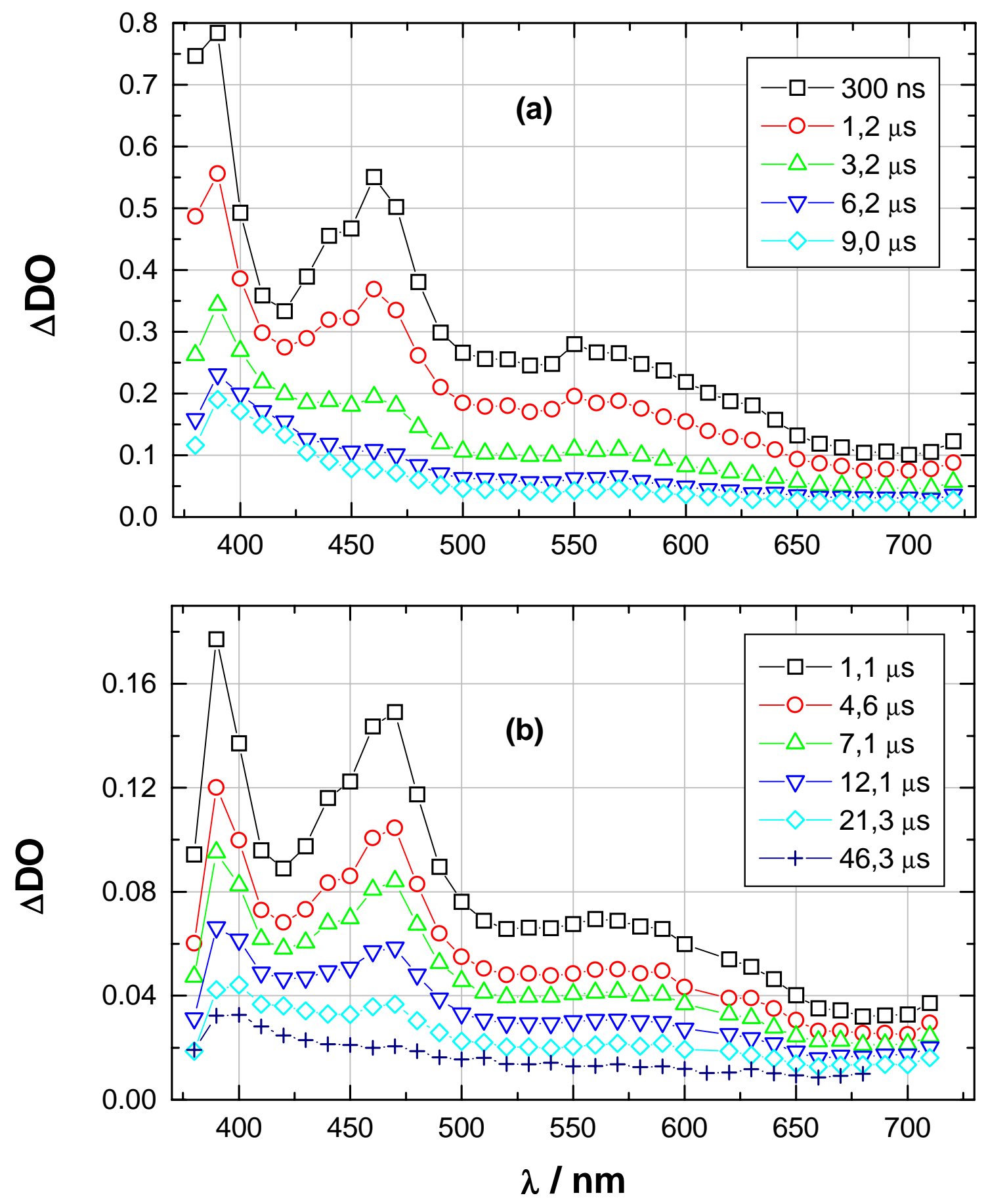
Figura 5: Espectros de absorción de las especies transitorias obtenidas irradiando, con pulsos de luz de $351 \mathrm{~nm}$, soluciones deaereadas del ligando dppz 8,5.10 $0^{-5} \mathrm{M}$ conteniendo $\mathrm{Zn}^{+2} 5,3 \cdot 10^{-2} \mathrm{M}$ en $\mathrm{ACN}$.

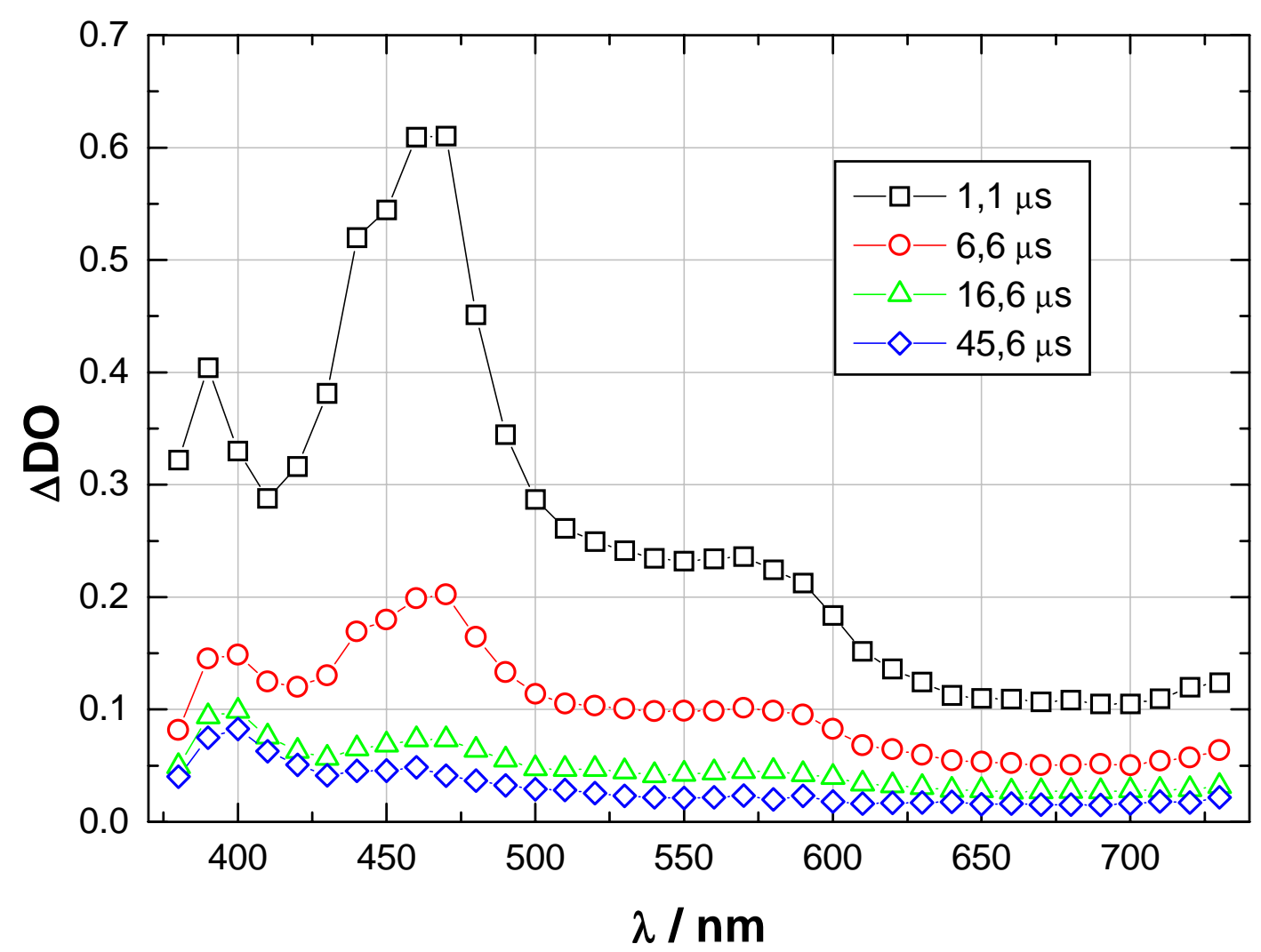

En concordancia con lo sugerido en la literatura [5-7], las absorciones observadas en los experimentos precedentes corresponderían a dos posibles estados excitados del dppz: los tripletes ${ }^{3}\left(\mathrm{n}, \pi^{*}\right) \mathrm{y}^{3}\left(\pi, \pi^{*}\right)$, respectivamente. El máximo de absorción en $\sim 460 \mathrm{~nm}$ es asignada como característica del estado ${ }^{3}\left(\pi, \pi^{*}\right)$ ya que se observa también en el espectro del estado triplete de la fenacina $[5,22]$. Por lo tanto, cuando se introduce el ión $\mathrm{Zn}^{2+}$, éste coordina con los nitrógenos de la porción bipiridínica del dppz impidiendo algunas transiciones $n \rightarrow \pi^{*}$. 
Considerando esta observación, se puede inferir que la banda de absorción de las especies transitorias, centrada en los $390 \mathrm{~nm}$, estaría relacionada con el estado ${ }^{3}\left(\mathrm{n}, \pi^{*}\right)$ lo cual explica que cuando nos alejamos de ese máximo de absorción, en el análisis cinético de las trazas de los experimentos en presencia de $\mathrm{Zn}^{2+}$, sólo se observe el estado ${ }^{3}\left(\pi, \pi^{*}\right)(\tau=3,8 \mu \mathrm{s})$.

Sin embargo, otra posibilidad sería que dichos espectros correspondieran a algún intermediario de reacción. Con el objetivo de determinar la reactividad de estas especies transitorias, se realizó un experimento en ausencia de oxígeno con soluciones $1,2.10^{-4} \mathrm{M}$ del ligando dppz, en acetonitrilo, conteniendo 2-propanol 0,93 M, figura 6.

Figura 6: Espectros de absorción de la especie transitoria obtenida irradiando con pulsos de luz de $351 \mathrm{~nm}$ soluciones deaereadas del ligando dppz $1,2.10^{-4} \mathrm{M}$ conteniendo 2-PrOH 0,93 M en ACN

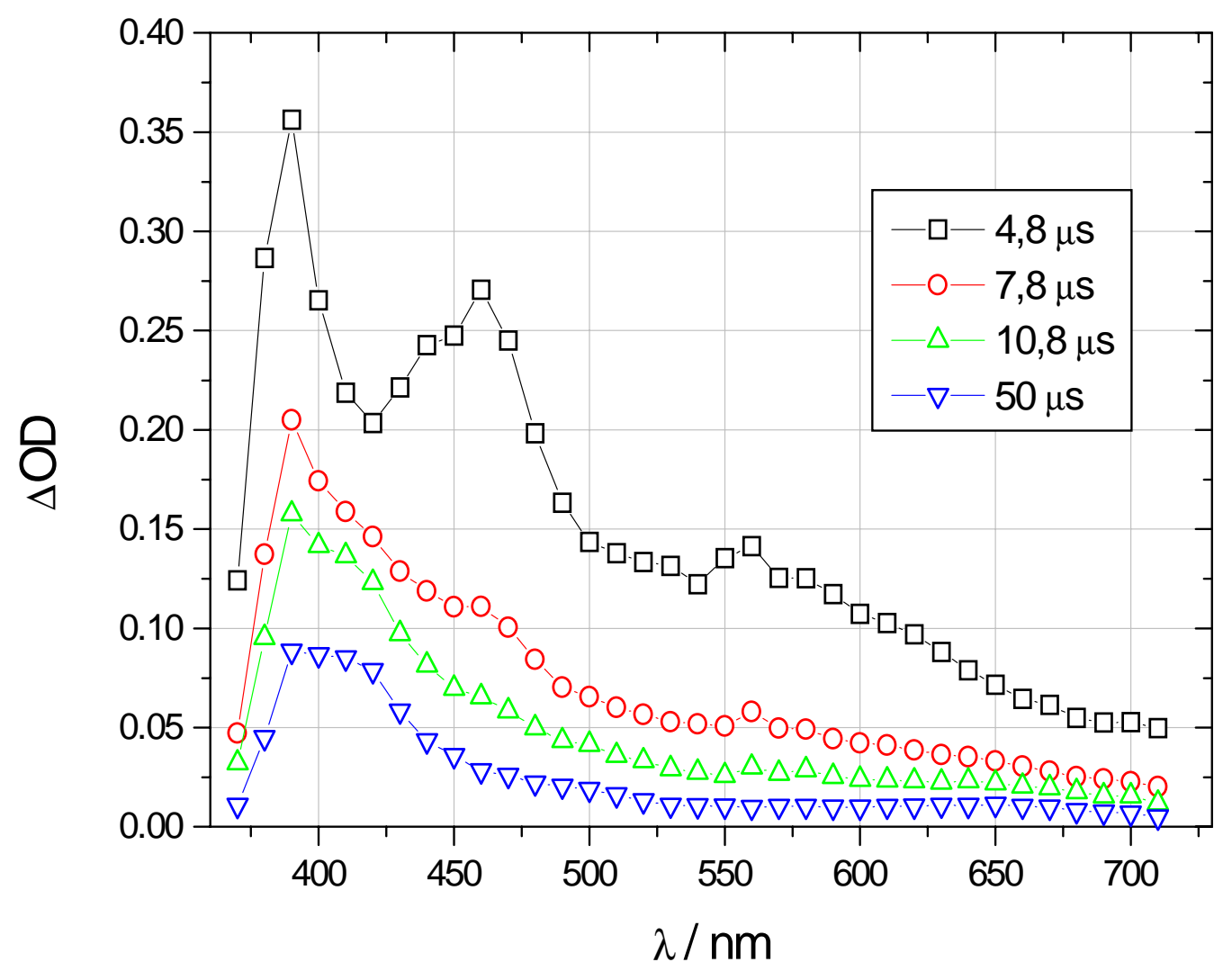


Es conocido que este alcohol es un fuerte donor de $\mathrm{H}$ y por lo tanto cabría esperar una reacción de abstracción de $\mathrm{H}$ con alguno de los estados tripletes involucrados. Sin embargo, el espectro de la especie transitoria obtenido, no presenta mayores diferencias con respecto al espectro en ACN.

Por otra parte, experimentos similares llevados a cabo con soluciones en acetonitrilo del dppz que contenían, además, trietanolamina $0,93 \mathrm{M}$ aportaron evidencia concreta sobre la reactividad de uno de los estados involucrados.

El espectro inicial, figura 7 a, carece de los rasgos atribuidos al estado ${ }^{3}\left(\pi, \pi^{*}\right)$, lo cual indicaría que este ha reaccionado con la TEOA. Se observa, por lo tanto, el decaimiento de la banda de absorción con máximo a $400 \mathrm{~nm}$, atribuible al ${ }^{3}\left(\mathrm{n}, \pi^{*}\right)$, en forma mono-exponencial $(\tau=18$ $\mu \mathrm{s})$, hasta un producto con máximo de absorción en esa misma longitud de onda.

En principio, la posibilidad de abstracción de hidrógeno de la TEOA por alguno de los tripletes no debería ser descartada. Sin embargo, la ausencia de tal reacción con el 2-propanol obliga a descartarla. Además, los estados ${ }^{3}\left(n, \pi^{*}\right)$ son los que generalmente están involucrados en este tipo de reacciones y siendo la energía de estos estados, en este caso, suficientemente baja es posible que ello sea la causa de que la reacción no ocurra.

Las aminas como la TEOA son buenos donores de electrones y, en tal sentido, hay mucha información sobre la reducción de estados excitados por aminas, para dar un radical cargado negativamente y la especie oxidada de la amina. Es decir, una reacción bi-molecular de transferencia de electrones. En nuestro caso, el espectro residual de absorción mostrado en la figura 7a debería corresponder a la especie dppz ${ }^{\bullet-}$, consistente con :

$\left(\mathrm{n}, \pi^{*}\right) \mathrm{y} / \mathrm{o}^{3}\left(\pi, \pi^{*}\right) \mathrm{dppz} \quad+\mathrm{TEOA} \longrightarrow \mathrm{TEOA}^{+}+\mathrm{dppz}^{\bullet}$ 
Figura 7: a) Espectros de absorción de las especies transitorias obtenidas irradiando, con pulsos de luz de $351 \mathrm{~nm}$, soluciones deaereadas del ligando dppz $1,2 \cdot 10^{-4} M$ conteniendo TEOA 0,93 M en ACN y b) Espectro del radical dppz ${ }^{\circ}$ electrogenerado, en ACN
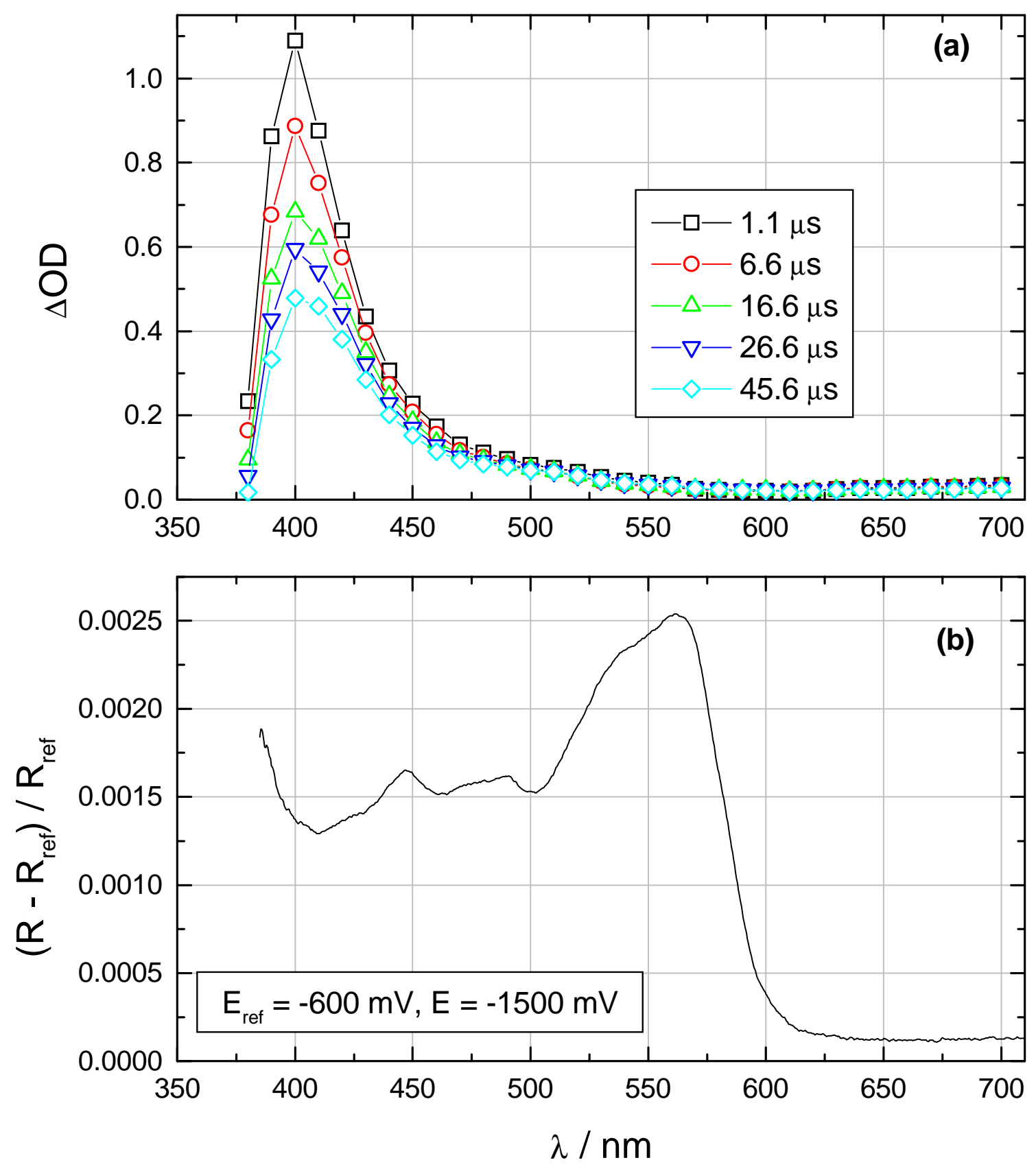
Con el objetivo de confirmar esta proposición, se llevaron a cabo experimentos de espectroelectroquímica de alta sensibilidad, para obtener el espectro de reflectancia relativa, $\Delta \mathrm{R} / \mathrm{R}, \mathrm{UV}$-visible de la especie reducida $\mathrm{dppz}^{\bullet-}$ generada electroquímicamente, modulando el potencial entre -600 y $-1500 \mathrm{mV}$ vs $\mathrm{Ag} / \mathrm{AgCl} / \mathrm{KCl}_{\text {sat. }}$, es decir, entre un potencial anterior y otro posterior a la onda de reducción (ver capítulo 5, figura 5), a $11 \mathrm{~Hz}$ y en un sistema electroquímico compuesto por $\mathrm{Pt} / \mathrm{ACN} / 0,1 \mathrm{M} \mathrm{Bu}_{4} \mathrm{NPF}_{6}{ }^{3)}$.

Sorprendentemente el espectro obtenido, figura $7 \mathrm{~b}$, presenta pocas similitudes con el espectro de la figura 7a, lo que en principio descartaría la posibilidad de que la reacción con TEOA sea de transferencia de electrones. Cabe destacar que el espectro mostrado en la figura $7 \mathrm{~b}$ obtenido en nuestro laboratorio coincide plenamente con el publicado por otros autores en diferentes trabajos $[7,15,18,19]$.

Es probable que la discrepancia entre los espectros se deba a la naturaleza estructural del dppz y a la consecuente existencia de varios orbitales desocupados de energías similares capaces de recibir un electrón en el proceso de reducción, lo cuál daría lugar a la posibilidad de obtener radicales $\mathrm{dppz}^{\bullet-}$ con características espectrales diferentes. Sobre este tema se han hecho algunas consideraciones en el capitulo 5. Básicamente, en la bibliografía se ha planteado la existencia de orbitales "redox" y orbitales "ópticos" en donde se alojaría el electrón dependiendo en qué condiciones ocurre la reducción. La participación de diferentes orbitales en un proceso fotoquímico de reducción frente a la reducción electroquímica podría justificar las diferencias observadas en los espectros $[18,19]$.

\footnotetext{
3) $\Delta \mathrm{R} / \mathrm{R}=\left(\mathrm{R}-\mathrm{R}_{\mathrm{ref}}\right) / \mathrm{R}_{\mathrm{ref}}$, donde $\mathrm{R}=$ reflectancia a un potencial $\mathrm{E}=-1,5 \mathrm{~V}$ y $\mathrm{R}_{\mathrm{ref}}=$ reflectancia de referencia a $\mathrm{E}_{\text {ref }}=-0,6 \mathrm{~V}$
} 


\section{Experimentos de fotólisis estacionaria}

Con el objetivo de caracterizar los posibles productos de reacción generados durante la fotólisis, se irradió con luz de $350 \mathrm{~nm}\left(\mathrm{I}_{0}=6,5.10^{-4}\right.$ Einstein $\left.\mathrm{l}^{-1} \mathrm{~min}^{-1}\right)$ una solución deaereada del ligando dppz en ACN, siguiendo los cambios en los espectros de absorción UV-visible a distintos tiempos de fotólisis. Esta solución fue lo suficientemente concentrada como para asumir la absorción total de la luz incidente durante todo el experimento. Los resultados de la fotólisis estacionaria muestran la formación de foto-productos luego de 106 minutos de irradiación. Si el mismo experimento se lleva a cabo adicionando un $20 \%$ de 2-propanol, se observan cambios espectrales similares pero mayores indicando un incremento en la cantidad de los productos formados. En la figura 8 se muestran los espectros diferencia, $\Delta \mathrm{A}$, en las dos condiciones de fotólisis. Estos espectros se obtuvieron haciendo la diferencia entre el espectro de absorción de la solución fotolizada a tiempo final y el de la solución sin fotolizar.

Los cambios espectroscópicos observados aquí sugerirían que el 2-PrOH favorece la formación de los productos. Sin embargo, como hemos visto en los experimentos de fotólisis flash, no se espera una reacción con el solvente.

Con el propósito de analizar la naturaleza de los productos se realizaron experimentos similares pero a escala preparativa irradiando soluciones adecuadas durante más de 14 horas. Las soluciones irradiadas fueron analizadas por espectroscopía IR, CG-masa y HPLC. Con ninguno de estos procedimientos fue posible detectar productos dentro de los límites de detección de las técnicas utilizadas. Estos resultados sugerirían que la transformación fotoquímica observada posee un rendimiento cuántico muy bajo. La naturaleza del producto residual observado en la escala de tiempo del láser se ha propuesto, en la literatura, que puede ser del tipo de un isómero de valencia del dppz [5]. Los resultados obtenidos no permiten avalar esta hipótesis. Otros experimentos serán necesarios llevar a cabo para verificar esta 
presunción. De todas maneras, de lo observado en los experimentos de flash fotólisis se puede inferir que, de ocurrir lo anterior, las especies intermediarias generadas, desaparecen regenerando el reactivo original.

Figura 8: Fotólisis estacionaria irradiando con luz de $350 \mathrm{~nm}$ soluciones deaereadas del ligando dppz: espectros diferencia

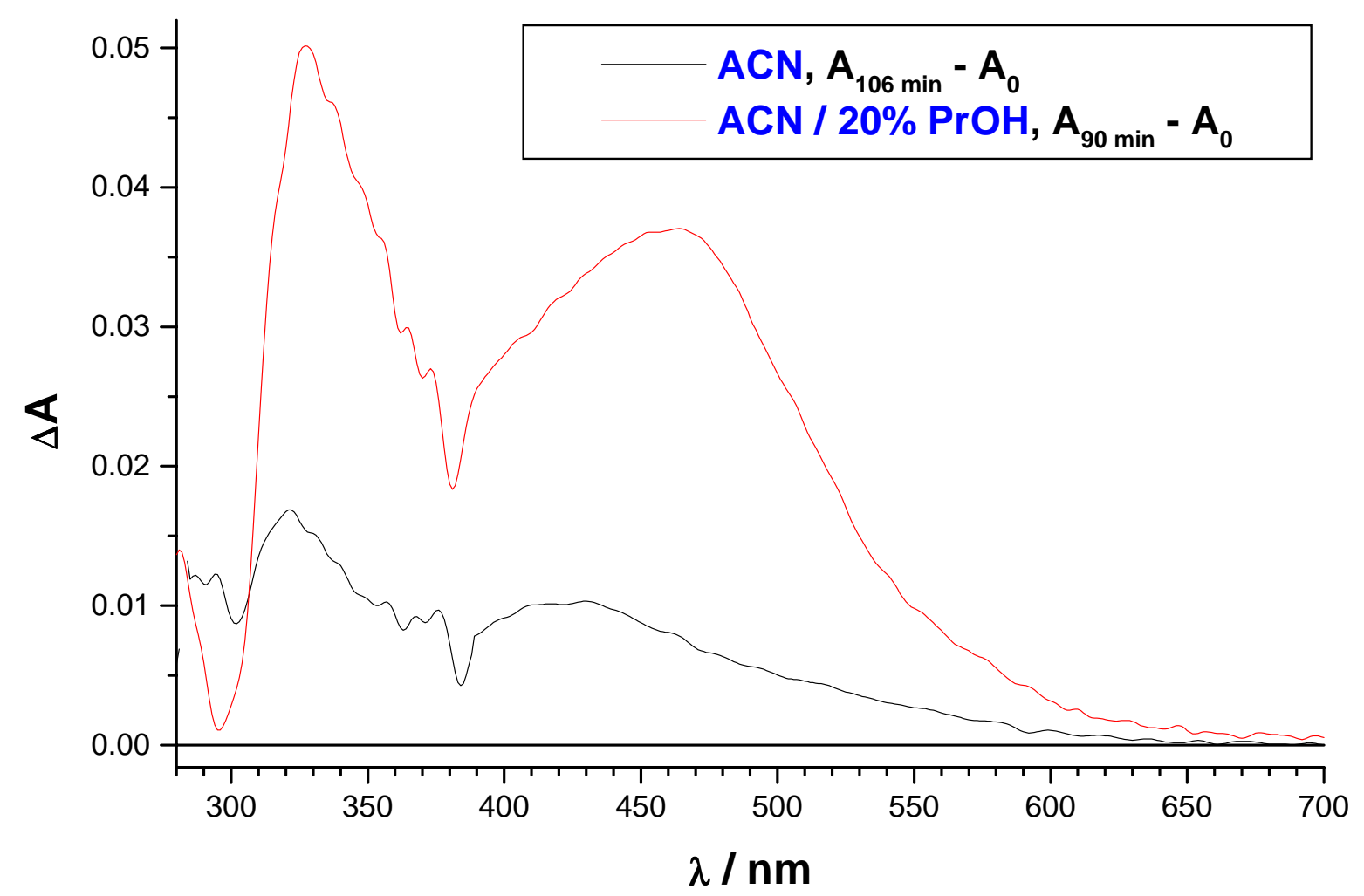




\section{Conclusiones}

Los resultados obtenidos pueden resumirse en las siguientes conclusiones:

$3 / 4 \square$ cuando el ligando dppz se irradia con energías comprendidas entre $33.300 \mathrm{~cm}^{-1}$ y 22.200 $\mathrm{cm}^{-1}$ se pueblan los estados singletes ${ }^{1}\left(\mathrm{n}, \pi^{*}\right)$ y ${ }^{1}\left(\pi, \pi^{*}\right)$ que desaparecen por dos vías:

a) retornando al estado fundamental emitiendo luz (fluorescencia)

b) poblando por cruzamiento intersistemas los tripletes correspondientes, que a su vez regresan al estado fundamental por desactivación no radiativa.

De las observaciones realizadas se puede inferir que este comportamiento es posible si los estados excitados $n \pi^{*}$ y $\pi \pi^{*}$ no están conectados entre sí dando lugar a dos conjuntos independientes. La energía relativa de los estados involucrados se puede representar, en forma aproximada, en el siguiente diagrama de Jablonski donde las flechas azules representan caminos radiativos y las rojas a los no radiativos:

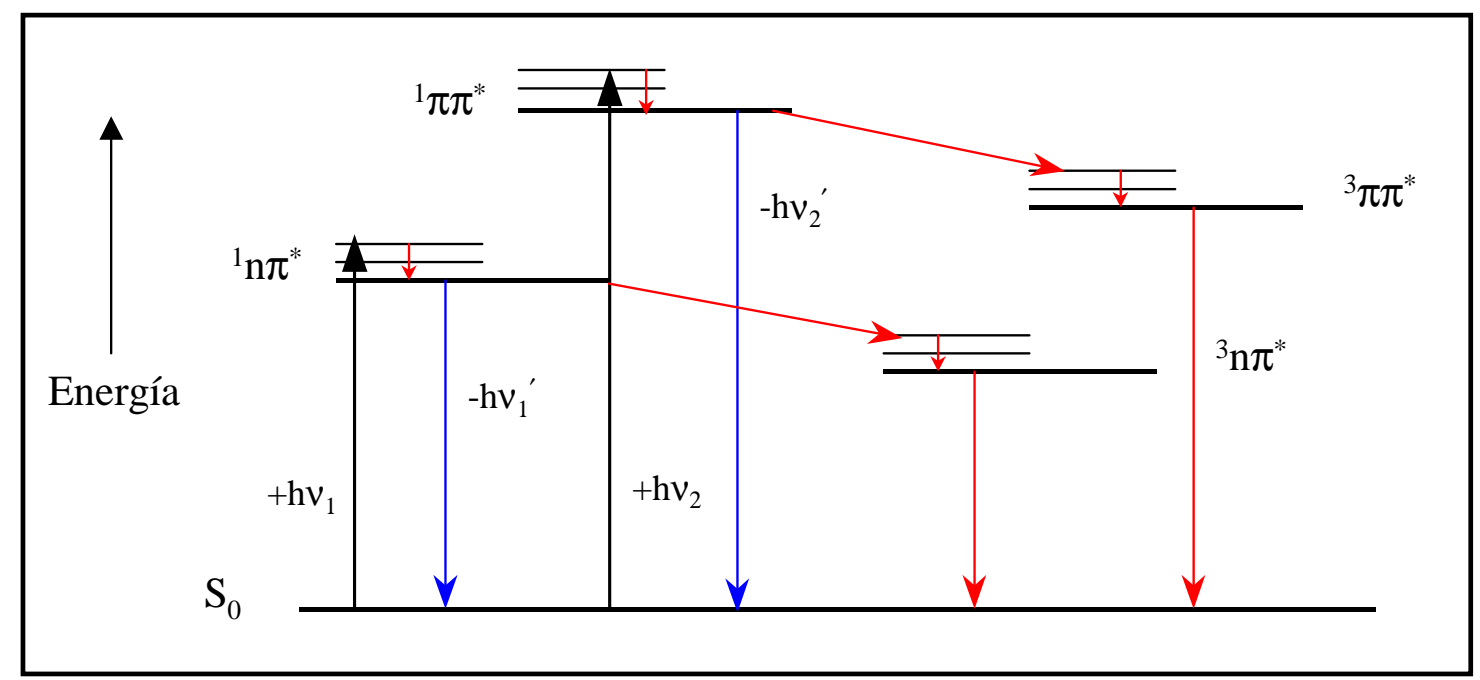

$3 / 4 \square$ los estados excitados tripletes del dppz pueden reaccionar con aminas reductoras en una reacción bimolecular de transferencia de electrones dando lugar a la formación del radical $\mathrm{dppz}^{\bullet}$. El espectro de esta especie así obtenido no se asemeja al espectro del radical obtenido 
electroquímicamente. Esta discrepancia puede ser racionalizada teniendo en cuenta la existencia de orbitales "redox" y orbitales "ópticos".

3/4 $\square$ la reactividad de estos estados excitados frente a reacciones de abstracción de $\mathrm{H}$ no ha podido ser verificada. Esto es coincidente con estudios previos realizados en solventes con distinta capacidad donora de $\mathrm{H}$ [5]. Aunque una reacción de este tipo podría justificar los pequeños cambios fotoquímicos observados en los experimentos de fotólisis estacionaria, tal reacción ocurriría con un rendimiento cuántico muy bajo. Además, en experimentos de fotólisis continua en escala preparativa no se ha podido verificar la formación de fotoproductos en cantidades detectables, descartando cualquier otro proceso fotoquímico posible. 
CAPITULO 7

PROPIEDADES FOTOQUIMICAS Y FOTOFISICAS DE

$\left[\right.$ fac-(dppz) $\operatorname{Re}^{I}(\mathrm{CO})_{3}(4,4$ 'bipiridina $\left.)\right]\left(\mathrm{O}_{3} \mathrm{SCF}_{3}\right)$ 


\section{Resultados experimentales y Discusión}

\section{Materiales}

El complejo (dppz) $\operatorname{Re}^{\mathrm{I}}(\mathrm{CO})_{3}\left(4,4^{\prime} \text { 'bipi }\right)^{+}$fue preparado como se describe en el capítulo 5 . Las especificaciones de los solventes, aminas y otros reactivos utilizados de uso común, ya fueron señaladas en capítulos anteriores.

\section{Equipos y métodos}

Los espectros de luminiscencia del complejo (dppz) $\operatorname{Re}^{\mathrm{I}}(\mathrm{CO})_{3}\left(4,4^{\prime} \text { bipi }\right)^{+}$en estado estacionario fueron tomados de manera similar a las descriptas en los capítulos 1 y 3 así como la determinación de los rendimientos cuánticos de emisión, experimentos de láser flash fotólisis, espectrolectroquímica, etc. Los espectros de emisión a 77 K se tomaron de soluciones del complejo, transformadas en una matriz rígida de vidrio, en EtOH/MeOH (4:1), utilizando celdas de geometría cilíndrica. Las celdas fueron colocadas en el accesorio de baja temperatura del espectrofluorómetro Perkin-Elmer LS 50B. Los espectros UV-visible fueron tomados en un espectrofotómetro de doble haz Cary 3 utilizando celdas de cuarzo de $1 \mathrm{~cm}$ de camino óptico. Para algunos experimentos de láser flash fotólisis se utilizó un láser de nitrógeno Laseroptics con pulsos de luz de 337 nm y 10 ns de duración.

Otros detalles se mencionarán convenientemente en cada caso. 


\section{Espectros de absorción y de emisión UV-visible}

La naturaleza de las diferentes bandas presentes en el espectro de absorción UV-visible del complejo (dppz) $\operatorname{Re}^{\mathrm{I}}(\mathrm{CO})_{3}\left(4,4^{\prime} \text { bipi }\right)^{+}$en distintos solventes fue analizada anteriormente (ver capítulo 5). Básicamente, posee un máximo de absorción a $\lambda_{\max } \approx 280 \mathrm{~nm}$, transición intraligando, y absorciones en la zona comprendida entre 350 y $500 \mathrm{~nm}$ correspondientes a la absorción solapada de transiciones $\pi \rightarrow \pi^{*}$ centradas en el ligando dppz, IL, con una transición correspondiente a una transferencia de carga d $\pi\left(\operatorname{Re}^{\mathrm{I}}\right) \rightarrow \pi *(\mathrm{dppz}), \mathrm{TCML}_{\mathrm{Re} \rightarrow \mathrm{dppz}}$. La presencia de esta banda de absorción se observa claramente en el espectro diferencia entre los espectros de absorción del complejo con dppz y el ligando dppz libre, $\lambda_{\max } \approx 420 \mathrm{~nm}$. En la figura 1 se muestran, para su comparación, los espectros de absorción de estos compuestos y en el insertado el espectro diferencia. El solapamiento entre las bandas de absorción del estado IL y del TCML $L_{\mathrm{Re} \rightarrow \text { dppz }}$ es evidente y es un punto clave en el análisis de los estados excitados puestos en juego luego de la excitación con luz de $\lambda$ adecuada.

Los espectros de luminiscencia fueron tomados utilizando soluciones deaereadas con $\mathrm{N}_{2}$ del complejo $(d p p z) \operatorname{Re}^{\mathrm{I}}(\mathrm{CO})_{3}\left(4,4^{\prime} \text { 'bipi }\right)^{+}$a temperatura ambiente, y excitando a $\lambda=350,380,400$ y $420 \mathrm{~nm}$ en $\mathrm{CH}_{3} \mathrm{CN}$ y $\mathrm{CH}_{2} \mathrm{Cl}_{2}$ Los espectros corregidos, mostrados en la figura 2, presentan máximos de emisión en la zona comprendida entre 550 y 610 nm que se desplazan ligeramente cuando cambia la polaridad del solvente. La longitud de onda de irradiación no tiene un efecto significativo sobre la forma del espectro. Sin embargo, en DCM, la intensidad del máximo local en $\lambda \sim 600 \mathrm{~nm}$ se incrementa cuando crece el $\lambda_{\text {exc }}$. Los espectros presentan rasgos de emisión estructurada típica de estados tripletes intraligando, emisión ${ }^{3}\left(\pi^{*} \rightarrow \pi\right)$.

La forma de los espectros tomados a temperatura ambiente no difieren significativamente de los espectros de emisión tomados a 77 K, condiciones bajo las cuales la emisión estructurada de tipo $^{3}\left(\pi^{*} \rightarrow \pi\right)$ se observa, normalmente, en forma más acentuada, figura 3 . 


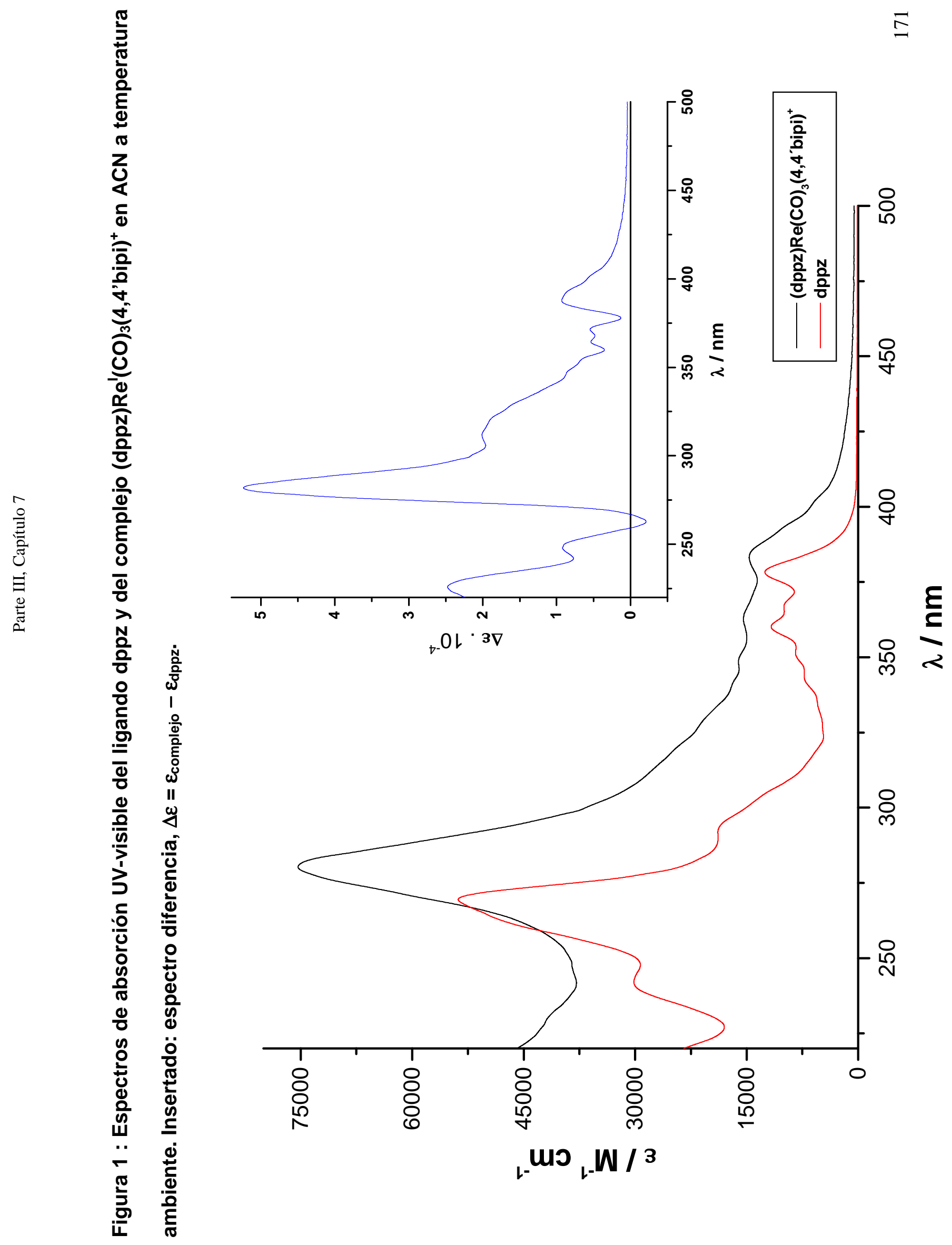


Figura 2: Espectros de emisión corregidos tomados a temperatura ambiente del complejo (dppz) $\operatorname{Re}^{\prime}(\mathrm{CO})_{3}\left(4,4^{\prime} \text { 'bipi }\right)^{+}$en los solventes, a) $\mathrm{ACN}$ y b) DCM

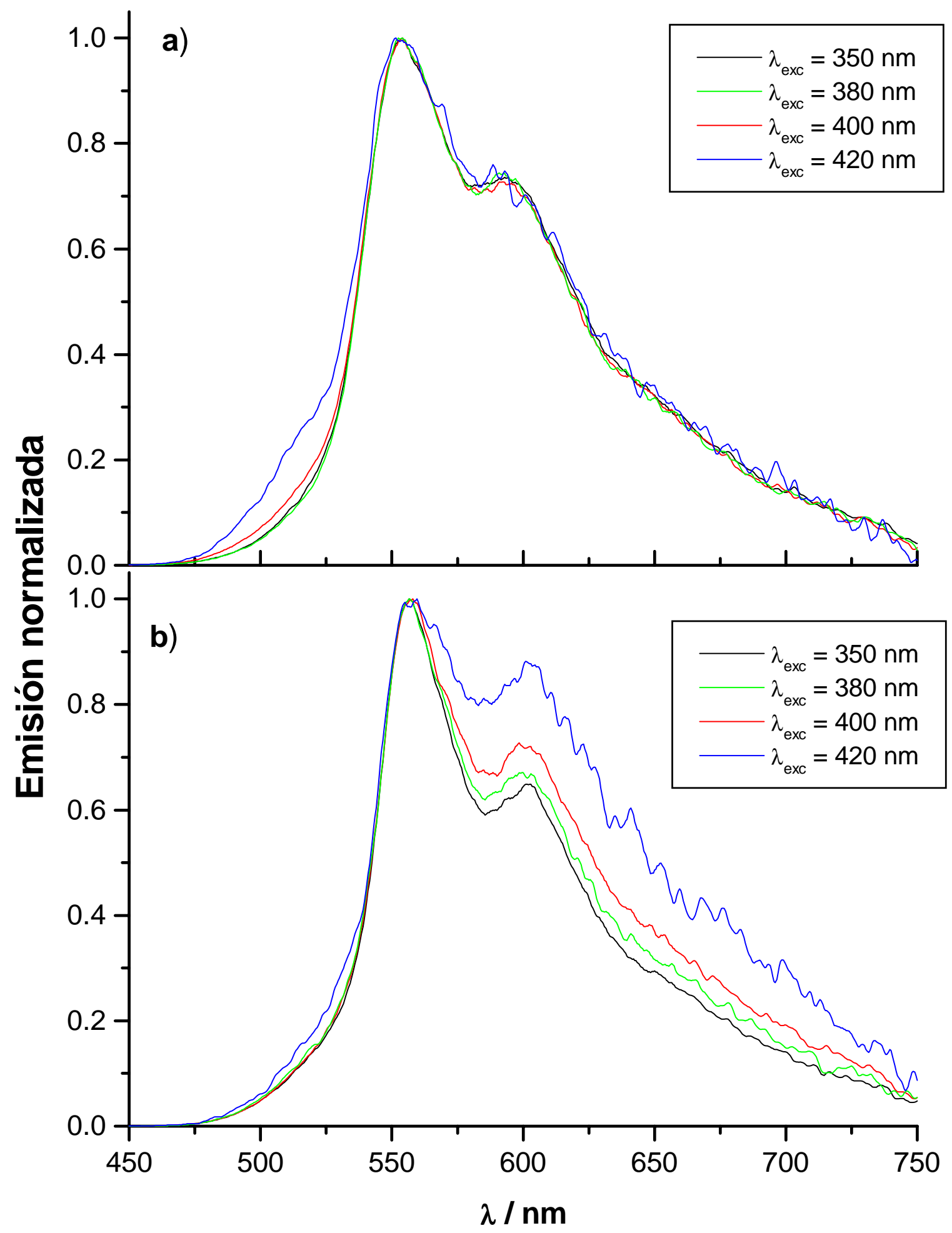


Estos resultados sugieren que la emisión predominante en el complejo de $\operatorname{Re}^{\mathrm{I}}$ proviene de un estado triplete intraligando del dppz, ${ }^{3} \mathrm{IL}$.

Tal asignación de la luminiscencia observada, coincide con resultados similares obtenidos para sistemas relacionados $[4,5,6,15]$. Además, los rasgos de la emisión en la zona de los 600 $\mathrm{nm}$ podrían corresponder a una emisión proveniente de un estado excitado ${ }^{3} \mathrm{TCML}_{\mathrm{Re} \rightarrow \mathrm{dppz}}$ solapada con la emisión ${ }^{3} \mathrm{IL}$ del dppz. Este estado de transferencia de carga puede ser poblado con mayor eficiencia excitando a $\lambda \geq 400 \mathrm{~nm}$ debido a que en esta región se encuentra la banda de absorción correspondiente a la transición estado fundamental $\rightarrow{ }^{1} \mathrm{TCML} L_{\mathrm{Re} \rightarrow \mathrm{dppz}}$

Figura 3: Espectros de emisión corregidos tomados a $77 \mathrm{~K}$ del complejo (dppz)Re'(CO) $)_{3}\left(4,4^{\prime}\right.$ 'bipi) ${ }^{+}$en EtOH/MeOH (4:1)

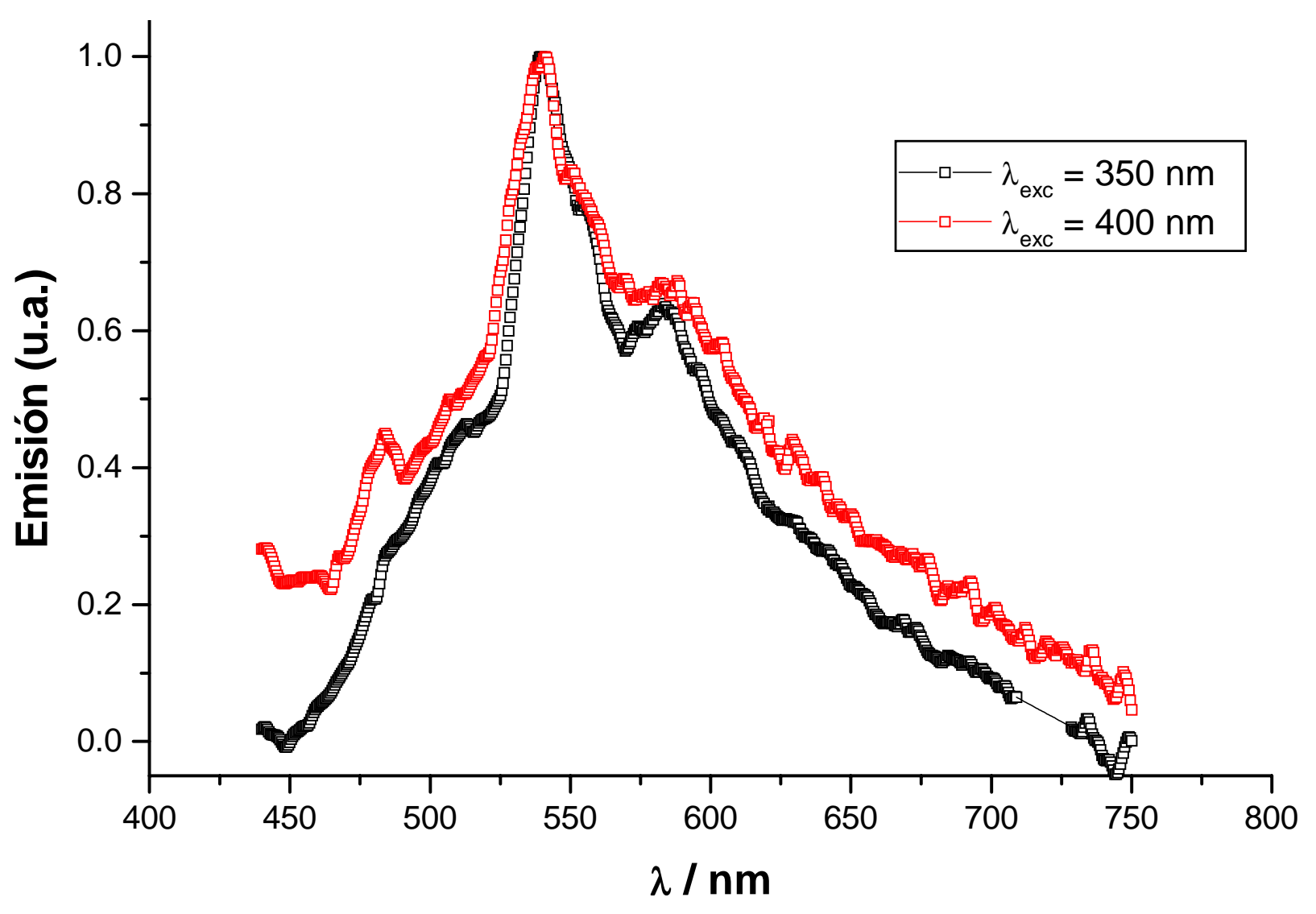


Por otro lado, el espectro de excitación, corregido por absorción, y los rendimientos cuánticos de emisión muestran evidencia adicional para esta apreciación. El máximo de excitación encontrado excitando a $\lambda \geq 400 \mathrm{~nm}$, figura 4 , es consistente con los $\phi_{\mathrm{emi}}$ calculados excitando a 350,400 y $436 \mathrm{~nm}$ en $\mathrm{CH}_{3} \mathrm{CN}$ y $\mathrm{CH}_{2} \mathrm{Cl}_{2}$ mostrados en la tabla I.

Figura 4: Espectro de excitación corregido obtenido con una solución deaereada del complejo (dppz) $\operatorname{Re}^{\prime}(\mathrm{CO})_{3}\left(4,4^{\prime} \mathrm{bipi}^{+}\right.$en acetonitrilo. $\lambda_{\text {obs }}=550 \mathrm{~nm}$

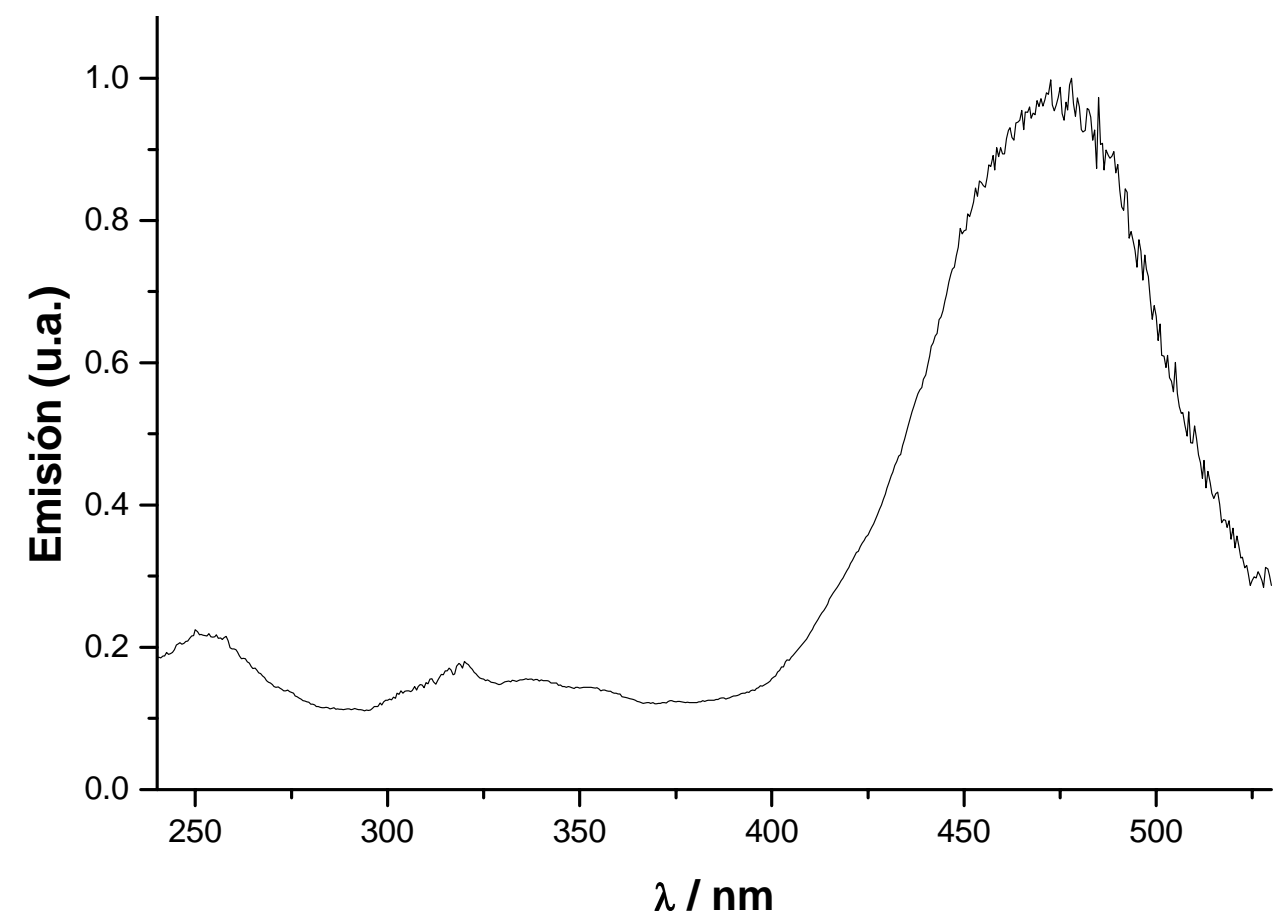

Tabla I: Rendimientos cuánticos de emisión, en acetonitrilo y diclorometano, del complejo (dppz)Re'(CO) $3\left(4,4^{\prime}\right.$ bipi) ${ }^{+}$excitando con $\lambda=350,400$ y $436 \mathrm{~nm}$

\begin{tabular}{cccc}
\hline Solvente & ${ }^{\mathrm{a}} \boldsymbol{\phi}^{350}$ & ${ }^{\mathrm{a}} \boldsymbol{\phi}^{400}$ & ${ }^{\mathrm{b}} \boldsymbol{\phi}^{\mathbf{4 3 6}}$ \\
\hline $\mathrm{CH}_{2} \mathrm{Cl}_{2}$ & $(1,4 \pm 0,6) \times 10^{-3}$ & $(1,6 \pm 0,4) \times 10^{-3}$ & $8,5 \times 10^{-3}$ \\
$\mathrm{CH}_{3} \mathrm{CN}$ & $(1,3 \pm 0,2) \times 10^{-3}$ & $(1,5 \pm 0,3) \times 10^{-3}$ & $7,8 \times 10^{-3}$ \\
\hline
\end{tabular}

Calculados a $\mathrm{T}_{\mathrm{amb}}$. usando como referencia: a. Rodamina B en EtOH $\phi=0,69$. b. Ru(bipi) ${ }_{3}^{+2}$ en ACN $\phi=0,062$ 


\section{Estudio del quenching de la emisión por agua}

Con el objetivo de sumar información para dilucidar cuáles son los estados excitados involucrados en el complejo de $\mathrm{Re}^{\mathrm{I}}$ en estudio, se realizaron experimentos de quenching de la emisión en estado estacionario usando $\mathrm{H}_{2} \mathrm{O}$ como quencher. Previamente, se había observado que el complejo en medio acuoso no presentaba emisión significativa.

En complejos similares de $\mathrm{Ru}^{\mathrm{II}}$, Os ${ }^{\mathrm{II}}, \mathrm{Rh}^{\mathrm{III}}$, etc, que poseen el ligando dppz, se ha establecido que el estado excitado emisor de más baja energía es del tipo ${ }^{3} \mathrm{TCML}_{\mathrm{M} \rightarrow \mathrm{dppz}}$. Este estado excitado es fuertemente desactivado en contacto con el $\mathrm{H}_{2} \mathrm{O}$ por un mecanismo de puentes de hidrógeno del estado excitado y/ó transferencia de protones hacia los nitrógenos de la porción fenacínica del dppz [1,8-9,14,17,24].

En los complejos de $\operatorname{Re}^{\mathrm{I}}$ que poseen el ligando dppz la naturaleza del estado excitado de menor energía no está clara todavía, aunque algunos trabajos postulan que es del tipo ${ }^{3} \mathrm{IL}$ del dppz [5,15,23]. Estudios espectroscópicos realizados sobre soluciones del ligando dppz libre en acetonitrilo en presencia y en ausencia de agua no mostraron grandes diferencias en sus propiedades fotofísicas. Por lo tanto, el estado IL, en principio, no se vería afectado directamente por la presencia de $\mathrm{H}_{2} \mathrm{O}$, sin embargo, en el complejo de $\mathrm{Re}^{\mathrm{I}}$ en estudio, los experimentos de emisión en estado estacionario llevados a cabo en soluciones en acetonitrilo deaereadas del $(\mathrm{dppz}) \operatorname{Re}^{\mathrm{I}}(\mathrm{CO})_{3}\left(4,4^{\prime} \text { bipi }\right)^{+}$con cantidades crecientes de agua muestran que el quenching acuoso de la emisión se verifica, figura 4a. Esto ha sido racionalizado en un complejo similar asumiendo que en medio acuoso los dos estados están lo suficientemente próximos en energía como para proveer un camino adicional para la desactivación del estado ${ }^{3} \mathrm{IL}$ vía un proceso de conversión interna [5]. El quenching de la emisión, en este tipo de complejos, es consecuencia de la rápida desactivación ejercida por el agua sobre el estado

${ }^{3}$ TCML desplazando el sistema hacia una condición de despoblamiento del estado ${ }^{3} \mathrm{IL}$ 
produciendo, en definitiva, el quenching indirecto de la emisión predominante del complejo de $\operatorname{Re}^{\mathrm{I}}$ :

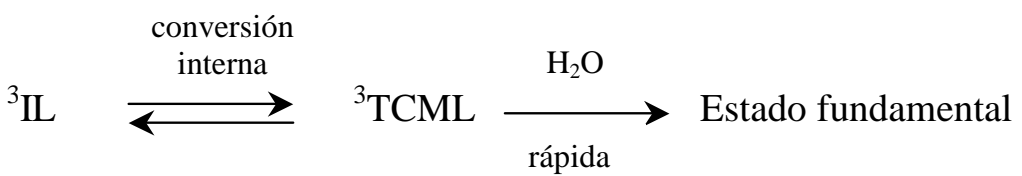

Los gráficos de Stern-Volmer construidos con los datos experimentales obtenidos para el complejo (dppz) $\operatorname{Re}^{\mathrm{I}}(\mathrm{CO})_{3}\left(4,4^{\prime} \text { bipi }\right)^{+}, \phi^{\mathrm{o}} \phi$ vs $\left[\mathrm{H}_{2} \mathrm{O}\right]$, (donde $\phi^{\mathrm{o}}$ y $\phi$ corresponden a los rendimientos cuánticos de emisión en ausencia y en presencia de $\mathrm{H}_{2} \mathrm{O}$, respectivamente) presentan un comportamiento atípico, siendo no lineales y con curvatura hacia arriba, figura 4b. Además, el quenching se verifica en forma apreciable cuando la $\left[\mathrm{H}_{2} \mathrm{O}\right]$ es suficientemente alta sugiriendo un mecanismo de desactivación en donde la solvatación debe ser total. Los tiempos de vida de la emisión obtenidos para cada solución no parecen ser afectados, dentro del error experimental. Esto implica que el mecanismo de quenching no es solamente difusional y que existe una contribución importante al proceso del quenching estático. Estos resultados son consistentes con el modelo de quenching denominado de esfera de Perrin [21]. Este mecanismo asume que las moléculas emisoras son desactivadas solo si la molécula quencher se encuentra dentro de un volumen esférico, $V$, de radio $r$ alrededor de la misma. De esta manera, las moléculas de $\mathrm{H}_{2} \mathrm{O}$ que estén a una distancia $d>r$ de una molécula emisora no podrán desactivarla.

La ecuación para el tratamiento de los datos experimentales, acorde con el modelo de esfera de Perrin es la siguiente:

$$
\ln \left(\phi^{\circ} / \phi\right)=V \mathrm{~N}\left[\mathrm{H}_{2} \mathrm{O}\right]
$$


Figura 4: a) Espectros de emisión en estado estacionario de soluciones deaereadas del complejo (dppz)Re'(CO) ${ }_{3}\left(4,4^{\prime} \text { bipi }\right)^{+}$en $A C N$ con cantidades crecientes de $\mathrm{H}_{2} \mathrm{O}, \lambda_{\mathrm{exc}}=350 \mathrm{~nm}$. b) Gráficos de Stern - Volmer.
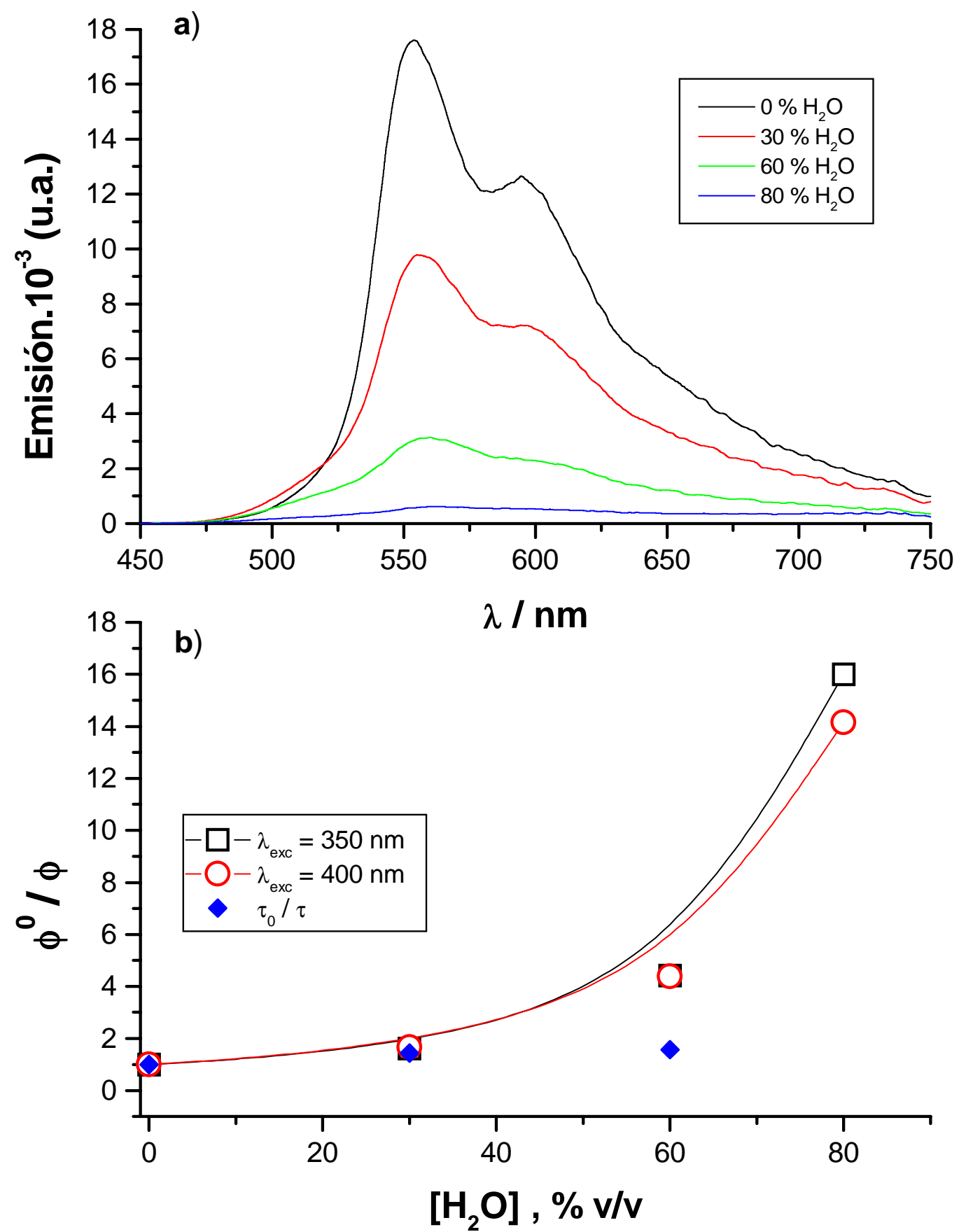
donde $\phi$ y $\phi^{\mathrm{o}}$ son, respectivamente, los rendimientos cuánticos de emisión con y $\sin \mathrm{H}_{2} \mathrm{O}, V$ es el volumen de la esfera y $\mathrm{N}$ es el número de Avogadro.

De esta expresión se puede inferir que una gráfica de $\ln \left(\phi^{\circ} / \phi\right)$ vs $\left[\mathrm{H}_{2} \mathrm{O}\right]$ debe ser una recta y de la pendiente se puede obtener el valor de $V$.

Los valores experimentales obtenidos para el quenching de la emisión del complejo $(\mathrm{dppz}) \mathrm{Re}^{\mathrm{I}}(\mathrm{CO})_{3}\left(4,4^{\prime} \text { bipi }\right)^{+}$por $\left[\mathrm{H}_{2} \mathrm{O}\right]$ pudieron ajustarse mejor usando esta expresión, figura 5. Asumiendo que el volumen de la esfera $V=4 / 3 \pi r^{3}$ se obtuvo un valor de $r=2,9 \AA$.

El modelo de esfera de Perrin ha sido propuesto para el quenching acuoso de la emisión del complejo pariente $\mathrm{Ru}(\mathrm{phen})_{2}(\mathrm{dppz})^{+2}$ [25], por lo tanto asumiremos que el complejo de $\mathrm{Re}^{\mathrm{I}}$ en estudio es un nuevo sistema que verifica este atípico mecanismo.

Figura 5: Gráfico correspondiente al ajuste de los datos experimentales usando el modelo de esfera de Perrin para la desactivación de la emisión del complejo (dppz) $\operatorname{Re}^{\prime}(\mathrm{CO})_{3}\left(4,4^{\prime} \text { bipi }\right)^{+}$por agua

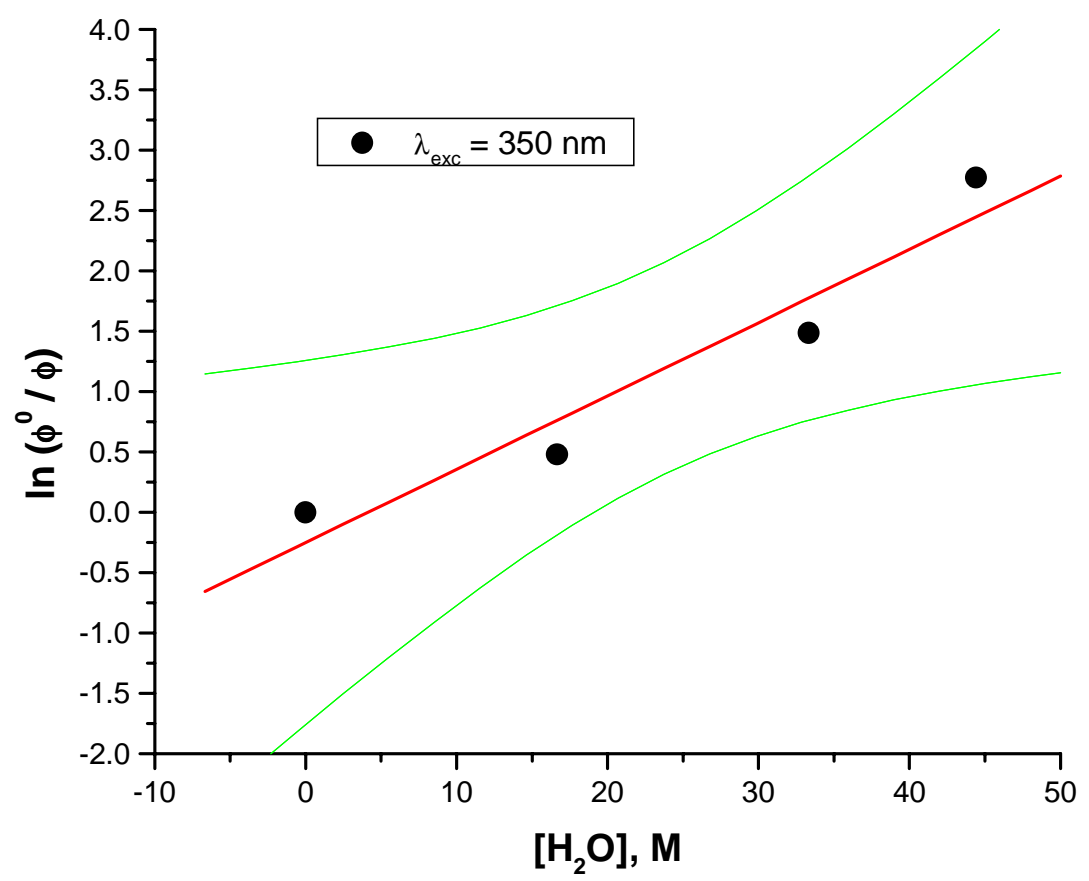




\section{Experimentos de láser flash fotólisis}

\section{i. $\quad$ Estudio de las especies transitorias generadas en distintos solventes}

Con el objeto de caracterizar las especies transitorias generadas luego de la excitación láser, se llevaron a cabo experimentos de flash fotólisis utilizando un láser de excímero de $\mathrm{XeF}, \lambda_{\mathrm{exc}}$ $=351 \mathrm{~nm}$, en soluciones deaereadas con $\mathrm{N}_{2}$, del complejo de $\mathrm{Re}^{\mathrm{I}}$ en estudio del orden de $10^{-4}$ $10^{-5} \mathrm{M}$ en solventes de distinta polaridad. En la figura 6 se muestran los rasgos del espectro de absorción UV-visible de la especie transitoria obtenida de soluciones del complejo $(\mathrm{dppz}) \operatorname{Re}^{\mathrm{I}}(\mathrm{CO})_{3}\left(4,4^{\prime} \text { bipi }\right)^{+}$en acetonitrilo y diclorometano. Estos espectros son muy similares a los obtenidos para el ligando dppz en los mismos solventes (ver capítulo 6, figura 4). El máximo de absorción observado en $\lambda \approx 470 \mathrm{~nm}$ es un rasgo característico del estado excitado ${ }^{3}\left(\pi, \pi^{*}\right)$ del ligando dppz, y también fue observado en el espectro del estado triplete de la fenacina $[5,22]$. Por otro lado, en los espectros no se observa un pico de absorción en $\lambda \approx 390$ nm. Esta banda de absorción observada en el ligando dppz libre ha sido relacionada con el estado excitado ${ }^{3}\left(\mathrm{n}, \pi^{*}\right)$. La ausencia de ella cuando el ligando dppz está coordinado al metal se puede atribuir a la imposibilidad de los nitrógenos de la porción bipiridínica del dppz (involucrados en la unión) para participar en una transición del tipo $n \rightarrow \pi^{*}$. Sin embargo, otras transiciones $n \rightarrow \pi^{*}$ podrían ser posibles involucrando los nitrógenos de la porción fenacínica del ligando dppz, aunque no parecen jugar un rol importante al menos a estos niveles de energía de irradiación.

Los espectros obtenidos son también muy similares a los encontrados en la bibliografía para complejos de $\operatorname{Re}^{\mathrm{I}}$ parientes y asignados al estado intraligante ${ }^{3}\left(\pi, \pi^{*}\right)[5,6,15]$. Por lo tanto, el espectro de absorción observado para la especie transitoria generada en el complejo $(\mathrm{dppz}) \operatorname{Re}^{\mathrm{I}}(\mathrm{CO})_{3}\left(4,4^{\prime} \text { bipi }\right)^{+}$puede ser asignada al estado excitado ${ }^{3}\left(\pi, \pi^{*}\right)$. 
Figura 6: Espectros de absorción de la especie transitoria obtenida irradiando soluciones deaereadas del complejo $(\mathrm{dppz}) \operatorname{Re}^{\prime}(\mathrm{CO})_{3}\left(4,4^{\prime} \mathrm{bipi}^{+}\right.$con pulsos de luz de $351 \mathrm{~nm}$ : a) $5,1 \cdot 10^{-5} \mathrm{M}$ en $\mathrm{ACN}$ y b) $3,3.10^{-5} \mathrm{M}$ en DCM
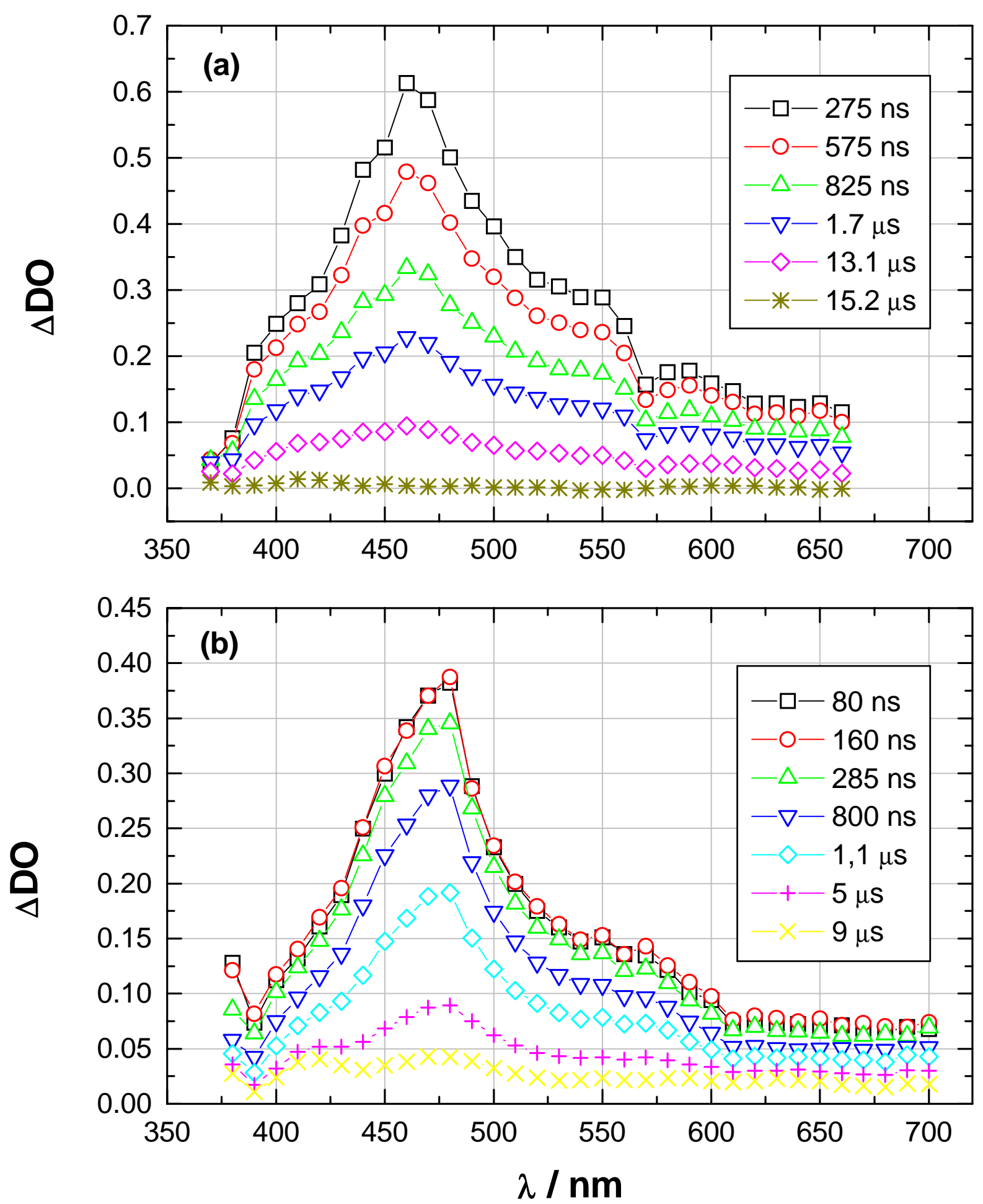
En los espectros de las especies transitorias obtenidas no se observan rasgos atribuibles a algún estado excitado de transferencia de carga, aún a escalas tiempos cortos luego del pulso del láser. Sin embargo no se descarta la formación del estado $\mathrm{TCML}_{\mathrm{Re} \rightarrow \mathrm{dppz}} \mathrm{y}$ posterior desaparición rápida, no detectable en nuestras condiciones de trabajo. Otra posibilidad es que coexista con el estado ${ }^{3}\left(\pi, \pi^{*}\right)$ desapareciendo ambos en forma paralela, con absorciones similares pero prevaleciendo el espectro de absorción del ${ }^{3}\left(\pi, \pi^{*}\right)$ por poseer coeficientes de extinción mayores a los del $\mathrm{TCML}_{\mathrm{Re} \rightarrow \mathrm{dppz}}$. Ambas opciones serán evaluadas a continuación analizando las cinéticas de desaparición de las especies transitorias en $\mathrm{ACN}, \mathrm{ACN} / \mathrm{H}_{2} \mathrm{O}$, DCM, MeOH y 2-PrOH junto con los espectros de absorción en estos solventes.

En acetonitrilo, la banda de absorción en $\lambda \approx 470 \mathrm{~nm}$ desaparece en forma monoexponencial. Sin embargo, los tiempos de vida varían con la longitud de onda siendo $\tau \sim 5 \mu$ s a $400 \mathrm{~nm}$ y $\tau$ $\sim 15 \mu$ s a $650 \mathrm{~nm}$, cuando las medidas se llevaron a cabo en una escala de tiempo de 10 $\mu \mathrm{s} /$ div. Del análisis de experimentos realizados a escalas de tiempo diferentes fue posible obtener dos exponenciales $\tau_{1}=5,2 \mu \mathrm{s}$ y $\tau_{2}=13,0 \mu \mathrm{s}$. La absorción residual que se mantiene a tiempos largos no presenta rasgos similares a los observados en la fotólisis del ligando dppz en las mismas condiciones experimentales. Por otro lado, en diclorometano, el decaimiento fue biexponencial con $\tau_{1}=0,230 \mu$ s y $\tau_{2}=3,1 \mu$ s cuando la escala de tiempo del experimento fue de $2 \mu \mathrm{s} /$ div. A escala de tiempos más largos (10 $\mu$ s/div) la desaparición de la especie transitoria fue de tipo monoexponencial con $\tau=8,6 \mu$ s y los espectros a distintos tiempos mostrados en la figura $6 \mathrm{~b}$ no difieren en forma apreciable. Sin embargo, en este caso, el producto residual muestra un máximo en $\lambda \approx 420 \mathrm{~nm}$ muy similar al observado en la fotólisis del dppz libre. Experimentos realizados en solventes donores de H como el metanol y el 2propanol acentúan la presencia de este producto final que perdura aún a tiempos mayores a los $100 \mu$ s luego del pulso del láser. En la figura 7a se muestra el espectro de la especie 
transitoria obtenido de soluciones en metanol del complejo de $\operatorname{Re}^{\mathrm{I}}$ en estudio, excitando a 355 nm donde se observa la desaparición gradual de la banda de absorción en $\lambda \approx 470 \mathrm{~nm}$ y la banda de absorción residual en $\lambda \approx 410 \mathrm{~nm}$. El espectro obtenido inicialmente en este solvente fue igual al obtenido trabajando a escala de tiempos más cortos, mostrando un decaimiento con tiempos de vida de 1 a $2 \mu \mathrm{s}$. Cuando experimentos similares se llevaron a cabo con soluciones $5.10^{-5} \mathrm{M}$ del complejo en $\mathrm{ACN} / \mathrm{H}_{2} \mathrm{O}(15 \mathrm{ml} / 35 \mathrm{ml})$ la especie transitoria formada posee rasgos de absorción similares a los observados en ACN y DCM, figura 7b. Sin embargo, los tiempos de vida son más cortos, $\tau \sim 1$ a $2,5 \mu \mathrm{s}$, comparables a los observados en $\mathrm{MeOH}$. Estos tiempos de vida más cortos pueden estar relacionados con el quenching de la luminiscencia ejercido por el agua, como vimos anteriormente, y probablemente signifique que la emisión proviene de las mismas especies. Si el mecanismo de quenching está basado en la formación de puentes de $\mathrm{H}$ entre las moléculas de agua y los nitrógenos de la porción fenacina del ligando dppz, el efecto producido en metanol podría explicarse de manera similar.

Los experimentos de luminiscencia flash resuelta en el tiempo se llevaron a cabo en soluciones, libres de $\mathrm{O}_{2}$, del complejo (dppz) $\operatorname{Re}^{\mathrm{I}}(\mathrm{CO})_{3}\left(4,4^{\prime} \text { bipi }\right)^{+}$en $\mathrm{ACN}$ y $\mathrm{DCM}$ a temperatura ambiente, en un equipo PTI $\left(\lambda_{\mathrm{exc}}=337 \mathrm{~nm}\right)$, en un láser de excímero $\left(\lambda_{\mathrm{exc}}=351\right.$ nm) y en un láser de $\mathrm{N}_{2}$ Laseroptics. Los tiempos de vida de la luminiscencia fueron complicados de determinar debido, tal vez, a los bajos rendimientos cuánticos de emisión. Sin embargo, se pudo obtener un valor de $\tau_{\text {emi }} \sim 2$ a $3 \mu$ s. Este valor del $\tau_{\text {emi }}$, es del orden de los tiempos de vida de las especies transitorias obtenidas en las medidas de absorción y asignadas al estado excitado ${ }^{3}\left(\pi, \pi^{*}\right)$, avalando esta asignación 
Figura 7: Espectros de absorción de la especie transitoria obtenida irradiando soluciones deaereadas del complejo $(\mathrm{dppz}) \mathrm{Re}^{\prime}(\mathrm{CO})_{3}\left(4,4^{\prime} \mathrm{bipi}^{+}\right.$: a) en $\mathrm{MeOH}, \lambda_{\text {exc }}$ $=355 \mathrm{~nm}$ y b) en $\mathrm{ACN} / \mathrm{H}_{2} \mathrm{O}, \lambda_{\text {exc }}=351 \mathrm{~nm}$
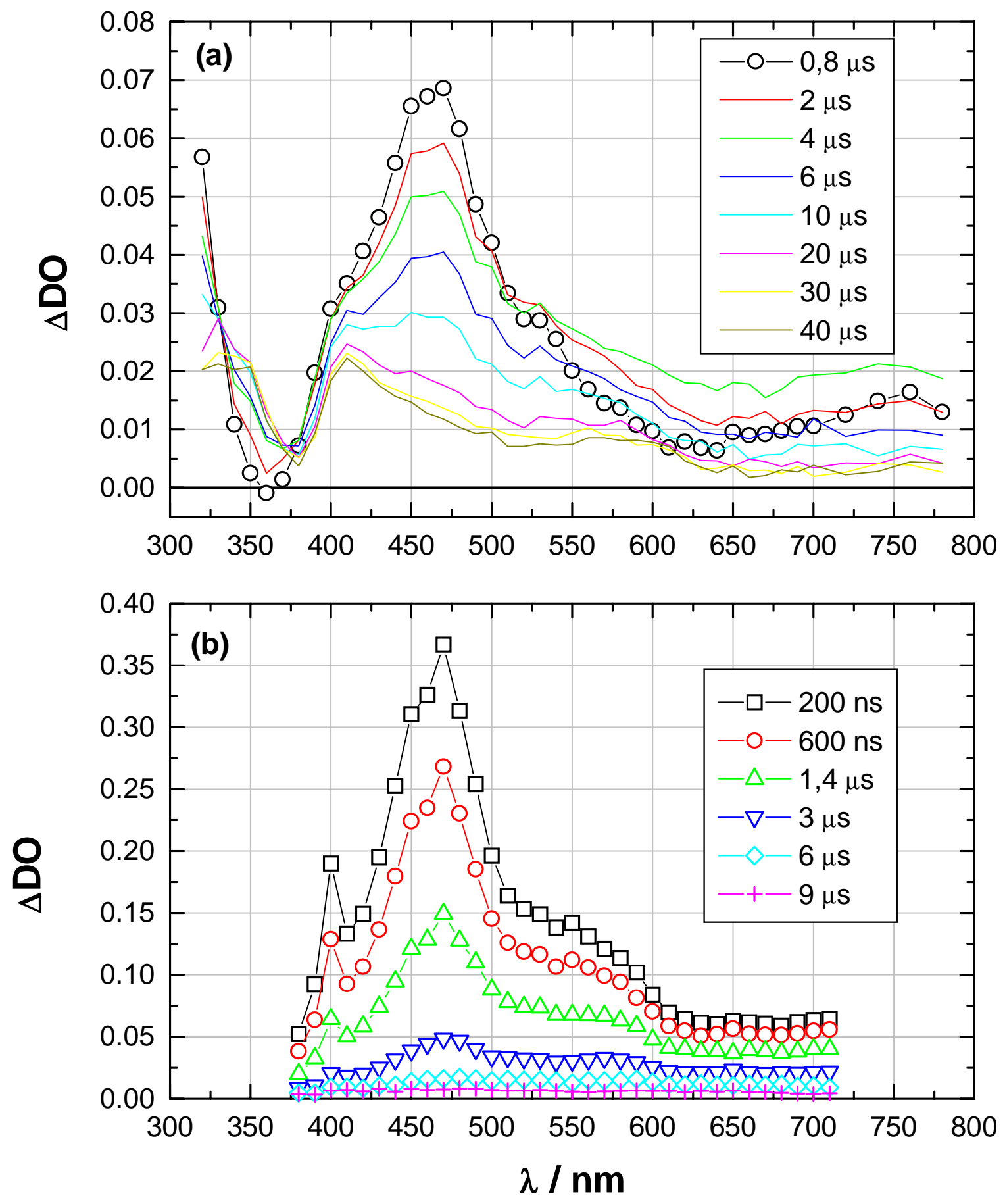


\section{ii. Quenching bimolecular del estado excitado}

Se llevaron a cabo experimentos de láser flash fotólisis, en condiciones similares a las anteriores, con soluciones del $(\mathrm{dppz}) \operatorname{Re}^{\mathrm{I}}(\mathrm{CO})_{3}\left(4,4^{\prime} \text { bipi }\right)^{+}$en presencia de trietilamina (TEA) $10^{-2} \mathrm{M}$ en $\mathrm{ACN}$. El espectro de absorción de la especie transitoria, figura 8, es muy diferente al obtenido en ausencia de TEA y muestra que uno ó más estados excitados han reaccionado con la amina. La amina donora de electrones, TEA, comúnmente es utilizada para reducir al estado excitado, generalmente de TCML y aceptor de electrones, para dar una especie radical de $\mathrm{Re}^{\bullet-}$, aunque, como vimos en el capítulo 6 , también reduce al estado ${ }^{3}\left(\pi, \pi^{*}\right)$ del dppz. El espectro observado no presenta una completa analogía con el obtenido, en condiciones similares, para el ligando dppz en presencia de TEOA (ver capítulo 6, figura 7a). Sin embargo, la absorción de $\lambda_{\max } \approx 420 \mathrm{~nm}$ podría, de todas maneras, corresponder a la especie reducida del dppz coordinado, similar a la generada en el ligando libre, fruto de la reacción entre el estado excitado ${ }^{3}\left(\pi, \pi^{*}\right)$ con la amina reductora. La otra banda ancha e intensa, ubicada entre 550 y $630 \mathrm{~nm}$, puede ser asignada al radical dppz ${ }^{\bullet-}$ generado, ahora, en la reacción de transferencia de electrones entre el estado excitado de transferencia de carga $\operatorname{TCML}_{\mathrm{Re} \rightarrow \mathrm{dppz}} \mathrm{y}$ la TEA. Aunque no teníamos evidencia espectroscópica de la presencia del estado TCML, este experimento, en donde se verifica la formación de dos radicales basados en el dppz, indicaría que ese estado excitado también está presente.

Las diferentes formas reducidas del ligando dppz, generadas por la reacción de los distintos estados excitados del complejo con TEA, podrían corresponder a especies en las cuales el electrón se encuentra localizado en orbitales de naturaleza diferente, energéticamente cercanos $[18,19]$. Como complemento a lo mencionado en los párrafos anteriores, existe también la posibilidad de que el $\mathrm{TCML}_{\mathrm{Re} \rightarrow 4,4 \text { bipi, }}$ pudiera estar presente y reaccionar con la amina para dar un radical donde el electrón estuviera ubicado en la 4,4’bipi. 
Figura 8: Espectros de absorción de la especie transitoria obtenida irradiando soluciones deaereadas del complejo $(\mathrm{dppz}) \operatorname{Re}^{\prime}(\mathrm{CO})_{3}\left(4,4^{\prime} \text { bipi }\right)^{+}$en presencia de TEA $10^{-2} \mathrm{M}$ en ACN. En el insertado: traza que muestra el decaimiento rápido de la DO hacia una situación de equilibrio entre las especies de vida larga

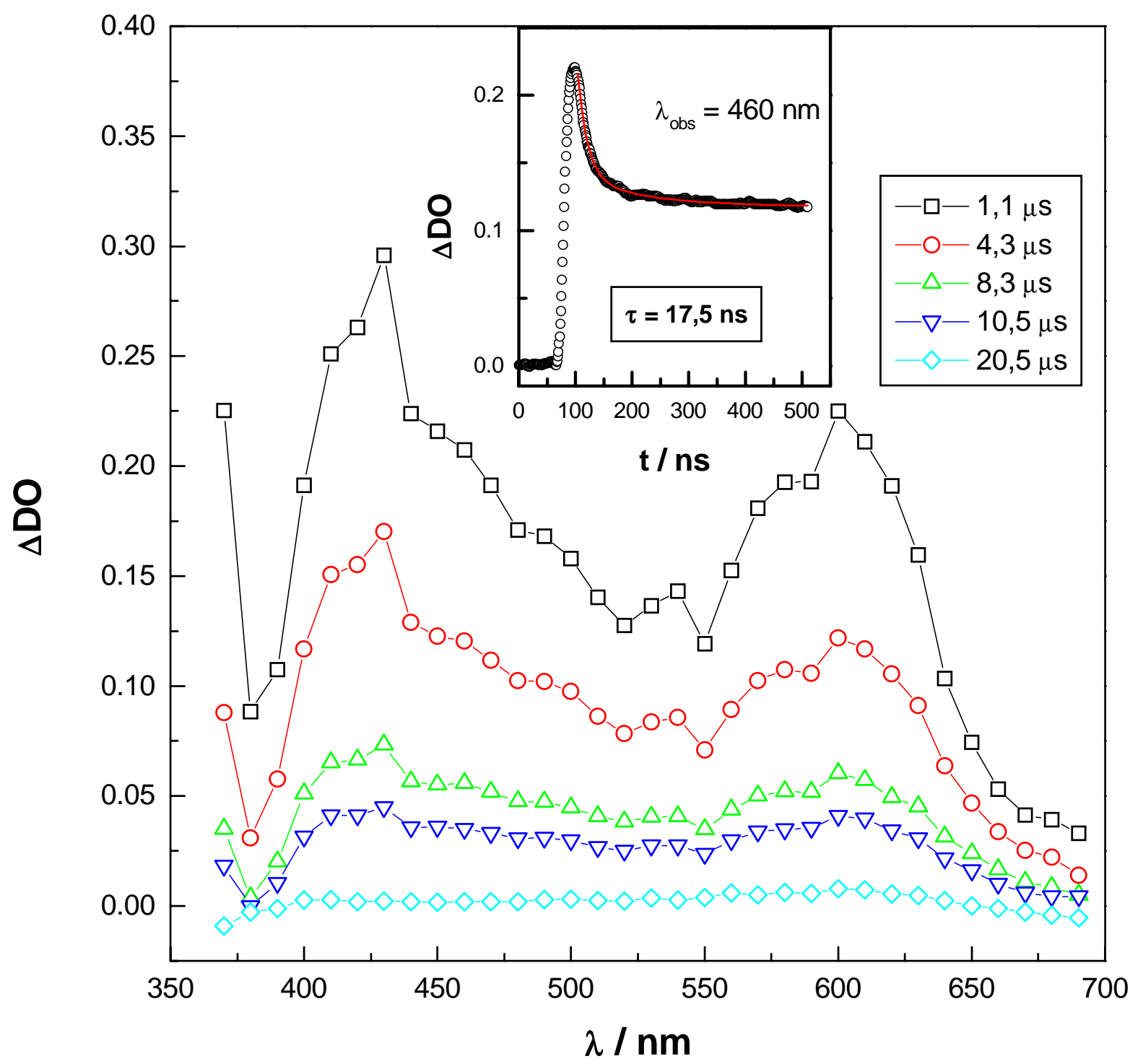

La espectroelectroquímica UV-visible de cada uno de los ligandos por separado aporta evidencia experimental que ayuda a evaluar las posibilidades expuestas. En la figura 9 se muestra el espectro de reflectancia relativa, $\Delta \mathrm{R} / \mathrm{R}$, del radical $\mathrm{dppz}^{\circ}$ obtenido en las 
condiciones descriptas en el capítulo 6, junto con el de la 4,4'bipi ${ }^{\bullet}$, obtenido en condiciones similares por modulación del potencial entre $-1,7$ y $-2,0 \mathrm{~V}$ vs $\mathrm{Ag} / \mathrm{AgCl} / \mathrm{KCl}_{\mathrm{sat}}$

El espectro de absorción del dppz ${ }^{\bullet-}$ electrogenerado presenta rasgos comparables con los que se observan en la figura 8. Este hecho avala lo propuesto anteriormente. Además, el ensanchamiento de la banda en $\lambda \approx 600 \mathrm{~nm}$ podría deberse a contribuciones a la absorción aportadas por el radical (dppz) $\operatorname{Re}^{\mathrm{I}}(\mathrm{CO})_{3}\left(4,4^{\prime} \mathrm{bipi}^{\bullet}\right)$ como sugiere el espectro de la figura 9a, aunque la presencia de esta especie no se puede confirmar.

Figura 9: Espectros obtenidos en experimentos de espectroelectroquímica con soluciones a) 4,5.10 $0^{-4} \mathrm{M}$ de 4,4'bipi y b) $3,3.10^{-4} \mathrm{M}$ de dppz, en ACN deaereado
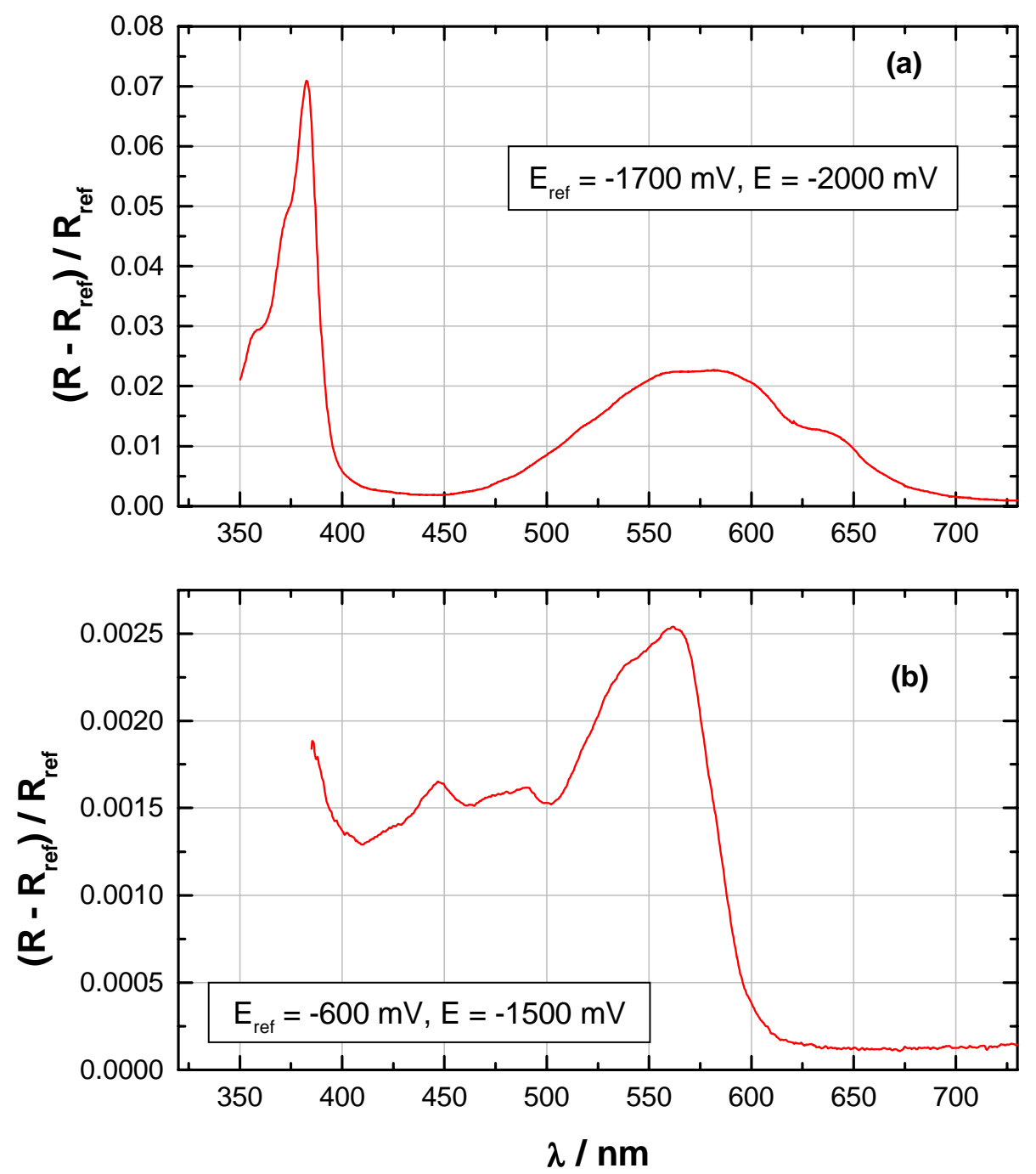
El espectro de las especies generadas por la reacción de la amina con los estados excitados del complejo decae en forma monoexponencial. Ello puede ser explicado considerando que las especies reducidas se encuentran en equilibrio, representadas como:

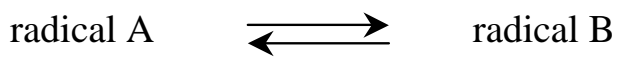

donde la situación más probable correspondería al caso en que los radicales A y B fueran las diferentes especies reducidas del ligando dppz en el complejo de $\mathrm{Re}^{\mathrm{I}}$ en estudio.

Además se puede considerar, como se observa en el insertado de la figura 8, que los radicales una vez formados relajan desde las concentraciones iniciales hasta alcanzar el equilibrio. Se puede, entonces, obtener la constante de velocidad específica $k=\tau^{-1}$ para el establecimiento del equilibrio, cuyo valor calculado fue $k=5,7 \cdot 10^{7} \mathrm{~s}^{-1}$. Del análisis cinético de las trazas obtenidas usando una escala de tiempo de $10 \mu \mathrm{s} /$ div se obtiene un $\tau=5,5 \mu$ s para la desaparición simultánea de las especies transitorias en equilibrio.

Por otro lado, se llevaron a cabo experimentos similares en soluciones $6.10^{-5} \mathrm{M}$ del complejo $(\mathrm{dppz}) \operatorname{Re}^{\mathrm{I}}(\mathrm{CO})_{3}\left(4,4^{\prime} \text { bipi }\right)^{+}$en presencia de metilviológeno $\left(\mathrm{MV}^{++}\right) 10^{-2} \mathrm{M}$, en acetonitrilo deaereado. El $\mathrm{MV}^{++}$es una mólecula de 4,4’bipiridina cuyos nitrógenos piridínicos se encuentran metilados. Es un compuesto muy utilizado en fotoquímica de complejos de metales de transición para caracterizar las propiedades redox de estados excitados, en particular, de los TCML. Como consecuencia de la reacción entre ambos, el $\mathrm{MV}^{++}$oxida al estado excitado TCML obteniéndose la especie reducida $\mathrm{MV}^{+}$. Esta última especie posee un espectro de absorción conocido y característico que puede ser monitoreado para verificar que la reacción de quenching bimolecular del estado excitado ha ocurrido.

En la figura 10 se muestra el espectro de absorción obtenido luego del pulso del láser de 351 nm. Los rasgos generales del espectro son similares a los observados en ausencia de $\mathrm{MV}^{++}$, aunque con leves diferencias. La más notable es la aparición de un pico bien definido e intenso a $390 \mathrm{~nm}$, que perdura en el tiempo. Posteriormente este pico se transforma en el 
máximo del espectro a medida que desaparece la absorción a $460 \mathrm{~nm}$, apareciendo, además, otra banda centrada en $610 \mathrm{~nm}$. El espectro final, que permanece hasta los $40 \mu \mathrm{s}$, posee los rasgos típicos del $\mathrm{MV}^{+}$: el máximo de absorción a $390 \mathrm{~nm}$ y una banda ancha centrada en 610 $\mathrm{nm}[26]$.

Figura 10: Espectros de absorción de la especie transitoria obtenida irradiando soluciones deaereadas del complejo $(\mathrm{dppz}) \mathrm{Re}^{\prime}(\mathrm{CO})_{3}\left(4,4^{\prime} \mathrm{bipi}^{+}\right.$en presencia de $\mathrm{MV}^{++} 10^{-2} \mathrm{M}$ en $\mathrm{ACN}$

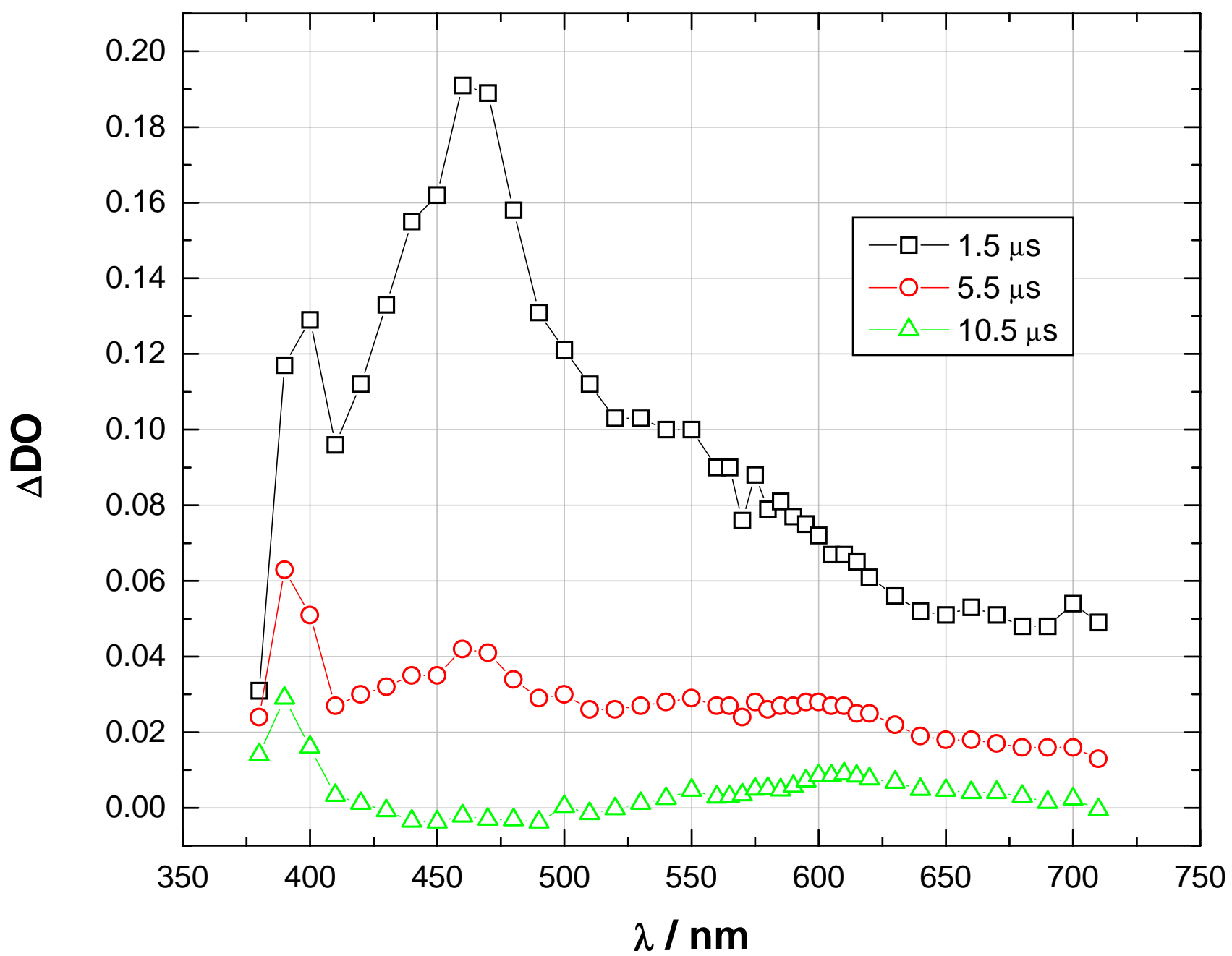


El espectro obtenido aporta evidencia concreta de que el estado excitado TCML participa en una reacción bimolecular de transferencia de electrones con este compuesto, en este caso como donor de electrones. Además, la presencia de los rasgos típicos del espectro de absorción del estado ${ }^{3}\left(\pi, \pi^{*}\right)$, solapados con el del $\mathrm{MV}^{+}$a tiempos cortos, sugiere que este estado no estaría involucrado en la reacción. La desaparición simultánea de ambos intermediarios es de tipo monoexponencial y los tiempos de vida dependen de la longitud de onda. En la zona de $420<\lambda<580 \mathrm{~nm}$ el $\tau=2,6 \mu$ s en $\lambda=390 \mathrm{~nm}$ el $\tau \sim 6,8 \mu$ s.

\section{Experimentos de fotólisis estacionaria}

Con el objetivo de caracterizar los posibles productos de reacción generados durante la fotólisis, se irradió con luz de $350 \mathrm{~nm}\left(\mathrm{I}_{0}=6,5.10^{-4}\right.$ Einstein $\left.1^{-1} \mathrm{~min}^{-1}\right)$ una solución del complejo (dppz) $\operatorname{Re}^{\mathrm{I}}(\mathrm{CO})_{3}\left(4,4^{\prime} \text { bipi }\right)^{+}$en $\mathrm{ACN}$ deaereado, en condiciones similares a las descriptas en el capítulo 6 para la fotólisis estacionaria del ligando dppz. Los pequeños cambios espectrales observados en el UV-visible luego de $1 \mathrm{~h}$ de fotólisis son análogos a los obtenidos para el ligando libre, figura 11a. De la misma manera, experimentos de fotólisis estacionaria a escala preparativa y fotolizando en forma continua durante $15 \mathrm{hs}$, no permitieron observar cambios apreciables en los espectros de absorción IR antes y después de la irradiación, figura 11b. Esto sugiere rendimientos cuánticos de reacción muy bajos de los intermediarios fotogenerados, regenerando finalmente el reactivo original. Adicionalmente se puede observar la ausencia de nuevas bandas de absorción, típicas de especies diméricas, en el espectro IR de la solución fotolisada. En experimentos similares llevados a cabo en otros complejos de $\operatorname{Re}^{\mathrm{I}}$ ha sido observado el crecimiento de bandas entre 1840 y $1880 \mathrm{~cm}^{-1}$ atribuidas a la formación de dímeros por recombinación de radicales de Re generados por pérdida de ligandos [27,28]. 
Figura 11: Fotólisis estacionaria irradiando con luz de $350 \mathrm{~nm}$ soluciones deaereadas del complejo $(\mathrm{dppz}) \operatorname{Re}^{\prime}(\mathrm{CO})_{3}\left(4,4^{\prime} \mathrm{bipi}^{+}\right.$en $\mathrm{ACN}$ antes y después de la irradiación. a) espectros UV-visible y b) espectros IR
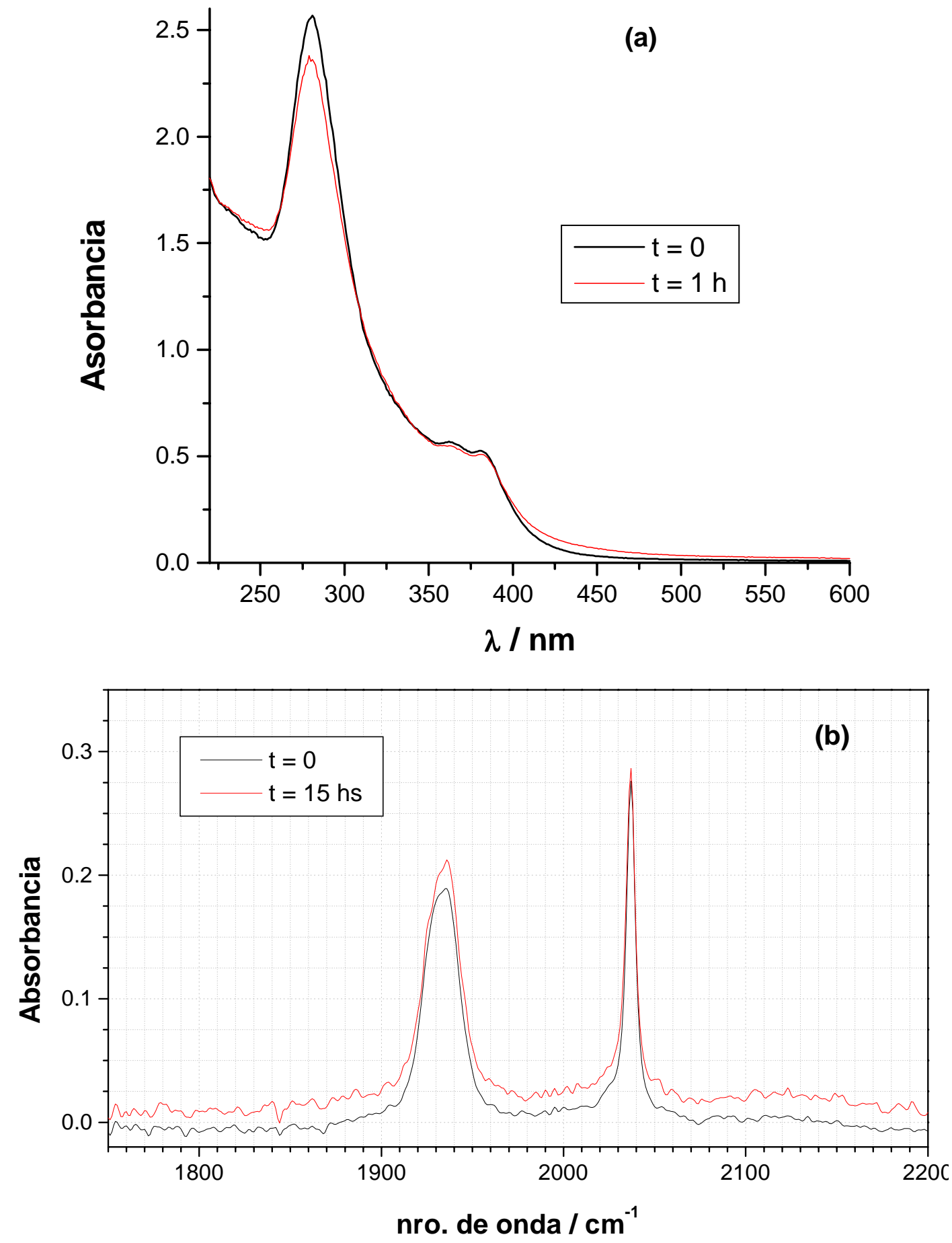
La aparición de otra banda $\sim 1820 \mathrm{~cm}^{-1}$ ha sido descripta, también, como característica de especies con puentes $\mathrm{CO}$ cuando soluciones del complejo $\mathrm{ClRe}(\mathrm{CO})_{3}$ (4-fenilpiridina) $)_{2}$ en ACN fueron fotolizadas [28]. En este caso los dímeros unidos por puentes CO son formados por pérdida de otras moléculas de carbonilo del complejo de Re'. En estos trabajos, se describe la desaparición simultánea de las bandas de absorción de los carbonilos presentes en la solución sin fotolizar. El espectro IR de las soluciones del $(\mathrm{dppz}) \operatorname{Re}^{\mathrm{I}}(\mathrm{CO})_{3}\left(4,4^{\prime} \text { bipi }\right)^{+}$antes y después de la fotólisis sugiere que el complejo de $\operatorname{Re}^{\mathrm{I}}$ en estudio presenta una alta fotoestabilidad.

\section{Conclusiones}

El análisis de los resultados permite ahora describir las propiedades fotoquímicas y fotofísicas del (dppz) $\operatorname{Re}^{\mathrm{I}}(\mathrm{CO})_{3}\left(4,4^{\prime} \text { bipi }\right)^{+}$en función de las propiedades de los estados excitados. Para ello, mencionaremos previamente, algunas generalizaciones que normalmente se tienen en cuenta, concernientes a la naturaleza de los estados excitados de más baja energía y a las propiedades de la luminiscencia en complejos del tipo $f a c$-(diimina) $\operatorname{Re}^{\mathrm{I}}(\mathrm{CO})_{3}(\mathrm{~L})^{+}$, donde diimina se refiere a un ligando piridínico bidentado como la 2,2’bipiridina ó 1,10-fenantrolina y L un ligando monodentado como la piridina y piridinas sustituidas, haluro, nitrilo ó isonitrilo [5]. La fotofísica de esta familia de compuestos ha sido intensamente estudiada y se ha determinado que, excepto en casos poco usuales, el estado excitado energéticamente más bajo corresponde a un ${ }^{3} \mathrm{TCML} \mathrm{Re}_{\mathrm{R} \rightarrow \text { dimina }}$ [2,3]. Estos estados excitados se caracterizan por poseer los siguientes rasgos: (1) la luminiscencia en solución a temperatura ambiente presenta una banda ancha, sin estructura, con un $\lambda_{\max }$ de emisión entre 500 y $650 \mathrm{~nm}$; (2) los rendimientos cuánticos de emisión son moderados $\left(0,01<\phi_{\mathrm{emi}}<0,3\right) ;(3)$ los tiempos de vida del estado excitado van desde 0,02 a $2 \mu \mathrm{s} ;$ y (4) la constante de velocidad para el decaimiento 
radiativo, $k_{\mathrm{r}}$, tiene un valor del orden de $10^{5}-10^{6} \mathrm{~s}^{-1}$ mientras que la constante de velocidad para el decaimiento no radiativo, $k_{\mathrm{nr}}$, del orden de $10^{6}-10^{7} \mathrm{~s}^{-1}$.

En los casos en que se ha determinado que el estado excitado de más baja energía correspondía a un estado centrado en el ligando diimina, $\left({ }^{3} \pi \pi^{*}\right)$, se pudo establecer que ese estado intraligando poseía una energía más baja que el estado excitado ${ }^{3} \mathrm{TCML}_{\mathrm{Re} \rightarrow \operatorname{dimina}}$ [29,30]. En estos casos, los rasgos característicos observados para la identificación del estado $\left({ }^{3} \pi \pi^{*}\right)$ fueron: (1) la luminiscencia en solución se observa con una estructura vibrónica bien resuelta; (2) la constante de velocidad radiativa es anormalmente baja; y (3) el espectro de absorción de la especie transitoria no posee los rasgos característicos del anión radical de la diimina.

Sobre la base de esta información y en función de los resultados obtenidos llegamos a la conclusión de que el estado excitado de más baja energía en el $(\mathrm{dppz}) \operatorname{Re}^{\mathrm{I}}(\mathrm{CO})_{3}\left(4,4^{\text {'bipi }}\right)^{+}$es un estado excitado del tipo $\left({ }^{3} \pi \pi^{*}\right)$ centrado en el ligando dppz. Esta asignación está basada en las siguientes observaciones: (1) la luminiscencia de las soluciones del complejo en solventes orgánicos tomados a temperatura ambiente se observa como una banda que posee estructura vibrónica resuelta. Los mismos rasgos de luminiscencia se observan a $77 \mathrm{~K}$, junto con una más acentuada estructuración de la emisión. El espaciamiento entre estos dos componentes vibrónicos de la emisión es similar al espaciamiento entre las dos bandas vibrónicas que se observan en los espectros de absorción del complejo en el UV-cercano asignadas a transiciones centradas en el ligando dppz; (2) el $\phi_{\mathrm{emi}}$ es $\sim 150$ veces más bajo que el valor promedio encontrado para esta familia de complejos y considerando un $\tau_{\mathrm{emi}}=3 \mu$ s en ACN y de 2,5 $\mu$ s en DCM las $k_{\mathrm{r}}$ toman un valor de $4,3.10^{2} \mathrm{~s}^{-1}$ y $5,6.10^{2} \mathrm{~s}^{-1}$, respectivamente, para cada uno de los solventes ${ }^{4)}$. Este valor bien bajo de $k_{\mathrm{r}}$ es similar a los observados en otros

\footnotetext{
4) Las constantes $k_{\mathrm{r}}$ y $k_{\mathrm{nr}}$ se calcularon teniendo en cuenta las siguientes consideraciones: $\tau_{\mathrm{emi}}=\left(k_{\mathrm{r}}+k_{\mathrm{nr}}\right)^{-1} \sim 1 / k_{\mathrm{nr}}$ $\mathrm{y} \phi_{\mathrm{emi}}=k_{\mathrm{r}} \tau_{\mathrm{emi}}$
} 
complejos de metales de transición $\mathrm{d}^{6}$ cuyas soluciones a temperatura ambiente presentan luminiscencia de estados ${ }^{3}$ IL [29,30]; y (3) los espectros de absorción de la especie transitoria obtenida en los experimentos de láser flash fotólisis del complejo son muy similares a los del ligando dppz libre, el cual ha sido asignado al estado excitado triplete del dppz.

La asignación del estado excitado de menor energía en el (dppz) $\operatorname{Re}^{\mathrm{I}}(\mathrm{CO})_{3}\left(4,4^{\prime} \text { bipi }\right)^{+}$como de naturaleza $\left({ }^{3} \pi \pi^{*}\right)$ intraligante ha sido también usada en los pocos ejemplos de esta nueva familia de complejos de $\operatorname{Re}^{\mathrm{I}}$ que poseen el ligando dppz [5-8,15,23]. Sin embargo, en nuestro caso, no descartamos la coexistencia del estado excitado de transferencia de carga ${ }^{3} \mathrm{TCML}_{\mathrm{Re} \rightarrow \mathrm{dpp} z}$ que se pone en evidencia en los experimentos de quenching bimolecular llevados a cabo en presencia de aminas reductoras como la TEA y compuestos reducibles como el $\mathrm{MV}^{+2}$. La energía de este estado ${ }^{3} \mathrm{TCML}_{\mathrm{Re} \rightarrow \mathrm{dppz}}$ estaría por encima y próxima a la del estado ${ }^{3} \mathrm{IL}$, pero no lo suficiente como para ser poblado térmicamente desde el estado $\left({ }^{3} \pi \pi^{*}\right)$ y proveer un paso más eficiente de desactivación radiativa. Esto es lo que ocurre en el caso del $\mathrm{ClRe}(\mathrm{CO})_{3}(\mathrm{dppz})$, donde la emisión observada a temperatura ambiente corresponde al estado excitado ${ }^{3} \mathrm{TCML}_{\mathrm{Re} \rightarrow \mathrm{dppz}}$ (emisión centrada en $600 \mathrm{~nm}$ ), mientras que el estado excitado de menor energía es el $\left({ }^{3} \pi \pi^{*}\right)$. La emisión estructurada correspondiente a este estado IL pudo ser caracterizada trabajando con soluciones del complejo a $77 \mathrm{~K}[15,23]$.

Por lo tanto, las propiedades fotofísicas del $(\mathrm{dppz}) \operatorname{Re}^{\mathrm{I}}(\mathrm{CO})_{3}\left(4,4^{\prime} \text { bipi }\right)^{+}$en solventes orgánicos pueden ser resumidas en un diagrama de Jablonski donde las energías relativas de los diferentes estados involucrados han sido estimadas de la siguiente forma: de los espectros de absorción se observa que al excitar el complejo con energías fotónicas $\mathrm{E}_{\mathrm{hv}} \leq 28,6.10^{3} \mathrm{~cm}^{-1}$ $\mathrm{mol}^{-1}\left(\lambda_{\text {exc }}=350 \mathrm{~nm}\right)$ se pueblan inicialmente estados excitados de carácter singletes del tipo transferencia de carga $\quad{ }^{1} \mathrm{TCML}, \quad{ }^{1}\left[\left(\mathrm{dppz}^{\bullet}\right) \operatorname{Re}^{\mathrm{II}}(\mathrm{CO})_{3}\left(4,4^{\prime} \text { bipi }\right)\right]^{+^{*}} \quad$ y $\quad\left({ }^{1} \pi \pi^{*}\right)$, $\left[\left({ }^{1} \mathrm{dppz}\right) \operatorname{Re}^{\mathrm{I}}(\mathrm{CO})_{3}\left(4,4^{\prime} \text { bipi }\right)\right]^{+^{*}}$ centrados en el ligando dppz, figura 12 . Cada uno de estos estados se desactivan por caminos no radiativos de conversión interna y de cruzamiento entre 
sistemas exclusivamente hacia el estado de energía más baja que es de naturaleza $\left({ }^{3} \pi \pi^{*}\right)$, $\left[{ }^{3} \mathrm{dppz}\right) \operatorname{Re}^{\mathrm{I}}(\mathrm{CO})_{3}\left(4,4^{\prime}\right.$ bipi $\left.)\right]^{+^{*}}$, el cual relaja hacia el estado fundamental por caminos radiativos y no radiativos. Basado en la banda de absorción de energía más baja, del espectro diferencia del insertado de la figura 1, y de los potenciales redox asignados en el complejo a las cuplas $\operatorname{Re}^{\mathrm{II}} / \mathrm{Re}^{\mathrm{I}}$, dppz / $\mathrm{dppz}^{\bullet-}$, pudo ser estimada la energía del estado ${ }^{1} \mathrm{TCML}, \mathrm{E}_{00}=2,7$ eV. La conversión desde este estado hacia el correspondiente estado triplete, ${ }^{3}$ TCML, ${ }^{3}\left[\left(\mathrm{dppz}^{\bullet-}\right) \operatorname{Re}^{\mathrm{II}}(\mathrm{CO})_{3}\left(4,4^{\prime} \text { bipi }\right)\right]^{+^{*}}$ es, presumiblemente, muy rápida debido al alto estado de mezcla singlete-triplete inducido por el acoplamiento spin-orbital del centro $\mathrm{d} \pi^{5}\left(\operatorname{Re}^{\mathrm{II}}\right)$, permitiendo acotar el valor de la constante de velocidad para este proceso en $k_{\text {isc }}>2.10^{8} \mathrm{~s}^{-1}$ [6]. La emisión está dominada, predominantemente, por la transición radiativa desde el estado $\left({ }^{3} \pi \pi^{*}\right)$ hacia el estado fundamental. Por lo tanto la energía de este estado excitado puede ser estimada de los espectros de emisión del complejo, $\mathrm{E}_{00}=2,4 \mathrm{eV}$.

En solventes orgánicos el estado excitado ${ }^{3}$ TCML tiene una energía más alta que el $\left({ }^{3} \pi \pi^{*}\right), \mathrm{E}_{00}$ $>2,4 \mathrm{eV}$, pero en un medio acuoso estos estados pueden estar, energéticamente, lo suficientemente cercanos como para permitir el establecimiento de un estado de equilibrio entre ambos [5]. La desactivación del estado de TCML ejercida por las moléculas de $\mathrm{H}_{2} \mathrm{O}$, en soluciones del complejo en $\mathrm{ACN}$, producen la desactivación indirecta del estado excitado luminiscente intraligando. El mecanismo de la desactivación ha sido satisfactoriamente ajustado al modelo de esfera de Perrin. El valor obtenido para el radio de la esfera, $r=2,9 \AA$, fue menor al encontrado para el complejo pariente $\mathrm{Ru}(\mathrm{phen})_{2}(\mathrm{dppz})^{+2}$ [25]. Este resultado correlaciona con las más altas $\left[\mathrm{H}_{2} \mathrm{O}\right]$ necesarias, en nuestros experimentos, para obtener un mismo grado de desactivación de la luminiscencia, y con la baja capacidad del ACN para generar enlaces por puentes de hidrógeno.

El efecto desactivador de la luminiscencia llevado a cabo por moléculas de agua podría ser aplicado, con el desarrollo de nuevos compuestos de esta familia de complejos de Re ${ }^{\mathrm{I}}$, como 
sensores de humedad ó como sondas luminiscentes de compartimentos hidrofóbicos en sistemas biológicos. Algunos de estos aspectos ya han sido desarrollados en compuestos similares de $\mathrm{Ru}^{\mathrm{II}}[31]$.

Figura 12: Diagrama de Jablonski que representa las energías relativas de los niveles electrónicos relevantes en la fotólisis del (dppz) $\operatorname{Re}^{\prime}(\mathrm{CO})_{3}\left(4,4{ }^{\prime} \text { bipi }\right)^{+}$ $(-)$ caminos radiativos $(-)$ caminos no radiativos

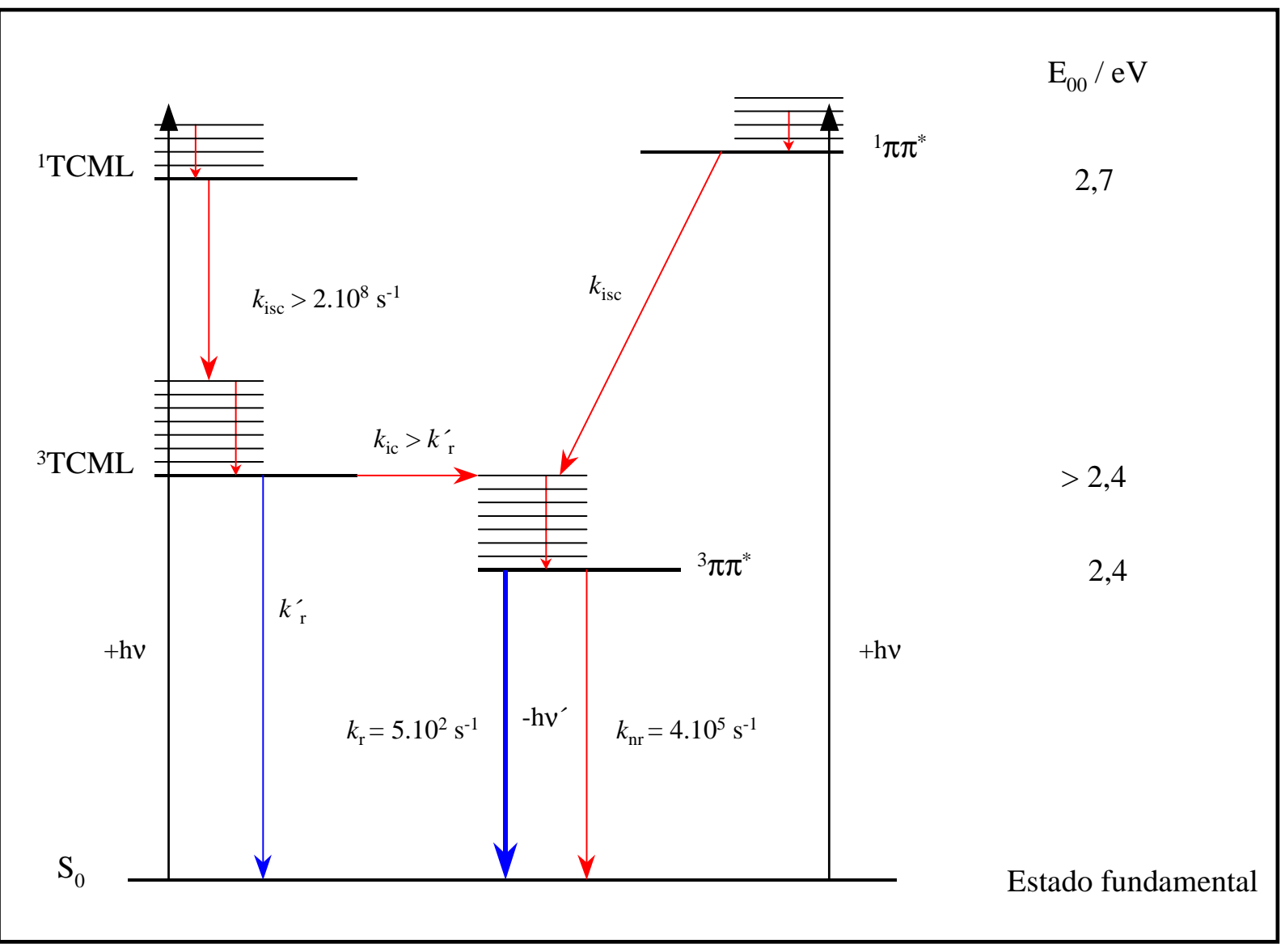


CAPITULO 8

\section{ESTUDIO DE INTERACCIÓN DEL $[$ fac-(dppz $) \operatorname{Re}^{\mathrm{I}}(\mathrm{CO})_{3}(4,4$ 'bipiridina $\left.)\right]\left(\mathrm{O}_{3} \mathrm{SCF}_{3}\right)$ CON POLINUCLEÓTIDOS SINTÉTICOS}




\section{Resultados experimentales y Discusión}

\section{Materiales y equipos utilizados}

Los espectros de absorción UV-visible se tomaron con un espectrofotómetro de doble haz Cary3 (Varian), usando celdas de cuarzo de $1 \mathrm{~cm}$ de camino óptico.

Los espectros de emisión se tomaron con un espectrofluorómetro Perkin-Elmer LS 50B a temperatura ambiente y se corrigieron por la diferente respuesta del instrumento y por la luz dispersada.

Se utilizaron soluciones de los polinucleótidos sintéticos Poly[dAdT]•Poly[dAdT] y Poly $[\mathrm{dGdC}] \bullet$ Poly $[\mathrm{dGdC}]\left(\right.$ Poly $[\mathrm{dAdT}]_{2}$ y Poly $\left[\mathrm{dGdC}_{2}\right)$, doble hélice de bases alternante, comprados a Pharmacia Biotech ${ }^{4)}$. Estos polinucleótidos son cadenas de ácido desoxiribonucleico (ADN) sintético cuya composición y secuencia de bases nitrogenadas corresponde, respectivamente, a ATATATAT...y GCGCGCGC... La otra cadena, necesaria para la formación de la doble hélice, es la correspondiente cadena complementaria. La unión de las cadenas se produce entre las bases nitrogenadas de cada una de ellas por formación de enlaces de hidrógeno. Estos puentes de $\mathrm{H}$ solamente se forman si en el enlace participa una base con su complementaria, es decir las uniones sólo se producen entre los pares de bases adecuados. Estos son adenina-timina y guanina-citosina que establecen una unión de forma específica.

Las soluciones se prepararon disolviendo cantidad suficiente del sólido liofilizado en solución fresca de un buffer acuoso TRIS $20 \mathrm{mM}-\mathrm{HCl}, 10 \%$ de $\mathrm{MeOH}$, ph $=7$ (TRIS =

\footnotetext{
${ }^{4)}$ Un polinucleótido es un polielectrolito compuesto por monómeros de desoxiribonucleótidos (mononucleótidos) unidos covalentemente. Cada monómero está constituido por un azúcar desoxiribosa unido a un grupo fosfato cargado negativamente y a una de las cuatro bases nitrogenadas posibles: adenina (A), guanina (G) (ambas bases purinas), citosina (C) y timina (T) (ambas bases pirimidinas) [1,32]
} 
Tris(hidroximetil)aminometano). El agua utilizada fue bidestilada en un equipo Milli- $Q$. En la figura 1 se muestran los espectros de absorción de una solución en buffer TRIS de cada uno de los polinucleótidos utilizados, donde no se observa, ó es despreciable, la absorción a $\lambda$ > $320 \mathrm{~nm}$. La concentración de los polinucleótidos en pares de base (pb) se determinó midiendo la absorción de la solución madre a $262 \mathrm{~nm}\left(\varepsilon=6,6.10^{3} \mathrm{M}^{-1} \mathrm{~cm}^{-1}\right)$ para el Poly[dAdT] 2 y a $254 \mathrm{~nm}\left(\varepsilon=8,4.10^{3} \mathrm{M}^{-1} \mathrm{~cm}^{-1}\right)$ para el Poly $[\mathrm{dGdC}]_{2}$ recalculando luego la concentración final en cada solución $[9,33,34]$.

Figura 1: Espectros de absorción de una solución en buffer TRIS 20 mM-HCl, $10 \%$ de $\mathrm{MeOH}, \mathrm{ph}=7$, de los polinucleótidos utilizados

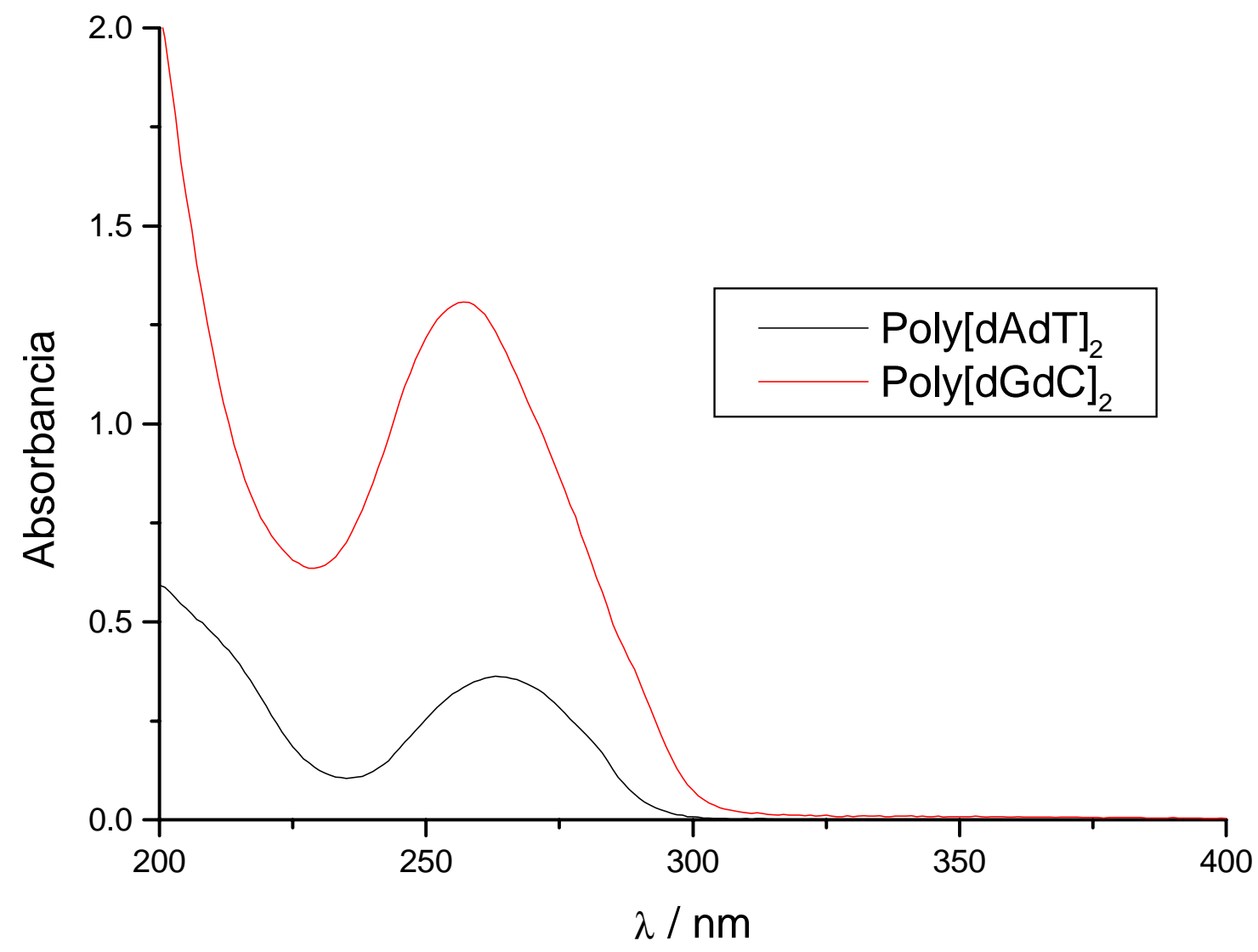


Las soluciones para los experimentos de interacción ('binding') fueron preparadas en forma tal que la concentración del complejo de $\mathrm{Re}^{\mathrm{I}}$ fuera siempre la misma e igual a 19,8 $\mu \mathrm{M}$, mientras que la concentración del polinucleótido en cada solución aumentaba (adicionando volúmenes crecientes de solución madre) de manera de obtener un aumento gradual, también, de la relación [polinucleótido] / [complejo de $\operatorname{Re}^{\mathrm{I}}$ ] (ó [Poly] / [Re]) para cada solución. El volumen final de cada solución fue de $1 \mathrm{ml}$.

Los espectros de emisión se obtuvieron usando celdas de cuarzo de cuatro ventanas de $1 \mathrm{~cm}$ de camino óptico x 0,4 cm de ancho con tapa de teflon y las soluciones fueron deaereadas con $\mathrm{N}_{2}$ durante 20 minutos antes de cada barrido.

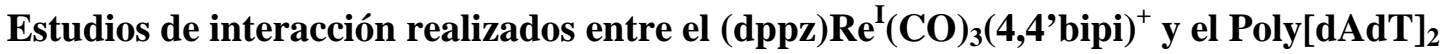

\section{i. $\quad$ Espectros de absorción $U V$-visible}

Con el objetivo de determinar la forma en que el complejo $(\mathrm{dppz}) \operatorname{Re}^{\mathrm{I}}(\mathrm{CO})_{3}\left(4,4^{\prime} \text { bipi }\right)^{+}$ interacciona con la doble hélice del Poly $[\mathrm{dAdT}]_{2}$, se prepararon soluciones del complejo y del polinucleótido con diferentes relaciones [Poly] / [Re] y se tomaron los espectros de absorción UV-visible para determinar cómo afecta, tal proceso, los rasgos del espectro.

Al aumentar en forma gradual la relación [Poly] / [Re] se observa una disminución en la absorbancia (hipocromismo) en la región de longitudes de onda comprendida entre 360 y 400 nm que comprende el conjunto de bandas vibrónicas asignadas al ligando dppz (ver capítulo 5). Estas bandas, además, sufren un pequeño corrimiento hacia el rojo (efecto batocrómico), hasta llegar a un valor en la relación [Poly] / [Re] a partir del cual estos dos efectos ya no se observan al seguir aumentando la concentración del polinucleótido. 
El hipocromismo y el corrimiento de la absorción hacia el rojo no fueron lo suficientemente importantes como para poder determinar una constante de interacción que caracterizara ese proceso por análisis de aquellos resultados.

Sin embargo, el efecto neto encontrado de hipocromismo y corrimiento batocrómico al comparar los espectros de las soluciones en presencia y ausencia de ADN sugiere algunos aspectos importantes de la interacción, figura 2.

En la literatura se pueden encontrar las diferentes formas observadas de interacción entre complejos de metales de transición con ligandos polipiridínicos y ácidos nucleicos [1,35]. En general, estos modos de interacción pueden ser de tres tipos: electrostática (binding externo), binding de superficie en los surcos de la doble hélice del ADN e intercalación. La interacción de tipo electrostática está fuertemente determinada por la carga positiva del metal central y la naturaleza de los ligandos que son los que definen la carga neta del complejo. Los complejos del tipo $\mathrm{M}\left(\mathrm{L}_{1}\right)\left(\mathrm{L}_{2}\right)_{2}{ }^{+\mathrm{n}}$ donde $\mathrm{M}=\mathrm{Ru}^{\mathrm{II}}$, Os ${ }^{\mathrm{II}}, \mathrm{Rh}^{\mathrm{III}}$, etc. $\mathrm{L}_{1}=$ diimina bidentada $\mathrm{y} \mathrm{L}_{2}=$ 2,2ºipiridina, 1,10 fenantrolina, etc. y que poseen carga +2 ó +3 son fuertemente atraídos por el esqueleto de fosfatos, cargados negativamente, periféricos de la doble hélice del ácido nucleico. Este tipo de interacción se verifica en varios de estos complejos. Sin embargo, para complejos tricarbonílicos de $\mathrm{Re}^{\mathrm{I}}$, con carga +1 la atracción electrostática es menor y por lo tanto este modo de interacción es menos probable. La unión de superficie ocurre en los surcos mayor y menor de la doble hélice y generalmente está relacionado con interacciones por puente de hidrógeno entre especies que tienen grupos aceptores de enlace de $\mathrm{H}$ ó grupos donores, y los heteroátomos de las bases. Los surcos mayor y menor son formas espaciales que quedan definidas por el ángulo y tipo de torción de la hélice. El tamaño y otras características de los mismos, dependen del tipo de conformación espacial adoptado por el ADN (condicionada, a su vez, por el medio) y de la composición de las bases que lo constituyen $[1,32,35]$. Los complejos metálicos que se unen al ADN por intercalación se 
denominan metalointercaladores. Estos complejos contienen ligandos aromáticos heterocíclicos planos y espacialmente extendidos los cuales pueden insertarse y anclarse en el espacio existente entre los pares de bases nitrogenadas apiladas de la doble hélice. El ligando dppz reúne todas las condiciones necesarias para introducirse entre las bases y, por lo tanto, muchos complejos de metales de transición que poseen este ligando interactúan con el ADN por intercalación $[1,8]$. Este tipo de interacción se caracteriza por presentar en los espectros de absorción, una disminución de la absorbancia y corrimiento hacia el rojo cuando el complejo está unido al ADN. Cabe aclarar que si a $\lambda \geq 320 \mathrm{~nm}$ el ADN no absorbe, los cambios en la absorción están relacionados con los cambios en el microambiente del ligando dppz del complejo. Luego de la intercalación entre las bases nitrogenadas los orbitales $\pi^{*}$ del ligando intercalado podrían acoplarse con los orbitales $\pi$ de los pares de bases disminuyendo la energía de la transición $\pi \rightarrow \pi^{*}$ produciendo el efecto batocrómico observado. Por otro lado, los orbitales $\pi$ acoplados estarían, ahora, parcialmente ocupados por electrones, disminuyendo la probabilidad de transición y, consecuentemente, dando como resultado el hipocromismo observado [36].

Por este motivo, asumiremos que el hipocromismo y el corrimiento batocrómico observados en la figura 2, para el complejo de $\operatorname{Re}^{\mathrm{I}}$ en estudio y el Poly[dAdT $]_{2}$, es indicio inequívoco, no solamente de que efectivamente el complejo interactúa con el polinucleótido, sino también que el modo de esta interacción es por intercalación. 
Figura 2: Espectros de absorción de soluciones del (dppz) $\operatorname{Re}^{\prime}(\mathrm{CO})_{3}\left(4,4^{\prime} \text { bipi }\right)^{+}$en presencia y ausencia del Poly[dAdT $]_{2}$ en buffer TRIS $20 \mathrm{mM}-\mathrm{HCl}, 10 \%$ de $\mathrm{MeOH}, \mathrm{ph}=7 .[\mathrm{Re}]=19,8 \mu \mathrm{M}$

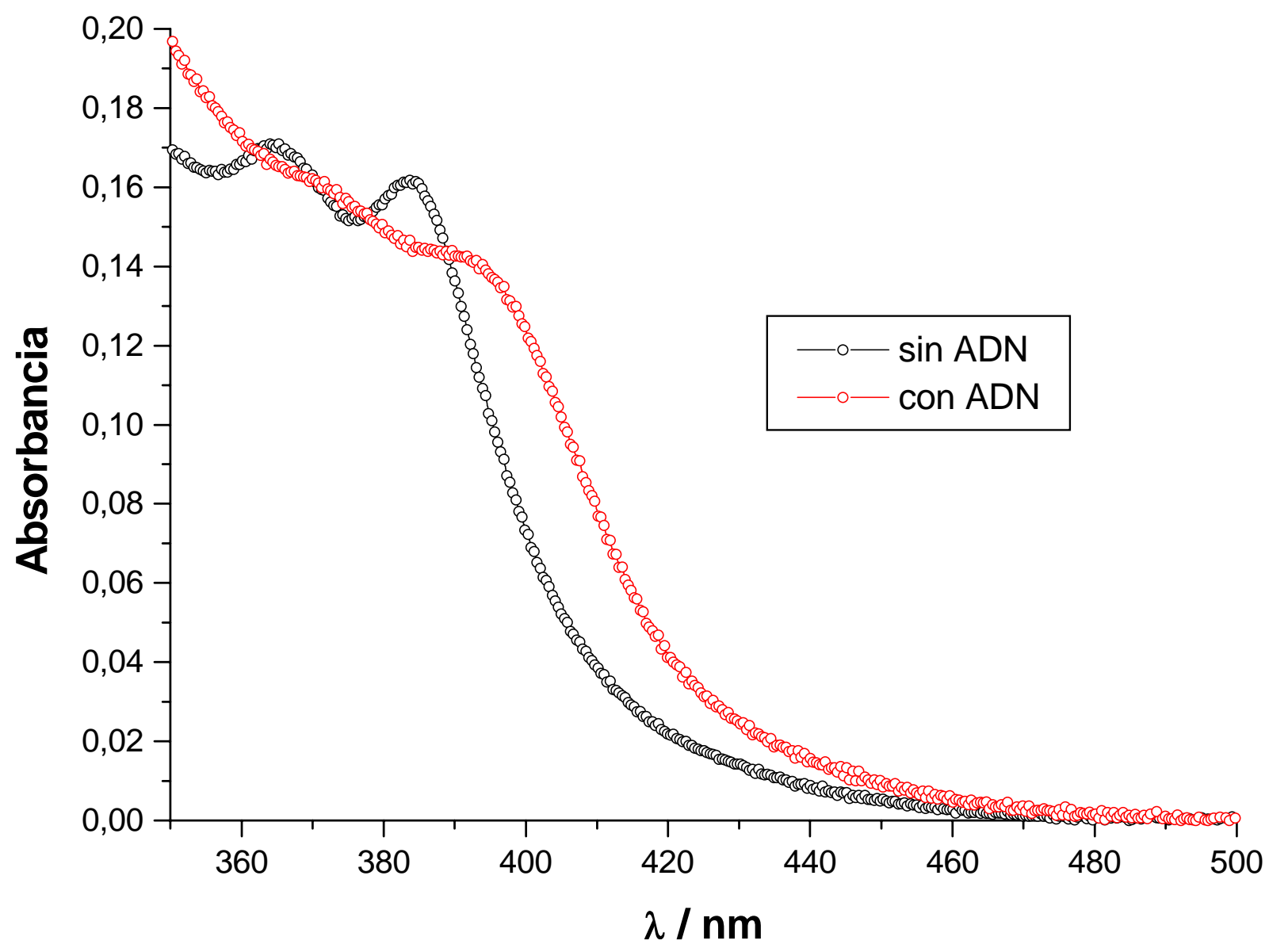

ii. $\quad$ Espectros de emisión

Como vimos en el capítulo anterior, la intensidad de la emisión del complejo $(\mathrm{dppz}) \operatorname{Re}^{\mathrm{I}}(\mathrm{CO})_{3}\left(4,4^{\prime} \text { bipi }\right)^{+}$(solución orgánica) disminuye fuertemente ante el agregado de cantidades creciente de agua. Esto se debe a la desactivación del estado excitado emisor 
producida por las moléculas de $\mathrm{H}_{2} \mathrm{O}$ y por lo tanto, las soluciones del complejo de $\mathrm{Re}^{\mathrm{I}}$ en estudio, en medio acuoso, presentan una luminiscencia muy débil.

Se llevaron a cabo experimentos de emisión en estado estacionario en las soluciones acuosas del complejo (dppz) $\operatorname{Re}^{\mathrm{I}}(\mathrm{CO})_{3}\left(4,4^{\prime} \text { bipi }\right)^{+}$con Poly $[\mathrm{dAdT}]_{2}$ usadas en los experimentos de absorción tomando el espectro de emisión de cada una de ellas. Los espectros de emisión corregidos se obtuvieron entre 450 y $750 \mathrm{~nm}$, excitando con $\lambda_{\text {exc }}=350 \mathrm{~nm}$. Además, las soluciones fueron deaereadas con $\mathrm{N}_{2}$ antes de cada medida. En la figura 3 se muestra una serie de espectros de luminiscencia del complejo de $\operatorname{Re}^{\mathrm{I}}$ en estudio como función de la relación [Poly] / [Re] donde [Re] = 19,8 $\mu \mathrm{M}$ y 0 W[Poly] / [Re] W18,2.

Se observa claramente que la adición de cantidades crecientes de polinucleótido provoca un incremento dramático de la intensidad de emisión del complejo. Esta recuperación de la luminiscencia se detiene, permaneciendo la emisión constante, para soluciones con concentraciones de polinucleótido suficientemente altas (es decir con [Poly] / [Re] $\geq 18,2$ ), lo cual implica que a esa [Poly], ó menor, el $(\mathrm{dppz}) \operatorname{Re}^{\mathrm{I}}(\mathrm{CO})_{3}\left(4,4^{\prime} \text { bipi }\right)^{+}$está completamente unido al Poly[dAdT $]_{2}$.

El efecto de desaparición del quenching por agua y la recuperación de la emisión ha sido ampliamente caracterizado en complejos de $\mathrm{Ru}^{\mathrm{II}}, \mathrm{Os}^{\mathrm{II}}, \mathrm{Rh}^{\mathrm{III}}$, etc., y se lo ha denominado como "molecular ligth-switch" [8]. Aunque existen varios ejemplos en la bibliografía de este fenómeno en complejos de los metales mencionados, solo unos pocos se encuentran descriptos para complejos de $\operatorname{Re}^{\mathrm{I}}[4,5,37]$ de los cuales el último citado corresponde a un complejo que no posee al ligando dppz . 


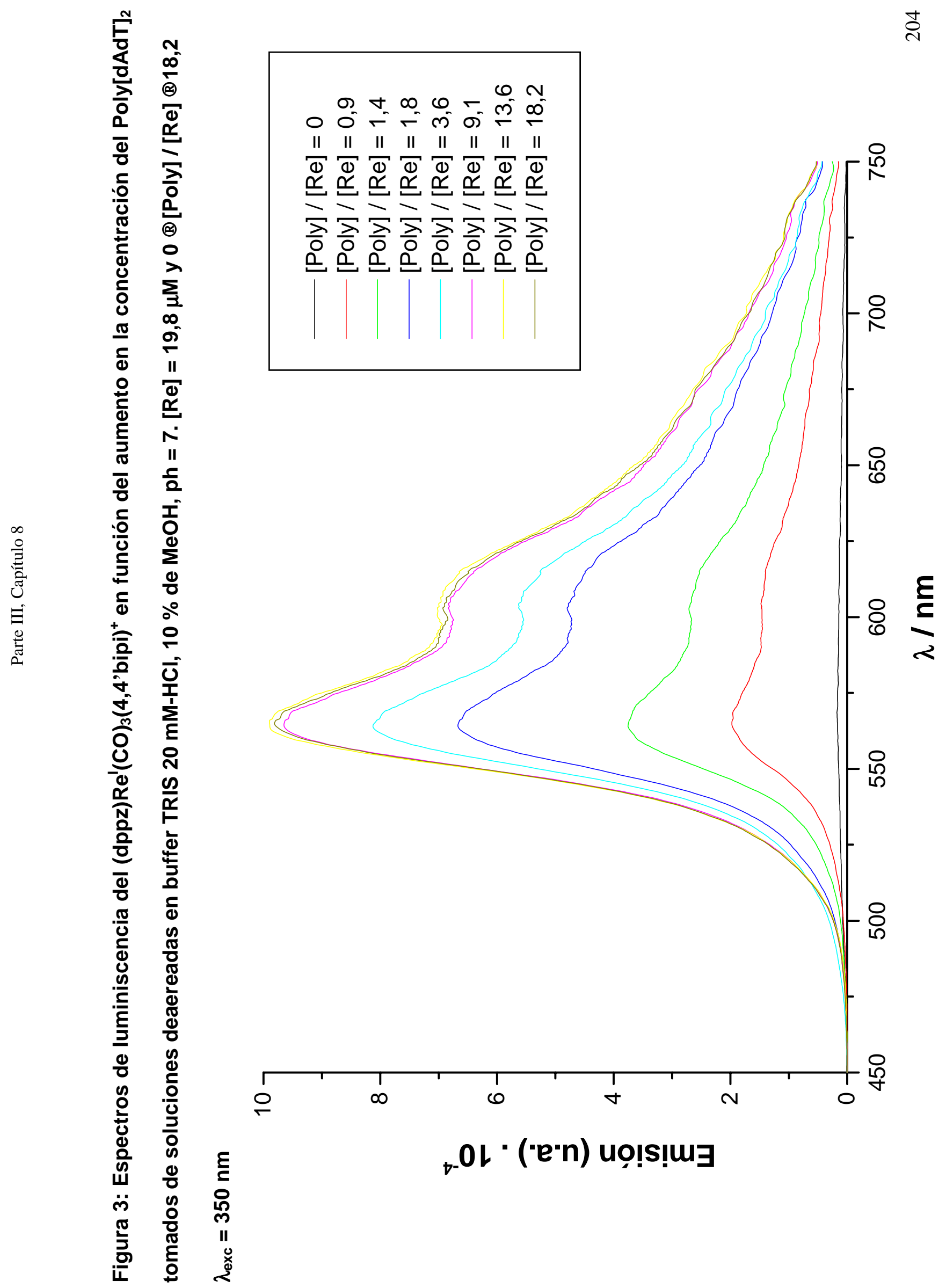


Este efecto se ha determinado que ocurre como resultado de la intercalación del ligando dppz del complejo en la doble hélice del polinucleótido imposibilitando el contacto de éste con las moléculas de $\mathrm{H}_{2} \mathrm{O}$ del solvente $\mathrm{y}$, por consiguiente, evitando la desactivación del estado excitado emisor. Además, el complejo se encontraría ahora en una posición con un entorno hidrofóbico local similar al de un solvente orgánico y por lo tanto, la luminiscencia observada posee los mismos rasgos que la emisión estudiada en estos solventes.

En el caso del (dppz) $\operatorname{Re}^{\mathrm{I}}(\mathrm{CO})_{3}\left(4,4^{\prime} \text { 'bipi }\right)^{+}$la observación del efecto “molecular ligth-switch" aporta mayor evidencia para asumir, en función de lo explicado anteriormente, que el complejo interactúa con el Poly[dAdT $]_{2}$ por intercalación. La luminiscencia observada presenta los mismos rasgos que los observados para la emisión del complejo en acetonitrilo y diclorometano (ver Parte III, capítulo 7). El incremento en la intensidad de la emisión del complejo con el aumento en la relación [Poly] / [Re] pudo ser utilizado para la determinación de la constante de asociación del proceso, como se describe a continuación.

\section{iii. $\quad$ Determinación de la constante de asociación ó constante de binding $K_{b}$}

Los datos obtenidos de los espectros de emisión correspondientes a las soluciones anteriores del complejo de $\operatorname{Re}^{\mathrm{I}}$ en presencia del Poly[dAdT $]_{2}$ fueron empleados para determinar la constante de binding, $\mathrm{K}_{\mathrm{b}}$, utilizando la ecuación de Scatchard modificada por McGhee-von Hippel [38]. El tratamiento de la asociación plantea el establecimiento de un equilibrio entre una especie con un determinado número de sitios de unión para el ligando libre, y el ligando libre. Es decir, en nuestro caso sería:$$
\text { Poly[dAdT }]_{2} \quad+\quad(\text { dppz }) \operatorname{Re}^{\mathrm{I}}(\mathrm{CO})_{3}\left(4,4^{\prime} \text { 'bipi }\right)^{+} \rightleftarrows \text { complejo unido }
$$ 
Además, para obtener la expresión que vincula las concentraciones de cada especie con la $\mathrm{K}_{\mathrm{b}}$ se efectúan consideraciones de tipo estadísticas para describir un tipo de unión aleatoria a uno ó más sitios de unión. El tratamiento utilizado considera también que todos los sitios de unión son equivalentes y que el complejo interactúa de manera no-cooperativa. Para el cálculo de $\mathrm{K}_{\mathrm{b}}$ es necesario determinar, utilizando los datos experimentales, la concentración del complejo libre, $\mathrm{C}_{\mathrm{f}}$, y la concentración del complejo unido $\mathrm{C}_{\mathrm{b}}$, para cada una de las soluciones. Estas concentraciones fueron calculadas utilizando las siguientes ecuaciones [4], que se obtienen asumiendo que la luminiscencia, en todo momento, corresponde a la emisión del complejo libre y unido:

$$
C_{f}=C_{T} \frac{\left[\left(\frac{A}{A_{0}}\right)-P\right]}{(1-P)}
$$

donde $\mathrm{C}_{\mathrm{T}}$ es la suma de las concentraciones del complejo libre y unido, A y Ao son las áreas de los espectros de emisión del complejo en presencia y ausencia de ADN y P es la relación límite de $\mathrm{A} / \mathrm{A}_{0}$, es decir, cuando todo el complejo se encuentra unido al polinucleótido. La expresión de la ecuación de Scatchard modificada por McGhee-von Hippel es la siguiente:

$$
\frac{r}{C_{f}}=K_{b}(1-n r)\left\{\frac{(1-n r)}{[1-(n-1) r]}\right\}^{n-1}
$$

donde $\mathbf{r}=\frac{\mathbf{C}_{\mathbf{b}}}{[\text { Poly }]}, \mathbf{K}_{\mathbf{b}}$ es la constante intrínsica de binding del complejo de $\operatorname{Re}^{\mathrm{I}}$ al ADN y $\boldsymbol{n}$ es el tamaño promedio del sitio de unión en pares de bases. Esto quiere decir que se asume que 
cuando el complejo está unido al ADN cubre (es decir, hace inaccesible para otra molécula de complejo) $n$ sitios de unión consecutivos. Por lo tanto el valor de $n$ nos dice cuantos pares de bases dejan de estar disponibles cuando se produce la interacción de una molécula del complejo. Este tratamiento es de aplicación general y ha sido ampliamente utilizado para sistemas donde el ligando libre no sólo era un complejo de metal de transición [4,39], sino también para estudiar la interacción de proteínas, porfirinas con iones metálicos, diferentes tipos de moléculas orgánicas como drogas, antibióticos, viológenos, etc. [38] con ácidos nucleicos.

En función de esta ecuación se realiza una gráfica de $\mathbf{r} / \mathbf{C}_{\mathbf{f}}$ vs $\mathbf{r}$ obteniéndose los parámetros $\mathrm{K}_{\mathrm{b}} \mathrm{y} n$ por ajuste de los datos experimentales. En las figuras 4 y 5 se muestra el cambio en la relación de áreas de los espectros de emisión con el aumento en la relación [Poly] / [Re] y el mejor ajuste de los datos utilizando los parámetros calculados. El máximo incremento de la emisión se produce para un valor en la relación [Poly] / [Re] $~ 8$ permaneciendo constante luego de la adición de más polinucleótido.

Los valores obtenidos fueron: $\mathrm{K}_{\mathrm{b}}=1,8 \times 10^{5} \mathrm{M}^{-1}$ y $n=2,4$ que están en franca concordancia con los informados en trabajos publicados sobre complejos similares (ver más adelante).

Este valor en la constante de asociación refleja una alta afinidad del complejo por las bases nitrogenadas adenina y timina. El valor de $n$, además, nos está indicando que luego de la unión de una unidad simple de $(\mathrm{dppz}) \operatorname{Re}^{\mathrm{I}}(\mathrm{CO})_{3}\left(4,4^{\prime} \text { bipi }\right)^{+}$son ocupados un poco más de 2 pares de bases del polinucleótido. 
Figura 4: Incremento de la emisión relativa en función del aumento en la concentración del Poly[dAdT $]_{2}$. $A=$ área de emisión en presencia de ADN y $A_{0}=$ área de emisión en ausencia de ADN

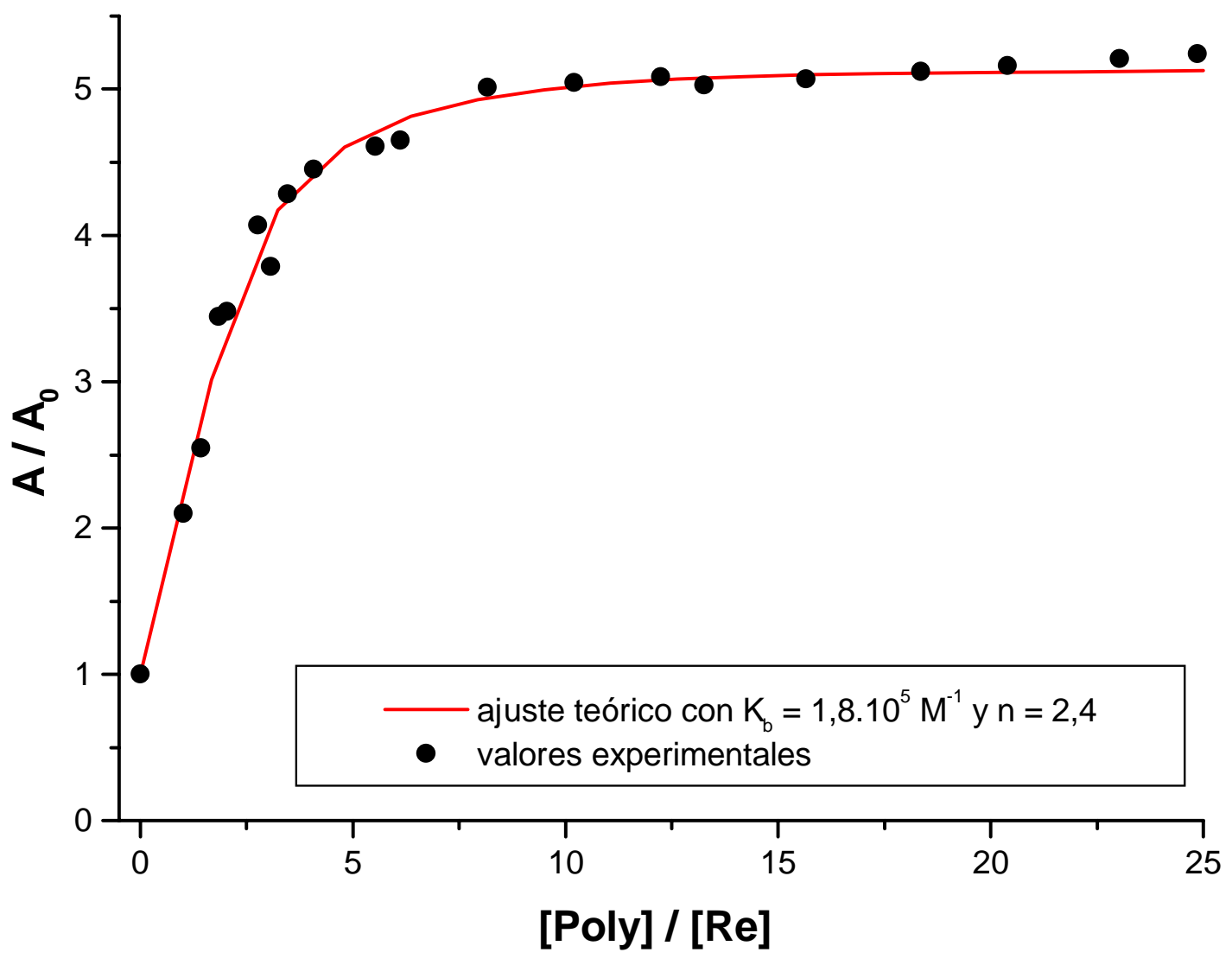


Figura 5: Representación de la ecuación de Scatchard modificada por McGheevon Hippel correspondiente a la interacción entre el $(\mathrm{dppz}) \operatorname{Re}^{\prime}(\mathrm{CO})_{3}\left(4,4^{\prime} \text { bipi }\right)^{+}$y el Poly[dAdT $]_{2}$. Ajuste de los valores experimentales realizado para el cálculo de $K_{b}$ y $n$

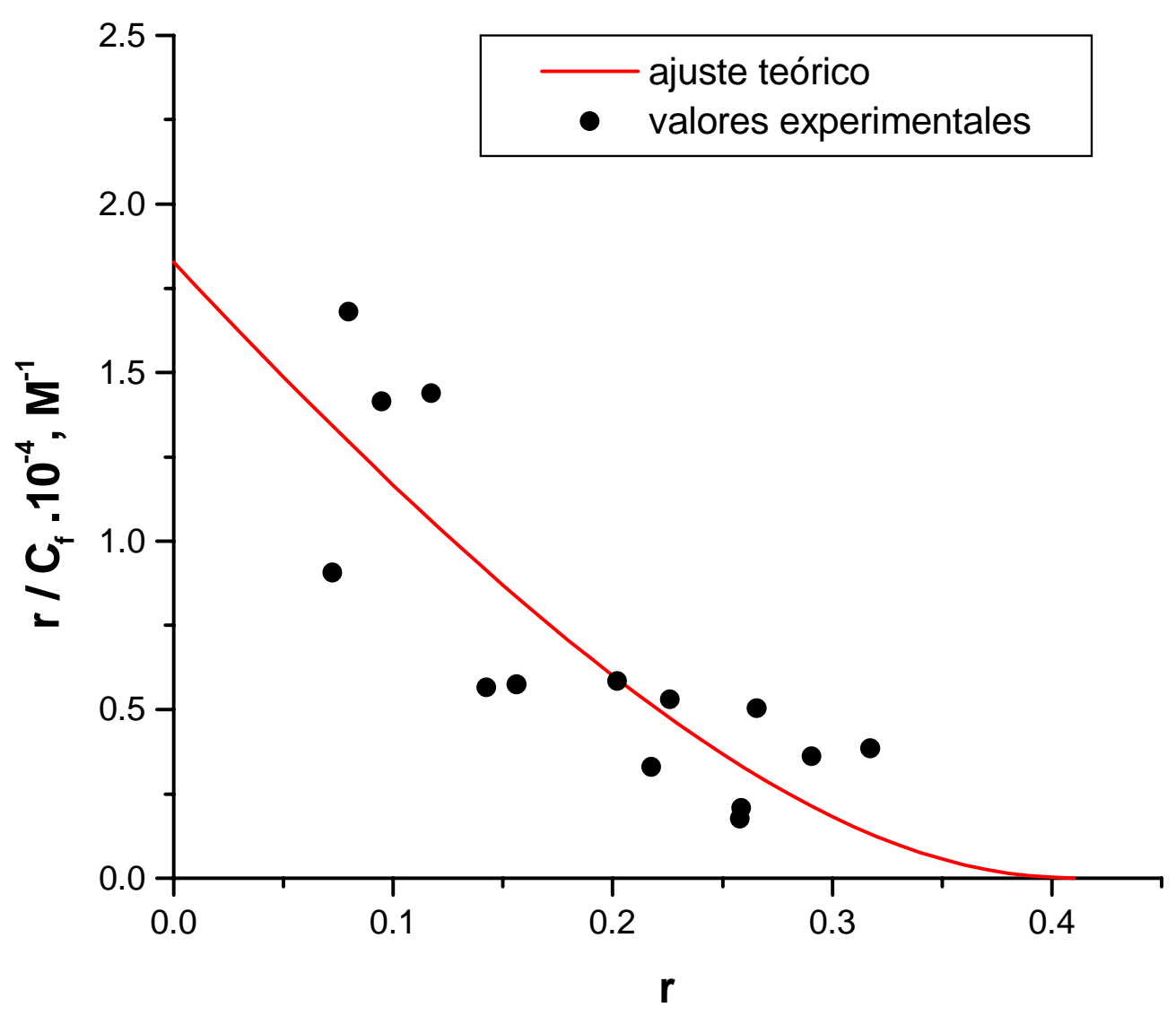




\section{Estudios de interacción realizados entre el $(\mathrm{dppz}) \operatorname{Re}^{\mathrm{I}}(\mathrm{CO})_{3}\left(4,4^{\prime} \text { 'bipi }\right)^{+}$y el Poly $[\mathrm{dGdC}]_{2}$}

\section{i. $\quad$ Espectros de absorción $U V$-visible}

Las soluciones utilizadas se prepararon de manera similar a las descriptas anteriormente para el Poly[dAdT $]_{2}$, es decir, mezclando cantidades crecientes tomadas de una solución madre del polinucleótido Poly $[\mathrm{dGdC}]_{2}$ con un volumen constante de una solución del complejo $(\mathrm{dppz}) \operatorname{Re}^{\mathrm{I}}(\mathrm{CO})_{3}\left(4,4^{\prime} \text { bipi }\right)^{+}$en un volumen final de $1 \mathrm{ml}$ utilizando el buffer TRIS $20 \mathrm{mM}-\mathrm{HCl}$, $10 \%$ de $\mathrm{MeOH}, \mathrm{ph}=7$ como solvente.

Se tomaron los espectros de absorción en presencia y ausencia del Poly[dGdC $]_{2}$ y en ellos se observó, también, el efecto hipocrómico y un suave corrimiento hacia el rojo de las bandas de absorción que se encuentran en la zona comprendida entre 350 y 400 nm, figura 6.

Estas observaciones indican que el complejo de $\operatorname{Re}^{\mathrm{I}}$ en estudio también interactúa con este polinucleótido y sugiere, también, que el modo de unión es por intercalación.

En forma similar a lo ocurrido con el Poly[dAdT $]_{2}$, tampoco se pudo calcular, con estos datos experimentales, una constante de asociación que caracterizara el proceso debido a la alta dispersión encontrada en el análisis de los espectros. 
Figura 6: Espectros de absorción de soluciones del (dppz) $\operatorname{Re}^{\prime}(\mathrm{CO})_{3}\left(4,4^{\prime} \text { bipi }\right)^{+}$en presencia y ausencia del Poly[dGdC] $]_{2}$ en buffer TRIS $20 \mathrm{mM}-\mathrm{HCl}, 10 \%$ de $\mathrm{MeOH}, \mathrm{ph}=7 .[\mathrm{Re}]=19,8 \mu \mathrm{M}$

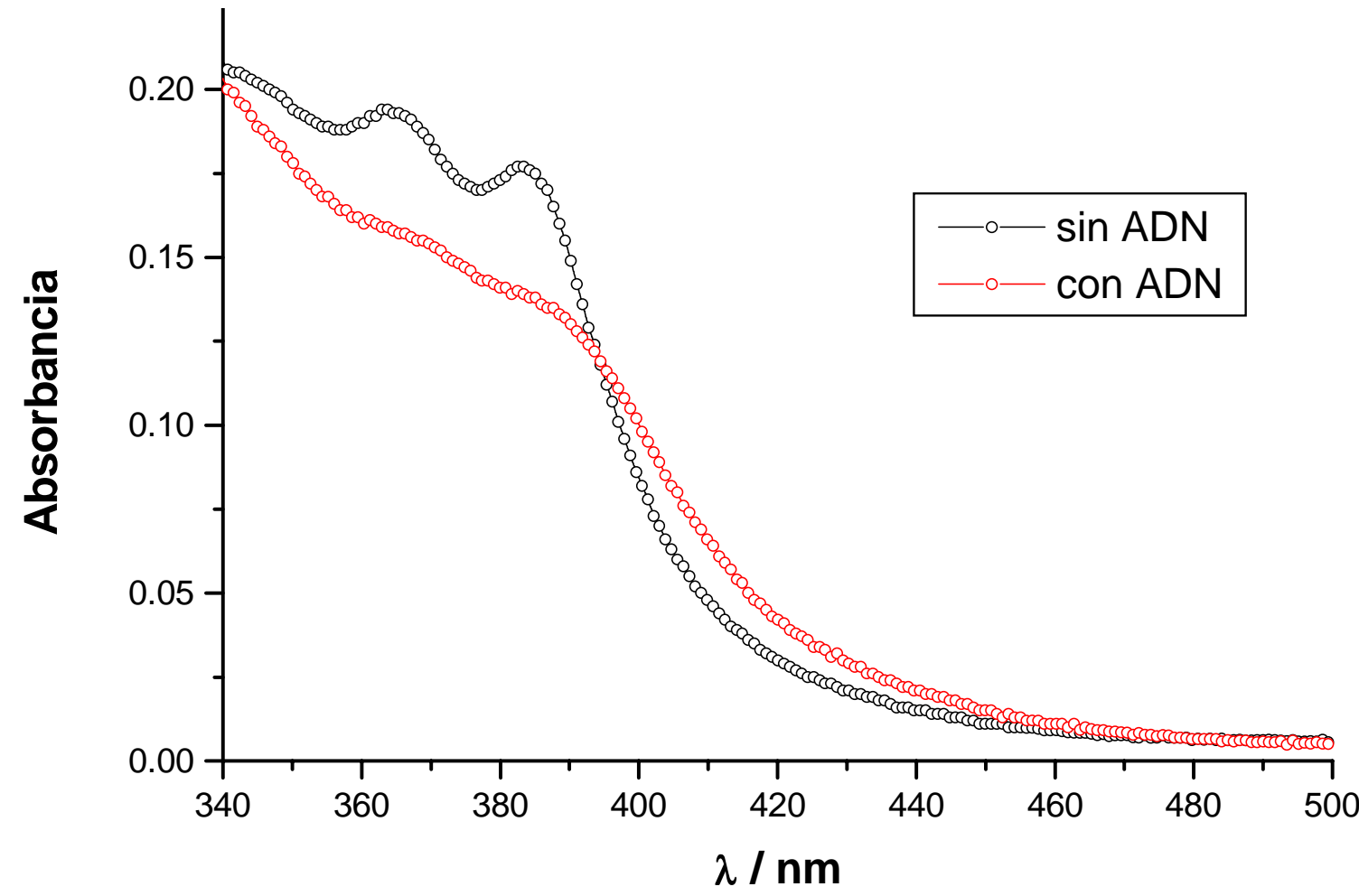

ii. $\quad$ Espectros de emisión

De manera similar a los experimentos realizados con el Poly[dAdT $]_{2}$ se tomaron los espectros de emisión para las soluciones deaereadas del $(\mathrm{dppz}) \mathrm{Re}^{\mathrm{I}}(\mathrm{CO})_{3}\left(4,4^{\prime} \text { bipi }\right)^{+}$que contenían Poly $[\mathrm{dGdC}]_{2}$, figura 7.

A diferencia con lo observado con el Poly[dAdT $]_{2}$, no se observa un incremento importante en la intensidad de emisión en función del aumento en la relación [Poly] / [Re]. Todas las soluciones muestran bajas intensidades de emisión. Esto se refleja en el alto ruido de las curvas, a pesar de ser el resultado del promedio de 3 espectros tomados en forma consecutiva. 
Del análisis de la emisión relativa, A/A 0 , en función de [Poly] / [Re] se puede observar que se produce un pequeño incremento en la intensidad de la emisión a bajas concentraciones del polinucleótido, figura 8. Este aumento de la emisión se verifica cuando 0 [Poly] / [Re] $\square$ 1,6. Posteriormente, al aumentar la relación [Poly] / [Re] se produce una disminución de la emisión hasta alcanzar un valor final constante prácticamente igual al de la solución en ausencia del Poly[dGdC $]_{2}(1,6 \square[$ Poly $] /[\operatorname{Re}] \square 21,8)$.

Figura 7: Espectros de luminiscencia del $(\mathrm{dppz}) \mathrm{Re}^{\prime}(\mathrm{CO})_{3}\left(4,4^{\prime} \text { bipi }\right)^{+}$en función del aumento en la concentración del Poly[dGdC $]_{2}$ tomados de soluciones deaereadas en buffer TRIS $20 \mathrm{mM}-\mathrm{HCl}, 10 \%$ de $\mathrm{MeOH}, \mathrm{ph}=7 ; \lambda_{\text {exc }}=350 \mathrm{~nm}$.

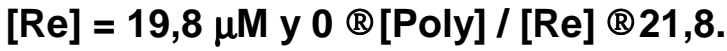

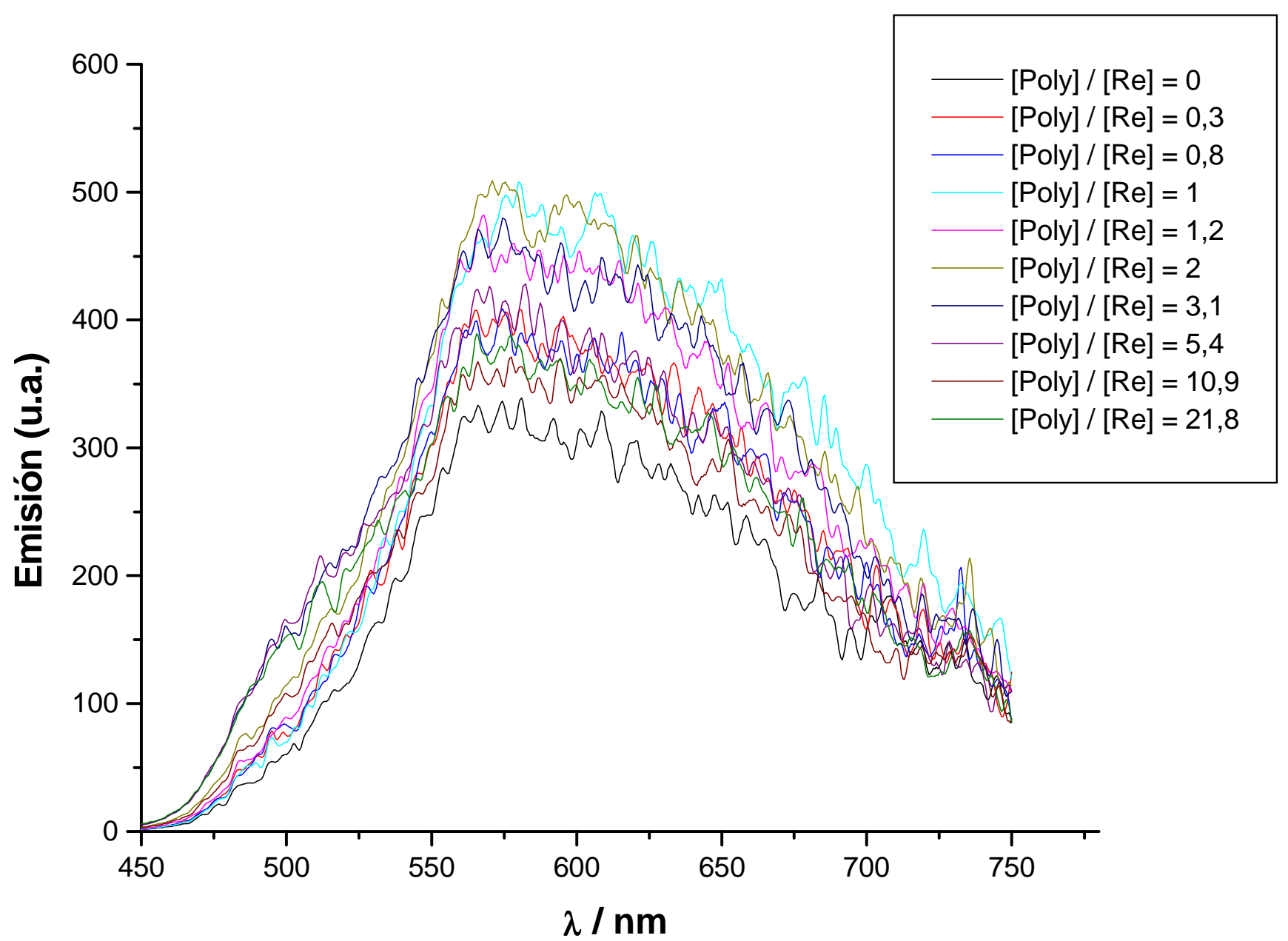


Figura 8: Incremento de la emisión relativa en función del aumento en la concentración del Poly[dGdC $]_{2}$ A = área de emisión en presencia de ADN y $A_{0}=$ área de emisión en ausencia de ADN

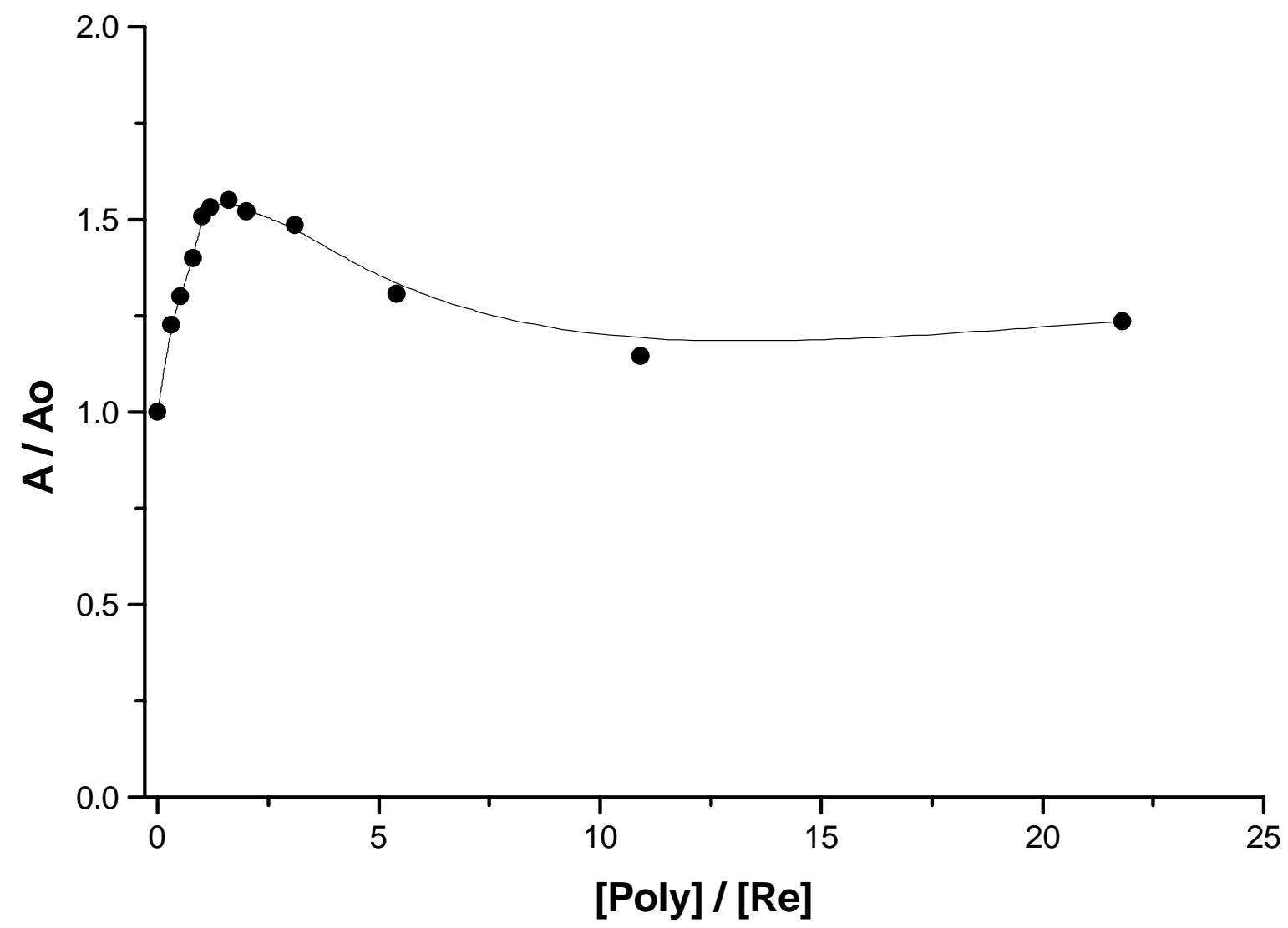

Estos resultados sugieren que, si bien el complejo interactúa con el Poly[dGdC $]_{2}$, probablemente, por intercalación entre las bases guanina y citosina, la afinidad del $(\mathrm{dppz}) \operatorname{Re}^{\mathrm{I}}(\mathrm{CO})_{3}\left(4,4^{\prime} \text { bipi }\right)^{+}$por este polinucleótido sería muy baja. La baja intensidad de emisión y la disminución de la luminiscencia observada a concentraciones mayores de ADN podrían estar relacionados con procesos de quenching que se discutirán más adelante. 


\section{Conclusiones}

Los estudios de interacción en buffer acuoso llevados a cabo utilizando técnicas espectroscópicas entre el complejo $(\mathrm{dppz}) \operatorname{Re}^{\mathrm{I}}(\mathrm{CO})_{3}\left(4,4^{\prime} \text { bipi }\right)^{+}$y los polinucleótidos sintéticos Poly $[\mathrm{dAdT}]_{2}$ y Poly $[\mathrm{dGdC}]_{2}$ sugieren que el complejo de $\mathrm{Re}^{\mathrm{I}}$ interactúa con el polinucleótido por intercalación, entre las bases nitrogenadas, del ligando dppz. Este modo de unión parece ser más efectiva con el Poly $[\mathrm{dAdT}]_{2}$ que con el Poly $[\mathrm{dGdC}]_{2}$. Estos resultados sugieren cierta "preferencia" en la interacción del complejo por las bases A y T. Observaciones similares sobre la mayor afinidad por los pares de bases adenina-timina fueron realizadas anteriormente para el complejo pariente $(\mathrm{dppz}) \operatorname{Re}^{\mathrm{I}}(\mathrm{CO})_{3}(\mathrm{py})^{+}(\mathrm{py}=$ piridina) [4]. En ese caso, la unión preferencial por el polinucleótido Poly $(\mathrm{dA}) \bullet \operatorname{Poly}(\mathrm{dT})$ pudo ser explicada por el hecho de que la posibilidad de torción de la hélice de los pares de bases está relativamente favorecida para la secuencia $\mathrm{dA} \cdot \mathrm{dT}$ y esto podría aliviar efectos estéricos asociados con los ligandos coordinados al metal central que no participan de la intercalación [4]. Similar especificidad por los pares de bases AT también ha sido observada para otros complejos de metales de transición como $\mathrm{Cu}(\mathrm{bcp})_{2}{ }^{+}\left(\mathrm{bcp}=2,9\right.$-dimetil-4,7-difenil-1,10-fenantrolina) y $\mathrm{Ru}(\mathrm{phen})_{3}{ }^{+2}$ (phen $=1,10$-fenantrolina) [40], así como también para otros complejos de $\mathrm{Ru}$ II con ligandos de bipiridinas y fenantrolinas y que poseen el ligando intercalador dppz [8].

El valor de la constante intrínseca de binding, calculada de los experimentos donde se producía un incremento notable en la intensidad de emisión para el Poly[dAdT $]_{2}$, también sugiere una alta afinidad por los pares de base AT. En la tabla I se muestran los valores de $\mathrm{K}_{\mathrm{b}}$

encontrados en la literatura para complejos de $\operatorname{Re}^{\mathrm{I}}$ parientes al complejo $\operatorname{Re}^{\mathrm{I}}$ en estudio y diferentes tipos de ácidos nucleicos: 
Tabla I: Constante intrínsica de binding, $\mathrm{Kb}$, del $(\mathrm{dppz}) \operatorname{Re}^{\prime}(\mathrm{CO})_{3}\left(4,4^{\prime} \mathrm{bipi}^{+}\right.$y de complejos parientes

$\begin{array}{llll}\mathrm{K}_{\mathrm{b}}, \mathrm{M}^{-1} & n & \text { ADN } & \text { Ref. }\end{array}$

\begin{tabular}{|c|c|c|c|c|}
\hline$(\mathrm{dppz}) \operatorname{Re}^{\mathrm{I}}(\mathrm{CO})_{3}\left(4,4^{\prime} \text { bipi }\right)^{+}$ & $1,8.10^{5}$ & 2,4 & Poly $[\mathrm{dAdT}]_{2}$ & este trabajo \\
\hline & --- & --- & Poly $[\mathrm{dGdC}]_{2}$ & este trabajo \\
\hline$(\mathrm{dppz}) \operatorname{Re}^{\mathrm{I}}(\mathrm{CO})_{3}(4-\mathrm{Mepy})^{+\mathrm{a}}$ & $6,0.10^{5}$ & 3,2 & Timo de ternero & [5] \\
\hline$(\mathrm{dppz}) \operatorname{Re}^{\mathrm{I}}(\mathrm{CO})_{3}(\mathrm{py})^{+\mathrm{b}}$ & $4,2.10^{4}$ & 2 & Timo de ternero & [4] \\
\hline$\left(\right.$ benzo-dppz) $\operatorname{Re}^{\mathrm{I}}(\mathrm{CO})_{3}(\mathrm{py})^{+\mathrm{b}}$ & $6,4.10^{4}$ & --- & Timo de ternero & [4] \\
\hline$\left(2,2^{\prime}\right.$ bipi $) \operatorname{Re}^{\mathrm{I}}(\mathrm{CO})_{3}\left(\text { py- } \mathrm{R}_{1}\right)^{+\mathrm{b}, \mathrm{c}}$ & $4,6.10^{5}$ & 2 & Timo de ternero & [37] \\
\hline$\left(2,2^{\prime}\right.$ bipi $) \operatorname{Re}^{\mathrm{I}}(\mathrm{CO})_{3}\left(\text { py- } \mathrm{R}_{2}\right)^{+\mathrm{b}, \mathrm{c}}$ & sin interacción & & Timo de ternero & [37] \\
\hline$(\mathrm{dppz}) \mathrm{Ru}^{\mathrm{II}}\left(2,2^{\prime} \mathrm{bipi}_{2}{ }_{2}{ }^{2}\right.$ & --- & --- & Poly $[\mathrm{dGdC}]_{2}$ & [5] \\
\hline & $4.10^{6}$ & --- & Timo de ternero & \\
\hline
\end{tabular}

a) 4-Mepy = 4-metilpiridina.

b) py $=$ piridina

c) $\mathrm{R}_{1}=-\mathrm{CH}_{2}-\mathrm{NH}-\mathrm{CO}\left(\mathrm{CH}_{2}\right)_{3} \mathrm{NH}-\mathrm{CH}_{2}-(9-\mathrm{An})$ donde 9-An = 9antracenil. $\mathrm{R}_{2}=-\mathrm{CH}_{2}-\mathrm{NH}-\mathrm{CO}-\mathrm{Ph}$ donde $\mathrm{Ph}=$ fenil.

El valor calculado para la $\mathrm{K}_{\mathrm{b}}$ del $(\mathrm{dppz}) \mathrm{Re}^{\mathrm{I}}(\mathrm{CO})_{3}\left(4,4^{\prime} \text { bipi }\right)^{+}$se encuentra en franca concordancia con los informados para complejos parientes de Re ${ }^{I}$. Si bien la intercalación parece ser el mecanismo principal para el binding de este complejo al ADN, podría no ser el 
único. El alto valor de $\mathrm{K}_{\mathrm{b}}$, podría estar relacionado con un efecto de atracción electrostática previo a la intercalación debido a la posible protonación del $\mathrm{N}$ libre del ligando 4,4'bipi en medio acuoso que fortalecería la unión por aumento de la densidad de carga positiva del complejo. No se pueden descartar, además, efectos estéricos adicionales que pueden jugar un rol importante en este tipo de interacción.

El valor de $\mathrm{K}_{\mathrm{b}}$ para los complejos de $\mathrm{Re}^{\mathrm{I}}$, además, es notablemente inferior que la constante de binding del $(\mathrm{dppz}) \mathrm{Ru}^{\mathrm{II}}\left(2,2^{\prime} \text { bipi }\right)_{2}{ }^{+2}$, que es representativa de las obtenidas para la mayoría de los complejos de $\mathrm{Ru}^{\mathrm{II}}\left(\mathrm{K}_{\mathrm{b}}>10^{6}\right)$. Esto se debe, como señalamos anteriormente, fundamentalmente, a que el complejo de $\mathrm{Ru}^{\mathrm{II}}$ es un dicatión mientras que el complejo de $\mathrm{Re}^{\mathrm{I}}$ es un monocatión. Por lo tanto, de la combinación de modos diferentes de interacción se pueden obtener, como resultado, uniones más fuertes.

Los experimentos de emisión en estado estacionario llevados a cabo entre el complejo de $\operatorname{Re}^{\mathrm{I}}$ en estudio y el Poly $[\mathrm{dGdC}]_{2}$ no posibilitaron el cálculo de $\mathrm{K}_{\mathrm{b}}$ en este caso. En efecto, el fuerte incremento de la emisión observada con el Poly[dAdT $]_{2}$ contrasta notablemente con el pequeño, y posterior insignificante, aumento de la luminiscencia observada para el Poly $[\mathrm{dGdC}]_{2}$. El único experimento equivalente encontrado en la bibliografía para un complejo similar correponde a la interacción estudiada entre el complejo (dppz) $\operatorname{Re}^{\mathrm{I}}(\mathrm{CO})_{3}(\mathrm{py})^{+}$ y el polinucleótido $\operatorname{Poly}(\mathrm{dG}) \bullet P o l y(\mathrm{dC})$ en el cual una cadena posee la secuencia GGGGGGG...y la cadena complementaria la secuencia CCCCCCC... [4]. En este caso no se observó un aumento de la emisión sino una disminución gradual de la misma a bajas concentraciones del polinucleótido. Luego, la emisión permaneció constante (y en un valor inferior a la emisión del complejo en ausencia de $\mathrm{ADN}$ ) cuando se adicionó más polinucleótido. Este proceso de quenching de la luminiscencia fue atribuido a la posible fotooxidación de la base nitrogenada guanina por el estado excitado del complejo. Similares observaciones han sido realizadas para el complejo $\mathrm{Ru}(\operatorname{tap})_{3}{ }^{+2}(\operatorname{tap}=1,4,5,8-$ 
tetraazafenantreno) y relacionadas con el poder oxidante del estado excitado del complejo frente a la base más fácilmente oxidable como lo es la guanina [41] en un proceso de transferencia de electrones donor-aceptor. En el $(\mathrm{dppz}) \operatorname{Re}^{\mathrm{I}}(\mathrm{CO})_{3}\left(4,4^{\prime} \text { bipi }\right)^{+}$el proceso de quenching por la guanina también se pone en evidencia. A bajas concentraciones del Poly $[\mathrm{dGdC}]_{2}$, el efecto protector sobre el quenching de la emisión producido por el agua, producto de la intercalación, parece ser suficiente para compensar levemente la pérdida de la intensidad de la luminiscencia debido al quenching de la guanina dando como resultado un incremento leve de la emisión. Posteriormente, al aumentar la cantidad de polinucleótido, un incremento en el número de moléculas de complejo unidas podría favorecer la orientación entre el complejo excitado aceptor y la guanina donor para un proceso de quenching óptimo, lo que justificaría la disminución de la emisión observada para [Poly] / [Re] > 1,6.

Los resultados de este trabajo sugieren que el complejo $(\mathrm{dppz}) \operatorname{Re}^{\mathrm{I}}(\mathrm{CO})_{3}\left(4,4^{\prime} \text { bipi }\right)^{+}$podría presentar cierta selectividad de base al interactuar con ADN en cuya composición estén presentes las cuatro bases nitrogenadas, A, T, G y C, privilegiando la unión en secuencias ricas en pares de bases AT. Esta interesante propiedad permitiría la posibilidad de desarrollar sondas fluorescentes para la identificación de determinadas secuencias de base para biomoléculas tan importantes como los ácidos nucleicos. Esto ha sido sugerido para complejos similares que poseen el ligando dppz y en la actualidad ya se ha mostrado cierto grado de avance en ese sentido, siendo un área de investigación a abordar en el futuro $[1,8,31,35]$. 
Bibliografía consultada para la Parte III del presente trabajo de tesis

[1] A. Kirsch-De Mesmaeker, J-P Lecomte and J.M. Kelly, Topics en Current Chemistry 1996, 177, 25-76.

[2] K:S. Shanze, D.B. MacQueen, T.A. Perkins and L.A. Cabana, Coord. Chem. Rev., 1993, $122,63$.

[3] a) Wrighton M and Morse D.L., J. Am. Chem. Soc., 96 (1974) 998. b) P.J. Giordano and M.S. Wrighton, J. Am. Chem. Soc., 101 (1979) 2888. c) Worl L.A., Duesing R., Chen P., Della Ciana L. and Meyer T.J., J. Chem. Soc., Dalton Trans., 1991, 849. d) Striplin D.R. and Crosby G.A., Chem. Phys. Lett., 1994, 221, 426.

[4] a) V.W. Yam, K.K Lo, K. Cheung and R.Y. Kong, J. Chem. Soc., Chem. Commun., 1995, 1191. b) V.W. Yam, K.K Lo, K. Cheung and R.Y. Kong, J. Chem. Soc., Dalton Trans., 1997, 2067-2072

[5] H.D. Stoeffler, N.B. Thornton, S.L. Temkin and K. Schanze, J. Am. Chem. Soc. 1995, $117,7119-7128$

[6] W.D. Bates, P. Chen, D.M. Dattelbaum, W.E. Jones Jr., and T.J. Meyer. J. Phys.Chem. A $1999,103,5227-5231$

[7] M.R. Waterland, K.C. Gordon, J.J. Mcgarvey and P.M. Jayaweera, J. Chem. Soc., Dalton Trans., 1998, 609-616

[8] K.E. Ekkila, D.T. Odom and J.K. Barton, Chem. Rev. 1999, 99, 2777-2795.

[9] C Hiort, P. Lincoln and B. Nordén. J. Am. Chem. Soc. 1993, 115, 3448-3454.

[10] Dickeson, J. E; Summers, L.A. Aust. J. Chem. 1970, 23, 1023.

[11] Yamada, Masaki; Tanaka, Yoshihito; Yoshimoto, Yasuyuki; Kuroda, Shigeyasu and Shimao, Ichiro. Bull. Chem. Soc. Jpn. 1992, 65 (4), 1006-11.

[12] J.V. Caspar and T.J. Meyer, J. Phys. Chem., 1983, 87, 952 
[13] R.D. Gillard, R.E.E. Hill and R. Maskill, J. Chem. Soc. A, 1970, 1447.

[14] E. Amouyal, A. Homsi, J.-C. Chambron and J.-P Sauvage, J. Chem. Soc., Dalton Trans., 1990, 1841.

[15] Bates, W.D. Ph.D. Dissertation, University of North Carolina at Chapel Hill, Chapel Hill, NC, 1995.

[16] M. R. Féliz, F. Rodriguez Nieto, G. Ruiz and E. Wolcan, J. Photochem. Photobiol. A: Chem 117 (1998) 185-192.

[17] M.N. Ackermann and L.V. Interrante, Inorg. Chem., 1984, 23, 3904.

[18] J. Fees, M. Ketterle, A. Klein, J. Fiedler and W. Kaim. J. Chem. Soc., Dalton Trans., $1999,2595-2599$

[19] J. Fees, W. Kaim, M. Moscherosch, W. Matheis, J. Klíma, M Krejpík and S. Záliš. Inorg. Chem., 1993, 32, 166-174.

[20] A. Lewanowicz and J. Lipinski. J. Mol. Struct., 450 (1998) 163-169

[21] Joseph R. Lakowicz “Principles of Fluorescence Spectroscopy”, Plenum Press Ed., New York 1983. Turro, N. "Modern Molecular Photochemistry", Ed. University Science Books, 1991.

[22] I. Carmichael and G.L. Hug. J. Phys. Chem. Ref. Data, Vol. 15, No. 1, 1986

[23] J.R. Schoonover, G.F. Strouse, R.B. Dyer, W.D. Bates, P. Chen, and Thomas J.Meyer. Inorg. Chem., 1996, 35, 273-274

[24] C. Turro, S.H. Bossman, Y. Jenkins, J.K. Barton and N.J. Turro, J. Am. Chem. Soc. $1995,117,9026$.

[25] R.B. Nair, B.M. Cullum and C.J. Murphy, Inorg. Chem., 1997, 36, 962-965.

[26] Kosower E:M. and Cotter J.C. J. Am. Chem. Soc. 1964, 86, 5525. V. Ramamurthy and K.S. Schanze, "Molecular and Supramolecular Photochemistry. Vol. 2: Organic and Inorganic Photochemistry ” Marcel Dekker, Inc., New York 1998. 
[27] C.J. Kleverlaan and D.J. Stufkens, Inorg. Chim. Acta, 1999, 284, 61.

[28] M. Féliz, G. Ferraudi, H. Altmiller, J.Phys.Chem., 1992, 96, 257. G. Ruiz, E. Wolcan and M. R. Féliz, J. Photochem. Photobiol. A:Chem. 101 (1996) 119-125

[29] Shaw, J.R. and Schmehl, R.H. J. Am. Chem. Soc. 1991, 113, 389.

[30] Shaw, J.R., Webb, R.T. and Schmehl, R.H. J. Am. Chem. Soc. 1990, 112, 1117.

[31] J.N. Demas and B.A. DeGraff, “Applications of Inorganic Photochemistry”, J. Chem. Educ. 1997, Vol 74, Nro.6, 690-695. G. Orellana, Conferencia Plenaria, “VII ELAFOT”, Viña del Mar, Chile, 2002.

[32] L. Stryer, “Biochemistry”, Freeman, Ney York, 1988.

[33] Wells, R.D., et al., J. Mol. Biol. 54, 465 (1970).

[34] R.E. Holmlin, E.D.A. Stemp and J.K. Barton, Inorg. Chem., 1998, 37, 29-34

[35] P.J. Carter, S.A. Ciftan, M.F. Sistare and H. Holden Thorp, “Applications of Inorganic Photochemistry”, J. Chem. Educ. 1997, Vol 74, Nro.6, 641-645 .

[36] Q-L. Zhang, J-H Liu, X-Z Ren, H. Xu, Y. Huang, J-Z Liu and L-N Ji, J. Inorg. Biochem. 95, 2003, 194-198.A.M. Pyle, J.P. Rehmann, R. Meshoyrer, C.V. Kumar, N.J. Turro and J.K. Barton, J. Am. Chem. Soc. 111, 1989, 3053.

[37] a) N.B. Thornton and K. Schanze, New J. Chem., 1996, 20, 791-800. b) N.B. Thornton and K. Schanze, Inorg. Chem., 1993, 32, 4994-4995

[38] J.D. McGhee y P.H. von Hippel, J. Mol. Biol. 1974, 86, 469-489

[39] F. Pierard, A. Del Guerzo, A. Kirsch-De Mesmaeker, M. Demeunynck and J. Lhomme, Phys. Chem. Chem. Phys., 2001, 3, 2911-2920.

[40] R. Tamilarasan, S. Ropartz and D.R. McMillin, Inorg. Chem., 1990, 29, 2798. A.B. Tossi and J.M. Kelly, Photochem. Photbiol., 1989, 49, 545.

[41] C. Moucheron, A. Kirsch-De Mesmaeker and J.M. Kelly, J. Photochem. Photobiol. B, 1997, 40, 91-106. 